July 2001 • NREL/SR-500-29950

\title{
WindPACT Turbine Design Scaling Studies: Technical Area 4- Balance-of-Station Cost
}

\section{March 2000-15 March 2001}

D.A. Shafer, K.R. Strawmyer, R.M. Conley, J.H. Guidinger, D.C. Wilkie, and T.F. Zellman With assistance from D.W. Bernadett

Commonwealth Associates, Inc. Jackson, Michigan

\section{NPE1}

National Renewable Energy Laboratory

1617 Cole Boulevard

Golden, Colorado 80401-3393

NREL is a U.S. Department of Energy Laboratory

Operated by Midwest Research Institute $\bullet$ Battelle $\bullet$ Bechtel

Contract No. DE-AC36-99-G010337 
July 2001 - NREL/SR-500-29950

\section{WindPACT Turbine Desing Scaling Studies: Technical Area 4- Balance-of-Station Cost}

\section{March 2000—15 March 2001}

D.A. Shafer, K.R. Strawmyer, R.M. Conley, J.H. Guidinger, D.C. Wilkie, and T.F. Zellman With assistance from D.W. Bernadett

Commonwealth Associates, Inc. Jackson, Michigan

NREL Technical Monitor: Alan Laxson

Prepared under Subcontract No. YAM-10-30203-02

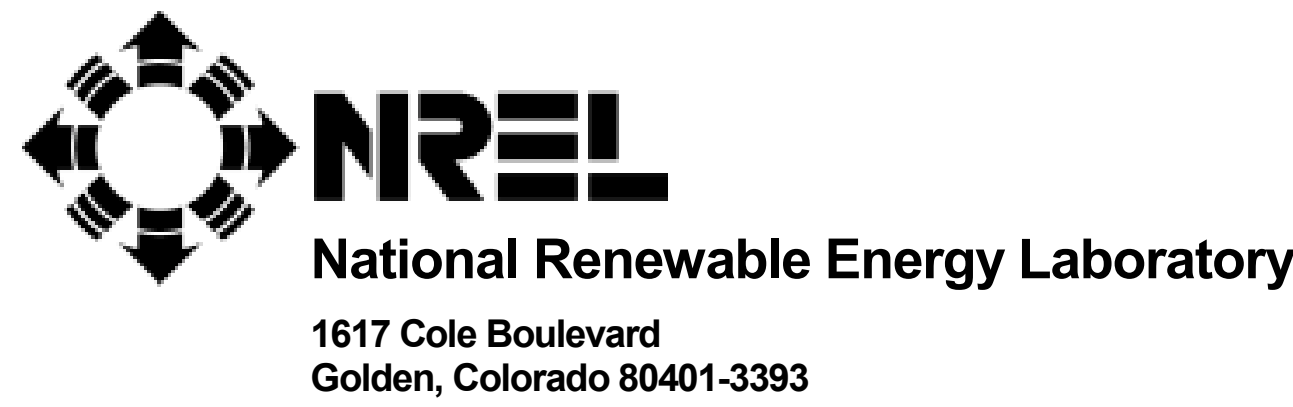

NREL is a U.S. Department of Energy Laboratory Operated by Midwest Research Institute $\bullet$ Battelle $\bullet$ Bechtel Contract No. DE-AC36-99-G010337 


\section{NOTICE}

This report was prepared as an account of work sponsored by an agency of the United States government. Neither the United States government nor any agency thereof, nor any of their employees, makes any warranty, express or implied, or assumes any legal liability or responsibility for the accuracy, completeness, or usefulness of any information, apparatus, product, or process disclosed, or represents that its use would not infringe privately owned rights. Reference herein to any specific commercial product, process, or service by trade name, trademark, manufacturer, or otherwise does not necessarily constitute or imply its endorsement, recommendation, or favoring by the United States government or any agency thereof. The views and opinions of authors expressed herein do not necessarily state or reflect those of the United States government or any agency thereof.

Available electronically at http://www.doe.gov/bridge

Available for a processing fee to U.S. Department of Energy

and its contractors, in paper, from:

U.S. Department of Energy

Office of Scientific and Technical Information

P.O. Box 62

Oak Ridge, TN 37831-0062

phone: 865.576.8401

fax: 865.576.5728

email: reports@adonis.osti.gov

Available for sale to the public, in paper, from:

U.S. Department of Commerce

National Technical Information Service

5285 Port Royal Road

Springfield, VA 22161

phone: 800.553.6847

fax: 703.605.6900

email: orders@ntis.fedworld.gov

online ordering: http://www.ntis.gov/ordering.htm

Printed on paper containing at least $50 \%$ wastepaper, including $20 \%$ postconsumer waste 


\section{TABLE OF CONTENTS}

Executive Summary 1

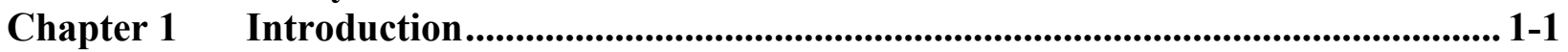

Background ..................................................................................1 1-1

Scope of Technical Area 4 - Balance-of-Station Cost......................... 1-1

Physical Layout of Wind Farm............................................................ 1-2

Utility Power Grid............................................................................................ 1-2

Chapter 2 Conclusions and Recommendations.......................................................... 2-1

Chapter 3 Electrical System ............................................................................................... 3-1

Cost Summary and Conclusions................................................................................ 3-1

Design Guidelines and Assumptions ......................................................... 3-2

Electrical Systems Analysis ........................................................................ 3-4

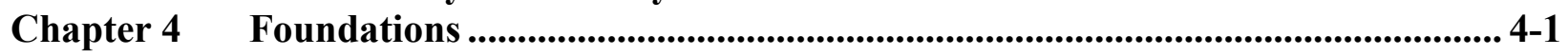

Chapter 5 Other Civil Infrastructure ....................................................................... 5-1

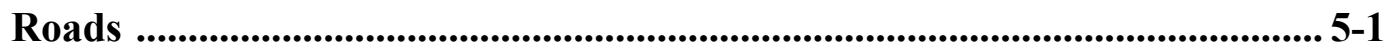

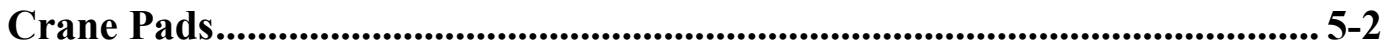

Chapter 6 Miscellaneous Infrastructure................................................................. 6-1

Cost Summary and Conclusions.................................................................... 6-1

Communications System ............................................................................................. 6-2

Tower Lighting and Meteorological Towers .............................................. 6-2

Maintenance Building............................................................................................ 6-3

Chapter 7 Questions and Answers ............................................................................. 7-1

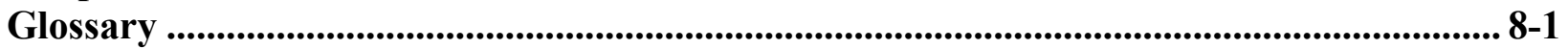

Figures

$\begin{array}{ll}\text { Figure 1 } & \text { Great Plains Region } \\ \text { Figure 2 } & \text { 10-m Wind Rose Graph } \\ \text { Figure 3 } & \text { 750-kW 15-kV Plan } \\ \text { Figure 4 } & \text { 750-kW 25-kV Plan } \\ \text { Figure 5 } & \text { 2.5-MW 25-kV Plan } \\ \text { Figure 6 } & \text { 2.5-MW 35-kV Plan } \\ \text { Figure 7 } & \text { 5-MW 25/35-kV Plan } \\ \text { Figure 8 } & \text { 10-MW 25/35-kV Plan } \\ \text { Figure 9 } & \text { Collector System One-Line Diagram } \\ \text { Figure 10 } & \text { Communications System One-Line Diagram } \\ \text { Figure 11 } & \text { Relative Foundation Geometry } \\ \text { Figure 12 } & \text { Windmill Size and Foundation Comparison }\end{array}$


Figure 13 Foundation Cost

Figure 14 Rock Foundation

Figure 15 Cost Comparison: Pad and Pier vs. Rock Anchor

Appendices

Appendix A Engineering Assumptions and Data ..................................................... A-1

Appendix B System Modeling Data ..........................................................................................B-1

Appendix C Power Flow Study Results......................................................................... C-1

Appendix D Short-Circuit Study Results ................................................................ D-1

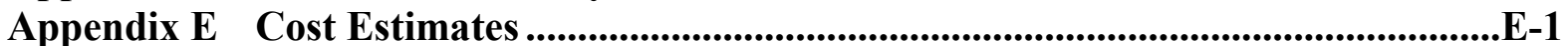




\section{EXECUTIVE SUMMARY}

\section{INTRODUCTION}

The United States Department of Energy has implemented the Wind Partnerships for Advanced Component Technologies (WindPact) program to explore the most advanced wind-generating technologies for improving reliability and decreasing energy costs. The first step in the WindPact program is a scaling study to bound the optimum sizes for wind turbines, to define size limits for certain technologies, and to scale new technologies. The technical activities are divided into the following four projects:

Technical Area 1 - Composite Blades for 80-120-meter Rotors

Technical Area 2 - Turbine, Rotor, and Blade Logistics

Technical Area 3 - Self-Erecting Tower and Nacelle Feasibility

Technical Area 4 - Balance-of-Station Cost

This report covers Technical Area 4 - Balance-of-Station Cost, which includes the electrical power collector system, wind turbine foundations, communications and controls, meteorological equipment, access roadways, crane pads, and the maintenance building. Technical Areas 2 and 4 are both based on a conceptual 50-megawatt (MW) wind farm site near Mission, South Dakota. Cost comparisons are provided for four sizes of wind turbines: 750 kilowatt $(\mathrm{kW}), 2.5 \mathrm{MW}, 5.0$ $\mathrm{MW}$, and 10.0 MW.

\section{SUMMARY OF COSTS}

The table below summarizes the balance-of-station costs (in millions of 2000 dollars) for the smallest and largest sizes studied. The $750-\mathrm{kW}$ turbine requires 66 units to provide $50 \mathrm{MW}$ compared with the 10.0-MW size, which requires only five units.

\begin{tabular}{|c|c|c|c|c|}
\hline \multirow[b]{2}{*}{ Category } & \multicolumn{2}{|c|}{$66-750 \mathrm{~kW}$} & \multicolumn{2}{|c|}{$5-10 \mathrm{MW}$} \\
\hline & $\$ \times 10^{6}$ & Percent & $\$ \times 10^{6}$ & Percent \\
\hline Foundations & 5.2 & 26 & 8.0 & 42 \\
\hline Service Roads & 2.3 & 12 & .7 & 4 \\
\hline Crane Pads & 1.4 & 7 & 1.6 & 8 \\
\hline Total Civil & 8.8 & 45 & 10.3 & 54 \\
\hline Total Electric System & 4.5 & 23 & 3.7 & 19 \\
\hline Total Misc. Infrastructure & 1.7 & 9 & 0.6 & 3 \\
\hline Land, Overheads, and Contingency & 4.6 & 23 & 4.4 & 23 \\
\hline Total Balance of Plant (\$ millions) & 19.7 & 100 & 18.9 & 100 \\
\hline
\end{tabular}

\section{CONCLUSIONS}

1. The largest component of the balance-of-station cost is the civil infrastructure $(45 \%-$ $54 \%$ ), which comprises foundations, service roads, and crane pads. The largest-cost item 
is the foundations, and the second is crane pads. Both of these items show increasing costs for the larger turbine sizes. This is one area that can benefit from additional research. To achieve the lowest total cost, we recommend that future studies evaluate the combined tower and foundation designs. Another area that could also benefit from additional research is eliminating or minimizing crane pads (e.g., by using self-erecting towers, Technical Area 3).

2. The electrical system comprises $19 \%-23 \%$ of the balance-of-station costs. The electrical system can be built using conventional utility-grade equipment for the turbine ranges being studied. No new technology or scaling of existing technology is required. The larger units provide some economy of scale with regard to the electrical system.

3. Communications, tower lighting, meteorological towers, and the maintenance building are lumped together under miscellaneous infrastructure in the above table. These items are not a large part of the balance-of-station costs and do demonstrate decreasing costs for the larger units. The decreasing cost results from fewer units, thereby reducing tower lighting costs and communications costs. Existing technology is satisfactory for these needs, and no additional research is needed in these categories.

4. Land, overhead, and contingency are lumped together in the final category above. Because this is a conceptual study, we used a contingency of $20 \%$, which is the largest component of this category. The overhead costs are for balance-of-plant items only and include engineering, surveying, licensing, land acquisition, construction inspection, and the owner's project management. This category does not include land or land acquisition costs for leasing the land for the wind farm itself. It only includes the incremental land costs for transmission lines away from the wind farm and the purchase of property for the maintenance building and substation. This last category is added to provide a total balance-of-plant cost. 


\section{CHAPTER 1 INTRODUCTION}

\section{BACKGROUND}

As wind energy has grown more and more competitive with other forms of energy generation, the margin of improvement from one generation of technology to another has become smaller and smaller. And yet, to achieve the decrease in cost of energy necessary to place wind energy on an equal playing field with fossil-fuel-generated electricity such as natural gas, a reduction in cost of energy of up to $30 \%$ is still required. Wind turbine manufacturers have achieved great results by improving manufacturing techniques, taking advantage of the latest in engineering tools, and applying new concepts to existing designs. However, to achieve the required $30 \%$ reduction, a much greater level of innovation is required.

The United States Department of Energy (DOE) has implemented the Wind Partnerships for Advanced Component Technologies (WindPACT) program to explore the most advanced windgenerating technologies for improving reliability and decreasing the cost of energy. This work is being carried out through the Midwest Research Institute and the National Renewable Energy Laboratory (NREL) in Golden, Colorado.

One of the first steps in the WindPACT program is a set of preliminary Scaling Studies to bound optimum sizes for turbines of the future, to help define sizing limits for certain critical technologies, and to explore the scale possibilities for new advanced concepts. The technical activities for the scaling study are divided into the following four projects:

Technical Area 1 - Composite Blades for 80- to 120-meter Rotors

Technical Area 2 - Turbine, Rotor, and Blade Logistics

Technical Area 3 - Self-Erecting Tower and Nacelle Feasibility

Technical Area 4 - Balance-of-Station Cost

\section{SCOPE OF TECHNICAL AREA 4-BALANCE-OF-STATION COST}

The subject of this report is Technical Area 4-Balance-of-Station Cost, which includes the electrical-power-collector system, wind turbine foundations, communications and controls, meteorological equipment, access roadways, crane pads, and maintenance offices.

Technical Areas 2 and 4 are both based on a conceptual 50-megawatt (MW) wind farm site near Mission, South Dakota (Figure 1). Cost comparisons are provided for four sizes of wind turbines: 750 kilowatt $(\mathrm{kW}), 2.5 \mathrm{MW}, 5 \mathrm{MW}$, and $10 \mathrm{MW}$.

We prepared a draft report in June 2000. NREL held a workshop on November 2 and 3, 2000, to facilitate exchange of information among the various contractors and other interested parties. This final report incorporates comments received at this workshop. 


\section{PHYSICAL LAYOUT OF WIND FARM}

We developed conceptual wind farm layouts for the four turbine sizes (Figures 3 through 8). The 50-MW wind farm requires 66 of the $750-\mathrm{kW}$-size turbines, 20 of the $2.5-\mathrm{MW}$-size turbines, 10 of the 5 -MW-size turbines, or 5 of the 10-MW-size turbines. We arbitrarily numbered each turbine location for identification purposes.

Wind roses from Pierre, South Dakota, and Valentine, Nebraska (Figure 2), show a strong predominance of energy from the northwest and north-northwest. Therefore, the turbines were oriented perpendicular to an assumed prevailing wind direction of $343^{\circ}$ to best use the natural landforms in the study area.

As shown on Figures 3 through 8, the turbines are arranged in a rectilinear array. This approach has been taken for three reasons. First, the gentleness of the terrain allows for array layouts to be constructed without regard for topography. Second, the general scaling nature of the WindPACT study makes rectilinear layouts desirable to facilitate linear interpolation of results to intermediate turbine sizes. Third, site-specific layout optimization is beyond the scope of this project. However, we did some site-specific tailoring of the rectilinear layouts to make them more realistic.

Taking into account the distribution of wind energy, the spacing for lowest cost of energy was determined to be 2.3 rotor diameters between turbines and 12 rotor diameters between rows. We summarized the dimensions for the wind farm layouts below.

Plant Size
$\mathbf{k W}$
750
2,500
5,000
10,000

$\begin{gathered}\text { Rotor } \\ \text { Diameter }\end{gathered}$
$50 \mathrm{~m}(164 \mathrm{ft})$
$85 \mathrm{~m}(279 \mathrm{ft})$
$120 \mathrm{~m}(394 \mathrm{ft})$
$170 \mathrm{~m}(558 \mathrm{ft})$

Minimum Distance
Between Turbines
$115 \mathrm{~m}(378 \mathrm{ft})$
$196 \mathrm{~m}(642 \mathrm{ft})$
$276 \mathrm{~m}(906 \mathrm{ft})$
$391 \mathrm{~m}(1,283 \mathrm{ft})$
Minimum Distance Between Rows $600 \mathrm{~m}(1,969 \mathrm{ft})$ $1,020 \mathrm{~m}(3,347 \mathrm{ft})$ $1,440 \mathrm{~m}(4,725 \mathrm{ft})$ $2,040 \mathrm{~m}(6,693 \mathrm{ft})$

\section{UTILITY POWER GRID}

The local transmission company is the Western Area Power Administration (WAPA). A 115kilovolt $(\mathrm{kV})$ transmission line is within one mile of the area of the proposed wind farm. We developed the technical requirements and costs for the electrical-collector system to interconnect the turbines and to connect them via a new substation to this $115-\mathrm{kV}$ line. There is an existing substation on the line, which serves the town of Mission. However, for this study, we assumed that a new substation would be constructed as the interconnection point. 


\section{CHAPTER 2 CONCLUSIONS AND RECOMMENDATIONS}

\section{$\underline{\text { Balance-of-Station Cost }(\$ \times 1000)}$}

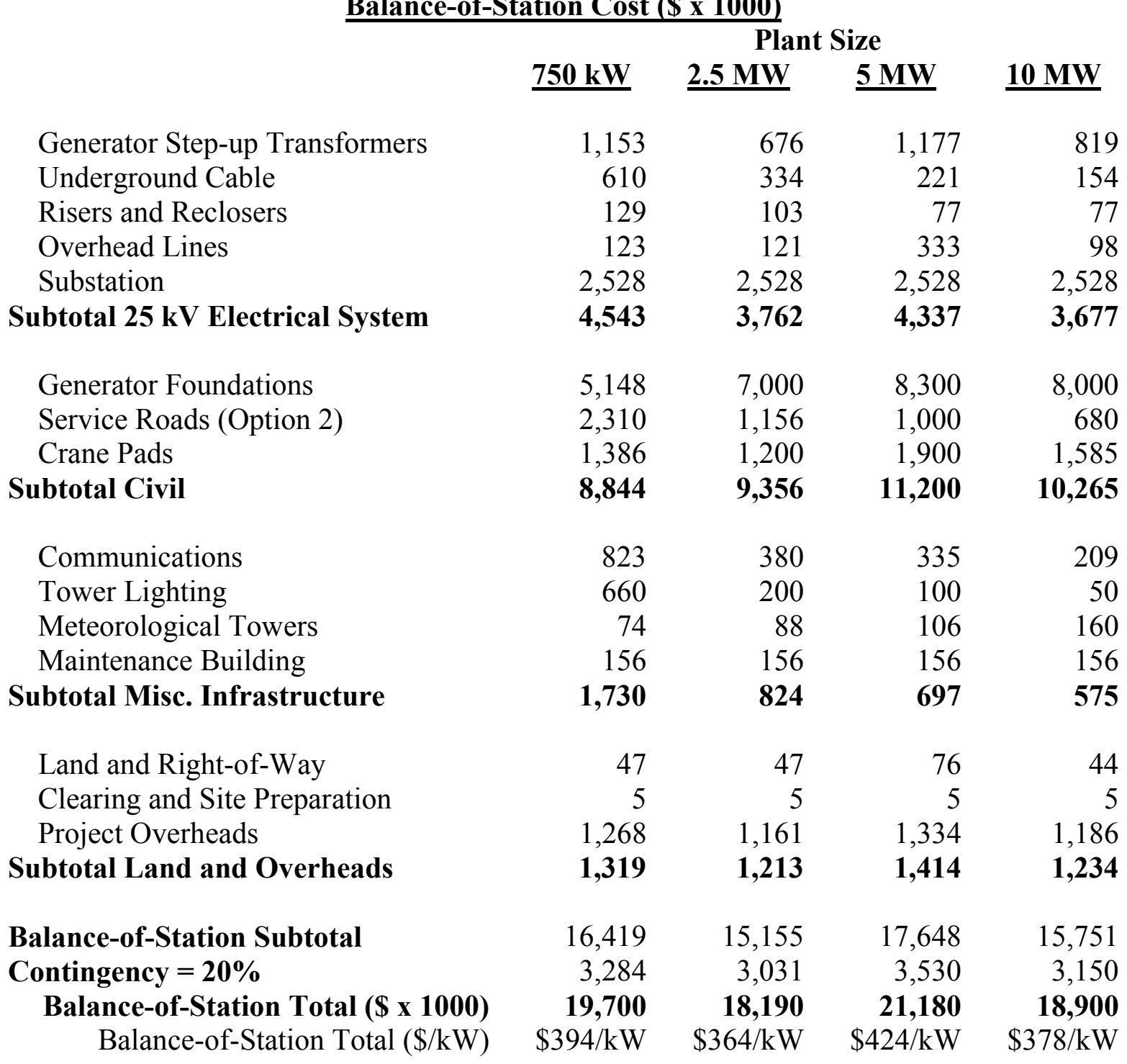

\section{Electrical-System Conclusions}

1. The electrical-power-collector system represents $23 \%$ to $27 \%$ of the balance-of-station cost. More than half of the electrical-collector system cost is the substation, which connects the wind farm to the existing power grid. The substation is designed for 50 MW, and its design and cost are independent of the wind turbine size.

2. The electrical-collector system comprises conventional utility equipment and facilities. All proposed sizes of wind turbine generators considered in this study are well within the current designs and capability of this equipment. Thus, there are no new technology or 
scaling issues with regard to the power system that would impede future application of larger-size wind turbines.

3. There is some economy of scale demonstrated in the electrical-system cost, especially in the 750 - to $2500-\mathrm{kW}$ size.

Chapter 3 provides additional details and conclusions on the electrical power system.

\section{Civil Conclusions}

1. The civil infrastructure cost comprises wind turbine foundations, access roads, and crane pads. These three items total $54 \%$ to $65 \%$ of the total balance-of-station cost, the majority of which comes from the wind turbine foundation.

2. The foundations and crane pads show an increasing unit cost for the larger turbine sizes, which identifies them as an area for additional study.

3. The size of access road and crane pad depends upon the size of crane needed for construction and maintenance. At the November 2 and 3, 2000, workshop, there was discussion on the use of self-erecting towers for the taller towers. This type of construction would help to minimize roadway width and crane pad sizes. Future research on this topic should be pursued.

4. More research needs to be done on foundation design and cost. We recommend that the foundation and tower be evaluated together. Modifications to the tower (greater diameter by flaring, or use of a four-legged tubular base) may result in much-reduced foundation costs.

Chapters 4 and 5 provide additional details and conclusions regarding foundations, roads, and crane pads.

\section{Miscellaneous Infrastructure Conclusions}

1. Miscellaneous infrastructure includes communications, tower lighting, meteorological towers, and the maintenance building, representing $10 \%$ of the total balance-of-station costs for the $66 \times 750-\mathrm{kW}$ wind farm, and a decrease to $4 \%$ for the $5 \times 10-\mathrm{MW}$ wind farm. The larger components of these costs for the $750-\mathrm{kW}$ wind farm are communications and tower lighting, which are a function of the number of towers. Because of the need for fewer towers, considerable cost savings can be achieved with the larger turbines.

2. We originally intended to study how maintenance building requirements and costs might vary with the larger turbine sizes. We never achieved this objective, however, because we were never able to quantify how the maintenance on a $5 \times 10-\mathrm{MW}$ wind farm might differ from that of the $66 \times 750-\mathrm{kW}$ wind farm. We believe the most practical means for maintaining the electrical power system and the communication system would be to 
contract with the local utility or a private contractor who does this type of work. For stocking of spare parts and maintenance on the wind turbines, there may be some economy by having one or more regionally located maintenance centers, which would have equipment and trained staff to call upon when needed rather than maintaining parts, equipment, and staff at each wind farm location. Our building cost is reflected in the minimal maintenance effort required at the wind farm.

Chapter 6 provides additional details and conclusions regarding miscellaneous infrastructure.

\section{Land, Overhead, and Contingency Cost Conclusions}

1. The land cost does not include land for the wind farm, but rather any land or land rights that would be required in addition to the wind farm. The land costs include land for the substation and the administrative building, as well as right-of-way for the power line between the substation and the wind farm. The clearing costs are minimal because the project is located in South Dakota.

2. The overhead costs include engineering, surveying, licensing, land acquisition, construction inspection, and owners' project management.

3. We included land, overhead, and contingency costs to provide an overall project total cost. 


\section{CHAPTER 3 ELECTRICAL SYSTEM}

\section{COST SUMMARY AND CONCLUSIONS}

The cost of the electrical system is divided into components, starting at the wind turbine and following the electrical system back to the interconnection with the utility transmission grid. The components are: generator step-up transformers (GSUs), underground cable, risers and reclosers, overhead line, and substation.

We studied two collector-system voltage levels for each of the four wind turbine sizes. For the $750-\mathrm{kW}$ size, 15 and $25 \mathrm{kV}$ were studied. For the other sizes, we studied 25- and 35-kV systems.

The costs for equipment and materials and construction labor for installation of the equipment and material for the electrical-collector system for the 50-MW wind farm are summarized in the following table. The costs, which are in today's dollars (\$ x 1000), do not include land; clearing; overhead costs such as engineering, surveying, and material procurement; or project management.

\begin{tabular}{|c|c|c|c|c|c|c|c|c|}
\hline \multirow{2}{*}{$\begin{array}{l}\text { System } \\
\text { Component }\end{array}$} & \multicolumn{2}{|c|}{$\begin{array}{c}\text { 750-kW } \\
\text { Plan }\end{array}$} & \multicolumn{2}{|c|}{$\begin{array}{l}\text { 2,500-kW } \\
\text { Plan }\end{array}$} & \multicolumn{2}{|c|}{$\begin{array}{l}\text { 5,000-kW } \\
\text { Plan }\end{array}$} & \multicolumn{2}{|c|}{$\begin{array}{c}\text { 10,000-kW } \\
\text { Plan }\end{array}$} \\
\hline & $15 \mathrm{kV}$ & $25 \mathrm{kV}$ & $25 \mathrm{kV}$ & $35 \mathrm{kV}$ & $25 \mathrm{kV}$ & $35 \mathrm{kV}$ & $25 \mathrm{kV}$ & $35 \mathrm{kV}$ \\
\hline GSU & 1,147 & 1,153 & 676 & 710 & 1,177 & 1,196 & 819 & 870 \\
\hline Cable & 643 & 610 & 334 & 356 & 221 & 237 & 154 & 174 \\
\hline Risers/Reclosers & 126 & 129 & 103 & 83 & 77 & 83 & 77 & 83 \\
\hline Overhead Line & 188 & 123 & 121 & 121 & 333 & 301 & 98 & 98 \\
\hline Substation & 2,612 & 2,528 & 2,528 & 2,729 & 2,528 & 2,729 & 2,528 & 2,729 \\
\hline Subtotal & 4,716 & 4,543 & 3,762 & 3,999 & 4,337 & 4,545 & 3,677 & 3,954 \\
\hline
\end{tabular}

\section{Conclusions: Electrical-Power-Collector System Costs}

1. The $25-\mathrm{kV}$ collector system was the least-cost plan for each wind turbine size studied, though there was no significant cost increase for the alternative voltage level. It might be beneficial to have the local utility maintain the electrical power system, in which case the best voltage for the collector system would be one that matches the local utility voltage.

2. The $750-\mathrm{kW}$ and $2,500-\mathrm{kW}$ plans show decreasing costs for the generator step-up transformers. These transformers are commonly used sizes and show a decreasing cost per unit. The generator step-up transformer for the 5,000-kW plan is at the upper limit of commonly used sizes, and the cost estimate reflects a higher cost per unit than the more common, smaller sizes. Similarly, for the 10,000-kW plan, a substation-type transformer was required. The total cost was lower than the $5,000-\mathrm{kW}$ plan because fewer units were required. 
3. The underground cable shows a decreasing cost for the larger turbine sizes because of shorter cable lengths and fewer terminations.

4. The reclosers show a declining cost for the larger turbine sizes because fewer reclosers were required.

5. The overhead line costs also show a general decline for the larger turbine sizes because of the shorter distance required. The 5,000-kW plan shows an anomaly because the layout required splitting the wind turbines to two locations, which necessitated doubling the length of overhead line over what would have been needed if the turbines were all located at one location.

6. The substation is the dominant cost component of the electrical-collector system. This cost is independent of wind turbine size.

7. The study confirmed that conventional utility equipment and design practices could be used for the electrical-power-collector system for all sizes of wind turbines.

8. There is some economy of scale with regard to larger wind turbine sizes. The cost for the electrical-collector system for a $50-\mathrm{MW}$ wind farm for the $750-\mathrm{kW}$ size is approximately $\$ 91 / \mathrm{kW}$. This reduced to $\$ 75 / \mathrm{kW}$ and $\$ 74 / \mathrm{kW}$ for the $2.5 \mathrm{MW}$ and $10 \mathrm{MW}$ turbines, respectively. These do not include costs for land, clearing, and overhead.

\section{DESIGN GUIDELINES AND ASSUMPTIONS}

The following design guidelines were established as the basis of the study of the electricalcollector systems.

1. Conventional utility-grade equipment will be used to interconnect the wind turbines. Typical medium-voltage equipment used by utilities is rated 15,25 , or $35 \mathrm{kV}$. We evaluated two voltage levels for each size wind turbine. We studied $25 \mathrm{kV}$ for all plans. Alternatives using $15-\mathrm{kV}$-class equipment for the $750-\mathrm{kW}$ plan and $35 \mathrm{kV}$ for the $2,500-$ $\mathrm{kW}, 5,000-\mathrm{kW}$ and $10,000-\mathrm{kW}$ plans were considered to determine if there might be a significant cost difference for the alternative voltage.

2. Under the umbrella of $15-\mathrm{kV}$-class equipment, different utilities have standardized on different operating voltages; for example, 12.47, 13.2, and $13.8 \mathrm{kV}$. For this study, we chose $13.8 \mathrm{kV}$ as our operating voltage for the $15-\mathrm{kV}$ plan. Similarly, for the $25-$ and $35-$ $\mathrm{kV}$-class equipment, we chose 24.9 and $34.5 \mathrm{kV}$ as the operating voltages.

3. The generator step-up transformer will be designed to match the generator voltage on the low-voltage side and to match the medium-voltage system on the other side. One consideration is the impact of the generator voltage on the collector-system costs. The transformer manufacturers have considerable control and latitude with regard to the design of the low-voltage winding of the transformer. There should not be a significant cost difference in the generator step-up transformers as long as the voltages are within 
typical ranges. We arbitrarily chose typical voltages of $480 \mathrm{~V}$ for the $750-\mathrm{kW}$ wind turbines, $690 \mathrm{~V}$ for $2.5 \mathrm{MW}, 2,400 \mathrm{~V}$ for $5 \mathrm{MW}$, and 4,160 V for the 10-MW size. Optimization of the generator voltage should be based on optimization of the generator and associated power electronics design; therefore, we do not consider it a design issue for the collector system.

4. The generator step-up transformers will be padmount transformers with all cables entering the transformer underground. Standard padmount transformers will be used as follows:
a. $\quad 750 \mathrm{~kW}-1,000-\mathrm{kVA}$ transformer
b. $\quad 2,500 \mathrm{~kW}-3,000-\mathrm{kVA}$ transformer
c. $\quad 5,000 \mathrm{~kW}-7,500-\mathrm{kVA}$ transformer

Because 7,500 kVA is the largest standard padmount transformer, the $10,000-\mathrm{kW}$ wind turbines will be provided with a 10/12.5-MVA substation transformer with separate padmounted switchgear installed on the high side of the transformer.

5. The medium-voltage cables, which comprise the power system between and around the wind turbines, will be underground to maintain visual aesthetics. Standard utility-grade underground cable will be used. Utilities use three types of construction for underground systems: direct-bury cable, direct-bury cable in conduit, and cable installed in concreteencased duct bank. Concrete-encased duct bank is the most expensive and is used in urban areas where there is high probability of someone digging into the cable and where expensive streets and sidewalks exist over the duct bank. The duct bank protects the cable and allows a failed cable to be removed and a new cable installed without disturbing the activities or facilities above. Cable in a buried conduit is the next most expensive option, because a conduit is required in addition to the cable. The conduit provides some protection to the cable, and allows a cable to be replaced without digging it up. The least expensive option with regard to first-installation cost is to directly bury the cable. The cable manufacturer warrants the direct-buried cable the same as if the cable is installed in conduit. The disadvantage of direct-buried cable is that it is more likely to be damaged if someone digs into it. Also, if a direct-buried cable fails, it is necessary to dig up the cable at the location of the failure to repair it. For the purpose of this study, we are assuming direct-buried cable.

6. The underground cable would connect from one windmill to the next until a convenient roadway location is encountered, at which point the underground cable would be connected to a main collector line. For the main collector line, we assumed that a conventional three-phase overhead distribution line would be built, and that the line would be built along a roadway, as is the practice of the local utility. Thus, the overhead line would aesthetically blend into the landscape as "just another distribution line."

7. At the interface between the underground and the overhead collector lines, we have assumed a fault-interrupting device called a "recloser." This is a conventional utility "circuit breaker" that is used to isolate a section of the line automatically should there be 
an electrical fault. Each string of windmills would connect to the overhead main by a recloser. Thus, should there be an underground cable or transformer failure, only those windmills connected together on the string would be disconnected. All other windmills would continue to operate and remain connected to the main line.

8. We assumed that the existing utility substation serving the area cannot be used to connect the collector system to the $115-\mathrm{kV}$ line. Therefore, cost estimates are provided for a new 30/40/50-MVA, 115-kV distribution substation to make this connection.

\section{ELECTRICAL-SYSTEMS ANALYSIS}

We analyzed power flow to verify the feasibility of the proposed $15-\mathrm{kV}$ plan to interconnect $66 \mathrm{x}$ $750-\mathrm{kW}$ generators as described above. Likewise, power flow analyses were performed to verify the feasibility for the $24.9-\mathrm{kV}$ and $34.5-\mathrm{kV}$ plans for the four sizes of wind turbines. The power flow analysis calculates the line flows, $\mathrm{kW}$ and $\mathrm{kVAR}$ line losses, line voltage drops, and VAR requirements at each generator. The assumptions and data for this analysis are provided in Appendices A and B. The power flow results are provided in Appendix C.

We conducted a short-circuit analysis for the proposed plans. The short-circuit analysis determines the expected fault levels, and is used to assure that the equipment will operate as intended. Because the results were similar for all plans, only the results for the $750-\mathrm{kW}$ turbines at $15 \mathrm{kV}$ are shown in Appendix D. 


\section{CHAPTER 4 FOUNDATIONS}

To calculate foundation costs, the wind turbine tower was assumed to be a single shaft of the heights and diameters shown in the table below. The wind turbine mass, overturning moment, and base diameter are factors used to determine the costs of the foundation. The rotor diameter, hub height, and base diameter increase about three-and-one-half times for the range of plant sizes from 750 to $10,000 \mathrm{~kW}$. However, the total mass and overturning moment increase by 30 to 40 times.

\begin{tabular}{|c|c|c|c|c|c|}
\hline $\begin{array}{c}\text { Plant Size } \\
\mathbf{k W}\end{array}$ & $\begin{array}{c}\text { Rotor } \\
\text { Diameter }\end{array}$ & Hub Height & Base Diameter & $\begin{array}{c}\text { Total } \\
\text { Mass* } \\
\text { kg }\end{array}$ & $\begin{array}{c}\text { Overturning } \\
\text { Moment } \\
\text { kN-m }\end{array}$ \\
\hline 750 & $50 \mathrm{~m}(164 \mathrm{ft})$ & $65 \mathrm{~m}(213 \mathrm{ft})$ & $3.7 \mathrm{~m}(12.1 \mathrm{ft})$ & 104,927 & 38,718 \\
\hline 2,500 & $85 \mathrm{~m}(279 \mathrm{ft})$ & $111 \mathrm{~m}(364 \mathrm{ft})$ & $6.4 \mathrm{~m}(21 \mathrm{ft})$ & 466,061 & 190,185 \\
\hline 5,000 & $120 \mathrm{~m}(394 \mathrm{ft})$ & $156 \mathrm{~m}(512 \mathrm{ft})$ & $9.0 \mathrm{~m}(29.5 \mathrm{ft})$ & $1,237,715$ & 535,070 \\
\hline 10,000 & $170 \mathrm{~m}(558 \mathrm{ft})$ & $221 \mathrm{~m}(725 \mathrm{ft})$ & $12.7 \mathrm{~m}(41.7 \mathrm{ft})$ & $3,338,895$ & $1,521,106$ \\
\hline
\end{tabular}

The foundation costs can vary significantly depending on soil conditions, rock, and proximity of concrete supplies. To compare foundation designs and costs, a square pad-and-pier foundation geometry was assumed (Figure 11). As shown in the table below, the foundation width increases from 42 to 130 feet and the volume of concrete increases from 340 cubic yards for the $750-\mathrm{kW}$ size to 7,250 cubic yards for the 10-MW size. The cost for each foundation increases from $\$ 78,000$ to $\$ 1,600,000$. These estimates can be $40 \%$ to $50 \%$ conservative.

$\begin{array}{cccccc}\begin{array}{c}\text { Plant Size } \\ \mathbf{k W}\end{array} & \text { Width } & \begin{array}{c}\text { Concrete } \\ \mathbf{m}^{3}(\mathbf{C u} . \mathbf{y d} .)\end{array} & \begin{array}{c}\text { Cost Each } \\ \mathbf{\$ x 1 , 0 0 0}\end{array} & \begin{array}{c}\text { Number of } \\ \text { Foundations }\end{array} & \begin{array}{c}\text { Total Cost } \\ \mathbf{\$ x 1 , 0 0 0}\end{array} \\ 750 & 13 \mathrm{~m}(42 \mathrm{ft}) & 260(340) & 78 & 66 & 5,148 \\ 2,500 & 21 \mathrm{~m}(70 \mathrm{ft}) & 1,185(1,550) & 350 & 20 & 7,000 \\ 5,000 & 30 \mathrm{~m}(100 \mathrm{ft}) & 2,944(3,850) & 830 & 10 & 8,300 \\ 10,000 & 40 \mathrm{~m}(130 \mathrm{ft}) & 5,543(7,250) & 1,600 & 5 & 8,000\end{array}$

We also considered a rock-anchor foundation (Figure 14), which is required only when bedrock is close to the surface. The estimated cost for the rock-anchor design is slightly higher than that for the pad-and-pier design; again, however, the estimate is conservative.

Alternative foundation concepts would include cylindrical designs. These designs have implications with regard to excavating and forming, which makes it more difficult to provide cost estimates without knowing soil conditions.

There was discussion at the November 2 and 3 workshop that these foundation costs (for the $750-\mathrm{kW}$ size) may be approximately twice those of existing installations. As stated previously, the costs given are conservative, but they are relative. We believe the location of the site with 
regard to soil conditions and proximity to concrete supplies may result in a large variation in costs for foundations.

\section{Conclusions on Foundation Costs}

1. The foundation cost, based on extrapolating the existing design of a single-shaft tower, results in an increasing unit cost for the larger sizes.

2. It is difficult to optimize the foundation design independent of the tower design. It would be better to consider the tower and foundation together in future studies. 


\section{CHAPTER 5 OTHER CIVIL INFRASTRUCTURE}

\section{ROADS}

We assumed that maintenance and construction roadways would be located along the line of turbines parallel to the cable routes shown on the layout drawings. The cost includes labor and material to build the roadways, including culverts. The cost does not include overhead costs, such as engineering, surveying, and management. In addition, we assumed that county or township roads would exist in the vicinity of the wind farm, and that the only wind farm roadways would be those between the turbines, as shown on the layout drawings. No costs have been included for paving or upgrading the county or township roads.

We considered three alternative road concepts. Option 1 would be a minimal, 20-foot-wide gravel road. This assumes that construction and maintenance access is not needed for heavy and large equipment. In other words, the towers and heavy equipment would be shipped in small pieces and assembled and erected at each site. Any equipment too big or heavy to be moved on a minimal road would have to be moved by helicopter or by building temporary roads.

Option 2 would be a paved road wide enough for moving cranes and maintenance equipment but not sufficient for equipment (cranes) that may be needed for the initial construction of the towers and rotors. If a very large crane is needed for initial construction, it might need to be disassembled to move it from one tower site to the next.

Option 3 would be a paved road wide enough for moving the largest crane from site to site for the initial erection of towers and rotors without disassembly of the crane.

\begin{tabular}{|c|c|c|c|c|c|c|c|}
\hline \multirow{2}{*}{$\begin{array}{l}\text { Plant } \\
\text { Size } \\
\text { kW }\end{array}$} & \multirow{2}{*}{$\begin{array}{c}\text { Total } \\
\text { Length } \\
\text { mx1,000 } \\
(\mathbf{f t x} 1,000)\end{array}$} & \multicolumn{2}{|c|}{$\begin{array}{c}\text { Option 1 } \\
\text { Gravel Road } \\
\end{array}$} & \multicolumn{2}{|c|}{$\begin{array}{c}\text { Option } 2 \\
\text { Paved Road } \\
\end{array}$} & \multicolumn{2}{|c|}{$\begin{array}{c}\text { Option } 3 \\
\text { Paved Road } \\
\end{array}$} \\
\hline & & $\begin{array}{l}\text { Width } \\
\text { m (ft) }\end{array}$ & $\begin{array}{c}\text { Cost } \\
\$ \times 1,000\end{array}$ & $\begin{array}{l}\text { Width } \\
\text { m (ft) }\end{array}$ & $\begin{array}{c}\text { Cost } \\
\$ \times 1,000\end{array}$ & $\begin{array}{l}\text { Width } \\
\text { m (ft) }\end{array}$ & $\begin{array}{c}\text { Cost } \\
\$ \times 1,000\end{array}$ \\
\hline 750 & $7.8(25.6)$ & $6(20)$ & 1,290 & $8(26)$ & 2,310 & $9(30)$ & 2,830 \\
\hline 2,500 & $3.9(12.8)$ & $6(20)$ & 643 & $8(26)$ & 1,156 & $9(30)$ & 1,413 \\
\hline 5,000 & $2.8(9.1)$ & $6(20)$ & 460 & $10(32)$ & 1,000 & $12(40)$ & 1,270 \\
\hline 10,000 & $1.9(6.1)$ & $6(20)$ & 310 & $10(32)$ & 680 & $12(40)$ & 870 \\
\hline
\end{tabular}

\section{Conclusions on Roads}

1. The maintenance and access road costs show a decreasing cost for the larger turbine sizes because considerably less length is required for these sizes. As mentioned above, this cost estimate does not include any work that may be needed on the county or township roads to accommodate the larger turbines. This issue is addressed in the Technical Area 2 study. 


\section{CRANE PADS}

Crane pads are another related cost. If a large crane is needed to erect the tower and rotor, then a crane pad may be needed for safe operation of the crane. The estimated size and cost of the crane pads are summarized below.

\begin{tabular}{|c|c|c|c|c|c|}
\hline $\begin{array}{c}\text { Plant Size } \\
\mathbf{k W}\end{array}$ & $\begin{array}{c}\text { Area } \\
\mathbf{m}^{2}\left(\mathrm{ft}^{2}\right)\end{array}$ & $\begin{array}{c}\text { Concrete } \\
\text { m }^{3} \text { (cu.yd.) }\end{array}$ & $\begin{array}{c}\text { Cost Each } \\
\$ \times 1,000\end{array}$ & No. Pads & $\begin{array}{c}\text { Total Cost } \\
\$ \times 1,000\end{array}$ \\
\hline 750 & $243(2,616)$ & $81(106)$ & 21 & 66 & 1,386 \\
\hline 2,500 & $1,000(10,764)$ & 175 (299) & 60 & 20 & 1,200 \\
\hline 5,000 & $2,400(25,834)$ & 732 (957) & 190 & 10 & 1,900 \\
\hline 10,000 & $4,000(43,056)$ & $1,220(1,595)$ & 317 & 5 & 1,585 \\
\hline
\end{tabular}

\section{Conclusions on Crane Pads}

1. If crane pads are required to erect the very large towers, not only is the cost significant, but it also increases for the larger plants. 


\section{CHAPTER 6 MISCELLANEOUS INFRASTRUCTURE}

\section{COST SUMMARY AND CONCLUSIONS}

Miscellaneous infrastructure includes communications, tower lighting, meteorological towers, and the administrative building. For data acquisition and control, the communications fiber-optic system is routed between the administrative building and each wind turbine, the electrical reclosers, and the meteorological towers. The tower lighting consists of aircraft warning lighting located on top of each wind turbine tower. The meteorological towers provide wind and other weather data. The administrative building provides housing for the wind farm administrative and maintenance functions on-site.

\section{System Component}

Communications

Tower Lighting

Meteorological Towers

Maintenance Building

\section{$750 \mathrm{~kW}$}

823

660

74

156

$\$ 1,713$

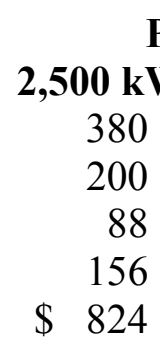

Plant Size
$5,000 \mathrm{~kW}$

335

100

106

156

\$ 697
$10,000 \mathrm{~kW}$

209

50

160

156

$\$ 575$

\section{Conclusions on Miscellaneous Infrastructure:}

1. The communications costs are a function of total linear distance from the administrative building to each wind turbine, as well as the number of turbines. As shown, there is a significant cost saving in the larger turbine plan because there are fewer turbines and shorter distances involved.

2. Tower lighting is a function of number of turbines and favors the larger wind turbines because fewer towers are needed.

3. Only two meteorological towers are required for the wind farm site regardless of the size and number of turbines for the 50-MW wind farm studied. The larger wind turbines require higher meteorological towers, resulting in increasing cost for the meteorological towers as wind turbine size increases.

4. We estimate that the cost of the administrative building is independent of wind turbine size.

5. Considering the sum of the miscellaneous items, the larger turbine sizes demonstrate a decreasing cost for a 50-MW wind farm. 


\section{COMMUNICATIONS SYSTEM}

Figure 10 shows a one-line diagram of a communications system that would apply to plans for any of the proposed generator capacities. The proposed communications system consists of a master station located in the administrative building connected to remote terminal units at each of the generators, reclosers, and meteorological towers by way of multiple overhead and underground fiber-optic circuits. Costs for the substation include providing the local utility with output data from the wind farm's communications system by way of a fiber-optic circuit.

\begin{tabular}{|c|c|c|c|c|c|c|}
\hline \multicolumn{7}{|c|}{ Length } \\
\hline $\begin{array}{c}\text { Plant Size } \\
\mathbf{k W}\end{array}$ & $\begin{array}{c}\text { Number } \\
\text { RTU* }\end{array}$ & $\begin{array}{c}\text { RTU Cost } \\
\$ \times 1,000\end{array}$ & $\begin{array}{c}\text { Fiber } \\
\mathbf{m x 1 , 0 0 0} \\
(\mathbf{f t x} 1,000)\end{array}$ & $\begin{array}{c}\text { Fiber Cost } \\
\$ \times 1,000\end{array}$ & $\begin{array}{l}\text { Master } \\
\text { Station } \\
\text { \$x1,000 }\end{array}$ & $\begin{array}{c}\text { Total Cost } \\
\$ \times 1,000\end{array}$ \\
\hline 750 & $66+5+2$ & 588 & $10.3(33.9)$ & 168 & 67 & 823 \\
\hline 2,500 & $20+3+2$ & 201 & $6(19.6)$ & 104 & 67 & 372 \\
\hline 5,000 & $10+3+2$ & 121 & $7.4(24.4)$ & 148 & 67 & 335 \\
\hline 10,000 & $5+3+2$ & 81 & $3.4(11.1)$ & 62 & 67 & 209 \\
\hline
\end{tabular}

* Based on number of turbines + number of reclosers + number of meteorological towers.

\section{TOWER LIGHTING AND METEOROLOGICAL TOWERS}

Tower lighting is provided on each wind turbine tower as required by Federal Communications Commission (FCC) regulations for aircraft warning.

Two meteorological towers are required for the 50-MW wind farm site regardless of the number of turbines. The tower height for the meteorological tower is based on approximately $83 \%$ of the hub height.

$\begin{array}{ccc}\text { Plant Size } & & \text { Cost } \\ \mathbf{k W} & \text { No. } & \mathbf{\$ x 1 , 0 0 0} \\ 750 & 66 & 660 \\ 2,500 & 20 & 200 \\ 5,000 & 10 & 100 \\ 10,000 & 5 & 50\end{array}$

\begin{tabular}{ccc}
\multicolumn{3}{c}{$\begin{array}{c}\text { Meteorological Towers } \\
\text { Height }\end{array}$} \\
No. & m & $\begin{array}{c}\text { Cost } \\
\mathbf{\$ x 1 , 0 0 0}\end{array}$ \\
2 & 54 & 74 \\
2 & 92 & 88 \\
2 & 130 & 106 \\
2 & 180 & 160
\end{tabular}




\section{MAINTENANCE BUILDING}

\section{Description}

2,400-Square-Foot Service Building

Water

Septic

Driveway/Parking

Security Fence

Gates

\section{Total}

100,200

5,000

10,000

5,400

32,400

2,900

Subtotal

155,900

\$ 156,000

15,000

0

2,400

Subtotal (Rounded)

Site Preparation

Land (Acres)

Site Clearing

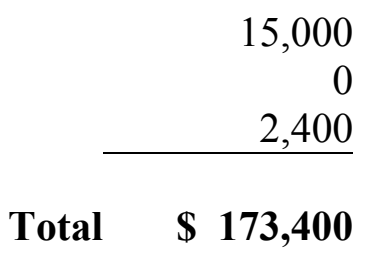

Total $\$ \mathbf{1 7 3 , 4 0 0}$

We have not determined how the maintenance building requirements might vary as a function of turbine size. The building costs we have provided are for a minimal building. We used the same building cost for all four plans. This is one item that would benefit from additional effort, though we do not expect that there are any new technology or research items associated with this effort. Furthermore, we do not expect this to be a major cost item. However, it does need to be included in the overall balance-of-station cost for computing the cost of wind energy.

For a utility, a 50-MW plant is considered small. Economic considerations dictate keeping the operating and maintenance costs for a $50-\mathrm{MW}$ plant as low as possible. An area of future research might be to consider building a regional operating and maintenance facility that would be responsible for many 50-MW wind farms. This might eliminate the need for an administrative and maintenance building (and the need for staff and equipment) at each wind farm site. If space for on-site maintenance or storage were needed, one possibility would be to use one or more specially designed semi-trailers. These could be constructed in a factory and provided to as many wind farm sites as needed. 


\section{CHAPTER 7 QUESTIONS AND ANSWERS}

Commonwealth Associates, Inc. (CAI) prepared a draft report in June 2000. Questions with regard to that report and our responses are provided in this chapter.

1. The turbine layout seems somewhat simplistic with regard to terrain. Wouldn't the layout follow more of the contours of the land, particularly in the layout for the $750-\mathrm{kW}$ turbine, where the second (downwind) row is below the ridgeline? A layout that conforms more to the land may result in changes in inter-turbine electric-line lengths. How was the spacing of 2.3 rotor diameters between turbines, and 12 rotor diameters between rows, determined?

The layouts have been constructed based on wind roses from Pierre, South Dakota, and Valentine, Nebraska, applied to the research of Germain and Bain ("Economics of Wind Farm Layout," Proceedings of WindPower '97). The wind roses show a strong predominance of energy from the northwest and northnorthwest. The turbines have been oriented perpendicular to an assumed prevailing wind direction of $343^{\circ}$ to best utilize the natural landforms in the study area north of Mission, South Dakota. The wind roses from sites in this area of the country will share very similar characteristics. Sites from Kansas southward may have strong southern prevailing winds, and sites in Iowa may have bi-modal south-northwest flow, but sites in the Dakotas and Nebraska should have very similar characteristics to the Pierre and Valentine data referenced here.

Taking the distribution of wind energy by sector and interpolating between the Isotropic and Preferred Direction results of Tables 4 and 5 from Germain and Bain, the spacing for lowest cost of energy was determined to be 2.3 rotor diameters between turbines and 12 rotor diameters between rows.

A rectilinear-array approach has been taken in this case for three reasons. First, the gentleness of the terrain allows for array layouts to be constructed without regard for topography. Second, the general scaling nature of the WindPACT study makes rectilinear layouts desirable to facilitate linear interpolation of results to intermediate turbine sizes. Third, site-specific layout optimization is beyond the scope of this project. However, some site-specific tailoring of the rectilinear layouts has been done to make them more realistic.

Actual project implementation would typically not include rectilinear turbine layout, but would instead utilize numerical simulation programs such as WindFarmer or WindPro. These programs use complete descriptions of the wind resource by speed and direction, digital terrain data, property boundary information, and setback distances as inputs into a numerical optimization routine that determines the layout that maximizes the energy output. These layouts group turbines in the highest-energy-production regions, which are 
typically the highest-elevation sites. Because the terrain in the study area does not present a large enough feature perpendicular to the prevailing flow to contain the required number of turbines, the model would probably utilize a larger spacing between turbines. The space between rows would not be a relevant parameter because the model would not assume the turbines are arranged in rows.

2. Would changing the inter-turbine spacing to more than the 2.3 diameters affect the conclusions? It seems a bit tight, though some commercial installations in Texas seem to be using this spacing, whereas wind farms in Minnesota and Iowa are not.

Changing the spacing between turbines would change the cost of cable, the cost of roads, and the cost of communication fiber optics. Also, changing distance between rows could increase the length of the overhead line. Approximate unit costs for these items are provided in Tables E2.1 through E2.4. We would expect increased spacing would impact the $66 \times 750-\mathrm{kW}$ plan more than wind farms with the larger turbines. We concluded that these items demonstrate decreasing unit costs for the larger turbine wind farms. Thus, our conclusion would not change if we had spaced the turbines farther apart.

3. Why are three of the 5-MW turbines located so far from the substation compared to the $750-\mathrm{kW}$ plan? Were closer alternatives considered?

Three of the 5-MW turbines were located at a distance from the others due to the spacing assumptions explained in question \#1 above. Closer alternatives were not examined.

4. Can you relate the costs to installed $\mathrm{kW}$ and swept area to allow observation of implications to cost of energy?

The cost tables compare and summarize the balance-of-station costs (in today's dollars) for the four sizes of wind turbines. All four plans provide a wind farm with a total of $50 \mathrm{MW}$ and presumably the same annual energy production. Thus, relative ranking of the plans is the same whether you compare the plans on total dollars, dollars per $\mathrm{kW}$, or dollars per $\mathrm{kWh}$. We have added a dollars per $\mathrm{kW}$ number on the bottom of the summary table in Chapter 2.

5. Why do the $25-\mathrm{kV}$ electrical plans turn out cheaper for each of the turbine sizes? Some explanation would be helpful.

The industry uses three standard voltages for power distribution equipment and cable that are applicable for building the wind farm collector system: 15, 25, and $35 \mathrm{kV}$. Also, the industry standard ratings for underground cable terminations are 200 and 600 amps. Based on unit costs for these standard components, the distances involved, and the power to be transported, the 25-kV plan has a slight cost advantage, as our calculations show. Under different assumptions with regard to power and distance, another voltage may be better. 
6. The $\mathrm{X} / \mathrm{R}$ ratio for substation transformers appears too large. Please review and comment.

Table A2 lists our X/R assumption of nine for the generator step-up transformers. In Appendix $B$ we used 19 for the 30-MVA substation transformer. The X/R ratio is used to calculate the resistance of the transformer for power flow and shortcircuit modeling. Institute of Electrical and Electronics Engineers (IEEE) C37010-1979 and IEEE 141-1993 provide the following typical X/R ratios for transformers:

$$
\begin{aligned}
& \text { 1,000 kVA }-7, \text { range not available } \\
& 5,000 \mathrm{kVA}-12,5-18 \\
& 10,000 \mathrm{kVA}-15,6-20 \\
& 30,000 \mathrm{kVA}-23,11-30
\end{aligned}
$$

Our assumed values are within the range of transformer $X / R$ ratios and, perhaps, too low with regard to typical values. The transformer resistance is a design function. A higher $X / R$ ratio implies a lower resistance and, hence, a more efficient transformer. Thus, our calculations of losses may be a pinch high. The calculation of load losses is provided as part of the power flow simulation. We tabulated these numbers and reviewed them for reasonableness; however, they do not directly impact the study conclusions. Because we believe the resistance values we assumed do not affect our study, we recommend no changes in our assumptions at this time.

We would like to offer one additional comment with regard to transformer design and efficiency. We did not model or tabulate transformer no-load losses. This is also a design variable that the manufacturer can modify. The no-load loss is present any time that the transformer is energized; presumably, this would be $100 \%$ of the time (except when the transformer is out of service for maintenance). On the other hand, the load losses, which are a function of transformer resistance, will be less of a factor because of the low capacity factor of the wind generators. A candidate for a future study would be an economic evaluation of transformer costs versus no-load and load losses.

7. The wind farm is expected to be composed of variable-speed wind turbines. Thus, the wind turbine generator will be a current source generator (with power converter). This would reduce the need for capacitors and power factor correction, and reduce shortcircuits. In the event of a short-circuit, the current output of the generator will be automatically limited within a half cycle by the power electronics.

The power flow and short-circuit modeling was done to confirm that the proposed collector system would indeed work as we intended. With the power flow model, we wanted to determine the expected voltage drop across the system from no generation to full generation. Our criteria were to have the voltage within plus or minus $5 \%$ of the substation bus voltage, over the range of no generation to full 
generation. To model the generator, we assumed that the power electronics could be designed to provide any reasonable power factor at the machine. For this firstcut model, we set generators to hold $1.00 \mathrm{pu}$ voltage on their terminals within the limits of plus or minus 95\% power factor. We also considered the line-charging kVAR of the underground cable (though this proved to be negligible). The results of the power flow simulations confirmed that we did indeed have a viable highvoltage collector system, with a flat voltage profile from no generation to full generation. Also, we determined that some generators were operating at slightly leading and others at slightly lagging power factors, and that all were well within the plus or minus 95\% power factor limit. From this we conclude the following: that there should be no problem in designing the power electronics to provide sufficient kVAR at each machine as necessary to regulate the voltage, and that the machine terminal voltage can serve as the control variable of the kVAR at each machine (i.e., simple local control of each machine).

From the above calculations, we determined that we have an acceptable voltage profile across our collector system and, consequently, we will not need a voltage regulator or load-tap-changing (LTC) transformer at our substation. This is good news from two perspectives. First, we save the cost of this equipment, and second, LTC transformers are mechanical devices that can be a maintenance problem with frequent operation. (We do not have experience with any actual wind farms; however, our seat-of-the-pants expectation is that the output of the wind plant may change frequently and, thus, cause many LTC operations, leading to a maintenance concern.)

The power flow model provides a calculation of the $\mathrm{kW}$ and $\mathrm{kVAR}$ losses on the high-voltage collector system. We determined that our collector system would have approximately 10-MVAR losses at full generation. These losses are a function of the square of the current flow on the collector system. Hence, the MVAR losses will be expected to change even more rapidly than the generation. Depending upon the relative stiffness of the transmission system, a 10-MVAR fluctuating load on the utility system may very likely cause the utility transmission voltage in the vicinity to fluctuate. For example, the voltage at the Mission Distribution Substation may be affected. If so, the voltage regulator or LTC at Mission would continually be operating in an attempt to hold voltage, thus leading to more frequent maintenance. Because of these types of problems, we would expect the utility to insist that the wind farm supply power to the system at unity power factor.

Although it is possible to design a control system that would provide these MVAR losses from the wind machines themselves, we believe a simpler and more direct approach is to provide the MVAR losses by capacitors at the substation. Because we expect the capacitors will need to be switched frequently, we would propose to use power electronic switching rather than mechanical switching. The control of the switching would be the power factor at the substation. A commercial product that provides this type of capacitor switching and control is on the market. We 
believe this would be the most cost-effective approach to providing unity power to the utility.

We included the cost of this device in our estimates of the substation. It is the same for all plans. We recommend that we keep this feature in our report, as we presently have it.

The other comment above deals with the short-circuit contribution of the generator power electronics. We prepared a short-circuit model of the power system to determine if we can apply standard distribution-type equipment, primarily the reclosers. We are using a recloser as the switching device between the overhead and underground cables. We are proposing to use this device because it is standard equipment familiar to utility maintenance people, it can be used as a fault-interrupting device, it can be used as a remotely controlled switching device, it is a three-phase switching device, it is lower in cost than a full-fledged circuit breaker, and it can provide data to the supervisory control and data acquisition (SCADA) system. The standard short-circuit rating of this device is $12 \mathrm{kA}$. We did not have a good short-circuit model for the power electronic wind machines, so we just modeled them as conventional generators to get an order-of-magnitude feel for the approximate fault levels we might expect. We determined that the greatest contribution to the fault current was not the generators, but rather the power system. We concluded that we could use standard reclosers for most applications except very close in to the substation. Thus, it was not necessary for us to have a more sophisticated short-circuit model of the generators. We believe the assumptions and calculations in our report are adequate for the purpose intended.

8. Did the design consider harmonics mitigation for the static VAR compensation?

One form of static VAR compensator uses power electronics to provide continuous control of current through a reactor, which is in parallel with a capacitor. The VAR output can be designed for a range of both lagging and leading VARs, depending upon the size of the reactor and capacitor. The power electronics can provide very fast response and virtually any output within the design limits. These types of devices are ideally suited for compensating rapidly fluctuating VAR loads, such as those encountered with arc furnaces and welding machines. The disadvantages of these devices are:

a) They generate harmonics (usually mitigated by design of the capacitor banks, which provide leading VARs and act as harmonic filters).

b) They are expensive.

The capacitors that we are proposing are not a static VAR compensator as defined above. Instead, they are standard distribution-type capacitors, which are switched by power electronics. One supplier of this type of equipment is Power Quality Systems, Inc. More information on their product is provided at www.pwrqualitysys.com. The power electronics cause switching on the zero axis 
crossing of the current to minimize harmonic generation. No harmonic mitigation would be required.

9. What would be the impact on total facility cost of radio communications to a central location and cellular communications to outside of the wind park?

Our preliminary estimate for radio communications is $\$ 3,000 /$ Remote Terminal Unit (RTU) (at each wind turbine) and \$20,000 for the Master Station. For $66 x$ 750-kW wind turbines, the cost for radio is slightly higher than our estimated cost for fiber-optic communication. However, for the other three larger turbines (fewer units), the preliminary cost for radio is significantly less (approximately $50 \%$ ) than the cost for fiber. These preliminary numbers indicate that additional work to evaluate both fiber-optic and radio communications may be warranted.

Cost for cellular is essentially the cost of a Remote Master communicating with the Master (estimated at \$25,000).

10. Why are crane pads required? Have not seen them used in many, if any, wind farms (at least up to $1.6 \mathrm{MW}$ ).

Crane pads are required if the site is not already level and does not provide sufficient soil-bearing capacity for the crane to be used. Cost estimation of crane pad construction will depend on the soil-bearing requirements of the particular crane, and the bearing strength of the native soil. The "Vestas Standard Civil Specifications" for the $V-47(660 \mathrm{~kW})$ turbine require that crane pads be constructed with an area of 139-260 $\mathrm{m}^{2}$. (We used $243 \mathrm{~m}^{2}$.) The crane pads are required to provide a bearing capacity of $3,000 \mathrm{lb} / \mathrm{ft}^{2}$ with a Standard Proctor (percentage compaction) of $95 \%$ and a maximum elevation variation of 2 " across the pad.

11. Overhead collector lines between turbines and substation would most likely be maintained by the project owner/operator, not by a local utility. In addition, considering the project's location, utilizing the overhead poles for distribution to customers appears unlikely.

It will probably be cheaper to contract with the local utility to maintain the overhead and underground high-voltage system than to attempt to maintain trained staff, maintenance equipment, and spare parts yourself. That is one of the reasons we were careful to use conventional utility-type equipment in the design of the collector system. We agree that, for the South Dakota site, there may be little opportunity for joint use of the overhead poles to service utility customers. However, as a general practice, offering access to electrical power where it previously was not available (except at the additional cost to build a power line) may be added economic incentive to the landowner with whom you are negotiating to obtain the easement for your power line. 
12. Did you consider lightning protection for the overhead line?

Our concept design uses the neutral placed above the phase conductors to serve the dual purpose of neutral and lightning shield wire on the overhead line. We have also shown lightning arrestors on the substation transformer, at each overhead switching device, at each transition from overhead to underground cable, and at the last transformer in the underground string. Lightning protection needs to be custom designed for the particular isokeraunic level and grounding. What we have included in our estimates, we believe, is adequate for the scope of this study.

13. Power quality meters for each row of turbines could be a helpful maintenance/project performance evaluation device. These meters would also require RTUs.

We proposed a recloser device at the point where a row of turbines is connected to the overhead line. The recloser serves as a protective device to detect and open for faults downstream (on the windmill side), as a remote-controlled threephase switch, and as a point to gather data, such as metering and status (i.e., open or closed). We included an RTU at these points to provide data and control back to the master station. 


\section{GLOSSARY}

The following definitions are for a general understanding of the terms used in this report. A technical user should refer to the scientific definitions.

ACSR: Aluminum conductor steel reinforced. This refers to a wire cable used for overhead lines that comprises one or more strands of steel wire surrounded by strands of aluminum wire.

Active Power or Power: The product of voltage and the in-phase component of alternating current. The active power does work and is measured in watts.

Ampere or Amp (A): Unit of current. One volt across one ohm of resistance gives a current flow of one ampere.

Capacitor: A device that stores electrical charge and provides leading VARs in a power circuit. Refer to "Reactive Power" below.

Circuit Breaker: A device designed to open and close an electrical circuit.

Current: The flow of electric charge.

Hertz (Hz): Unit for frequency in cycles per second.

kcmil: Thousand circular mils, used to define the size of a wire.

Kilovolts (kV): 1,000 volts.

Kilowatts (kW): 1,000 watts.

kVA: 1,000 volt-amps.

Load Tap Changer (LTC): A device on a transformer that adjusts the output voltage.

Megawatts (MW): 1,000,000 watts.

MVA: $1,000,000$ volt-amps.

Power: Refers to active power.

Ohm: Unit of electrical resistance. One volt across one ohm of resistance gives a current flow of one ampere. 
Resistance: Opposition that a conductor or electrical device offers to current flow, measured in ohms.

Transformer: An electrical device with two or more magnetically coupled windings that connects power system elements of different voltages.

Reactive Power: The product of voltage and the out-of-phase component of alternating current. A current wave ahead of or leading the voltage wave provides leading reactive power (or leading VARs). A current wave behind or lagging the voltage wave provides lagging reactive power (or lagging VARs). Line losses are lagging reactive power. A capacitor provides leading reactive power that compensates for the line losses.

RTU: For Remote Terminal Unit. An electronic device that connects to the communication media (fiber optic in this case) and used to communicate data to and from a location.

SCADA: For System Control and Data Acquisition. Equipment used to send data from a remote location to a central location and to communicate control commands from a central location to remote devices.

VAR: Unit of reactive power. Var is Volt-Amp-Reactive.

Volt-Amp (VA): The product of voltage and current, used for rating generators and transformers.

Volt (V): Unit of electromotive force. One volt across one ohm of resistance gives a current flow of one ampere. 
Figure 1

Figure 2

Figure 3

Figure 4

Figure 5

Figure 6

Figure 7

Figure 8

Figure 9

Figure 10

Figure 11

Figure 12

Figure 13

Figure 14

Figure 15
Great Plains Region

10-m Wind Rose Graph

750-kW 15-kV Plan

750-kW 25-kV Plan

2.5-MW 25-kV Plan

2.5-MW 35-kV Plan

5-MW 25/35-kV Plan

10-MW 25/35-kV Plan

Collector System One-Line Diagram

Communications System One-Line Diagram

Relative Foundation Geometry

Windmill Size and Foundation Comparison

Foundation Cost

Rock Foundation

Cost Comparison: Pad and Pier vs. Rock Anchor 
Figure 1

\section{Great Plains Region}

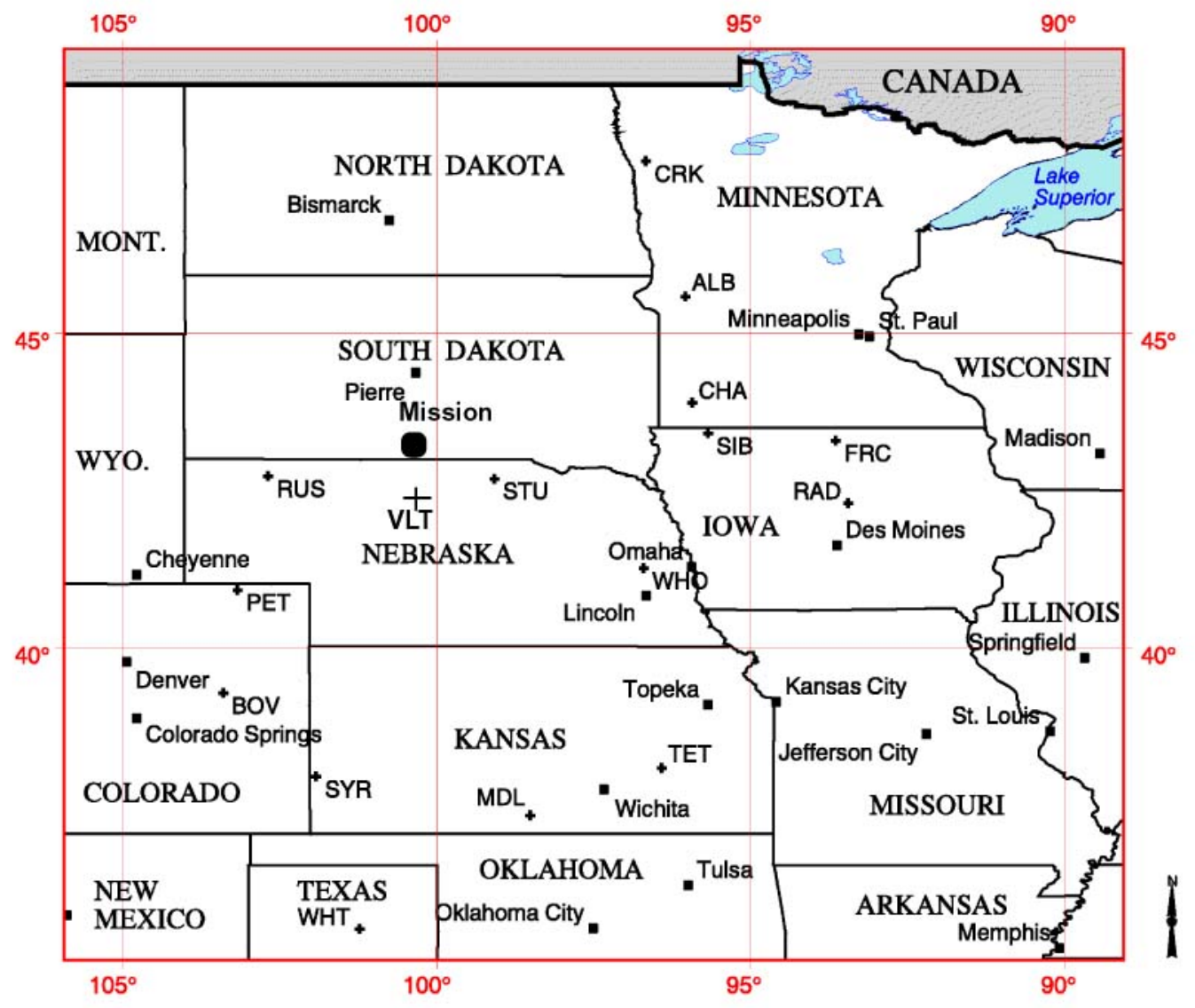

+ Wind Energy Monitoring Site

- City 
Figure 2

10-m Wind Rose Graph

1996-97

\section{Valentine, Nebraska}

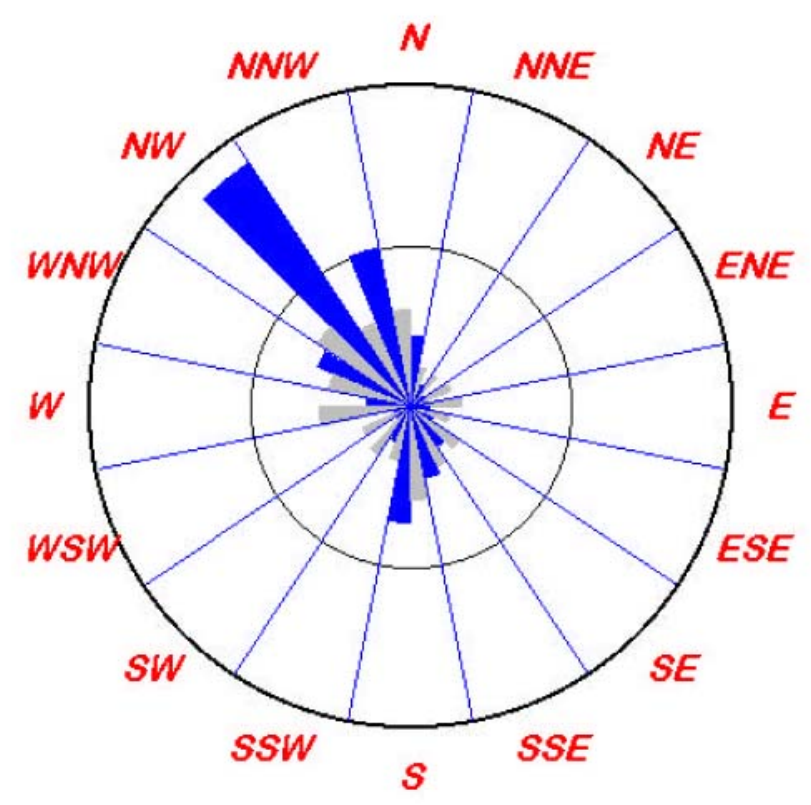

Pierre, South Dakota

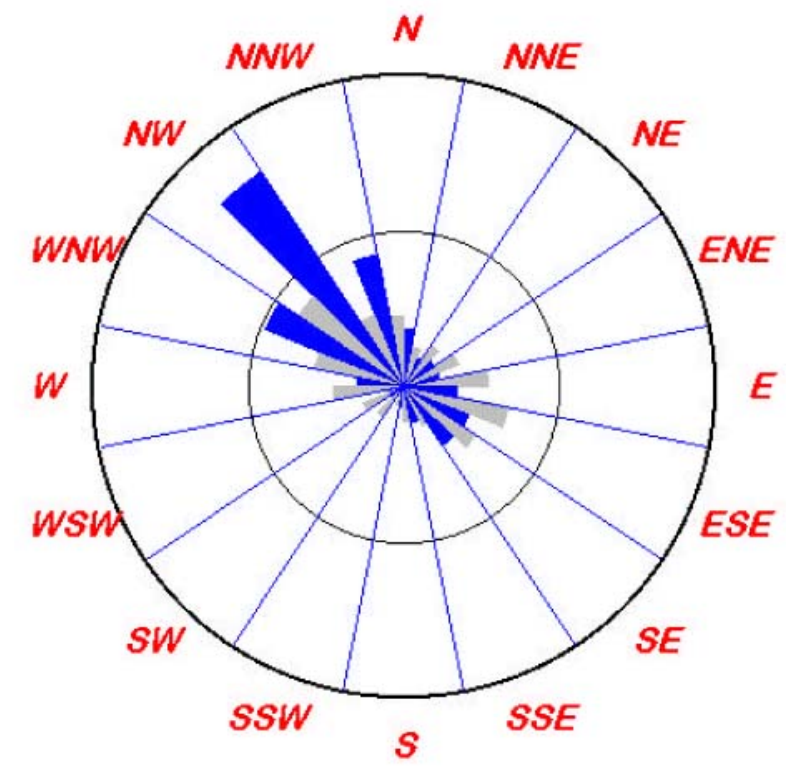

Percent of Total Wind Energy $\left(\mathrm{Wh} / \mathrm{m}^{2}\right)$ :

Percent of Total Time:

Circle Center: 0.0\%

Inner Circle: $15.0 \%$

Outer Circle: $30.0 \%$ 


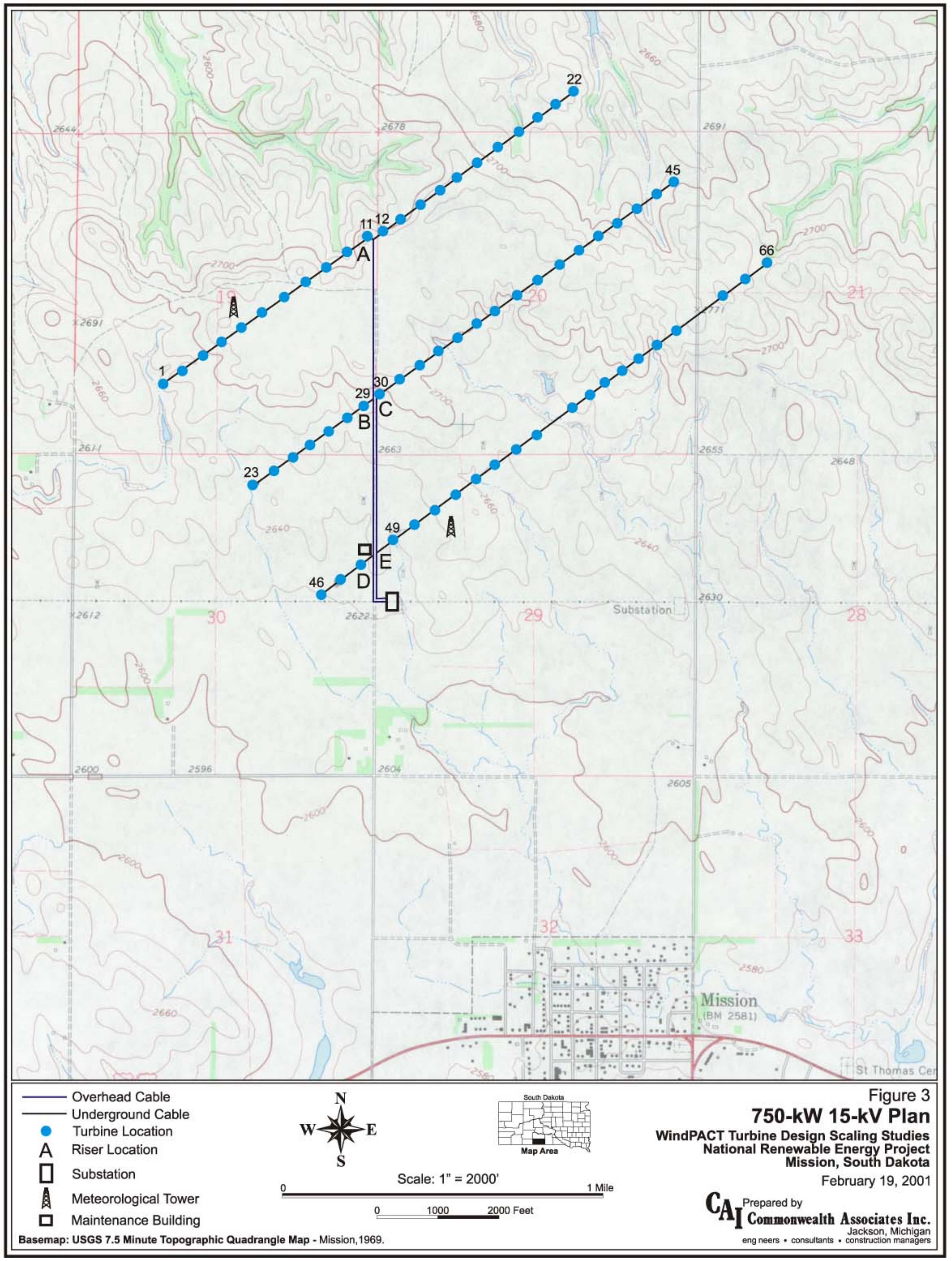




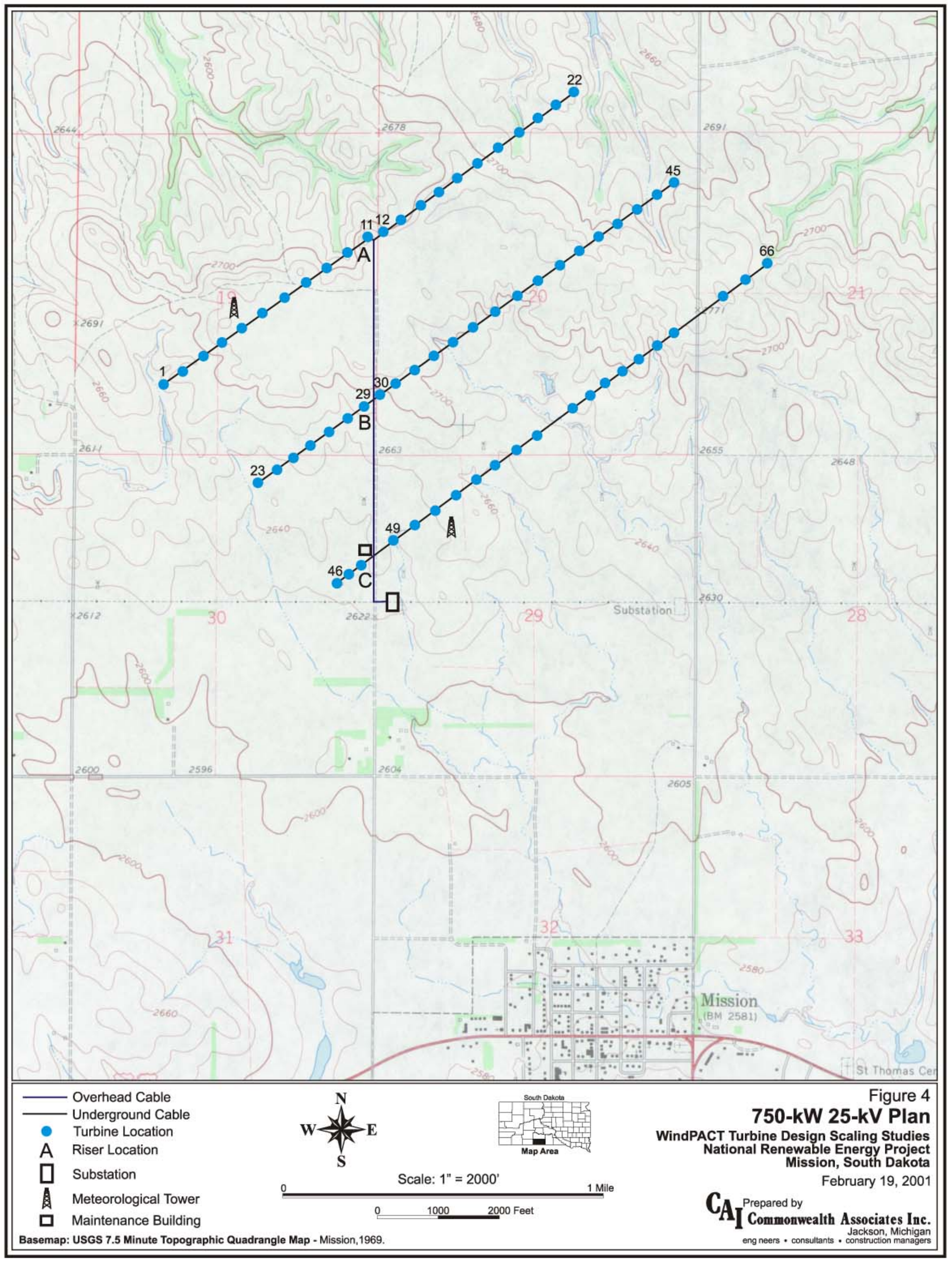




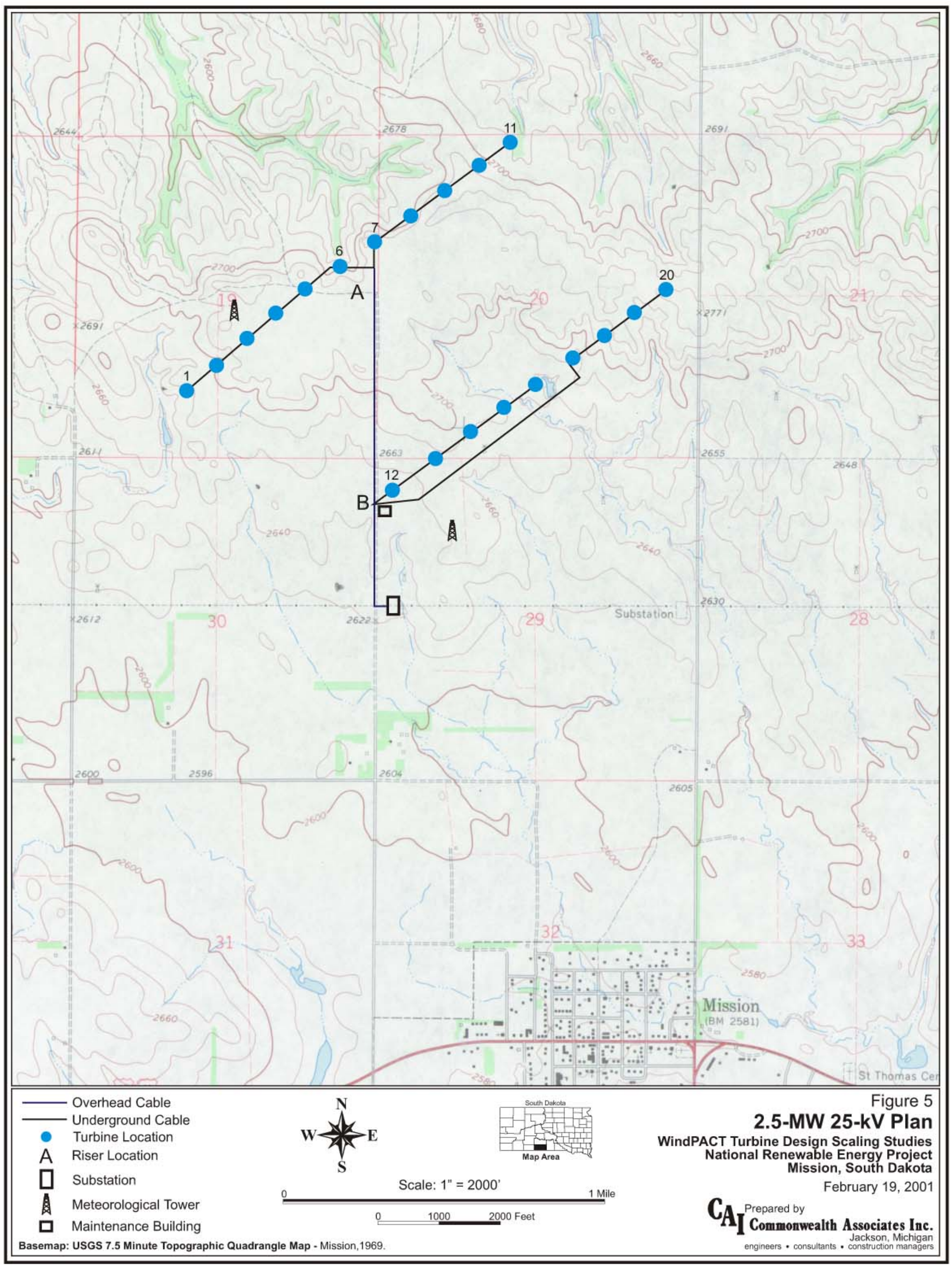




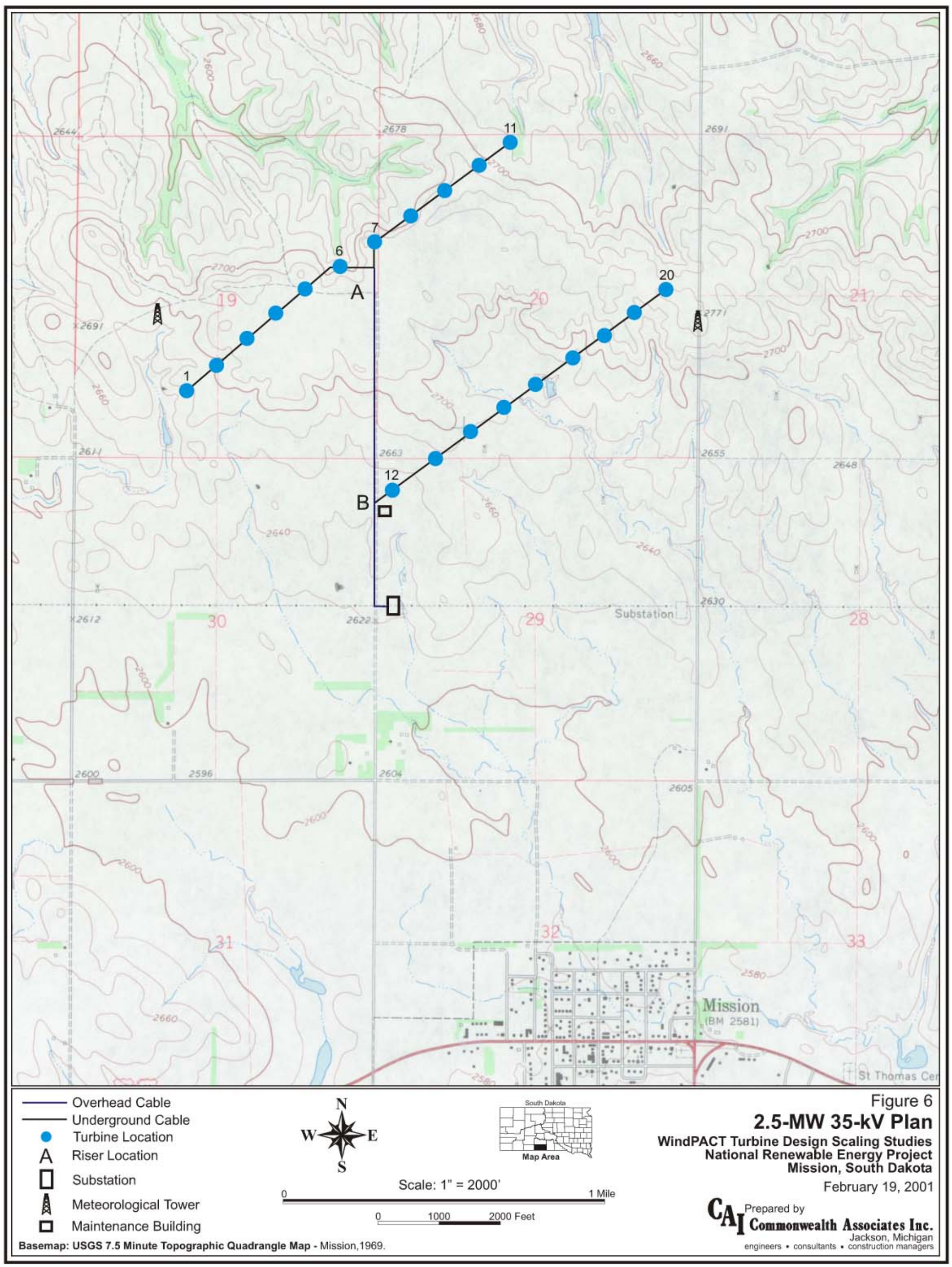




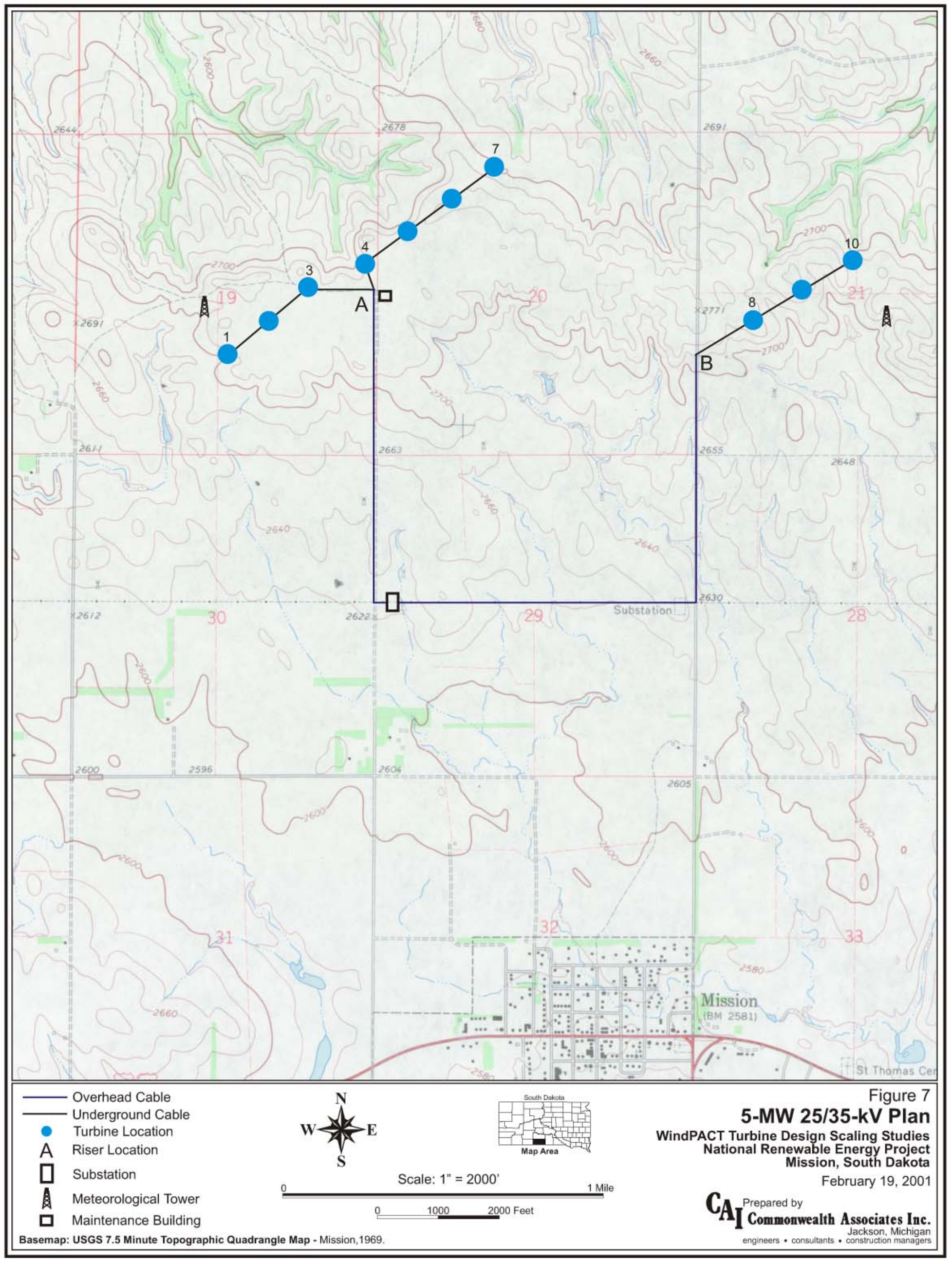




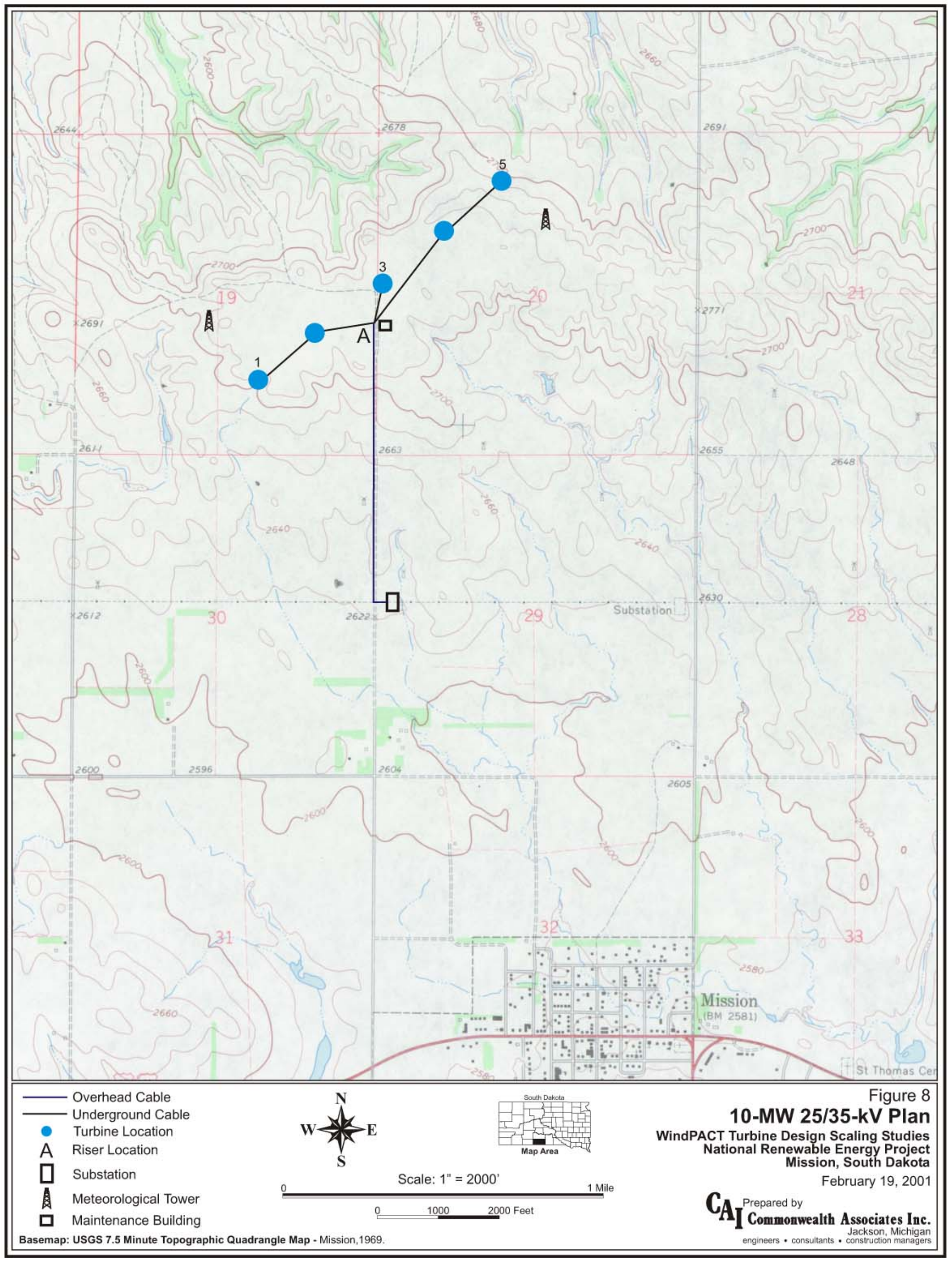




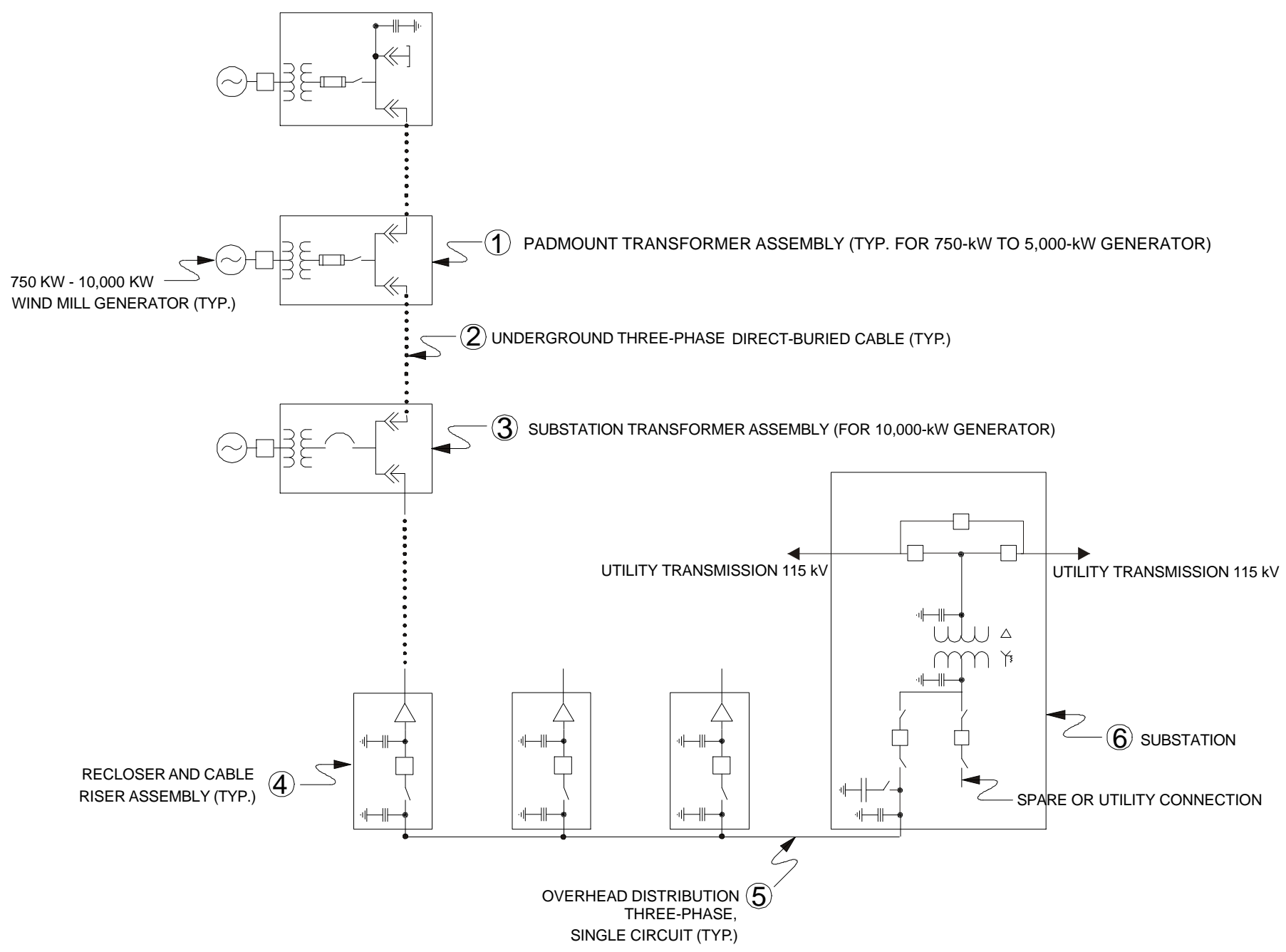

\begin{tabular}{|c|c|}
\hline \multicolumn{2}{|c|}{ Symbol Key } \\
\hline 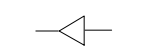 & Pothead \\
\hline- & Circuit Breaker \\
\hline$-\smile \square-$ & Circuit Breaker \\
\hline$-\square \square-$ & Fuse \\
\hline$\|\mapsto\| \bullet$ & Surge Arrestor \\
\hline$\oslash$ & Generator \\
\hline$\beta \xi$ & Transformer \\
\hline$-\ll$ & Disconnect Device \\
\hline$\| \mapsto \nVdash^{\prime}-$ & Capacitor \\
\hline .......... & Underground Cable \\
\hline
\end{tabular}

WindPACT Turbine Design Scaling Studies National Renewable Energy Project Mission, South Dakota FIGURE 9 COLLECTOR SYSTEM ONE-LINE DIAGRAM

\begin{tabular}{|c|c|c|}
\hline \multirow{2}{*}{$\begin{array}{l}\text { CAI Commontwealth Associates Inc. } \\
\text { Jackson, Michigan } \\
\text { engineers • consultants } \begin{array}{c}\text { construction managers } \\
\text { Preped by }\end{array}\end{array}$} & $\begin{array}{c}\text { DATE } \\
02 / 19 / 01 \\
\end{array}$ & $\begin{array}{c}\text { PROJECTTUMBER } \\
228001\end{array}$ \\
\hline & $\begin{array}{c}\begin{array}{c}\text { BY } \\
\text { RMC }\end{array} \\
\end{array}$ & $\begin{array}{l}\text { PROJECTMANAGERER } \\
\text { DA SHAFFR }\end{array}$ \\
\hline FILE: M:PROJINRELL228001 1 PDF Figures|Figure 9.pd & \multicolumn{2}{|c|}{ DWGNO: 228001-OL } \\
\hline
\end{tabular}




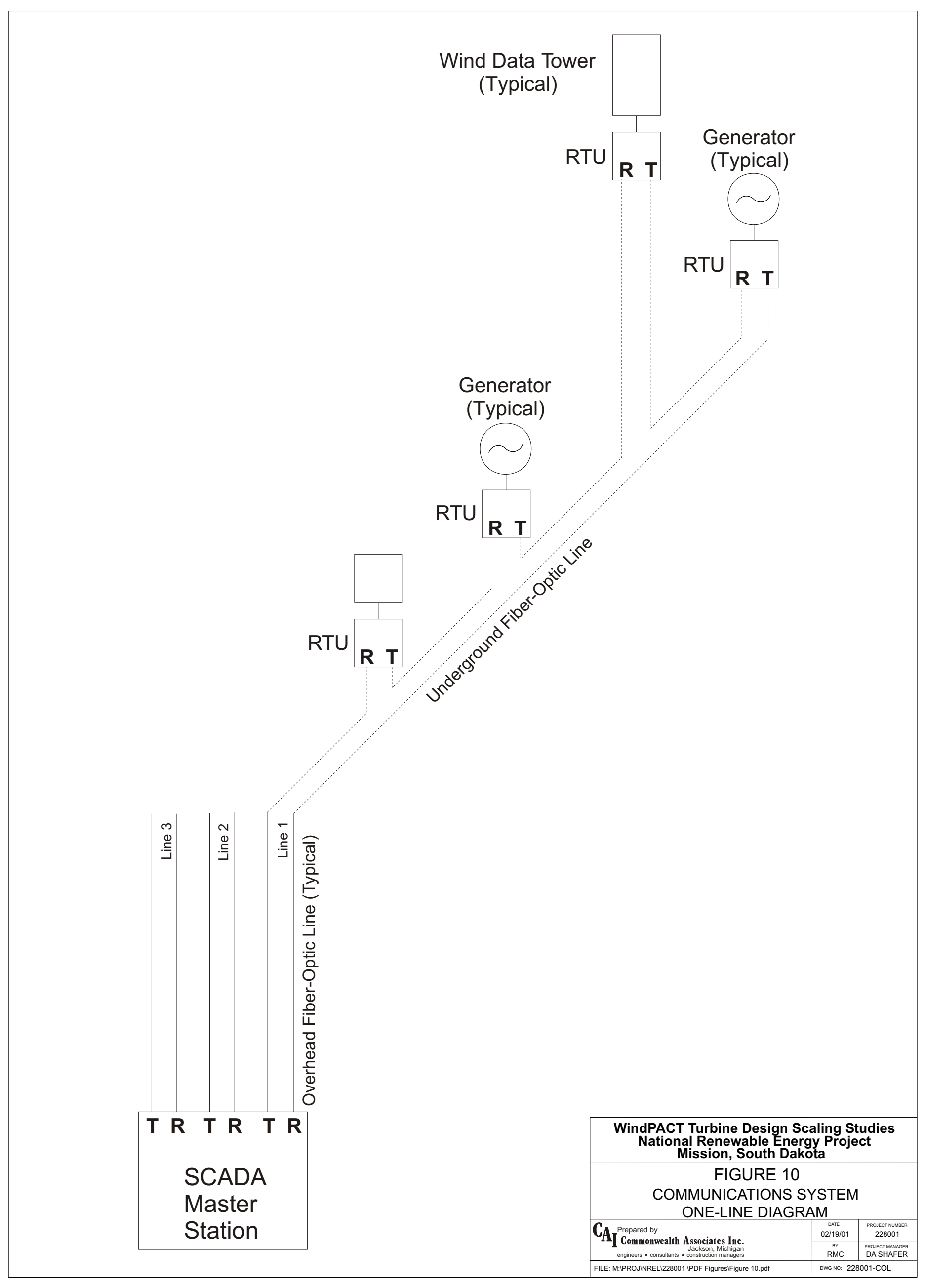


Figure 11

Relative Foundation Geometry

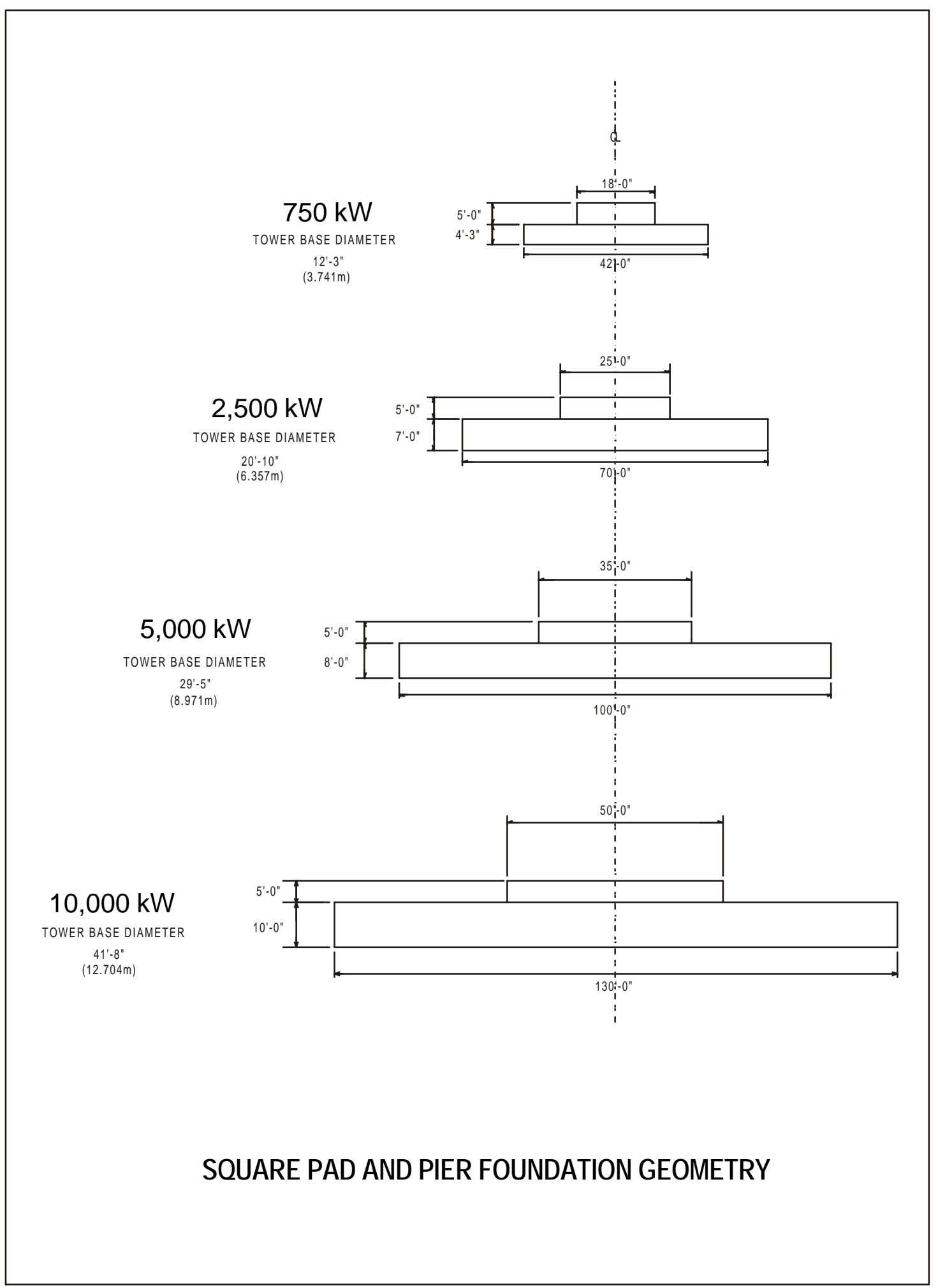


Figure 12

Windmill Size and Foundation Comparison

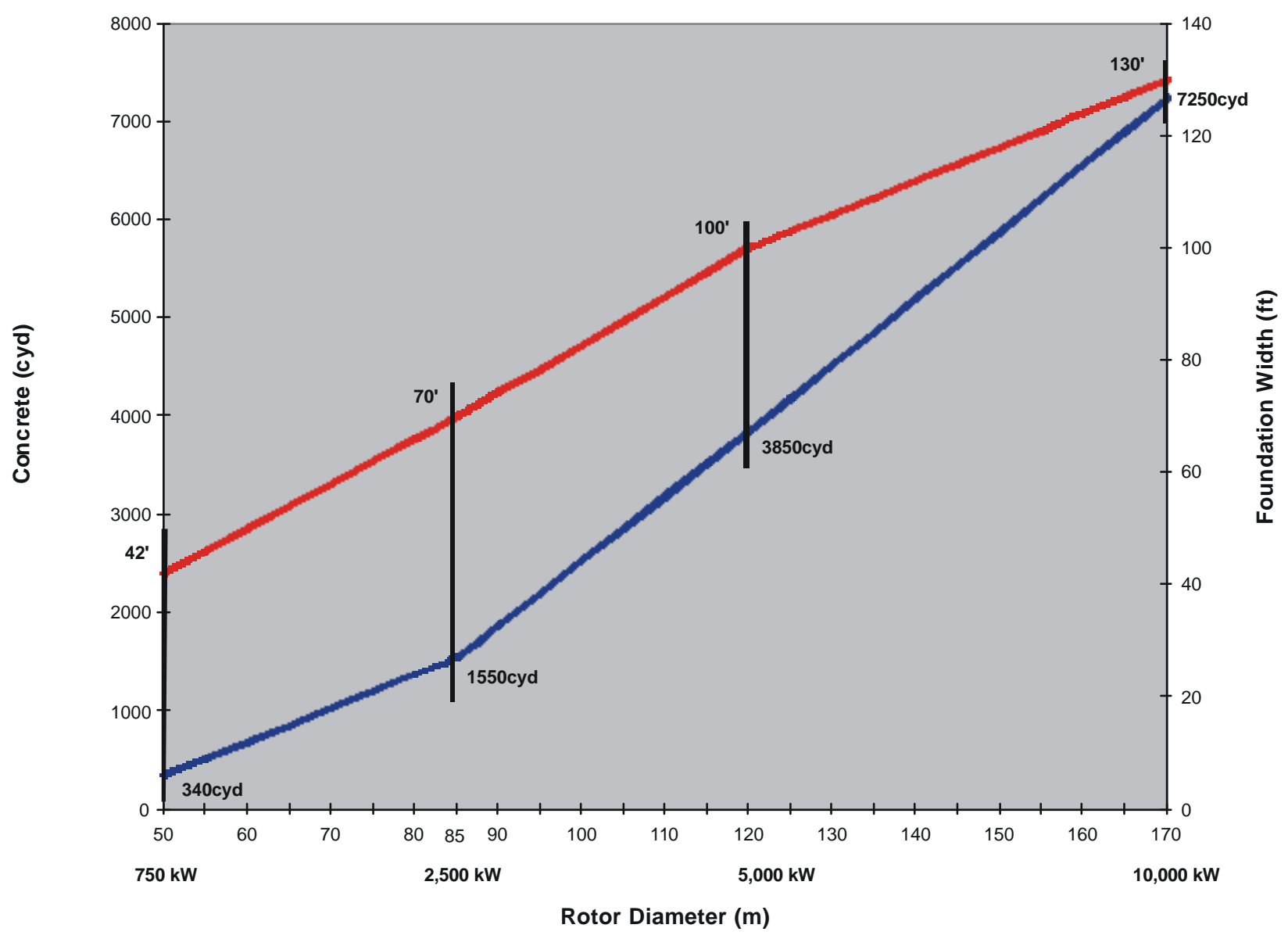

Concrete (cyd)

Width (ft) 
Figure 13

Foundation Cost

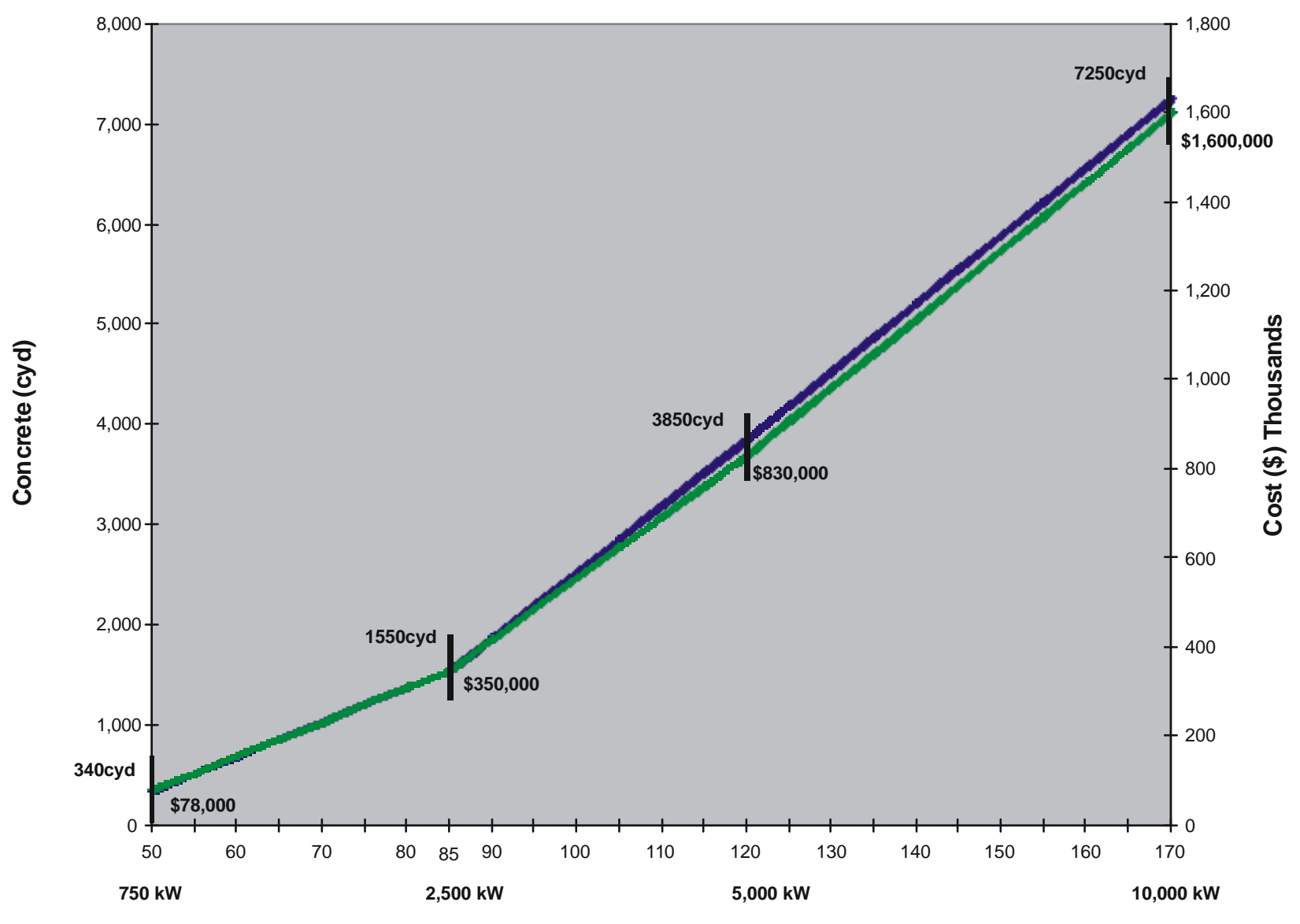

Rotor Diameter (m) 
Figure 14

\section{Rock Foundation}

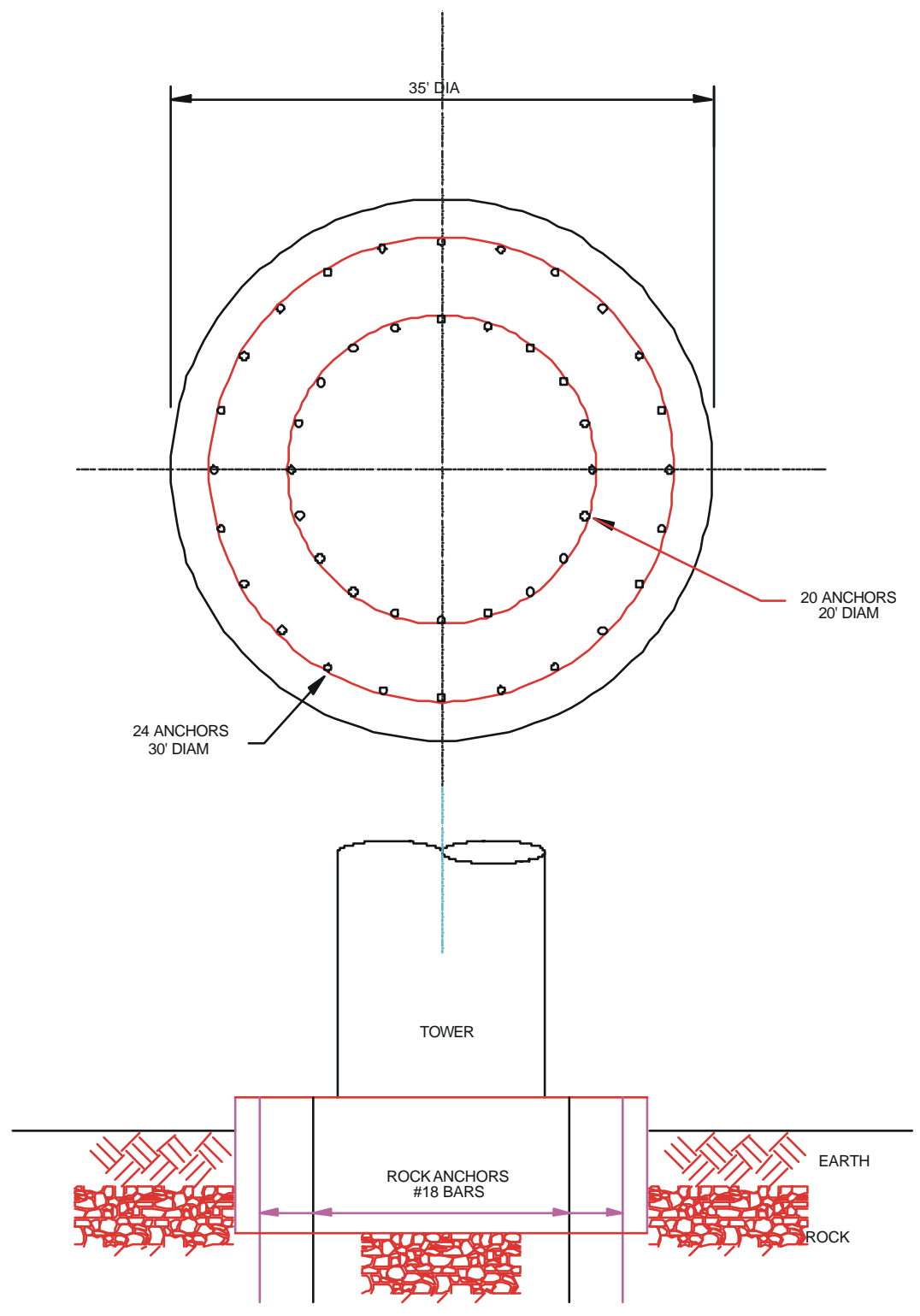

PROPOSED FOCK AVCHOF FOUNDATION

FOR $750 \mathrm{~kW}$ (50 m ROTOR DIAMETER WINDMILL) 
Figure 15

Cost Comparison: Pad and Pier vs. Rock Anchor

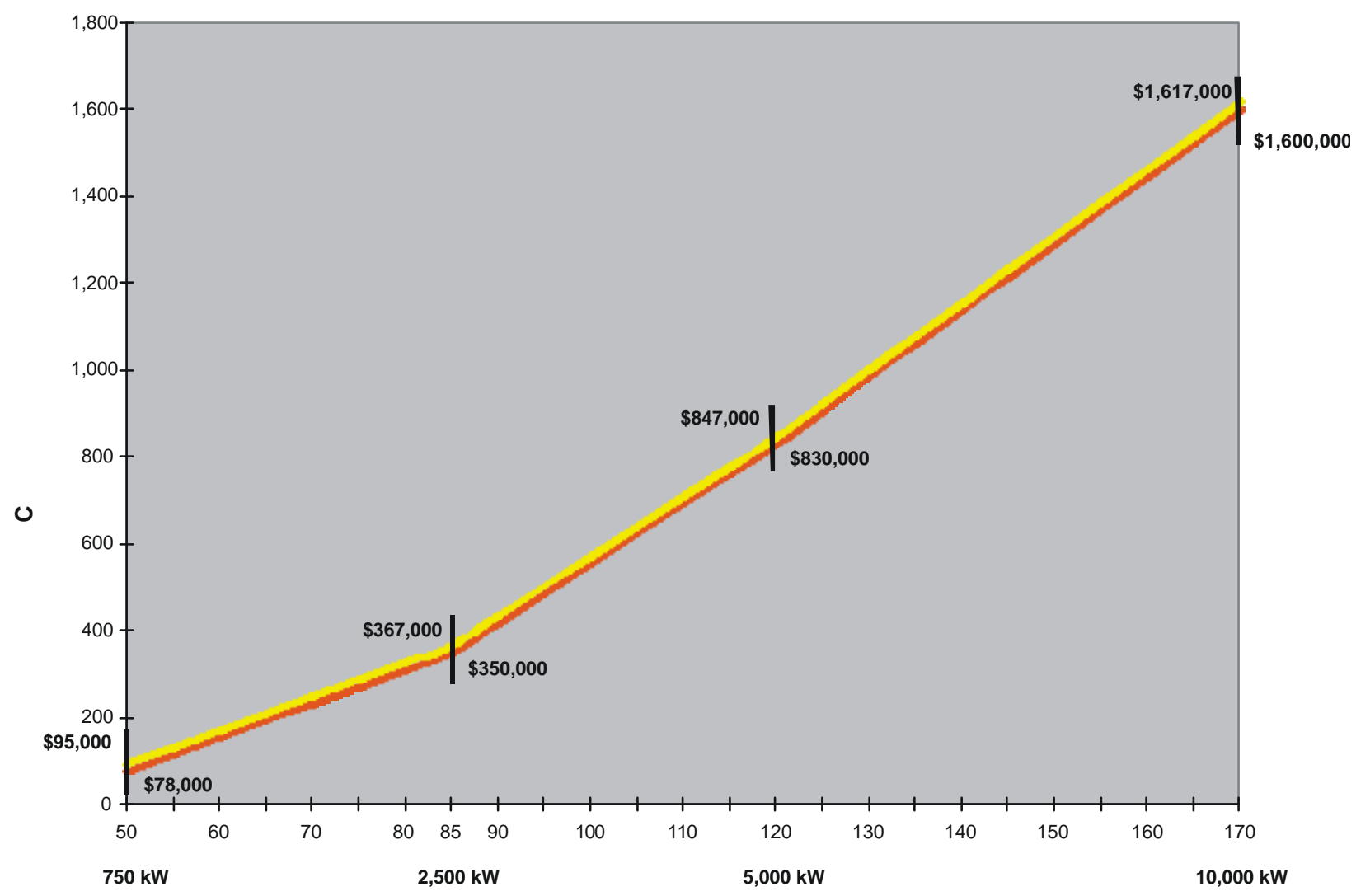

Rotor Diameter (m) 
Table A1 Turbine Parameters and Assumptions

Table A2 Three-Phase Transformer Data Estimated Values

Table A3.1 Estimated Cable Size for Radially Connected Wind Turbines

Table A3.2 Typical 15-kV Underground Primary Distribution Cable

Table A3.3 Typical 25-kV Underground Primary Distribution Cable

Table A3.4 Typical 35-kV Underground Primary Distribution Cable

Table A3.5 Overhead Distribution Cable 


\section{APPENDIX A \\ ENGINEERING ASSUMPTIONS \\ AND DATA}

\section{WIND TURBINE PARAMETERS AND ASSUMPTIONS}

Table A1 shows the parameters and assumptions used in this study to model the wind turbines.

\section{TRANSFORMER ASSUMPTIONS}

Table A2 provides the parameters for the generator step-up transformers. Assumptions are listed below:

1. Standard three-phase padmount transformers were used for the $750-\mathrm{kW}(1,000 \mathrm{kVA}$, $480 \mathrm{~V}), 2,500-\mathrm{kW}(3,000 \mathrm{kVA}, 690 \mathrm{~V})$, and 5,000-kW (7,500 kVA, 2,400V) plans. For this study, we assumed grounded-wye low-side and delta high-side connections. The primary $(13.8 \mathrm{kV}, 24.9 \mathrm{kV}$, or $34.5 \mathrm{kV})$ would be dead front design. The manufacturer provides a "loop" design, which allows the primary cable to be "looped" from one transformer to the next, as we are proposing. We have also included a high-voltage switch that would allow one wind turbine to be disconnected while still keeping all others in the string in service. The transformer would be protected by the manufacturer's standard fusing arrangement.

2. The manufacturers standardize on two sizes of transformer and cable terminations: 200 amps and 600 amps. For this study, we assumed that we would purchase identical transformers with the high-side connections and cable terminations rated for $600 \mathrm{amps}$.

3. Since 7,500 kVA is the largest standard padmount transformer capacity, a 10/12.5-MVA substation transformer (grounded-wye low-side, delta high-side, 4,160 V) was used for the $10,000 \mathrm{~kW}$ plan. While not considered a dead front design, cabinets can be installed on this transformer to cover otherwise exposed electrical connections and to allow both low- and high-side connections to be fed underground. To protect the transformer, padmounted switchgear with a vacuum interrupter (circuit breaker) was connected to the high side. This will allow the primary cable to be "looped" from one transformer to the next where necessary.

\section{CABLE ASSUMPTIONS}

Parameters for the underground cables are provided in Table A3. Assumptions are listed below:

1. We assumed all underground collector lines were constructed using standard 1/0, 4/0, $500-\mathrm{kcmil}$, or $750-\mathrm{kcmil}$ aluminum underground cables. Three individual cables were used to create the three-phase circuit rather than a manufactured single three-conductor

cable. The advantages of using three separate conductors are lower cost and longer runs 
between splices. A single three-phase $1 / 0$ aluminum cable is rated approximately 216 amps. At $13.8 \mathrm{kV}$, each $750-\mathrm{kW}$ wind turbine produces 32.3 amps. Thus, six wind turbines can be carried on a $1 / 0$ cable before exceeding its 216-amp rating. Similarly, nine turbines can be carried on $4 / 0$ cable, 15 on $500-\mathrm{kcmil}$ cable, and up to 18 on 750 kcmil aluminum cable. Table A3.1 summarizes cable ampacities.

2. Where the rating of $1 / 0$ aluminum conductor would have been exceeded, larger conductor was used. An alternative would have been to parallel two or more sets of $1 / 0$ cable. For this analysis, the choice was to use multiple conductor sizes in an attempt to optimize the cable size based on costs and losses.

\section{OVERHEAD LINE ASSUMPTIONS}

Table A3.5 provides parameters for the overhead cables. Assumptions are listed below:

1. The overhead line is assumed to be conventional utility distribution design using wood poles and cross arms. At $13.8 \mathrm{kV}, 50 \mathrm{MW}$ translates to approximately 2,091 amps. This is more than is typically served by a single $13.8-\mathrm{kV}$ feeder. One standard equipment size is 1,200 amps. Therefore, we assumed that a $13.8-\mathrm{kV}$ design would require two main collector lines and substation feeder breakers. We assumed that both circuits would be constructed on a single pole. At $24.9 \mathrm{kV}, 50 \mathrm{MW}$ translates to approximately 1,159 amps. Therefore, 24.9- and $34.5-\mathrm{kV}$ designs require only single-circuit overhead construction.

2. For six of the eight plans, we chose $795 \mathrm{kcmil}$ ACSR conductors for the overhead line. This conductor is nominally rated for 907 amps. However, this rating is based on high ambient temperatures and calm conditions. Since we are expecting to hit peak generating capabilities during windy conditions, we expect that 795 ACSR will be suitable. Similar to our selection for underground cable, we attempted to optimize the conductor size for overhead installation. For the 5,000-kW layout, two overhead lines are needed because the wind turbines are placed in two separate groups. For this layout, 795-kcmil ACSR was used for the $24.9-\mathrm{kV}$ plan, and 4/0 ACSR was used for the $34.5-\mathrm{kV}$ plan.

3. Our cost estimates are based on installing a neutral on top of the pole. This wire serves a dual purpose as a neutral and shield wire. The purpose of a shield wire is to provide lightning protection to the circuit. A neutral is needed for connecting line-to-neutral single-phase transformers. Because the windmills are three-phase, we could design the collector line without a neutral. However, we have provided a neutral, as this is common practice and could be used by the utility for serving single-phase loads.

\section{SUBSTATION ASSUMPTIONS}

1. For this study, we used a packaged 30/40/50-MVA substation, a Power Delivery System (PDS), that is manufactured by Waukesha Electric Systems. The packaged substation includes a $115 / 13.8-\mathrm{kV}$ transformer and three $13.8-\mathrm{kV}$ and two $24.9-$ or $34.5-\mathrm{kV}$ feeder

circuit breakers. The PDS includes all of the above assembled, factory-tested, and 
shipped to the customer's site. The customer provides a foundation, grounding, oil containment, and fencing, and connects the $115-\mathrm{kV}$ and low-side feeders to complete the substation.

2. We assumed a three-breaker $115-\mathrm{kV}$ ring bus would be used to connect to the existing $115-\mathrm{kV}$ line. 
Table A1

WindPACT Turbine Design Scaling Study

\section{Turbine Parameters and Assumptions}

\begin{tabular}{|c|c|c|c|c|c|c|c|}
\hline $\begin{array}{c}\text { Plant Size } \\
\text { kW }\end{array}$ & $\begin{array}{c}\text { Rotor } \\
\text { Diameter }\end{array}$ & $\begin{array}{c}\text { Min. Distance }{ }^{1} \\
\text { Between Turbines }\end{array}$ & $\begin{array}{l}\text { Min. Distance }{ }^{1} \\
\text { Between Rows }\end{array}$ & $\begin{array}{l}\text { Machir } \\
\text { kVA }\end{array}$ & $\begin{array}{c}\text { Base }^{2} \\
k V\end{array}$ & $\begin{array}{l}\text { SC Model } \\
\text { X"d }\end{array}$ & $\begin{array}{c}\text { Total }^{3} \\
\text { Turbines }^{2}\end{array}$ \\
\hline 750 & $50 \mathrm{~m}(164 \mathrm{ft})$ & $115 \mathrm{~m}(378 \mathrm{ft})$ & $600 \mathrm{~m}(1969 \mathrm{ft})$ & 833 & 0.48 & 0.107 & 66 \\
\hline 2500 & $85 \mathrm{~m}(279 \mathrm{ft})$ & $196 \mathrm{~m}(642 \mathrm{ft})$ & $1020 \mathrm{~m}(3347 \mathrm{ft})$ & 2,778 & 0.69 & 0.107 & 25 \\
\hline 5000 & $120 \mathrm{~m}(394 \mathrm{ft})$ & $276 \mathrm{~m}(906 \mathrm{ft})$ & $1440 \mathrm{~m}(4725 \mathrm{ft})$ & 5,556 & 2.40 & 0.107 & 10 \\
\hline 10000 & $170 \mathrm{~m}(558 \mathrm{ft})$ & $391 \mathrm{~m}(1283 \mathrm{ft})$ & $2040 \mathrm{~m}(6693 \mathrm{ft})$ & 11,111 & 4.16 & 0.107 & 5 \\
\hline
\end{tabular}

$\begin{array}{cc}\begin{array}{c}\text { Plant Size } \\ \text { kW }\end{array} & \begin{array}{c}\text { Total } \\ \text { Mass }^{4}\end{array} \\ 750 & 104,927 \mathrm{~kg} \\ 2500 & 466,061 \mathrm{~kg} \\ 5000 & 1,237,715 \mathrm{~kg} \\ 10000 & 3,338,895 \mathrm{~kg}\end{array}$

Total
Overturning Moment
$38,718 \mathrm{kN}-\mathrm{m}$
$190,185 \mathrm{kN}-\mathrm{m}$
$535,070 \mathrm{kN}-\mathrm{m}$
$1,521,106 \mathrm{kN}-\mathrm{m}$

Tower Base
Diameter

$3.7 \mathrm{~m}$
$6.4 \mathrm{~m}$
$9.0 \mathrm{~m}$
$12.7 \mathrm{~m}$

Option 2

Plant Size

Crane

kW

$\operatorname{Pad}(\mathbf{s})$ Size

Maintenance

Building Size

750

2500

5000

10000

$\begin{array}{cc}243 \mathrm{~m}^{2} & 40^{\prime} \mathrm{W} \times 60^{\prime} \mathrm{L} \times 16^{\prime} \mathrm{H} \\ 1000 \mathrm{~m}^{2} & 40^{\prime} \mathrm{W} \times 60^{\prime} \mathrm{L} \times 16^{\prime} \mathrm{H} \\ 2400 \mathrm{~m}^{2} & 40^{\prime} \mathrm{W} \times 60^{\prime} \mathrm{L} \times 16^{\prime} \mathrm{H} \\ 4000 \mathrm{~m}^{2} & 40^{\prime} \mathrm{W} \times 60^{\prime} \mathrm{L} \times 16^{\prime} \mathrm{H}\end{array}$

Access Road Width

\begin{tabular}{|c|c|}
\hline $8 \mathrm{~m}(26 \mathrm{ft})$ & One RTU / Recloser, \\
\hline & wer, \\
\hline $\begin{array}{l}0 \mathrm{~m} \\
0 \mathrm{~m}\end{array}$ & $\begin{array}{l}\text { and Turbin } \\
\text { four Plant }\end{array}$ \\
\hline
\end{tabular}

NOTES:

1. Based on a turbine spacing of 2.3 rotor diameters by a row spacing of 12 rotor diameters.

2 Assumed rated power factor of $90 \%$.

3. Number of Turbines needed for $50 \mathrm{MW}$.

4. Corrected values from those presented at the November $2 \& 3$ workshop. 


\section{Table A2}

\section{WindPACT Turbine Design Scaling Studies}

Three-Phase Transformer Data

\section{Estimated Values}

\section{Pad-mount Transformers}

\begin{tabular}{|c|c|c|c|c|c|c|c|c|}
\hline \multirow{2}{*}{$\begin{array}{c}\text { Gen. Rating } \\
\text { kW }\end{array}$} & \multicolumn{3}{|c|}{ Voltage } & \multicolumn{2}{|r|}{ Fluid } & \multirow{2}{*}{$\begin{array}{l}\text { Approx. Weight } \\
\text { lbs. }\end{array}$} & \multicolumn{2}{|c|}{ Pad Dimensions ${ }^{2}$} \\
\hline & kVA $^{1}$ & kV & $\mathbf{Z} \%$ & $\mathbf{X} / \mathbf{R}$ & Gal. & & $\mathbf{x}$ & $\mathbf{Y}$ \\
\hline 750 & 1000 & $0.48 / 13.8$ & 5.75 & 9 & 480 & 10100 & 93 & 63 \\
\hline 750 & 1000 & $0.48 / 24.9$ & 5.75 & 9 & 480 & 10100 & 93 & 63 \\
\hline 2500 & 3000 & $0.69 / 13.8$ & 5.5 & 9 & 760 & 18850 & 76 & 103 \\
\hline 2500 & 3000 & $0.69 / 24.9$ & 5.5 & 9 & 760 & 18850 & 76 & 103 \\
\hline 2500 & 3000 & $0.69 / 34.5$ & 7.0 & 9 & 760 & 18850 & 76 & 103 \\
\hline 5000 & 7500 & $2.40 / 24.9$ & 6.50 & 9 & 1580 & 41900 & 100 & 126 \\
\hline 5000 & 7500 & $2.40 / 34.5$ & 6.50 & 9 & 1580 & 41900 & 100 & 126 \\
\hline \multicolumn{9}{|c|}{ Substation Transformers } \\
\hline 10000 & 12500 & $4.16 / 24.9$ & 6.50 & 9 & 1600 & 46500 & 157 & 153 \\
\hline 10000 & 12500 & $4.16 / 34.5$ & 7.00 & 9 & 1600 & 46500 & 157 & 153 \\
\hline
\end{tabular}

\section{NOTES}

1. OA Self-cooled rated, $65^{\circ} \mathrm{C}$ temperature rise.

2. Pad Dimensions measured in inches. 
Table A3.1

\section{WindPACT Turbine Design Scaling Studies}

\section{Estimated Cable Size for Radially Connected Wind Turbines}

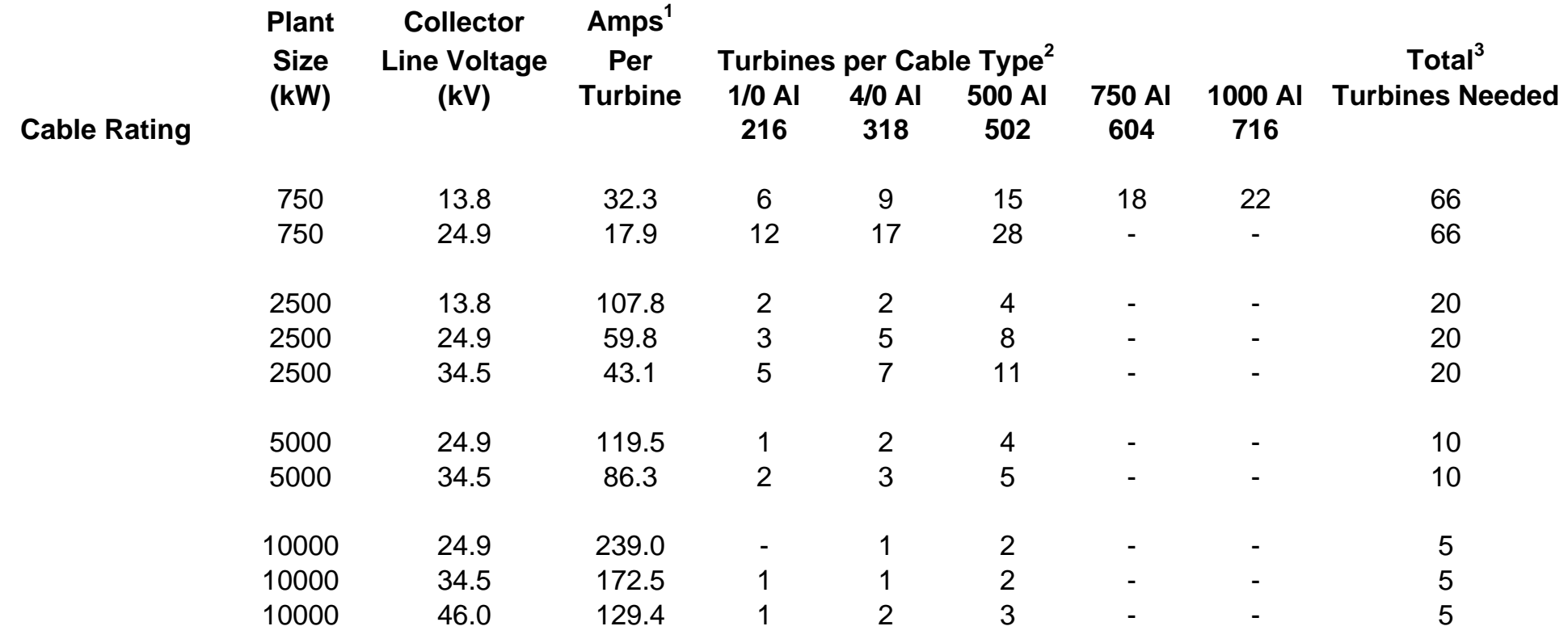

\section{NOTES}

1. The amps per turbine is based on a net turbine power factor of $97 \%$.

2. The number of turbines radially connected based on cable capacity.

The cable rating is based on a load factor rating of $75 \%$.

3. The number of wind turbines needed to produce $50 \mathrm{MW}$. 
Table A3.2

\section{WindPACT Turbine Design Scaling Studies}

\section{Typical 15 kV Underground Primary Distribution Cable \\ Three 1/C Aluminum Conductor \\ One-Third Copper Concentric Neutral with Jacket \\ 220 Mil Insulation (133\%)}

$\begin{array}{lccccc}\begin{array}{c}\text { Size } \\ \text { (AWG/ } \\ \text { kcmil) }\end{array} & \begin{array}{c}\text { No. } \\ \text { Of } \\ \text { Str. }\end{array} & \begin{array}{c}\text { Overall } \\ \text { Cable O.D. } \\ \text { (Inches) }\end{array} & \begin{array}{c}\text { Cable } \\ \text { Weight } \\ \text { (Lbs/Kft) }\end{array} & \begin{array}{c}\text { Capacitance } \\ \text { (pf/ft.) }\end{array} & \begin{array}{c}\text { Amps per Circu } \\ \text { Direct Buried }\end{array} \\ \text { 1 Circuit } & \text { 2 } \\ 1 / 0 & 19 & 1.19 & 661 & 66 & 216 \\ 4 / 0 & 19 & 1.34 & 933 & 84 & 318 \\ 500 & 37 & 1.74 & 1742 & 115 & 502 \\ 750 & 61 & 1.98 & 2380 & 136 & 604 \\ 1000 & 61 & 2.16 & 2983 & 152 & 716 \\ \\ \end{array}$


Table A3.3

WindPACT Turbine Design Scaling Studies

\section{Typical 25 kV Underground Primary Distribution Cable \\ Three 1/C Aluminum Conductor \\ One-Third Copper Concentric Neutral with Jacket 320 Mil Insulation (133\%)}

\begin{tabular}{|c|c|c|c|c|c|c|}
\hline \multirow{2}{*}{$\begin{array}{c}\text { Size } \\
\text { (AWG/ } \\
\text { kcmil) }\end{array}$} & \multirow{2}{*}{$\begin{array}{l}\text { No. } \\
\text { Of } \\
\text { Str. }\end{array}$} & \multirow{2}{*}{$\begin{array}{c}\text { Overall } \\
\text { Cable O.D. } \\
\text { (Inches) }\end{array}$} & \multirow{2}{*}{$\begin{array}{c}\text { Cable } \\
\text { Weight } \\
\text { (Lbs/Kft) }\end{array}$} & \multirow{2}{*}{$\begin{array}{c}\text { Capacitance } \\
\text { (pf/ft.) }\end{array}$} & \multicolumn{2}{|c|}{$\begin{array}{l}\text { Amps per Circuit } \\
\text { Direct Buried }\end{array}$} \\
\hline & & & & & 1 Circuit & 2 Circuit \\
\hline $1 / 0$ & 19 & 1.42 & 906 & 52 & 212 & 185 \\
\hline $4 / 0$ & 19 & 1.57 & 1200 & 64 & 312 & 269 \\
\hline 500 & 37 & 1.93 & 2025 & 86 & 490 & 416 \\
\hline 750 & 61 & 2.20 & 2722 & 101 & 596 & 500 \\
\hline 1000 & 61 & 2.35 & 3265 & 112 & 704 & 586 \\
\hline & \multicolumn{6}{|c|}{ NOTES } \\
\hline & \multicolumn{6}{|c|}{$\begin{array}{l}\text { 1. All values from IEEE Std } 835-1994 \text {, IEEE Standard Power Cable Ampacity Tables } \\
\text { 2. Conductor Temperature: } 90^{\circ} \mathrm{C} \\
\text { 3. Insulation: EPR } \\
\text { 4. Installation: Direct buried, triplexed cable } \\
\text { 5. Load Factor: } 75 \% \\
\text { 6. Earth Temperature: } 25^{\circ} \mathrm{C}\end{array}$} \\
\hline
\end{tabular}

Cable Impedance Data (ohms/1,000 ft.)

Positive/Negative

Zero Sequence

$0.212+j 0.051$

$0.107+\mathrm{j} 0.046$

$0.048+j 0.040$

$0.034+j 0.037$

$0.549+j 0.263$

$0.334+j 0.110$

$0.158+j 0.040$

$0.106+j 0.028$

$0.028+j 0.035$

\footnotetext{
1. All values from IEEE Std 835-1994, IEEE Standard Power Cable Ampacity Tables.

All values from IEE $90^{\circ} \mathrm{C}$

4. Installation: Direct buried, triplexed cable

6. Earth Temperature: $25^{\circ} \mathrm{C}$
} 
Table A3.4

\section{WindPACT Turbine Design Scaling Studies}

\section{Typical 35 kV Underground Primary Distribution Cable \\ Three 1/C Aluminum Conductor \\ One-Third Copper Concentric Neutral with Jacket \\ 420 Mil Insulation (133\%)}

\begin{tabular}{|c|c|c|c|c|c|c|c|c|}
\hline \multirow{2}{*}{$\begin{array}{c}\text { Size } \\
\text { (AWG/ } \\
\text { kcmil) }\end{array}$} & \multirow{2}{*}{$\begin{array}{l}\text { No. } \\
\text { Of } \\
\text { Str. }\end{array}$} & \multirow{2}{*}{$\begin{array}{c}\text { Overall } \\
\text { Cable O.D. } \\
\text { (Inches) }\end{array}$} & \multirow{2}{*}{$\begin{array}{c}\text { Cable } \\
\text { Weight } \\
\text { (Lbs/Kft) }\end{array}$} & \multirow{2}{*}{$\begin{array}{c}\text { Capacitance } \\
\text { (pf/ft.) }\end{array}$} & \multicolumn{2}{|c|}{$\begin{array}{l}\text { Amps per Circuit } \\
\text { Direct Buried }\end{array}$} & \multicolumn{2}{|c|}{$\begin{array}{c}\text { Cable Impedance Data } \\
\text { (ohms/1,000 ft.) }\end{array}$} \\
\hline & & & & & 1 Circuit & 2 Circuit & Positive/Negative & Zero Sequence \\
\hline $1 / 0$ & 19 & 1.65 & 1169 & 37 & 212 & 185 & $0.212+j 0.055$ & $0.545+j 0.267$ \\
\hline $4 / 0$ & 19 & 1.82 & 1564 & 45 & 312 & 269 & $0.107+j 0.049$ & $0.333+j 0.113$ \\
\hline 500 & 37 & 2.18 & 2446 & 60 & 490 & 416 & $0.047+j 0.042$ & $0.158+j 0.044$ \\
\hline 750 & 61 & 2.41 & 3126 & 69 & 596 & 500 & $0.034+j 0.039$ & $0.106+j 0.031$ \\
\hline 1000 & 61 & 2.56 & 3696 & 77 & 704 & 586 & $0.028+j 0.037$ & $0.080+j 0.026$ \\
\hline
\end{tabular}

\section{NOTES}

1. All values from IEEE Std 835-1994, IEEE Standard Power Cable Ampacity Tables.

2. Conductor Temperature: $90^{\circ} \mathrm{C}$

3. Insulation: EPR

4. Installation: Direct buried, triplexed cable

5. Load Factor: $75 \%$

6. Earth Temperature: $25^{\circ} \mathrm{C}$

7. Earth Thermal Resistivity: 90 Rho 
Table A3.5

\section{WindPACT Turbine Design Scaling Studies}

\section{Overhead Distribution Cable}

\section{Cable Type}

795 ACSR Drake / Single Circuit 795 ACSR Drake / Double Circuit

795 ACSR Drake / Single Circuit 4/0 ACSR Penguin / Single Circuit

477 ACSR Pelican / Single Circuit 2/0 ACSR Quail / Single Circuit

\section{Voltage \\ kV}

13.8

13.8

24.9

24.9

34.5

34.5

\section{Capacitance \\ (pf/ft.)}

3.59

3.86

3.59

3.14

3.37

3.01

Cable Impedance Data

(ohms/1,000 ft.)

Amps per Circuit

907

907

907

360

646

276
Zero Sequence

$0.0795+j 0.5552$

$0.0795+j 0.3714$

$0.0795+j 0.5552$

$0.1650+j 0.5850$

$0.0960+j 0.5630$ $0.2170+j 0.5920$ 
Table B1.1 Bus Description and Generator Characteristics Plan A 750 - 15 kV

Table B1.2 Distribution Cable and Overhead Line Characteristics Plan A 750 - 15 kV

Table B1.3 Transformer Characteristics Plan A 750 - 15 kV

Table B2.1 Bus Description and Generator Characteristics Plan A $750-25$ kV

Table B2.2 Distribution Cable and Overhead Line Characteristics Plan A 750 - 25 kV

Table B2.3 Transformer Characteristics Plan A 750 - 25 kV

Table B3.1 Bus Description and Generator Characteristics Plan A 2,500 - 25 kV

Table B3.2 Distribution Cable and Overhead Line Characteristics Plan A 2,500 - 25 kV

Table B3.3 Transformer Characteristics Plan A 2,500 - 25 kV

Table B4.1 Bus Description and Generator Characteristics Plan A 2,500 - 35 kV

Table B4.2 Distribution Cable and Overhead Line Characteristics Plan A 2,500 - 35 kV

Table B4.3 Transformer Characteristics Plan A 2,500 - 35 kV

Table B5.1 Bus Description and Generator Characteristics Plan A 5,000 - 25 kV

Table B5.2 Distribution Cable and Overhead Line Characteristics Plan A 5,000 - 25 kV

Table B5.3 Transformer Characteristics Plan A 5,000 - 25 kV

Table B6.1 Bus Description and Generator Characteristics Plan A 5,000 - 35 kV

Table B6.2 Distribution Cable and Overhead Line Characteristics Plan A 5,000 - 35 kV

Table B6.3 Transformer Characteristics Plan A 5,000 - 35 kV

Table B7.1 Bus Description and Generator Characteristics Plan A 10,000 - 25 kV

Table B7.2 Distribution Cable and Overhead Line Characteristics Plan A 10,000 - 25 kV

Table B7.3 Transformer Characteristics Plan A 10,000 - 25 kV

Table B8.1 Bus Description and Generator Characteristics Plan A 10,000 - 35 kV

Table B8.2 Distribution Cable and Overhead Line Characteristics Plan A 10,000 - 35 kV

Table B8.3 Transformer Characteristics Plan A 10,000 - 35 kV 
Table B1.1

Bus Description and Generator Characteristics

Plan A 750 - 15 kV

WindPACT Turbine Design Scaling Studies

\begin{tabular}{|c|c|c|}
\hline \multicolumn{3}{|c|}{ Collector System Voltage $=$} \\
\hline & & Base \\
\hline Bus No. & Name & kV \\
\hline & Mission 115 & 115.00 \\
\hline & Mission 13.8 & 13.8 \\
\hline & Riser_A & 13.8 \\
\hline & Riser_B & 13.8 \\
\hline & Riser_C & 13.8 \\
\hline & Riser_D & 13.8 \\
\hline & Riser_E & 13.8 \\
\hline 101 & Wind 01 & 13.8 \\
\hline 102 & Wind 02 & 13.8 \\
\hline 103 & Wind 03 & 13.8 \\
\hline 104 & Wind 04 & 13.8 \\
\hline 105 & Wind 05 & 13.8 \\
\hline 106 & Wind 06 & 13.8 \\
\hline 107 & Wind 07 & 13.8 \\
\hline $10 \varepsilon$ & Wind 08 & 13.8 \\
\hline $10 s$ & Wind 09 & 13.8 \\
\hline 110 & Wind_10 & 13.8 \\
\hline 111 & Wind_11 & 13.8 \\
\hline 112 & Wind_12 & 13.8 \\
\hline 113 & Wind_13 & 13.8 \\
\hline 114 & Wind_14 & 13.8 \\
\hline 115 & Wind_15 & 13.8 \\
\hline 116 & Wind_16 & 13.8 \\
\hline 117 & Wind_17 & 13.8 \\
\hline $11 \varepsilon$ & Wind 18 & 13.8 \\
\hline $11 \mathrm{c}$ & Wind 19 & 13.8 \\
\hline 120 & Wind_20 & 13.8 \\
\hline 121 & Wind 21 & 13.8 \\
\hline
\end{tabular}

13.8

Pgen

MW
Power Flow Model

$\begin{array}{ll}\text { Qmax } & \text { Qmin } \\ \text { MVAR } & \text { MVAR }\end{array}$

\begin{tabular}{|c|c|c|}
\hline \multirow{3}{*}{$\begin{array}{l}\text { Vhold } \\
1.0000\end{array}$} & \multicolumn{2}{|c|}{ Dus Iyp } \\
\hline & BType & BT \\
\hline & Reference & \\
\hline & Load & \\
\hline & Load & \\
\hline & Load & \\
\hline & Load & \\
\hline & Load & \\
\hline & Load & \\
\hline & Load & \\
\hline & Load & \\
\hline & Load & \\
\hline & Load & \\
\hline & Load & \\
\hline & Load & \\
\hline & Load & \\
\hline & Load & \\
\hline & Load & \\
\hline & Load & \\
\hline & Load & \\
\hline & Load & \\
\hline & Load & \\
\hline & Load & \\
\hline & Load & \\
\hline & Load & \\
\hline & Load & \\
\hline & Load & \\
\hline & Load & \\
\hline & Load & \\
\hline & L & \\
\hline
\end{tabular}


Table B1.1

Bus Description and Generator Characteristics

Plan A 750 - 15 kV

WindPACT Turbine Design Scaling Studies

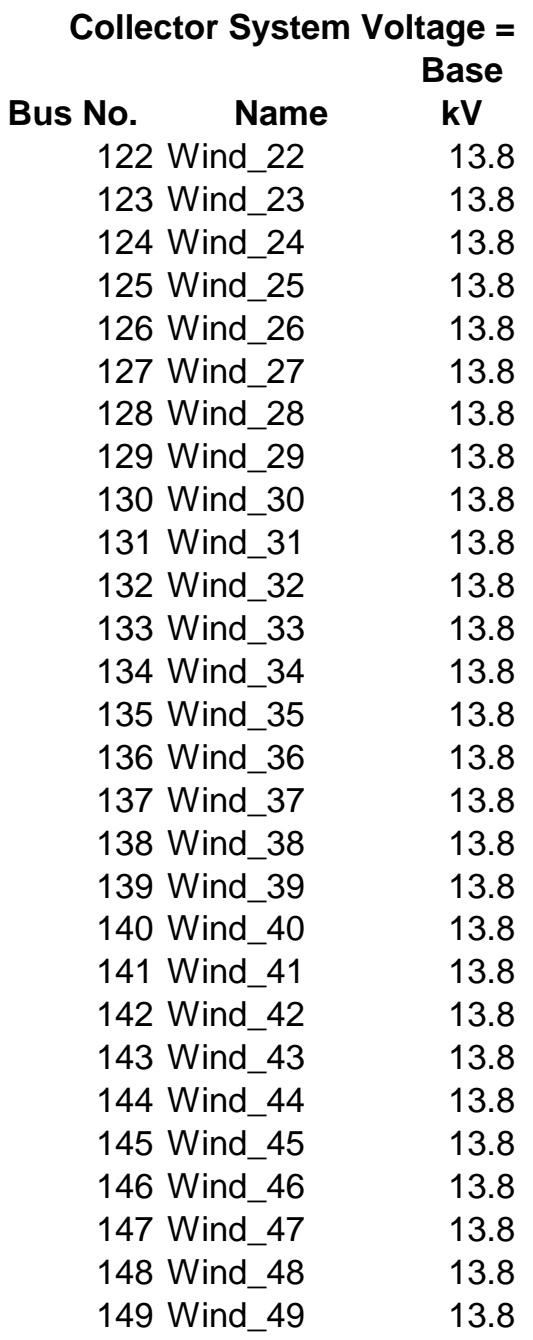

\begin{tabular}{|c|c|c|c|c|c|c|}
\hline \multirow{31}{*}{$\begin{array}{c}13.8 \\
\text { Pgen } \\
\text { MW }\end{array}$} & \multicolumn{6}{|c|}{ Power Flow Model } \\
\hline & Qmax & Qmin & & Bus & & \\
\hline & MVAR & MVAR & Vhold & BType & BT & \\
\hline & & & & Load & & 0 \\
\hline & & & & Load & & 0 \\
\hline & & & & Load & & 0 \\
\hline & & & & Load & & 0 \\
\hline & & & & Load & & 0 \\
\hline & & & & Load & & 0 \\
\hline & & & & Load & & 0 \\
\hline & & & & Load & & 0 \\
\hline & & & & Load & & 0 \\
\hline & & & & Load & & 0 \\
\hline & & & & Load & & 0 \\
\hline & & & & Load & & 0 \\
\hline & & & & Load & & 0 \\
\hline & & & & Load & & 0 \\
\hline & & & & Load & & 0 \\
\hline & & & & Load & & 0 \\
\hline & & & & Load & & 0 \\
\hline & & & & Load & & 0 \\
\hline & & & & Load & & 0 \\
\hline & & & & Load & & 0 \\
\hline & & & & Load & & 0 \\
\hline & & & & Load & & 0 \\
\hline & & & & Load & & 0 \\
\hline & & & & Load & & 0 \\
\hline & & & & Load & & 0 \\
\hline & & & & Load & & 0 \\
\hline & & & & Load & & 0 \\
\hline & & & & Load & & 0 \\
\hline
\end{tabular}


Table B1.1

Bus Description and Generator Characteristics

Plan A 750 - 15 kV

WindPACT Turbine Design Scaling Studies

$\begin{array}{cc}\text { Collector System } & \begin{array}{r}\text { Voltage }= \\ \text { Base }\end{array} \\ \text { Bus No. Name } & \text { kV } \\ \text { 150 Wind_50 } & 13.8 \\ \text { 151 Wind_51 } & 13.8 \\ \text { 152 Wind_52 } & 13.8 \\ \text { 153 Wind_53 } & 13.8 \\ \text { 154 Wind_54 } & 13.8 \\ \text { 155 Wind_55 } & 13.8 \\ \text { 156 Wind_56 } & 13.8 \\ \text { 157 Wind_57 } & 13.8 \\ \text { 158 Wind_58 } & 13.8 \\ \text { 159 Wind_59 } & 13.8 \\ \text { 160 Wind_60 } & 13.8 \\ \text { 161 Wind_61 } & 13.8 \\ \text { 162 Wind_62 } & 13.8 \\ \text { 163 Wind_63 } & 13.8 \\ \text { 164 Wind_64 } & 13.8 \\ \text { 165 Wind_65 } & 13.8 \\ \text { 166 Wind_66 } & 13.8\end{array}$

\begin{tabular}{|c|c|c|c|c|c|c|}
\hline \multirow{20}{*}{$\begin{array}{c}13.8 \\
\text { Pgen } \\
\text { MW }\end{array}$} & \multicolumn{6}{|c|}{ Power Flow Model } \\
\hline & \multirow{19}{*}{$\begin{array}{l}\text { Qmax } \\
\text { MVAR }\end{array}$} & \multirow{19}{*}{$\begin{array}{c}\text { Qmin } \\
\text { MVAR }\end{array}$} & \multirow{3}{*}{ Vhold } & \multicolumn{3}{|c|}{ Bus Type } \\
\hline & & & & ВТуре & BT & \\
\hline & & & & Load & & 0 \\
\hline & & & & Load & & 0 \\
\hline & & & & Load & & 0 \\
\hline & & & & Load & & 0 \\
\hline & & & & Load & & 0 \\
\hline & & & & Load & & 0 \\
\hline & & & & Load & & 0 \\
\hline & & & & Load & & 0 \\
\hline & & & & Load & & 0 \\
\hline & & & & Load & & 0 \\
\hline & & & & Load & & 0 \\
\hline & & & & Load & & 0 \\
\hline & & & & Load & & 0 \\
\hline & & & & Load & & 0 \\
\hline & & & & Load & & 0 \\
\hline & & & & Load & & 0 \\
\hline & & & & Load & & 0 \\
\hline
\end{tabular}


Table B1.1

Bus Description and Generator Characteristics

Plan A 750 - 15 kV

WindPACT Turbine Design Scaling Studies

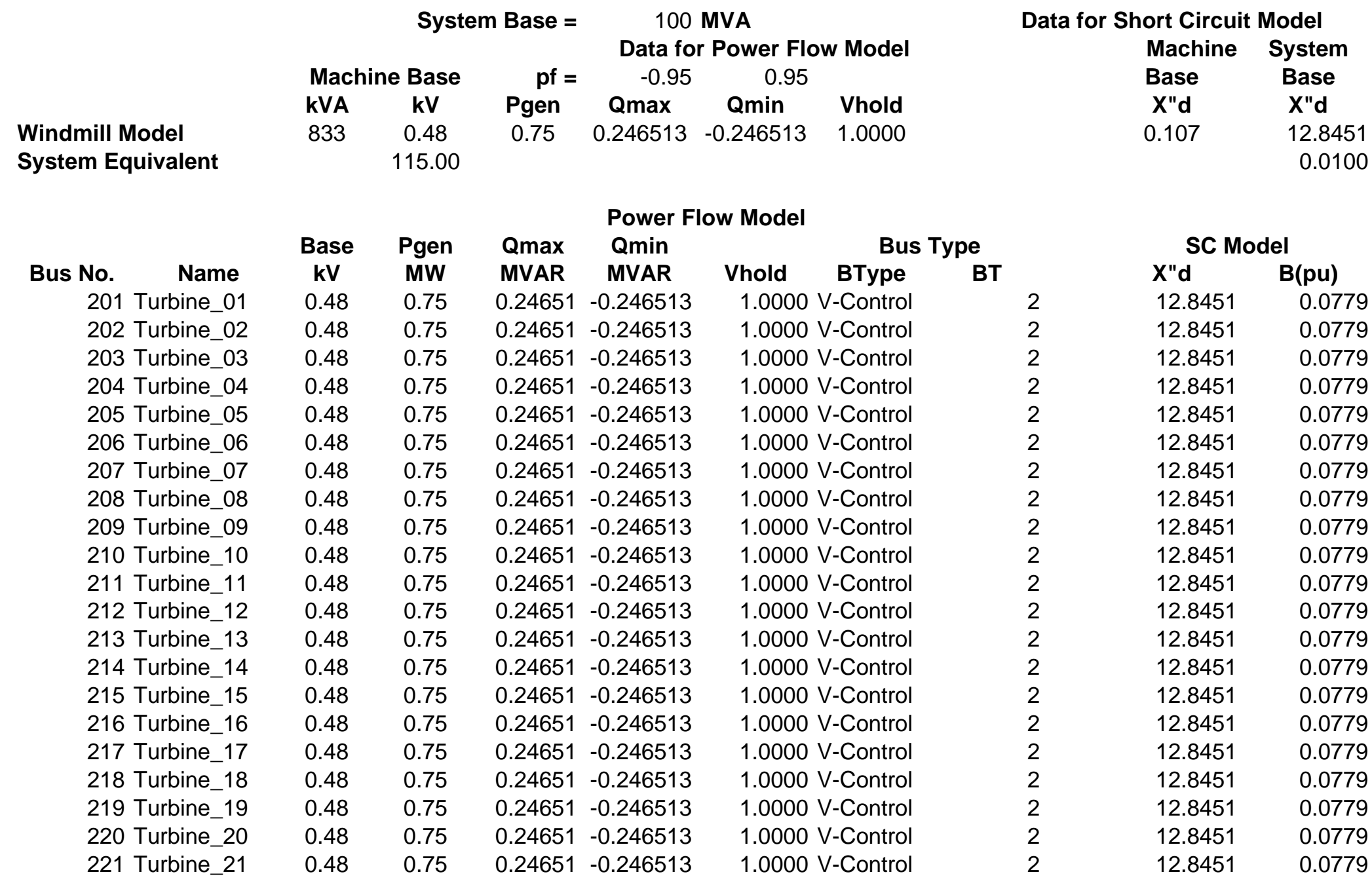


Table B1.1

Bus Description and Generator Characteristics Plan A 750 - 15 kV

\section{WindPACT Turbine Design Scaling Studies}

\begin{tabular}{|c|c|c|c|c|c|c|c|c|c|}
\hline \multirow[b]{2}{*}{ Name } & \multirow{2}{*}{$\begin{array}{c}\text { Base } \\
\mathrm{kV}\end{array}$} & \multirow{2}{*}{$\begin{array}{c}\text { Pgen } \\
\text { MW }\end{array}$} & \multirow{2}{*}{$\begin{array}{l}\text { Qmax } \\
\text { MVAR }\end{array}$} & $\begin{array}{l}\text { Power } \\
\text { Qmin }\end{array}$ & 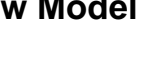 & \multicolumn{2}{|c|}{ Bus Type } & \multicolumn{2}{|c|}{ SC Model } \\
\hline & & & & MVAR & Vhold & BType & BT & X"d & $B(p u)$ \\
\hline 222 Turbine_22 & 0.48 & 0.75 & 0.24651 & -0.246513 & 1.0000 & V-Control & 2 & 12.8451 & 0.0779 \\
\hline 223 Turbine_23 & 0.48 & 0.75 & 0.24651 & -0.246513 & 1.0000 & V-Control & 2 & 12.8451 & 0.0779 \\
\hline 224 Turbine_24 & 0.48 & 0.75 & 0.24651 & -0.246513 & 1.0000 & V-Control & 2 & 12.8451 & 0.0779 \\
\hline 225 Turbine_25 & 0.48 & 0.75 & 0.24651 & -0.246513 & 1.0000 & V-Control & 2 & 12.8451 & 0.0779 \\
\hline 226 Turbine_26 & 0.48 & 0.75 & 0.24651 & -0.246513 & 1.0000 & V-Control & 2 & 12.8451 & 0.0779 \\
\hline 227 Turbine_27 & 0.48 & 0.75 & 0.24651 & -0.246513 & 1.0000 & V-Control & 2 & 12.8451 & 0.0779 \\
\hline 228 Turbine_28 & 0.48 & 0.75 & 0.24651 & -0.246513 & 1.0000 & V-Control & 2 & 12.8451 & 0.0779 \\
\hline 229 Turbine_29 & 0.48 & 0.75 & 0.24651 & -0.246513 & 1.0000 & V-Control & 2 & 12.8451 & 0.0779 \\
\hline 230 Turbine_30 & 0.48 & 0.75 & 0.24651 & -0.246513 & 1.0000 & V-Control & 2 & 12.8451 & 0.0779 \\
\hline 231 Turbine_31 & 0.48 & 0.75 & 0.24651 & -0.246513 & 1.0000 & V-Control & 2 & 12.8451 & 0.0779 \\
\hline 232 Turbine_32 & 0.48 & 0.75 & 0.24651 & -0.246513 & 1.0000 & V-Control & 2 & 12.8451 & 0.0779 \\
\hline 233 Turbine_33 & 0.48 & 0.75 & 0.24651 & -0.246513 & 1.0000 & V-Control & 2 & 12.8451 & 0.0779 \\
\hline 234 Turbine_34 & 0.48 & 0.75 & 0.24651 & -0.246513 & 1.0000 & V-Control & 2 & 12.8451 & 0.0779 \\
\hline 235 Turbine_35 & 0.48 & 0.75 & 0.24651 & -0.246513 & 1.0000 & V-Control & 2 & 12.8451 & 0.0779 \\
\hline 236 Turbine_36 & 0.48 & 0.75 & 0.24651 & -0.246513 & 1.0000 & V-Control & 2 & 12.8451 & 0.0779 \\
\hline 237 Turbine_37 & 0.48 & 0.75 & 0.24651 & -0.246513 & 1.0000 & V-Control & 2 & 12.8451 & 0.0779 \\
\hline 238 Turbine_38 & 0.48 & 0.75 & 0.24651 & -0.246513 & 1.0000 & V-Control & 2 & 12.8451 & 0.0779 \\
\hline 239 Turbine_39 & 0.48 & 0.75 & 0.24651 & -0.246513 & 1.0000 & V-Control & 2 & 12.8451 & 0.0779 \\
\hline 240 Turbine_40 & 0.48 & 0.75 & 0.24651 & -0.246513 & 1.0000 & V-Control & 2 & 12.8451 & 0.0779 \\
\hline 241 Turbine_41 & 0.48 & 0.75 & 0.24651 & -0.246513 & 1.0000 & V-Control & 2 & 12.8451 & 0.0779 \\
\hline 242 Turbine_42 & 0.48 & 0.75 & 0.24651 & -0.246513 & 1.0000 & V-Control & 2 & 12.8451 & 0.0779 \\
\hline 243 Turbine_43 & 0.48 & 0.75 & 0.24651 & -0.246513 & 1.0000 & V-Control & 2 & 12.8451 & 0.0779 \\
\hline 244 Turbine_44 & 0.48 & 0.75 & 0.24651 & -0.246513 & 1.0000 & V-Control & 2 & 12.8451 & 0.0779 \\
\hline 245 Turbine_45 & 0.48 & 0.75 & 0.24651 & -0.246513 & 1.0000 & V-Control & 2 & 12.8451 & 0.0779 \\
\hline 246 Turbine_46 & 0.48 & 0.75 & 0.24651 & -0.246513 & 1.0000 & V-Control & 2 & 12.8451 & 0.0779 \\
\hline 247 Turbine_47 & 0.48 & 0.75 & 0.24651 & -0.246513 & 1.0000 & V-Control & 2 & 12.8451 & 0.0779 \\
\hline 248 Turbine_48 & 0.48 & 0.75 & 0.24651 & -0.246513 & 1.0000 & V-Control & 2 & 12.8451 & 0.0779 \\
\hline 249 Turbine_49 & 0.48 & 0.75 & 0.24651 & -0.246513 & 1.0000 & V-Control & 2 & 12.8451 & 0.0779 \\
\hline
\end{tabular}


Table B1.1

Bus Description and Generator Characteristics Plan A 750 - 15 kV

\section{WindPACT Turbine Design Scaling Studies}

\begin{tabular}{|c|c|c|c|c|c|c|c|c|c|}
\hline & \multicolumn{7}{|c|}{$\begin{array}{l}\text { Power Flow Model } \\
\text { Qmin }\end{array}$} & \multicolumn{2}{|c|}{ SC Model } \\
\hline Bus No. & kV & MW & MVAR & MVAR & Vhold & BType & BT & X"d & $B(p u)$ \\
\hline 250 Turbine_50 & 0.48 & 0.75 & 0.24651 & -0.246513 & 1.0000 & V-Control & 2 & 12.8451 & 0.0779 \\
\hline 251 Turbine_51 & 0.48 & 0.75 & 0.24651 & -0.246513 & 1.0000 & V-Control & 2 & 12.8451 & 0.0779 \\
\hline 252 Turbine_52 & 0.48 & 0.75 & 0.24651 & -0.246513 & 1.0000 & V-Control & 2 & 12.8451 & 0.0779 \\
\hline 253 Turbine_53 & 0.48 & 0.75 & 0.24651 & -0.246513 & 1.0000 & V-Control & 2 & 12.8451 & 0.0779 \\
\hline 254 Turbine_54 & 0.48 & 0.75 & 0.24651 & -0.246513 & 1.0000 & V-Control & 2 & 12.8451 & 0.0779 \\
\hline 255 Turbine_55 & 0.48 & 0.75 & 0.24651 & -0.246513 & 1.0000 & V-Control & 2 & 12.8451 & 0.0779 \\
\hline 256 Turbine_56 & 0.48 & 0.75 & 0.24651 & -0.246513 & 1.0000 & V-Control & 2 & 12.8451 & 0.0779 \\
\hline 257 Turbine_57 & 0.48 & 0.75 & 0.24651 & -0.246513 & 1.0000 & V-Control & 2 & 12.8451 & 0.0779 \\
\hline 258 Turbine_58 & 0.48 & 0.75 & 0.24651 & -0.246513 & 1.0000 & V-Control & 2 & 12.8451 & 0.0779 \\
\hline 259 Turbine_59 & 0.48 & 0.75 & 0.24651 & -0.246513 & 1.0000 & V-Control & 2 & 12.8451 & 0.0779 \\
\hline 260 Turbine_60 & 0.48 & 0.75 & 0.24651 & -0.246513 & 1.0000 & V-Control & 2 & 12.8451 & 0.0779 \\
\hline 261 Turbine_61 & 0.48 & 0.75 & 0.24651 & -0.246513 & 1.0000 & V-Control & 2 & 12.8451 & 0.0779 \\
\hline 262 Turbine_62 & 0.48 & 0.75 & 0.24651 & -0.246513 & 1.0000 & V-Control & 2 & 12.8451 & 0.0779 \\
\hline 263 Turbine_63 & 0.48 & 0.75 & 0.24651 & -0.246513 & 1.0000 & V-Control & 2 & 12.8451 & 0.0779 \\
\hline 264 Turbine_64 & 0.48 & 0.75 & 0.24651 & -0.246513 & 1.0000 & V-Control & 2 & 12.8451 & 0.0779 \\
\hline 265 Turbine_65 & 0.48 & 0.75 & 0.24651 & -0.246513 & 1.0000 & V-Control & 2 & 12.8451 & 0.0779 \\
\hline 266 Turbine_66 & 0.48 & 0.75 & 0.24651 & -0.246513 & 1.0000 & V-Control & 2 & 12.8451 & 0.0779 \\
\hline
\end{tabular}


Table B1.2

Distribution Cable and Overhead Line Characteristics

Plan A 750 - 15 kV

WindPACT Turbine Design Scaling Studies

\begin{tabular}{|c|c|c|c|c|c|c|c|c|c|c|c|c|c|c|}
\hline \multirow{2}{*}{\multicolumn{2}{|c|}{$\begin{array}{c}\text { Construction } \\
\text { Type }\end{array}$}} & \multirow{2}{*}{$\begin{array}{c}\text { Conductor Size } \\
\text { AWG or kcmil }\end{array}$} & \multirow{2}{*}{$\begin{array}{c}\text { Rated } \\
\text { kV }\end{array}$} & ohms $/ 1$ & $00 \mathrm{ft}$. & \multicolumn{2}{|c|}{$\begin{array}{r}\text { Base MVA= } \\
\text { Volt Base= } \\
\text { Z Base= }\end{array}$} & \multicolumn{2}{|c|}{$\begin{array}{c}100 \\
13.80 \\
1.904 \\
\text { PU/1000 ft. }\end{array}$} & \multirow{2}{*}{$\begin{array}{l}\text { Rating } \\
\text { Amps }\end{array}$} & \multicolumn{2}{|c|}{ ohms/1000 ft. } & \multicolumn{2}{|c|}{ PU/1000 ft. } \\
\hline & & & & $\mathbf{R}$ & $\mathbf{X}$ & $\mathrm{pf} / \mathrm{ft}$ & $\mathbf{R}$ & \multicolumn{2}{|l|}{$\mathbf{X}$} & & Ro & $\mathbf{X O}$ & Ro & $\mathrm{XO}$ \\
\hline 1 & UG-1/0-15 & $1 / 0 \mathrm{Al}$ & 13.80 & 0.2120 & 0.0480 & 66 & 0.1113 & 0.0252 & 0.000047 & 216 & 0.543 & 0.237 & 0.285 & 0.124 \\
\hline 2 & UG-4/0-15 & 4/0 Al & 13.80 & 0.1070 & 0.0430 & 84 & 0.0562 & 0.0226 & 0.000060 & 318 & 0.335 & 0.105 & 0.176 & 0.055 \\
\hline 3 & UG-500-15 & $500 \mathrm{Al}$ & 13.80 & 0.0480 & 0.0370 & 115 & 0.0252 & 0.0194 & 0.000083 & 502 & 0.159 & 0.037 & 0.083 & 0.019 \\
\hline 4 & UG-750-15 & $750 \mathrm{Al}$ & 13.80 & 0.0340 & 0.0350 & 136 & 0.0179 & 0.0184 & 0.000098 & 604 & 0.106 & 0.025 & 0.056 & 0.013 \\
\hline 5 & UG-1000-15 & $1000 \mathrm{Al}$ & 13.80 & 0.0280 & 0.0330 & 152 & 0.0147 & 0.0173 & 0.000109 & 716 & 0.081 & 0.021 & 0.043 & 0.011 \\
\hline 6 & OH-SC-795 & 795 ACSR & & 0.0242 & 0.1135 & 3.86 & 0.0127 & 0.0596 & 0.000003 & 910 & 0.126 & 0.529 & 0.066 & 0.278 \\
\hline 7 & OH-DC2-795 & 796 ACSR & & 0.0242 & 0.1056 & 3.86 & 0.0127 & 0.0555 & 0.000003 & 910 & 0.126 & 0.492 & 0.066 & 0.258 \\
\hline
\end{tabular}

Map Code $=2$

\begin{tabular}{|c|c|c|c|c|c|c|c|c|c|c|c|c|c|c|}
\hline \multirow{2}{*}{$\begin{array}{l}\text { Sec } \\
\text { Id }\end{array}$} & \multirow{2}{*}{$\begin{array}{l}\text { Dist. } \\
\text { feet }\end{array}$} & \multirow{2}{*}{\multicolumn{2}{|c|}{$\begin{array}{c}\text { Construction } \\
\text { Type }\end{array}$}} & \multirow[b]{2}{*}{ Section Name } & \multirow{2}{*}{$\begin{array}{c}\text { Voltage } \\
\text { kV }\end{array}$} & \multirow{2}{*}{$\begin{array}{c}\text { From } \\
\text { No. }\end{array}$} & \multirow{2}{*}{$\begin{array}{r}\text { To } \\
\text { No. }\end{array}$} & \multirow{2}{*}{$\begin{array}{l}\text { Ckt. } \\
\text { No. }\end{array}$} & \multicolumn{3}{|c|}{ Section Impedance } & \multirow{2}{*}{$\begin{array}{c}\text { Rating } \\
\text { MVA }\end{array}$} & \multicolumn{2}{|c|}{ Per Unit } \\
\hline & & & & & & & & & Rpu & Xpu & $\mathrm{Bpu}$ & & Ro & XO \\
\hline $1-2$ & 377 & 1 & UG-1/0-15 & Wind_01 - Wind_02 & 13.8 & 101 & 102 & 1 & 0.0420 & 0.0095 & 0.000018 & 5 & 0.107 & 0.047 \\
\hline $2-3$ & 377 & 1 & UG-1/0-15 & Wind_02 - Wind_03 & 13.8 & 102 & 103 & 1 & 0.0420 & 0.0095 & 0.000018 & 5 & 0.107 & 0.047 \\
\hline $3-4$ & 377 & 1 & UG-1/0-15 & Wind_03 - Wind_04 & 13.8 & 103 & 104 & 1 & 0.0420 & 0.0095 & 0.000018 & 5 & 0.107 & 0.047 \\
\hline $4-5$ & 377 & 1 & UG-1/0-15 & Wind_04 - Wind_05 & 13.8 & 104 & 105 & 1 & 0.0420 & 0.0095 & 0.000018 & 5 & 0.107 & 0.047 \\
\hline $5-6$ & 377 & 1 & UG-1/0-15 & Wind_05 - Wind_06 & 13.8 & 105 & 106 & 1 & 0.0420 & 0.0095 & 0.000018 & 5 & 0.107 & 0.047 \\
\hline $6-7$ & 377 & 2 & UG-4/0-15 & Wind_06 - Wind_07 & 13.8 & 106 & 107 & 1 & 0.0212 & 0.0085 & 0.000023 & 8 & 0.066 & 0.021 \\
\hline $7-8$ & 377 & 2 & UG-4/0-15 & Wind_07 - Wind_08 & 13.8 & 107 & 108 & 1 & 0.0212 & 0.0085 & 0.000023 & 8 & 0.066 & 0.021 \\
\hline $8-9$ & 377 & 2 & UG-4/0-15 & Wind_08 - Wind_09 & 13.8 & 108 & 109 & 1 & 0.0212 & 0.0085 & 0.000023 & 8 & 0.066 & 0.021 \\
\hline $9-10$ & 377 & 2 & UG-4/0-15 & Wind_09 - Wind_10 & 13.8 & 109 & 110 & 1 & 0.0212 & 0.0085 & 0.000023 & 8 & 0.066 & 0.021 \\
\hline $10-11$ & 377 & 3 & UG-500-15 & Wind_10 - Wind_11 & 13.8 & 110 & 111 & 1 & 0.0095 & 0.0073 & 0.000031 & 12 & 0.031 & 0.007 \\
\hline $11-\mathrm{A}$ & 377 & 3 & UG-500-15 & Wind_11 - Riser_A & 13.8 & 111 & 10 & 1 & 0.0095 & 0.0073 & 0.000031 & 12 & 0.031 & 0.007 \\
\hline $12-A$ & 377 & 3 & UG-500-15 & Wind_12 - Riser_A & 13.8 & 112 & 10 & 1 & 0.0095 & 0.0073 & 0.000031 & 12 & 0.031 & 0.007 \\
\hline $12-13$ & 377 & 3 & UG-500-15 & Wind_12 - Wind_13 & 13.8 & 112 & 113 & 1 & 0.0095 & 0.0073 & 0.000031 & 12 & 0.031 & 0.007 \\
\hline $13-14$ & 377 & 2 & UG-4/0-15 & Wind_13 - Wind_14 & 13.8 & 113 & 114 & 1 & 0.0212 & 0.0085 & 0.000023 & 8 & 0.066 & 0.021 \\
\hline $14-15$ & 377 & 2 & UG-4/0-15 & Wind_14 - Wind_15 & 13.8 & 114 & 115 & 1 & 0.0212 & 0.0085 & 0.000023 & 8 & 0.066 & 0.021 \\
\hline $15-16$ & 377 & 2 & UG-4/0-15 & Wind_15 - Wind_16 & 13.8 & 115 & 116 & 1 & 0.0212 & 0.0085 & 0.000023 & 8 & 0.066 & 0.021 \\
\hline $16-17$ & 377 & 2 & UG-4/0-15 & Wind_16 - Wind_17 & 13.8 & 116 & 117 & 1 & 0.0212 & 0.0085 & 0.000023 & 8 & 0.066 & 0.021 \\
\hline $17-18$ & 377 & 1 & UG-1/0-15 & Wind_17 - Wind_18 & 13.8 & 117 & 118 & 1 & 0.0420 & 0.0095 & 0.000018 & 5 & 0.107 & 0.047 \\
\hline $18-19$ & 377 & 1 & UG-1/0-15 & Wind_18 - Wind_19 & 13.8 & 118 & 119 & 1 & 0.0420 & 0.0095 & 0.000018 & 5 & 0.107 & 0.047 \\
\hline $19-20$ & 377 & 1 & UG-1/0-15 & Wind_19-Wind_20 & 13.8 & 119 & 120 & 1 & 0.0420 & 0.0095 & 0.000018 & 5 & 0.107 & 0.047 \\
\hline
\end{tabular}


Table B1.2

Distribution Cable and Overhead Line Characteristics

Plan A 750 - 15 kV

WindPACT Turbine Design Scaling Studies

\begin{tabular}{|c|c|c|c|c|c|c|c|c|c|c|c|c|c|c|}
\hline \multirow{2}{*}{\multicolumn{2}{|c|}{$\begin{array}{c}\text { Construction } \\
\text { Type }\end{array}$}} & \multirow{2}{*}{$\begin{array}{c}\text { Conductor Size } \\
\text { AWG or kcmil }\end{array}$} & \multirow{2}{*}{$\begin{array}{c}\text { Rated } \\
\text { kV }\end{array}$} & ohms $/ 1$ & $00 \mathrm{ft}$. & \multicolumn{2}{|c|}{$\begin{array}{r}\text { Base MVA= } \\
\text { Volt Base= } \\
\text { Z Base= }\end{array}$} & \multicolumn{2}{|c|}{$\begin{array}{c}100 \\
13.80 \\
1.904 \\
\text { PU/1000 ft. }\end{array}$} & \multirow{2}{*}{$\begin{array}{l}\text { Rating } \\
\text { Amps }\end{array}$} & \multicolumn{2}{|c|}{ ohms/1000 ft. } & \multicolumn{2}{|c|}{ PU/1000 ft. } \\
\hline & & & & $\mathbf{R}$ & $\mathbf{X}$ & $\mathrm{pf} / \mathrm{ft}$ & $\mathbf{R}$ & \multicolumn{2}{|l|}{$\mathbf{X}$} & & Ro & $\mathbf{X O}$ & Ro & $\mathrm{XO}$ \\
\hline 1 & UG-1/0-15 & $1 / 0 \mathrm{Al}$ & 13.80 & 0.2120 & 0.0480 & 66 & 0.1113 & 0.0252 & 0.000047 & 216 & 0.543 & 0.237 & 0.285 & 0.124 \\
\hline 2 & UG-4/0-15 & 4/0 Al & 13.80 & 0.1070 & 0.0430 & 84 & 0.0562 & 0.0226 & 0.000060 & 318 & 0.335 & 0.105 & 0.176 & 0.055 \\
\hline 3 & UG-500-15 & $500 \mathrm{Al}$ & 13.80 & 0.0480 & 0.0370 & 115 & 0.0252 & 0.0194 & 0.000083 & 502 & 0.159 & 0.037 & 0.083 & 0.019 \\
\hline 4 & UG-750-15 & $750 \mathrm{Al}$ & 13.80 & 0.0340 & 0.0350 & 136 & 0.0179 & 0.0184 & 0.000098 & 604 & 0.106 & 0.025 & 0.056 & 0.013 \\
\hline 5 & UG-1000-15 & $1000 \mathrm{Al}$ & 13.80 & 0.0280 & 0.0330 & 152 & 0.0147 & 0.0173 & 0.000109 & 716 & 0.081 & 0.021 & 0.043 & 0.011 \\
\hline 6 & OH-SC-795 & 795 ACSR & & 0.0242 & 0.1135 & 3.86 & 0.0127 & 0.0596 & 0.000003 & 910 & 0.126 & 0.529 & 0.066 & 0.278 \\
\hline 7 & OH-DC2-795 & 796 ACSR & & 0.0242 & 0.1056 & 3.86 & 0.0127 & 0.0555 & 0.000003 & 910 & 0.126 & 0.492 & 0.066 & 0.258 \\
\hline
\end{tabular}

Map Code $=2$

\begin{tabular}{|c|c|c|c|c|c|c|c|c|c|c|c|c|c|c|}
\hline \multirow{2}{*}{$\begin{array}{l}\text { Sec } \\
\text { Id }\end{array}$} & \multirow{2}{*}{$\begin{array}{l}\text { Dist. } \\
\text { feet }\end{array}$} & \multirow{2}{*}{\multicolumn{2}{|c|}{$\begin{array}{c}\text { Construction } \\
\text { Type }\end{array}$}} & \multirow[b]{2}{*}{ Section Name } & \multirow{2}{*}{$\begin{array}{c}\text { Voltage } \\
\text { kV }\end{array}$} & \multirow{2}{*}{$\begin{array}{l}\text { From } \\
\text { No. }\end{array}$} & \multirow{2}{*}{$\begin{array}{l}\text { To } \\
\text { No. }\end{array}$} & \multirow{2}{*}{$\begin{array}{l}\text { Ckt. } \\
\text { No. }\end{array}$} & \multicolumn{3}{|c|}{ Section Impedance } & \multirow{2}{*}{$\begin{array}{l}\text { Rating } \\
\text { MVA }\end{array}$} & \multicolumn{2}{|c|}{ Per Unit } \\
\hline & & & & & & & & & Rpu & Xpu & Bpu & & Ro & XO \\
\hline $20-21$ & 377 & 1 & UG-1/0-15 & Wind_20 - Wind_21 & 13.8 & 120 & 121 & 1 & 0.0420 & 0.0095 & 0.000018 & 5 & 0.107 & 0.047 \\
\hline $21-22$ & 377 & 1 & UG-1/0-15 & Wind_21 - Wind_22 & 13.8 & 121 & 122 & 1 & 0.0420 & 0.0095 & 0.000018 & 5 & 0.107 & 0.047 \\
\hline $22-23$ & 377 & 1 & UG-1/0-15 & Wind_22 - Wind_23 & 13.8 & 122 & 123 & 1 & 0.0420 & 0.0095 & 0.000018 & 5 & 0.107 & 0.047 \\
\hline $24-25$ & 377 & 1 & UG-1/0-15 & Wind_24 - Wind_25 & 13.8 & 124 & 125 & 1 & 0.0420 & 0.0095 & 0.000018 & 5 & 0.107 & 0.047 \\
\hline $25-26$ & 377 & 1 & UG-1/0-15 & Wind_25 - Wind_26 & 13.8 & 125 & 126 & 1 & 0.0420 & 0.0095 & 0.000018 & 5 & 0.107 & 0.047 \\
\hline $26-27$ & 377 & 1 & UG-1/0-15 & Wind_26 - Wind_27 & 13.8 & 126 & 127 & 1 & 0.0420 & 0.0095 & 0.000018 & 5 & 0.107 & 0.047 \\
\hline $27-28$ & 377 & 1 & UG-1/0-15 & Wind_27 - Wind_28 & 13.8 & 127 & 128 & 1 & 0.0420 & 0.0095 & 0.000018 & 5 & 0.107 & 0.047 \\
\hline $28-29$ & 377 & 1 & UG-1/0-15 & Wind_28 - Wind_29 & 13.8 & 128 & 129 & 1 & 0.0420 & 0.0095 & 0.000018 & 5 & 0.107 & 0.047 \\
\hline $29-B$ & 377 & 2 & UG-4/0-15 & Wind_29 - Riser_B & 13.8 & 129 & 15 & 1 & 0.0212 & 0.0085 & 0.000023 & 8 & 0.066 & 0.021 \\
\hline $30-C$ & 377 & 3 & UG-500-15 & Wind_30 - Riser_C & 13.8 & 130 & 20 & 1 & 0.0095 & 0.0073 & 0.000031 & 12 & 0.031 & 0.007 \\
\hline $30-31$ & 377 & 3 & UG-500-15 & Wind_30 - Wind_31 & 13.8 & 130 & 131 & 1 & 0.0095 & 0.0073 & 0.000031 & 12 & 0.031 & 0.007 \\
\hline $31-32$ & 377 & 3 & UG-500-15 & Wind_31 - Wind_32 & 13.8 & 131 & 132 & 1 & 0.0095 & 0.0073 & 0.000031 & 12 & 0.031 & 0.007 \\
\hline $32-33$ & 377 & 3 & UG-500-15 & Wind_32 - Wind_33 & 13.8 & 132 & 133 & 1 & 0.0095 & 0.0073 & 0.000031 & 12 & 0.031 & 0.007 \\
\hline $33-34$ & 377 & 3 & UG-500-15 & Wind_33 - Wind_34 & 13.8 & 133 & 134 & 1 & 0.0095 & 0.0073 & 0.000031 & 12 & 0.031 & 0.007 \\
\hline $34-35$ & 377 & 3 & UG-500-15 & Wind_34 - Wind_35 & 13.8 & 134 & 135 & 1 & 0.0095 & 0.0073 & 0.000031 & 12 & 0.031 & 0.007 \\
\hline $35-36$ & 377 & 3 & UG-500-15 & Wind_35 - Wind_36 & 13.8 & 135 & 136 & 1 & 0.0095 & 0.0073 & 0.000031 & 12 & 0.031 & 0.007 \\
\hline $36-37$ & 377 & 2 & UG-4/0-15 & Wind_36 - Wind_37 & 13.8 & 136 & 137 & 1 & 0.0212 & 0.0085 & 0.000023 & 8 & 0.066 & 0.021 \\
\hline $37-38$ & 377 & 2 & UG-4/0-15 & Wind_37 - Wind_38 & 13.8 & 137 & 138 & 1 & 0.0212 & 0.0085 & 0.000023 & 8 & 0.066 & 0.021 \\
\hline $38-39$ & 377 & 2 & UG-4/0-15 & Wind_38 - Wind_39 & 13.8 & 138 & 139 & 1 & 0.0212 & 0.0085 & 0.000023 & 8 & 0.066 & 0.021 \\
\hline $39-40$ & 377 & 1 & UG-1/0-15 & Wind_39-Wind_40 & 13.8 & 139 & 140 & 1 & 0.0420 & 0.0095 & 0.000018 & 5 & 0.107 & 0.047 \\
\hline
\end{tabular}


Table B1.2

Distribution Cable and Overhead Line Characteristics

Plan A 750 - 15 kV

WindPACT Turbine Design Scaling Studies

\begin{tabular}{|c|c|c|c|c|c|c|c|c|c|c|c|c|c|c|}
\hline \multirow{2}{*}{\multicolumn{2}{|c|}{$\begin{array}{c}\text { Construction } \\
\text { Type }\end{array}$}} & \multirow{2}{*}{$\begin{array}{c}\text { Conductor Size } \\
\text { AWG or kcmil }\end{array}$} & \multirow{2}{*}{$\begin{array}{c}\text { Rated } \\
\text { kV }\end{array}$} & ohms $/ 1$ & $00 \mathrm{ft}$. & \multicolumn{2}{|c|}{$\begin{array}{r}\text { Base MVA= } \\
\text { Volt Base= } \\
\text { Z Base= }\end{array}$} & \multicolumn{2}{|c|}{$\begin{array}{c}100 \\
13.80 \\
1.904 \\
\text { PU/1000 ft. }\end{array}$} & \multirow{2}{*}{$\begin{array}{l}\text { Rating } \\
\text { Amps }\end{array}$} & \multicolumn{2}{|c|}{ ohms/1000 ft. } & \multicolumn{2}{|c|}{ PU/1000 ft. } \\
\hline & & & & $\mathbf{R}$ & $\mathbf{X}$ & $\mathrm{pf} / \mathrm{ft}$ & $\mathbf{R}$ & \multicolumn{2}{|l|}{$\mathbf{X}$} & & Ro & $\mathbf{X O}$ & Ro & $\mathrm{XO}$ \\
\hline 1 & UG-1/0-15 & $1 / 0 \mathrm{Al}$ & 13.80 & 0.2120 & 0.0480 & 66 & 0.1113 & 0.0252 & 0.000047 & 216 & 0.543 & 0.237 & 0.285 & 0.124 \\
\hline 2 & UG-4/0-15 & 4/0 Al & 13.80 & 0.1070 & 0.0430 & 84 & 0.0562 & 0.0226 & 0.000060 & 318 & 0.335 & 0.105 & 0.176 & 0.055 \\
\hline 3 & UG-500-15 & $500 \mathrm{Al}$ & 13.80 & 0.0480 & 0.0370 & 115 & 0.0252 & 0.0194 & 0.000083 & 502 & 0.159 & 0.037 & 0.083 & 0.019 \\
\hline 4 & UG-750-15 & $750 \mathrm{Al}$ & 13.80 & 0.0340 & 0.0350 & 136 & 0.0179 & 0.0184 & 0.000098 & 604 & 0.106 & 0.025 & 0.056 & 0.013 \\
\hline 5 & UG-1000-15 & $1000 \mathrm{Al}$ & 13.80 & 0.0280 & 0.0330 & 152 & 0.0147 & 0.0173 & 0.000109 & 716 & 0.081 & 0.021 & 0.043 & 0.011 \\
\hline 6 & OH-SC-795 & 795 ACSR & & 0.0242 & 0.1135 & 3.86 & 0.0127 & 0.0596 & 0.000003 & 910 & 0.126 & 0.529 & 0.066 & 0.278 \\
\hline 7 & OH-DC2-795 & 796 ACSR & & 0.0242 & 0.1056 & 3.86 & 0.0127 & 0.0555 & 0.000003 & 910 & 0.126 & 0.492 & 0.066 & 0.258 \\
\hline
\end{tabular}

Map Code $=2$

\begin{tabular}{|c|c|c|c|c|c|c|c|c|c|c|c|c|c|c|}
\hline \multirow{2}{*}{$\begin{array}{l}\text { Sec } \\
\text { Id }\end{array}$} & \multirow{2}{*}{$\begin{array}{l}\text { Dist. } \\
\text { feet }\end{array}$} & \multirow{2}{*}{\multicolumn{2}{|c|}{$\begin{array}{c}\text { Construction } \\
\text { Type }\end{array}$}} & \multirow[b]{2}{*}{ Section Name } & \multirow{2}{*}{$\begin{array}{c}\text { Voltage } \\
\text { kV }\end{array}$} & \multirow{2}{*}{$\begin{array}{l}\text { From } \\
\text { No. }\end{array}$} & \multirow{2}{*}{$\begin{array}{l}\text { To } \\
\text { No. }\end{array}$} & \multirow{2}{*}{$\begin{array}{l}\text { Ckt. } \\
\text { No. }\end{array}$} & \multicolumn{3}{|c|}{ Section Impedance } & \multirow{2}{*}{$\begin{array}{l}\text { Rating } \\
\text { MVA }\end{array}$} & \multicolumn{2}{|c|}{ Per Unit } \\
\hline & & & & & & & & & Rpu & Xpu & Bpu & & Ro & XO \\
\hline $40-41$ & 377 & 1 & UG-1/0-15 & Wind_40 - Wind_41 & 13.8 & 140 & 141 & 1 & 0.0420 & 0.0095 & 0.000018 & 5 & 0.107 & 0.047 \\
\hline $41-42$ & 377 & 1 & UG-1/0-15 & Wind_41 - Wind_42 & 13.8 & 141 & 142 & 1 & 0.0420 & 0.0095 & 0.000018 & 5 & 0.107 & 0.047 \\
\hline $42-43$ & 377 & 1 & UG-1/0-15 & Wind_42 - Wind_43 & 13.8 & 142 & 143 & 1 & 0.0420 & 0.0095 & 0.000018 & 5 & 0.107 & 0.047 \\
\hline $43-44$ & 377 & 1 & UG-1/0-15 & Wind_43 - Wind_44 & 13.8 & 143 & 144 & 1 & 0.0420 & 0.0095 & 0.000018 & 5 & 0.107 & 0.047 \\
\hline $44-45$ & 377 & 1 & UG-1/0-15 & Wind_44 - Wind_45 & 13.8 & 144 & 145 & 1 & 0.0420 & 0.0095 & 0.000018 & 5 & 0.107 & 0.047 \\
\hline $46-47$ & 377 & 1 & UG-1/0-15 & Wind_46 - Wind_47 & 13.8 & 146 & 147 & 1 & 0.0420 & 0.0095 & 0.000018 & 5 & 0.107 & 0.047 \\
\hline $47-48$ & 377 & 1 & UG-1/0-15 & Wind_47 - Wind_48 & 13.8 & 147 & 148 & 1 & 0.0420 & 0.0095 & 0.000018 & 5 & 0.107 & 0.047 \\
\hline $48-\mathrm{D}$ & 377 & 1 & UG-1/0-15 & Wind_48 - Riser_D & 13.8 & 148 & 25 & 1 & 0.0420 & 0.0095 & 0.000018 & 5 & 0.107 & 0.047 \\
\hline $49-E$ & 377 & 4 & UG-750-15 & Wind_49 - Riser_E & 13.8 & 149 & 30 & 1 & 0.0067 & 0.0069 & 0.000037 & 14 & 0.021 & 0.005 \\
\hline $49-50$ & 377 & 4 & UG-750-15 & Wind_49 - Wind_50 & 13.8 & 149 & 150 & 1 & 0.0067 & 0.0069 & 0.000037 & 14 & 0.021 & 0.005 \\
\hline $50-51$ & 377 & 4 & UG-750-15 & Wind_50 - Wind_51 & 13.8 & 150 & 151 & 1 & 0.0067 & 0.0069 & 0.000037 & 14 & 0.021 & 0.005 \\
\hline $51-52$ & 377 & 4 & UG-750-15 & Wind_51 - Wind_52 & 13.8 & 151 & 152 & 1 & 0.0067 & 0.0069 & 0.000037 & 14 & 0.021 & 0.005 \\
\hline $52-53$ & 377 & 3 & UG-500-15 & Wind_52 - Wind_53 & 13.8 & 152 & 153 & 1 & 0.0095 & 0.0073 & 0.000031 & 12 & 0.031 & 0.007 \\
\hline $53-54$ & 377 & 3 & UG-500-15 & Wind_53 - Wind_54 & 13.8 & 153 & 154 & 1 & 0.0095 & 0.0073 & 0.000031 & 12 & 0.031 & 0.007 \\
\hline $54-55$ & 377 & 3 & UG-500-15 & Wind_54 - Wind_55 & 13.8 & 154 & 155 & 1 & 0.0095 & 0.0073 & 0.000031 & 12 & 0.031 & 0.007 \\
\hline $55-56$ & 377 & 3 & UG-500-15 & Wind_55 - Wind_56 & 13.8 & 155 & 156 & 1 & 0.0095 & 0.0073 & 0.000031 & 12 & 0.031 & 0.007 \\
\hline $56-57$ & 754 & 3 & UG-500-15 & Wind_56 - Wind_57 & 13.8 & 156 & 157 & 1 & 0.0190 & 0.0146 & 0.000062 & 12 & 0.063 & 0.015 \\
\hline $57-58$ & 377 & 2 & UG-4/0-15 & Wind_57 - Wind_58 & 13.8 & 157 & 158 & 1 & 0.0212 & 0.0085 & 0.000023 & 8 & 0.066 & 0.021 \\
\hline $58-59$ & 377 & 2 & UG-4/0-15 & Wind_58 - Wind_59 & 13.8 & 158 & 159 & 1 & 0.0212 & 0.0085 & 0.000023 & 8 & 0.066 & 0.021 \\
\hline $59-60$ & 377 & 2 & UG-4/0-15 & Wind_59 - Wind_60 & 13.8 & 159 & 160 & 1 & 0.0212 & 0.0085 & 0.000023 & 8 & 0.066 & 0.021 \\
\hline
\end{tabular}


Table B1.2

Distribution Cable and Overhead Line Characteristics

Plan A 750 - 15 kV

WindPACT Turbine Design Scaling Studies

\begin{tabular}{|c|c|c|c|c|c|c|c|c|c|c|c|c|c|c|}
\hline \multirow{2}{*}{\multicolumn{2}{|c|}{$\begin{array}{c}\text { Construction } \\
\text { Type }\end{array}$}} & \multirow{2}{*}{$\begin{array}{l}\text { Conductor Size } \\
\text { AWG or kcmil }\end{array}$} & \multirow{2}{*}{$\begin{array}{c}\text { Rated } \\
\text { kV }\end{array}$} & ohms/1 & $00 \mathrm{ft}$. & \multicolumn{2}{|c|}{$\begin{array}{r}\text { Base MVA= } \\
\text { Volt Base= } \\
\text { Z Base }=\end{array}$} & \multicolumn{2}{|c|}{$\begin{array}{r}100 \\
13.80 \\
1.904\end{array}$} & \multirow{2}{*}{$\begin{array}{l}\text { Rating } \\
\text { Amps }\end{array}$} & \multicolumn{2}{|c|}{ ohms/1000 ft. } & \multicolumn{2}{|c|}{ PU/1000 ft. } \\
\hline & & & & $\bar{R}$ & $\bar{X}$ & $\mathrm{pf} / \mathrm{ft}$ & \multirow{2}{*}{$\begin{array}{c}\mathbf{R} \\
0.1113\end{array}$} & \multirow{2}{*}{$\frac{X}{0.0252}$} & \multirow{2}{*}{$\begin{array}{c}\text { B } \\
0.000047\end{array}$} & & R0 & $\mathbf{X 0}$ & R0 & $\mathbf{X O}$ \\
\hline 1 & UG-1/0-15 & $1 / 0 \mathrm{Al}$ & 13.80 & 0.2120 & 0.0480 & 66 & & & & 216 & 0.543 & 0.237 & 0.285 & 0.124 \\
\hline 2 & UG-4/0-15 & 4/0 Al & 13.80 & 0.1070 & 0.0430 & 84 & 0.0562 & 0.0226 & 0.000060 & 318 & 0.335 & 0.105 & 0.176 & 0.055 \\
\hline 3 & UG-500-15 & $500 \mathrm{Al}$ & 13.80 & 0.0480 & 0.0370 & 115 & 0.0252 & 0.0194 & 0.000083 & 502 & 0.159 & 0.037 & 0.083 & 0.019 \\
\hline 4 & UG-750-15 & $750 \mathrm{Al}$ & 13.80 & 0.0340 & 0.0350 & 136 & 0.0179 & 0.0184 & 0.000098 & 604 & 0.106 & 0.025 & 0.056 & 0.013 \\
\hline 5 & UG-1000-15 & $1000 \mathrm{Al}$ & 13.80 & 0.0280 & 0.0330 & 152 & 0.0147 & 0.0173 & 0.000109 & 716 & 0.081 & 0.021 & 0.043 & 0.011 \\
\hline 6 & OH-SC-795 & 795 ACSR & & 0.0242 & 0.1135 & 3.86 & 0.0127 & 0.0596 & 0.000003 & 910 & 0.126 & 0.529 & 0.066 & 0.278 \\
\hline 7 & OH-DC2-795 & 796 ACSR & & 0.0242 & 0.1056 & 3.86 & 0.0127 & 0.0555 & 0.000003 & 910 & 0.126 & 0.492 & 0.066 & 0.258 \\
\hline
\end{tabular}

Map Code $=2$

\begin{tabular}{|c|c|c|c|c|c|c|c|c|c|c|c|c|}
\hline \multirow{2}{*}{$\begin{array}{l}\text { Sec } \\
\text { Id }\end{array}$} & \multirow{2}{*}{$\begin{array}{l}\text { Dist. } \\
\text { feet }\end{array}$} & \multirow{2}{*}{\multicolumn{2}{|c|}{$\begin{array}{c}\text { Construction } \\
\text { Type }\end{array}$}} & \multirow[b]{2}{*}{ Section Name } & \multirow{2}{*}{$\begin{array}{c}\text { Voltage } \\
\text { kV }\end{array}$} & \multirow{2}{*}{$\begin{array}{c}\text { From } \\
\text { No. }\end{array}$} & \multirow{2}{*}{$\begin{array}{r}\text { To } \\
\text { No. }\end{array}$} & \multirow{2}{*}{$\begin{array}{l}\text { Ckt. } \\
\text { No. }\end{array}$} & \multicolumn{3}{|c|}{ Section Impedance } & \multirow{2}{*}{$\begin{array}{c}\text { Rating } \\
\text { MVA }\end{array}$} \\
\hline & & & & & & & & & Rpu & $\mathrm{Xpu}$ & Bpu & \\
\hline 60-61 & 377 & 2 & UG-4/0-15 & Wind_60 - Wind_61 & 13.8 & 160 & 161 & 1 & 0.0212 & 0.0085 & 0.000023 & 8 \\
\hline $61-62$ & 377 & 1 & UG-1/0-15 & Wind_61 - Wind_62 & 13.8 & 161 & 162 & 1 & 0.0420 & 0.0095 & 0.000018 & 5 \\
\hline $62-63$ & 377 & 1 & UG-1/0-15 & Wind_62 - Wind_63 & 13.8 & 162 & 163 & 1 & 0.0420 & 0.0095 & 0.000018 & 5 \\
\hline $63-64$ & 754 & 1 & UG-1/0-15 & Wind_63 - Wind_64 & 13.8 & 163 & 164 & 1 & 0.0839 & 0.0190 & 0.000036 & 5 \\
\hline $64-65$ & 377 & 1 & UG-1/0-15 & Wind_64 - Wind_65 & 13.8 & 164 & 165 & 1 & 0.0420 & 0.0095 & 0.000018 & 5 \\
\hline $65-66$ & 377 & 1 & UG-1/0-15 & Wind_65 - Wind_66 & 13.8 & 165 & 166 & 1 & 0.0420 & 0.0095 & 0.000018 & 5 \\
\hline
\end{tabular}

Per Unit

RO $\mathrm{XO}$

$0.066 \quad 0.021$

$0.107 \quad 0.047$

$0.107 \quad 0.047$

$0.215-0.094$

$0.107 \quad 0.047$

$0.107 \quad 0.047$ 
Table B1.2

Distribution Cable and Overhead Line Characteristics

Plan A 750 - 15 kV

WindPACT Turbine Design Scaling Studies

\begin{tabular}{|c|c|c|c|c|c|c|c|c|c|c|c|c|c|c|}
\hline \multirow{2}{*}{\multicolumn{2}{|c|}{$\begin{array}{c}\text { Construction } \\
\text { Type }\end{array}$}} & \multirow{2}{*}{$\begin{array}{l}\text { Conductor Size } \\
\text { AWG or kcmil }\end{array}$} & \multirow{2}{*}{$\begin{array}{c}\text { Rated } \\
\text { kV }\end{array}$} & ohms/1 & $00 \mathrm{ft}$. & \multicolumn{2}{|c|}{$\begin{array}{r}\text { Base MVA= } \\
\text { Volt Base= } \\
\text { Z Base }=\end{array}$} & \multicolumn{2}{|c|}{$\begin{array}{r}100 \\
13.80 \\
1.904\end{array}$} & \multirow{2}{*}{$\begin{array}{l}\text { Rating } \\
\text { Amps }\end{array}$} & \multicolumn{2}{|c|}{ ohms/1000 ft. } & \multicolumn{2}{|c|}{ PU/1000 ft. } \\
\hline & & & & $\bar{R}$ & $\bar{X}$ & $\mathrm{pf} / \mathrm{ft}$ & \multirow{2}{*}{$\begin{array}{c}\mathbf{R} \\
0.1113\end{array}$} & \multirow{2}{*}{$\frac{X}{0.0252}$} & \multirow{2}{*}{$\begin{array}{c}\text { B } \\
0.000047\end{array}$} & & R0 & $\mathbf{X 0}$ & R0 & $\mathbf{X O}$ \\
\hline 1 & UG-1/0-15 & $1 / 0 \mathrm{Al}$ & 13.80 & 0.2120 & 0.0480 & 66 & & & & 216 & 0.543 & 0.237 & 0.285 & 0.124 \\
\hline 2 & UG-4/0-15 & 4/0 Al & 13.80 & 0.1070 & 0.0430 & 84 & 0.0562 & 0.0226 & 0.000060 & 318 & 0.335 & 0.105 & 0.176 & 0.055 \\
\hline 3 & UG-500-15 & $500 \mathrm{Al}$ & 13.80 & 0.0480 & 0.0370 & 115 & 0.0252 & 0.0194 & 0.000083 & 502 & 0.159 & 0.037 & 0.083 & 0.019 \\
\hline 4 & UG-750-15 & $750 \mathrm{Al}$ & 13.80 & 0.0340 & 0.0350 & 136 & 0.0179 & 0.0184 & 0.000098 & 604 & 0.106 & 0.025 & 0.056 & 0.013 \\
\hline 5 & UG-1000-15 & $1000 \mathrm{Al}$ & 13.80 & 0.0280 & 0.0330 & 152 & 0.0147 & 0.0173 & 0.000109 & 716 & 0.081 & 0.021 & 0.043 & 0.011 \\
\hline 6 & OH-SC-795 & 795 ACSR & & 0.0242 & 0.1135 & 3.86 & 0.0127 & 0.0596 & 0.000003 & 910 & 0.126 & 0.529 & 0.066 & 0.278 \\
\hline 7 & OH-DC2-795 & 796 ACSR & & 0.0242 & 0.1056 & 3.86 & 0.0127 & 0.0555 & 0.000003 & 910 & 0.126 & 0.492 & 0.066 & 0.258 \\
\hline
\end{tabular}

Map Code $=2$

\begin{tabular}{|c|c|c|c|c|c|c|c|c|c|c|c|c|c|c|}
\hline \multirow{2}{*}{$\begin{array}{c}\mathrm{Sec} \\
\text { Id }\end{array}$} & \multirow{2}{*}{$\begin{array}{l}\text { Dist. } \\
\text { feet }\end{array}$} & \multirow{2}{*}{\multicolumn{2}{|c|}{$\begin{array}{c}\text { Construction } \\
\text { Type }\end{array}$}} & \multirow[b]{2}{*}{ Section Name } & \multirow{2}{*}{$\begin{array}{c}\text { Voltage } \\
\text { kV }\end{array}$} & \multirow{2}{*}{$\begin{array}{c}\text { From } \\
\text { No. }\end{array}$} & \multirow{2}{*}{$\begin{array}{r}\text { To } \\
\text { No. }\end{array}$} & \multirow{2}{*}{$\begin{array}{l}\text { Ckt. } \\
\text { No. }\end{array}$} & \multicolumn{3}{|c|}{ Section Impedance } & \multirow{2}{*}{$\begin{array}{c}\text { Rating } \\
\text { MVA }\end{array}$} & \multicolumn{2}{|c|}{ Per Unit } \\
\hline & & & & & & & & & Rpu & Xpu & Bpu & & Ro & XO \\
\hline$A-B$ & 2,300 & 6 & $\mathrm{OH}-\mathrm{SC}-795$ & Riser_A - Riser_B & 13.8 & 10 & 15 & 1 & 0.0292 & 0.1371 & 0.000006 & 22 & 0.152 & 0.639 \\
\hline B-D & 2,300 & 7 & $\mathrm{OH}-\mathrm{DC} 2-795$ & Riser_B - Riser_D & 13.8 & 15 & 25 & 1 & 0.0292 & 0.1275 & 0.000006 & 22 & 0.152 & 0.594 \\
\hline$D-F$ & 1,000 & 7 & OH-DC2-795 & Riser_D - Mission_13.\& & 13.8 & 25 & 2 & 1 & 0.0127 & 0.0555 & 0.000003 & 22 & 0.066 & 0.258 \\
\hline
\end{tabular}




\section{Table B1.3 \\ Transformer Characteristics \\ Plan A 750 - 15 kV \\ WindPACT Turbine Design Scaling Studies}

Transformer Characteristics

Transf. Model Data

\begin{tabular}{|c|c|c|c|c|c|c|c|}
\hline \multirow[b]{4}{*}{ Transformer } & \multirow[b]{4}{*}{ Voltage kV } & \multirow{4}{*}{$\begin{array}{l}\text { Base } \\
\text { MVA }\end{array}$} & \multirow{4}{*}{$\begin{array}{c}\text { Top } \\
\text { Rating }\end{array}$} & \multirow{2}{*}{\multicolumn{4}{|c|}{ Cuntam Parn My }} \\
\hline & & & & & stem & & 100 \\
\hline & & & & & & Imped. ( & t. Base) \\
\hline & & & & Z\% & $X / R$ & $\mathrm{R}(\mathrm{pu})$ & $\mathrm{X}(\mathrm{pu})$ \\
\hline Station Transformer & $115 / 13.8$ & 30 & 50 & $8.5 \%$ & 19 & 0.0149 & 0.2833 \\
\hline Wind Mill Transformer & $13.80 / .480$ & 1 & 1 & $5.75 \%$ & 9 & 0.6389 & 5.7500 \\
\hline
\end{tabular}

\begin{tabular}{lcccccccc} 
& & Rating & Tap & To & Ckt. & \multicolumn{2}{c}{ Imped. (Syst. Base) } & \\
\cline { 6 - 7 } Transformer Name & Voltage kV & MVA & No. & No. & Id. & R(pu) & X(pu) & Tap \\
\hline Mission_115 - Mission_13.8 & $115 / 13.8$ & 50 & 1 & 2 & 1 & 0.0149 & 0.2833 & 1.000 \\
Wind_01 - Turbine_01 & $13.80 / .480$ & 1 & 101 & 201 & 1 & 0.6389 & 5.7500 & 1.000 \\
Wind_02 - Turbine_02 & $13.80 / .480$ & 1 & 102 & 202 & 1 & 0.6389 & 5.7500 & 1.000 \\
Wind_03 - Turbine_03 & $13.80 / .480$ & 1 & 103 & 203 & 1 & 0.6389 & 5.7500 & 1.000 \\
Wind_04 - Turbine_04 & $13.80 / .480$ & 1 & 104 & 204 & 1 & 0.6389 & 5.7500 & 1.000 \\
Wind_05 - Turbine_05 & $13.80 / .480$ & 1 & 105 & 205 & 1 & 0.6389 & 5.7500 & 1.000 \\
Wind_06 - Turbine_06 & $13.80 / .480$ & 1 & 106 & 206 & 1 & 0.6389 & 5.7500 & 1.000 \\
Wind_07 - Turbine_07 & $13.80 / .480$ & 1 & 107 & 207 & 1 & 0.6389 & 5.7500 & 1.000 \\
Wind_08 - Turbine_08 & $13.80 / .480$ & 1 & 108 & 208 & 1 & 0.6389 & 5.7500 & 1.000 \\
Wind_09 - Turbine_09 & $13.80 / .480$ & 1 & 109 & 209 & 1 & 0.6389 & 5.7500 & 1.000 \\
Wind_10 - Turbine_10 & $13.80 / .480$ & 1 & 110 & 210 & 1 & 0.6389 & 5.7500 & 1.000 \\
Wind_11 - Turbine_11 & $13.80 / .480$ & 1 & 111 & 211 & 1 & 0.6389 & 5.7500 & 1.000 \\
Wind_12 - Turbine_12 & $13.80 / .480$ & 1 & 112 & 212 & 1 & 0.6389 & 5.7500 & 1.000 \\
Wind_13 - Turbine_13 & $13.80 / .480$ & 1 & 113 & 213 & 1 & 0.6389 & 5.7500 & 1.000 \\
Wind_14 - Turbine_14 & $13.80 / .480$ & 1 & 114 & 214 & 1 & 0.6389 & 5.7500 & 1.000 \\
Wind_15 - Turbine_15 & $13.80 / .480$ & 1 & 115 & 215 & 1 & 0.6389 & 5.7500 & 1.000 \\
Wind_16 - Turbine_16 & $13.80 / .480$ & 1 & 116 & 216 & 1 & 0.6389 & 5.7500 & 1.000 \\
Wind_17 - Turbine_17 & $13.80 / .480$ & 1 & 117 & 217 & 1 & 0.6389 & 5.7500 & 1.000 \\
Wind_18 - Turbine_18 & $13.80 / .480$ & 1 & 118 & 218 & 1 & 0.6389 & 5.7500 & 1.000 \\
Wind_19 - Turbine_19 & $13.80 / .480$ & 1 & 119 & 219 & 1 & 0.6389 & 5.7500 & 1.000 \\
Wind_20 - Turbine_20 & $13.80 / .480$ & 1 & 120 & 220 & 1 & 0.6389 & 5.7500 & 1.000 \\
Wind_21 - Turbine_21 & $13.80 / .480$ & 1 & 121 & 221 & 1 & 0.6389 & 5.7500 & 1.000 \\
Wind_22 - Turbine_22 & $13.80 / .480$ & 1 & 122 & 222 & 1 & 0.6389 & 5.7500 & 1.000
\end{tabular}


Table B1.3

Transformer Characteristics

Plan A 750 - 15 kV

WindPACT Turbine Design Scaling Studies

Transformer Characteristics

Transf. Model Data

\begin{tabular}{|c|c|c|c|c|c|c|c|}
\hline \multirow[b]{4}{*}{ Transformer } & \multicolumn{3}{|c|}{ Transformer Characteristics } & \multicolumn{4}{|c|}{ Transf. Model Data } \\
\hline & & & & & stem & ase MVA & 100 \\
\hline & & Base & Top & & & Imped. & t. Base) \\
\hline & Voltage kV & MVA & Rating & $Z \%$ & $\mathbf{X} / \mathbf{R}$ & $\mathrm{R}(\mathrm{pu})$ & $X(\mathrm{pu})$ \\
\hline tation Transformer & $115 / 13.8$ & 30 & 50 & $8.5 \%$ & 19 & $0.014 \varsigma$ & 0.2833 \\
\hline Wind Mill Transforme & $13.80 / .480$ & 1 & 1 & $5.75 \%$ & 9 & $0.638 s$ & 5.7500 \\
\hline
\end{tabular}

\begin{tabular}{lcccccccc} 
& & Rating & Tap & To & Ckt. & \multicolumn{2}{c}{ Imped. (Syst. Base) } & \\
\cline { 1 - 1 } \cline { 6 - 7 } Transformer Name & Voltage kV & MVA & No. & No. & Id. & R(pu) & X(pu) & Tap \\
\hline Wind_23 - Turbine_23 & $13.80 / .480$ & 1 & 123 & 223 & 1 & 0.6389 & 5.7500 & 1.000 \\
Wind_24 - Turbine_24 & $13.80 / .480$ & 1 & 124 & 224 & 1 & 0.6389 & 5.7500 & 1.000 \\
Wind_25 - Turbine_25 & $13.80 / .480$ & 1 & 125 & 225 & 1 & 0.6389 & 5.7500 & 1.000 \\
Wind_26 - Turbine_26 & $13.80 / .480$ & 1 & 126 & 226 & 1 & 0.6389 & 5.7500 & 1.000 \\
Wind_27 - Turbine_27 & $13.80 / .480$ & 1 & 127 & 227 & 1 & 0.6389 & 5.7500 & 1.000
\end{tabular}


Table B2.1

Bus Description and Generator Characteristics

Plan A 750 - 25 kV

WindPACT Turbine Design Scaling Studies

$\begin{array}{cr}\text { Collector System } & \begin{array}{r}\text { Voltage }= \\ \text { Base } \\ \text { kV }\end{array} \\ \text { Bus No. Name } & \begin{array}{c}115.00 \\ 1 \text { Mission_115 }\end{array} \\ 2 \text { Mission_25 } & 24.9 \\ 10 \text { Riser_A } & 24.9 \\ \text { 15 Riser_B } & 24.9 \\ \text { 20 Riser_C } & 24.9 \\ 101 \text { Wind_01 } & 24.9 \\ 102 \text { Wind_02 } & 24.9 \\ 103 \text { Wind_03 } & 24.9 \\ 104 \text { Wind_04 } & 24.9 \\ 105 \text { Wind_05 } & 24.9 \\ 106 \text { Wind_06 } & 24.9 \\ 107 \text { Wind_07 } & 24.9 \\ 108 \text { Wind_08 } & 24.9 \\ 109 \text { Wind_09 } & 24.9 \\ 110 \text { Wind_10 } & 24.9 \\ 111 \text { Wind_11 } & 24.9 \\ 112 \text { Wind_12 } & 24.9 \\ 113 \text { Wind_13 } & 24.9 \\ 114 \text { Wind_14 } & 24.9 \\ 115 \text { Wind_15 } & 24.9 \\ 116 \text { Wind_16 } & 24.9 \\ 117 \text { Wind_17 } & 24.9 \\ 118 \text { Wind_18 } & 24.9 \\ 119 \text { Wind_19 } & 24.9 \\ 120 \text { Wind_20 } & 24.9 \\ 121 \text { Wind_21 } & 24.9 \\ 122 \text { Wind_22 } & 24.9\end{array}$

24.9

Pgen

MW

MVAR

Qmin

$\begin{array}{cc}\text { Qmax } & \text { Qmin } \\ \text { MVAR } & \text { MVAR }\end{array}$

Power Flow Model

\begin{tabular}{cc} 
Vhold & \multicolumn{2}{c}{ Bus Type } \\
BType & BT \\
Reference & 3 \\
Load & 0 \\
Load & 0 \\
Load & 0 \\
Load & 0 \\
Load & 0 \\
Load & 0 \\
Load & 0 \\
Load & 0 \\
Load & 0 \\
Load & 0 \\
Load & 0 \\
Load & 0 \\
Load & 0 \\
Load & 0 \\
Load & 0 \\
Load & 0 \\
Load & 0 \\
Load & 0 \\
Load & 0 \\
Load & 0 \\
Load & 0 \\
Load & 0 \\
Load & 0 \\
Load & 0 \\
Load & 0 \\
Load & 0 \\
&
\end{tabular}

\author{
SC Model \\ X"d B(pu) \\ $0.0100 \quad 100.0000$
}


Table B2.1

Bus Description and Generator Characteristics

Plan A 750 - 25 kV

WindPACT Turbine Design Scaling Studies

\begin{tabular}{|c|c|c|c|c|c|c|c|c|c|}
\hline Collector System & Itage = & 24.9 & & & ower Flc & Model & & & \\
\hline & Base & Pgen & Qmax & Qmin & & Bus & & $\mathbf{S}$ & \\
\hline Bus No. & kV & MW & MVAR & MVAR & Vhold & BType & BT & $X " d$ & $\mathrm{~B}(\mathrm{pu})$ \\
\hline 123 Wind_23 & 24.9 & & & & & Load & 0 & & \\
\hline 124 Wind_24 & 24.9 & & & & & Load & 0 & & \\
\hline 125 Wind_25 & 24.9 & & & & & Load & 0 & & \\
\hline 126 Wind 26 & 24.9 & & & & & Load & 0 & & \\
\hline 127 Wind 27 & 24.9 & & & & & Load & 0 & & \\
\hline 128 Wind_28 & 24.9 & & & & & Load & 0 & & \\
\hline 129 Wind 29 & 24.9 & & & & & Load & 0 & & \\
\hline 130 Wind_30 & 24.9 & & & & & Load & 0 & & \\
\hline 131 Wind_31 & 24.9 & & & & & Load & 0 & & \\
\hline 132 Wind_32 & 24.9 & & & & & Load & 0 & & \\
\hline 133 Wind_33 & 24.9 & & & & & Load & 0 & & \\
\hline 134 Wind_34 & 24.9 & & & & & Load & 0 & & \\
\hline 135 Wind_35 & 24.9 & & & & & Load & 0 & & \\
\hline 136 Wind_36 & 24.9 & & & & & Load & 0 & & \\
\hline 137 Wind_37 & 24.9 & & & & & Load & 0 & & \\
\hline 138 Wind_38 & 24.9 & & & & & Load & 0 & & \\
\hline 139 Wind_39 & 24.9 & & & & & Load & 0 & & \\
\hline 140 Wind_40 & 24.9 & & & & & Load & 0 & & \\
\hline 141 Wind_41 & 24.9 & & & & & Load & 0 & & \\
\hline 142 Wind_42 & 24.9 & & & & & Load & 0 & & \\
\hline 143 Wind_43 & 24.9 & & & & & Load & 0 & & \\
\hline 144 Wind_44 & 24.9 & & & & & Load & 0 & & \\
\hline 145 Wind_45 & 24.9 & & & & & Load & 0 & & \\
\hline 146 Wind_46 & 24.9 & & & & & Load & 0 & & \\
\hline 147 Wind_47 & 24.9 & & & & & Load & 0 & & \\
\hline 148 Wind_48 & 24.9 & & & & & Load & 0 & & \\
\hline 149 Wind $^{4} 49$ & 24.9 & & & & & Load & 0 & & \\
\hline
\end{tabular}


Table B2.1

Bus Description and Generator Characteristics

Plan A 750 - 25 kV

WindPACT Turbine Design Scaling Studies

\begin{tabular}{|c|c|c|}
\hline \multicolumn{3}{|c|}{ Collector System Voltage $=$} \\
\hline Bus No. & Name & kV \\
\hline & ind_50 & 24.9 \\
\hline & ind_51 & 24.9 \\
\hline & ind 52 & 24.9 \\
\hline & ind 53 & 24.9 \\
\hline & ind 54 & 24.9 \\
\hline & ind 55 & 24.9 \\
\hline 15 & ind 56 & 24.9 \\
\hline 15 & ind_57 & 24.9 \\
\hline 15 & ind_58 & 24.9 \\
\hline 15 & ind_59 & 24.9 \\
\hline 16 & ind_60 & 24.9 \\
\hline & ind_61 & 24.9 \\
\hline & ind_62 & 24.9 \\
\hline & ind 63 & 24.9 \\
\hline & ind 64 & 24.9 \\
\hline & ind_65 & 24.9 \\
\hline & ind 66 & 24.9 \\
\hline
\end{tabular}

24.9

Qmax Qmin

Power Flow Model

Pgen

MVAR

MVAR

Bus Type

BType

Load

BT

Load

Load

Load

Load

Load

Load

Load

Load

Load

Load

Load

Load

Load

Load

Load

Load

SC Model X"d B(pu) 
Table B2.1

Bus Description and Generator Characteristics

Plan A 750 - 25 kV

WindPACT Turbine Design Scaling Studies

\begin{tabular}{|c|c|c|c|c|c|c|c|c|c|}
\hline & & Syst & n Base = & 100 & MVA & & & nort Circui & Model \\
\hline & & & & Data fo & Power Flc & w Model & & Machine & System \\
\hline & Mach & e Base & pf $=$ & -0.95 & 0.95 & & & Base & Base \\
\hline & kVA & kV & Pgen & Qmax & Qmin & Vhold & & X"d & X"d \\
\hline Windmill Model & 833 & 0.48 & 0.75 & 0.246513 & -0.246513 & 1.0000 & & 0.107 & 12.8451 \\
\hline System Equivalent & & 115.00 & & & & & & & 0.0100 \\
\hline & Base & Pgen & Qmax & $\begin{array}{l}\text { Power F } \\
\text { Qmin }\end{array}$ & low Model & Bus & & SC M & del \\
\hline Bus No. & kV & MW & MVAR & MVAR & Vhold & BType & BT & X"d & $B(p u)$ \\
\hline 201 Turbine_01 & 0.48 & 0.75 & 0.24651 & -0.246513 & 1.0000 & V-Control & 2 & 12.8451 & 0.0779 \\
\hline 202 Turbine_02 & 0.48 & 0.75 & 0.24651 & -0.246513 & 1.0000 & V-Control & 2 & 12.8451 & 0.0779 \\
\hline 203 Turbine_03 & 0.48 & 0.75 & 0.24651 & -0.246513 & 1.0000 & V-Control & 2 & 12.8451 & 0.0779 \\
\hline 204 Turbine_04 & 0.48 & 0.75 & 0.24651 & -0.246513 & 1.0000 & V-Control & 2 & 12.8451 & 0.0779 \\
\hline 205 Turbine_05 & 0.48 & 0.75 & 0.24651 & -0.246513 & 1.0000 & V-Control & 2 & 12.8451 & 0.0779 \\
\hline 206 Turbine_06 & 0.48 & 0.75 & 0.24651 & -0.246513 & 1.0000 & V-Control & 2 & 12.8451 & 0.0779 \\
\hline 207 Turbine_07 & 0.48 & 0.75 & 0.24651 & -0.246513 & 1.0000 & V-Control & 2 & 12.8451 & 0.0779 \\
\hline 208 Turbine_08 & 0.48 & 0.75 & 0.24651 & -0.246513 & 1.0000 & V-Control & 2 & 12.8451 & 0.0779 \\
\hline 209 Turbine_09 & 0.48 & 0.75 & 0.24651 & -0.246513 & 1.0000 & V-Control & 2 & 12.8451 & 0.0779 \\
\hline 210 Turbine_10 & 0.48 & 0.75 & 0.24651 & -0.246513 & 1.0000 & V-Control & 2 & 12.8451 & 0.0779 \\
\hline 211 Turbine_11 & 0.48 & 0.75 & 0.24651 & -0.246513 & 1.0000 & V-Control & 2 & 12.8451 & 0.0779 \\
\hline 212 Turbine_12 & 0.48 & 0.75 & 0.24651 & -0.246513 & 1.0000 & V-Control & 2 & 12.8451 & 0.0779 \\
\hline 213 Turbine_13 & 0.48 & 0.75 & 0.24651 & -0.246513 & 1.0000 & V-Control & 2 & 12.8451 & 0.0779 \\
\hline 214 Turbine_14 & 0.48 & 0.75 & 0.24651 & -0.246513 & 1.0000 & V-Control & 2 & 12.8451 & 0.0779 \\
\hline 215 Turbine_15 & 0.48 & 0.75 & 0.24651 & -0.246513 & 1.0000 & V-Control & 2 & 12.8451 & 0.0779 \\
\hline 216 Turbine_16 & 0.48 & 0.75 & 0.24651 & -0.246513 & 1.0000 & V-Control & 2 & 12.8451 & 0.0779 \\
\hline 217 Turbine_17 & 0.48 & 0.75 & 0.24651 & -0.246513 & 1.0000 & V-Control & 2 & 12.8451 & 0.0779 \\
\hline 218 Turbine_18 & 0.48 & 0.75 & 0.24651 & -0.246513 & 1.0000 & V-Control & 2 & 12.8451 & 0.0779 \\
\hline 219 Turbine_19 & 0.48 & 0.75 & 0.24651 & -0.246513 & 1.0000 & V-Control & 2 & 12.8451 & 0.0779 \\
\hline 220 Turbine_20 & 0.48 & 0.75 & 0.24651 & -0.246513 & 1.0000 & V-Control & 2 & 12.8451 & 0.0779 \\
\hline
\end{tabular}


Table B2.1

Bus Description and Generator Characteristics Plan A 750 - 25 kV

\section{WindPACT Turbine Design Scaling Studies}

\begin{tabular}{|c|c|c|c|c|c|c|c|}
\hline \multirow[b]{2}{*}{ Name } & \multirow{2}{*}{$\begin{array}{c}\text { Base } \\
\text { kV }\end{array}$} & \multirow{2}{*}{$\begin{array}{c}\text { Pgen } \\
\text { MW }\end{array}$} & \multirow{2}{*}{$\begin{array}{l}\text { Qmax } \\
\text { MVAR }\end{array}$} & $\begin{array}{l}\text { Power } \\
\text { Qmin }\end{array}$ & & \multicolumn{2}{|c|}{ Bus Type } \\
\hline & & & & MVAR & Vhold & BType & BT \\
\hline 221 Turbine_21 & 0.48 & 0.75 & 0.24651 & -0.246513 & 1.0000 & V-Control & \\
\hline 222 Turbine_22 & 0.48 & 0.75 & 0.24651 & -0.246513 & 1.0000 & V-Control & \\
\hline 223 Turbine_23 & 0.48 & 0.75 & 0.24651 & -0.246513 & 1.0000 & V-Control & \\
\hline 224 Turbine_24 & 0.48 & 0.75 & 0.24651 & -0.246513 & 1.0000 & V-Control & \\
\hline 225 Turbine_25 & 0.48 & 0.75 & 0.24651 & -0.246513 & 1.0000 & V-Control & \\
\hline 226 Turbine_26 & 0.48 & 0.75 & 0.24651 & -0.246513 & 1.0000 & V-Control & \\
\hline 227 Turbine_27 & 0.48 & 0.75 & 0.24651 & -0.246513 & 1.0000 & V-Control & \\
\hline 228 Turbine_28 & 0.48 & 0.75 & 0.24651 & -0.246513 & 1.0000 & V-Control & \\
\hline 229 Turbine 29 & 0.48 & 0.75 & 0.24651 & -0.246513 & 1.0000 & V-Control & \\
\hline 230 Turbine 30 & 0.48 & 0.75 & 0.24651 & -0.246513 & 1.0000 & V-Control & \\
\hline 231 Turbine 31 & 0.48 & 0.75 & 0.24651 & -0.246513 & 1.0000 & V-Control & \\
\hline 232 Turbine 32 & 0.48 & 0.75 & 0.24651 & -0.246513 & 1.0000 & V-Control & \\
\hline 233 Turbine_33 & 0.48 & 0.75 & 0.24651 & -0.246513 & 1.0000 & V-Control & \\
\hline 234 Turbine_34 & 0.48 & 0.75 & 0.24651 & -0.246513 & 1.0000 & V-Control & \\
\hline 235 Turbine_35 & 0.48 & 0.75 & 0.24651 & -0.246513 & 1.0000 & V-Control & \\
\hline 236 Turbine_36 & 0.48 & 0.75 & 0.24651 & -0.246513 & 1.0000 & V-Control & \\
\hline 237 Turbine_37 & 0.48 & 0.75 & 0.24651 & -0.246513 & 1.0000 & V-Control & \\
\hline 238 Turbine_38 & 0.48 & 0.75 & 0.24651 & -0.246513 & 1.0000 & V-Control & \\
\hline 239 Turbine_39 & 0.48 & 0.75 & 0.24651 & -0.246513 & 1.0000 & V-Control & \\
\hline 240 Turbine_40 & 0.48 & 0.75 & 0.24651 & -0.246513 & 1.0000 & V-Control & \\
\hline 241 Turbine 41 & 0.48 & 0.75 & 0.24651 & -0.246513 & 1.0000 & V-Control & \\
\hline 242 Turbine 42 & 0.48 & 0.75 & 0.24651 & -0.246513 & 1.0000 & V-Control & \\
\hline 243 Turbine 43 & 0.48 & 0.75 & 0.24651 & -0.246513 & 1.0000 & V-Control & \\
\hline 244 Turbine 44 & 0.48 & 0.75 & 0.24651 & -0.246513 & 1.0000 & V-Control & \\
\hline 245 Turbine_45 & 0.48 & 0.75 & 0.24651 & -0.246513 & 1.0000 & V-Control & \\
\hline 246 Turbine_46 & 0.48 & 0.75 & 0.24651 & -0.246513 & 1.0000 & V-Control & \\
\hline 247 Turbine_47 & 0.48 & 0.75 & 0.24651 & -0.246513 & 1.0000 & V-Control & \\
\hline
\end{tabular}

\begin{tabular}{|c|c|c|}
\hline & \multicolumn{2}{|c|}{. } \\
\hline & X"d & $B(p u)$ \\
\hline 2 & 12.8451 & 0.0779 \\
\hline 2 & 12.8451 & 0.0779 \\
\hline 2 & 12.8451 & 0.0779 \\
\hline 2 & 12.8451 & 0.0779 \\
\hline 2 & 12.8451 & 0.0779 \\
\hline 2 & 12.8451 & 0.0779 \\
\hline 2 & 12.8451 & 0.0779 \\
\hline 2 & 12.8451 & 0.0779 \\
\hline 2 & 12.8451 & 0.0779 \\
\hline 2 & 12.8451 & 0.0779 \\
\hline 2 & 12.8451 & 0.0779 \\
\hline 2 & 12.8451 & 0.0779 \\
\hline 2 & 12.8451 & 0.0779 \\
\hline 2 & 12.8451 & 0.0779 \\
\hline 2 & 12.8451 & 0.0779 \\
\hline 2 & 12.8451 & 0.0779 \\
\hline 2 & 12.8451 & 0.0779 \\
\hline 2 & 12.8451 & 0.0779 \\
\hline 2 & 12.8451 & 0.0779 \\
\hline 2 & 12.8451 & 0.0779 \\
\hline 2 & 12.8451 & 0.0779 \\
\hline 2 & 12.8451 & 0.0779 \\
\hline 2 & 12.8451 & 0.0779 \\
\hline 2 & 12.8451 & 0.0779 \\
\hline 2 & 12.8451 & 0.0779 \\
\hline 2 & 12.8451 & 0.0779 \\
\hline 2 & 12.8451 & 0.0779 \\
\hline
\end{tabular}


Table B2.1

Bus Description and Generator Characteristics

Plan A 750 - 25 kV

WindPACT Turbine Design Scaling Studies

\begin{tabular}{|c|c|c|c|c|c|c|c|c|c|}
\hline & \multirow{2}{*}{$\begin{array}{c}\text { Base } \\
\mathrm{kV}\end{array}$} & \multirow{2}{*}{$\begin{array}{c}\text { Pgen } \\
\text { MW }\end{array}$} & \multirow{2}{*}{$\begin{array}{l}\text { Qmax } \\
\text { MVAR }\end{array}$} & $\begin{array}{l}\text { Power F } \\
\text { Qmin }\end{array}$ & W iviouel & \multicolumn{2}{|c|}{ Bus Type } & \multicolumn{2}{|c|}{ SC Model } \\
\hline Bus No. & & & & MVAR & Vhold & BType & BT & X"d & $B(p u)$ \\
\hline 248 Turbine_48 & 0.48 & 0.75 & 0.24651 & -0.246513 & 1.0000 & V-Control & 2 & 12.8451 & 0.0779 \\
\hline 249 Turbine_49 & 0.48 & 0.75 & 0.24651 & -0.246513 & 1.0000 & V-Control & 2 & 12.8451 & 0.0779 \\
\hline 250 Turbine_50 & 0.48 & 0.75 & 0.24651 & -0.246513 & 1.0000 & V-Control & 2 & 12.8451 & 0.0779 \\
\hline 251 Turbine_51 & 0.48 & 0.75 & 0.24651 & -0.246513 & 1.0000 & V-Control & 2 & 12.8451 & 0.0779 \\
\hline 252 Turbine_52 & 0.48 & 0.75 & 0.24651 & -0.246513 & 1.0000 & V-Control & 2 & 12.8451 & 0.0779 \\
\hline 253 Turbine_53 & 0.48 & 0.75 & 0.24651 & -0.246513 & 1.0000 & V-Control & 2 & 12.8451 & 0.0779 \\
\hline 254 Turbine_54 & 0.48 & 0.75 & 0.24651 & -0.246513 & 1.0000 & V-Control & 2 & 12.8451 & 0.0779 \\
\hline 255 Turbine_55 & 0.48 & 0.75 & 0.24651 & -0.246513 & 1.0000 & V-Control & 2 & 12.8451 & 0.0779 \\
\hline 256 Turbine_56 & 0.48 & 0.75 & 0.24651 & -0.246513 & 1.0000 & V-Control & 2 & 12.8451 & 0.0779 \\
\hline 257 Turbine_57 & 0.48 & 0.75 & 0.24651 & -0.246513 & 1.0000 & V-Control & 2 & 12.8451 & 0.0779 \\
\hline 258 Turbine_58 & 0.48 & 0.75 & 0.24651 & -0.246513 & 1.0000 & V-Control & 2 & 12.8451 & 0.0779 \\
\hline 259 Turbine_59 & 0.48 & 0.75 & 0.24651 & -0.246513 & 1.0000 & V-Control & 2 & 12.8451 & 0.0779 \\
\hline 260 Turbine_60 & 0.48 & 0.75 & 0.24651 & -0.246513 & 1.0000 & V-Control & 2 & 12.8451 & 0.0779 \\
\hline 261 Turbine_61 & 0.48 & 0.75 & 0.24651 & -0.246513 & 1.0000 & V-Control & 2 & 12.8451 & 0.0779 \\
\hline 262 Turbine_62 & 0.48 & 0.75 & 0.24651 & -0.246513 & 1.0000 & V-Control & 2 & 12.8451 & 0.0779 \\
\hline 263 Turbine_63 & 0.48 & 0.75 & 0.24651 & -0.246513 & 1.0000 & V-Control & 2 & 12.8451 & 0.0779 \\
\hline 264 Turbine_64 & 0.48 & 0.75 & 0.24651 & -0.246513 & 1.0000 & V-Control & 2 & 12.8451 & 0.0779 \\
\hline 265 Turbine_65 & 0.48 & 0.75 & 0.24651 & -0.246513 & 1.0000 & V-Control & 2 & 12.8451 & 0.0779 \\
\hline 266 Turbine_66 & 0.48 & 0.75 & 0.24651 & -0.246513 & 1.0000 & V-Control & 2 & 12.8451 & 0.0779 \\
\hline
\end{tabular}


Table B2.2

Distribution Cable and Overhead Line Characteristics

Plan A 750 - 25 kV

WindPACT Turbine Design Scaling Studies

\begin{tabular}{|c|c|c|c|c|c|c|c|c|c|c|c|c|c|c|}
\hline \multirow{2}{*}{\multicolumn{2}{|c|}{$\begin{array}{c}\text { Construction } \\
\text { Type }\end{array}$}} & \multirow{2}{*}{$\begin{array}{c}\text { Conductor Size } \\
\text { AWG or kcmil }\end{array}$} & \multirow{2}{*}{$\begin{array}{c}\text { Rated } \\
\text { kV }\end{array}$} & ohms/ & $100 \mathrm{ft}$. & \multicolumn{2}{|c|}{$\begin{array}{r}\text { Base MVA= } \\
\text { Volt Base= } \\
\text { Z Base= }\end{array}$} & \multicolumn{2}{|c|}{$\begin{array}{c}100 \\
24.90 \\
6.200 \\
\text { PU/1000 ft. }\end{array}$} & \multirow{2}{*}{$\begin{array}{l}\text { Rating } \\
\text { Amps }\end{array}$} & \multicolumn{2}{|c|}{ ohms/1000 ft. } & \multicolumn{2}{|c|}{ PU/1000 ft. } \\
\hline & & & & $\mathbf{R}$ & $\mathbf{X}$ & $\mathrm{pf} / \mathrm{ft}$ & \multirow{2}{*}{$\begin{array}{c}\mathbf{R} \\
0.0342\end{array}$} & \multirow{2}{*}{$\frac{X}{0.0082}$} & \multirow{2}{*}{$\frac{\text { B }}{0.000122}$} & & R0 & $\mathrm{XO}$ & R0 & $\mathrm{XO}$ \\
\hline 1 & UG-1/0-25 & $1 / 0 \mathrm{Al}$ & 24.90 & 0.2120 & 0.0510 & 52 & & & & 212 & 0.549 & 0.263 & 0.089 & 0.042 \\
\hline 2 & UG-4/0-25 & $4 / 0 \mathrm{Al}$ & 24.90 & 0.1070 & 0.0460 & 64 & 0.0173 & 0.0074 & 0.000150 & 312 & 0.334 & 0.11 & 0.054 & 0.018 \\
\hline 3 & UG-500-25 & $500 \mathrm{Al}$ & 24.90 & 0.0480 & 0.0370 & 86 & 0.0077 & 0.0060 & 0.000201 & 395 & 0.250 & 0.172 & 0.040 & 0.028 \\
\hline 4 & UG-750-25 & $750 \mathrm{Al}$ & 24.90 & 0.0340 & 0.0350 & 101 & 0.0055 & 0.0056 & 0.000236 & 475 & 0.177 & 0.163 & 0.029 & 0.026 \\
\hline 5 & OH-SC-795 & 795 ACSR & & 0.0242 & 0.1135 & 3.86 & 0.0039 & 0.0183 & 0.000009 & 395 & 0.126 & 0.529 & 0.020 & 0.085 \\
\hline 6 & OH-DC2-795 & 796 ACSR & & 0.0242 & 0.1056 & 3.86 & 0.0039 & 0.0170 & 0.000009 & 395 & 0.126 & 0.492 & 0.020 & 0.079 \\
\hline
\end{tabular}

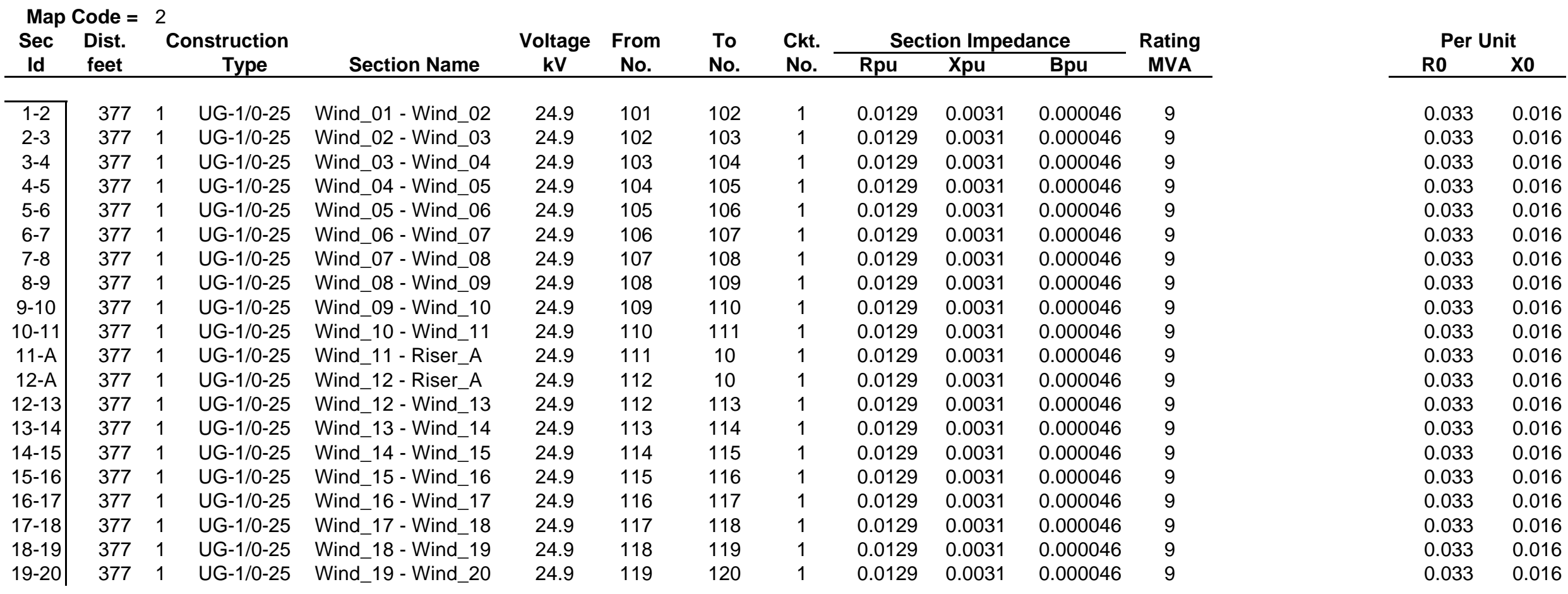


Table B2.2

Distribution Cable and Overhead Line Characteristics

Plan A 750 - 25 kV

WindPACT Turbine Design Scaling Studies

\begin{tabular}{|c|c|c|c|c|c|c|c|c|c|c|c|c|c|c|}
\hline \multirow{2}{*}{\multicolumn{2}{|c|}{$\begin{array}{c}\text { Construction } \\
\text { Type }\end{array}$}} & \multirow{2}{*}{$\begin{array}{l}\text { Conductor Size } \\
\text { AWG or kcmil }\end{array}$} & \multirow{2}{*}{$\begin{array}{c}\text { Rated } \\
\text { kV }\end{array}$} & ohms/1 & $00 \mathrm{ft}$. & \multicolumn{2}{|c|}{$\begin{array}{r}\text { Base MVA= } \\
\text { Volt Base= } \\
\text { Z Base= }\end{array}$} & \multicolumn{2}{|c|}{$\begin{array}{c}100 \\
24.90 \\
6.200 \\
\text { PU/1000 ft. }\end{array}$} & \multirow{2}{*}{$\begin{array}{l}\text { Rating } \\
\text { Amps }\end{array}$} & \multicolumn{2}{|c|}{ ohms/1000 ft. } & \multicolumn{2}{|c|}{ PU/1000 ft. } \\
\hline & & & & $\mathbf{R}$ & $X$ & $\mathrm{pf} / \mathrm{ft}$ & $\mathbf{R}$ & $\mathbf{X}$ & B & & R0 & $\mathrm{XO}$ & Ro & $\mathrm{XO}$ \\
\hline 1 & UG-1/0-25 & $1 / 0 \mathrm{Al}$ & 24.90 & 0.2120 & 0.0510 & 52 & 0.0342 & 0.0082 & 0.000122 & 212 & 0.549 & 0.263 & 0.089 & 0.042 \\
\hline 2 & UG-4/0-25 & $4 / 0 \mathrm{Al}$ & 24.90 & 0.1070 & 0.0460 & 64 & 0.0173 & 0.0074 & 0.000150 & 312 & 0.334 & 0.11 & 0.054 & 0.018 \\
\hline 3 & UG-500-25 & $500 \mathrm{Al}$ & 24.90 & 0.0480 & 0.0370 & 86 & 0.0077 & 0.0060 & 0.000201 & 395 & 0.250 & 0.172 & 0.040 & 0.028 \\
\hline 4 & UG-750-25 & $750 \mathrm{Al}$ & 24.90 & 0.0340 & 0.0350 & 101 & 0.0055 & 0.0056 & 0.000236 & 475 & 0.177 & 0.163 & 0.029 & 0.026 \\
\hline 5 & $\mathrm{OH}-\mathrm{SC}-795$ & 795 ACSR & & 0.0242 & 0.1135 & 3.86 & 0.0039 & 0.0183 & 0.000009 & 395 & 0.126 & 0.529 & 0.020 & 0.085 \\
\hline 6 & OH-DC2-795 & 796 ACSR & & 0.0242 & 0.1056 & 3.86 & 0.0039 & 0.0170 & 0.000009 & 395 & 0.126 & 0.492 & 0.020 & 0.079 \\
\hline
\end{tabular}

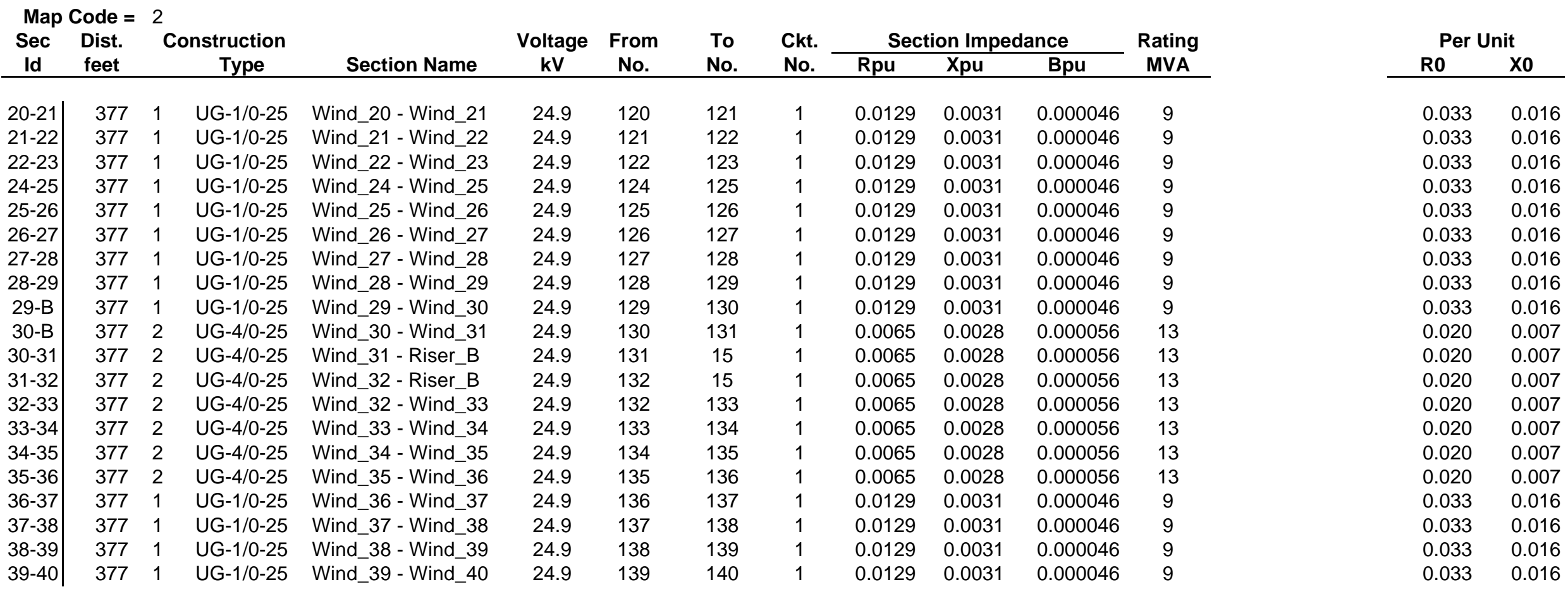


Table B2.2

Distribution Cable and Overhead Line Characteristics

Plan A 750 - 25 kV

WindPACT Turbine Design Scaling Studies

\begin{tabular}{|c|c|c|c|c|c|c|c|c|c|c|c|c|c|c|}
\hline \multirow{2}{*}{\multicolumn{2}{|c|}{$\begin{array}{c}\text { Construction } \\
\text { Type }\end{array}$}} & \multirow{2}{*}{$\begin{array}{l}\text { Conductor Size } \\
\text { AWG or kcmil }\end{array}$} & \multirow{2}{*}{$\begin{array}{c}\text { Rated } \\
\text { kV }\end{array}$} & ohms/1 & $00 \mathrm{ft}$. & \multicolumn{2}{|c|}{$\begin{array}{r}\text { Base MVA= } \\
\text { Volt Base= } \\
\text { Z Base= }\end{array}$} & \multicolumn{2}{|c|}{$\begin{array}{c}100 \\
24.90 \\
6.200 \\
\text { PU/1000 ft. }\end{array}$} & \multirow{2}{*}{$\begin{array}{l}\text { Rating } \\
\text { Amps }\end{array}$} & \multicolumn{2}{|c|}{ ohms/1000 ft. } & \multicolumn{2}{|c|}{ PU/1000 ft. } \\
\hline & & & & $\mathbf{R}$ & $X$ & $\mathrm{pf} / \mathrm{ft}$ & $\mathbf{R}$ & $\mathbf{X}$ & B & & R0 & $\mathrm{XO}$ & Ro & $\mathrm{XO}$ \\
\hline 1 & UG-1/0-25 & $1 / 0 \mathrm{Al}$ & 24.90 & 0.2120 & 0.0510 & 52 & 0.0342 & 0.0082 & 0.000122 & 212 & 0.549 & 0.263 & 0.089 & 0.042 \\
\hline 2 & UG-4/0-25 & $4 / 0 \mathrm{Al}$ & 24.90 & 0.1070 & 0.0460 & 64 & 0.0173 & 0.0074 & 0.000150 & 312 & 0.334 & 0.11 & 0.054 & 0.018 \\
\hline 3 & UG-500-25 & $500 \mathrm{Al}$ & 24.90 & 0.0480 & 0.0370 & 86 & 0.0077 & 0.0060 & 0.000201 & 395 & 0.250 & 0.172 & 0.040 & 0.028 \\
\hline 4 & UG-750-25 & $750 \mathrm{Al}$ & 24.90 & 0.0340 & 0.0350 & 101 & 0.0055 & 0.0056 & 0.000236 & 475 & 0.177 & 0.163 & 0.029 & 0.026 \\
\hline 5 & $\mathrm{OH}-\mathrm{SC}-795$ & 795 ACSR & & 0.0242 & 0.1135 & 3.86 & 0.0039 & 0.0183 & 0.000009 & 395 & 0.126 & 0.529 & 0.020 & 0.085 \\
\hline 6 & OH-DC2-795 & 796 ACSR & & 0.0242 & 0.1056 & 3.86 & 0.0039 & 0.0170 & 0.000009 & 395 & 0.126 & 0.492 & 0.020 & 0.079 \\
\hline
\end{tabular}

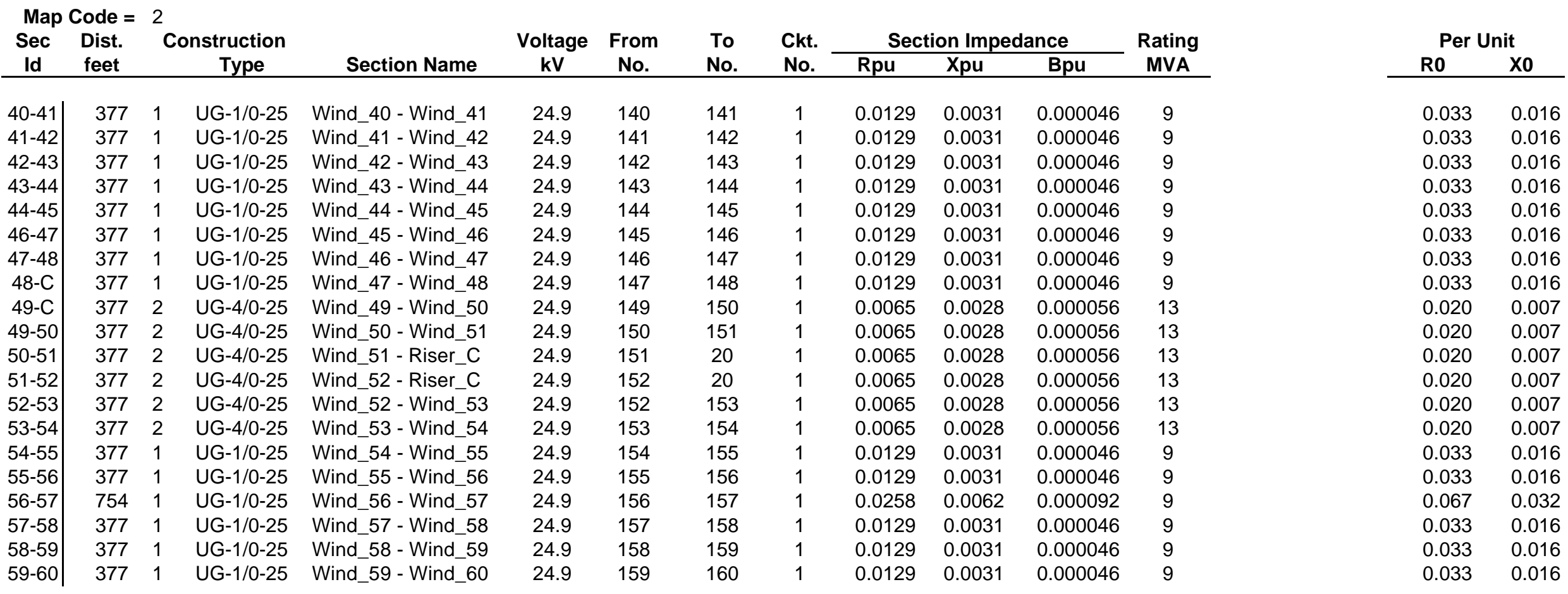


Table B2.2

Distribution Cable and Overhead Line Characteristics

Plan A 750 - 25 kV

WindPACT Turbine Design Scaling Studies

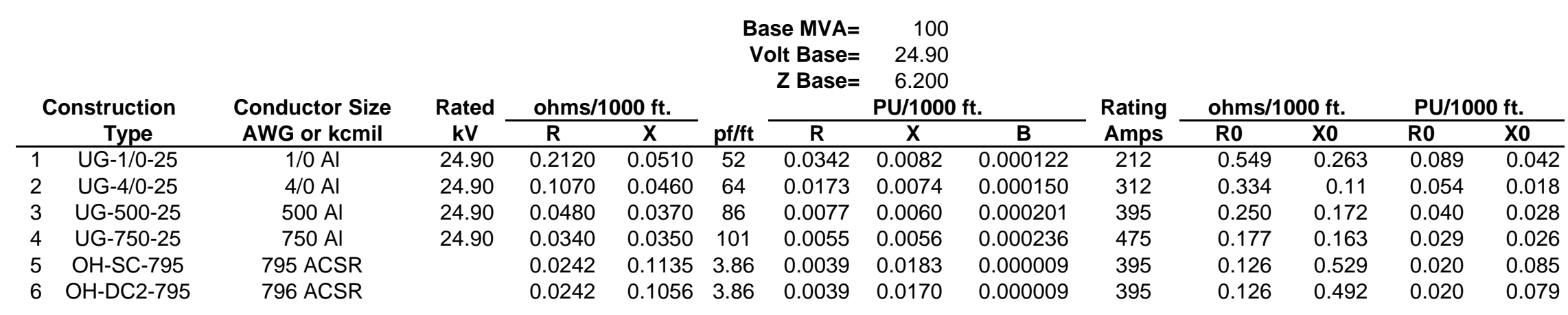

\begin{tabular}{|c|c|c|c|c|c|c|c|c|c|c|c|c|c|c|}
\hline \multicolumn{3}{|c|}{ Map Code $=2$} & \multicolumn{12}{|c|}{2} \\
\hline \multirow{2}{*}{$\begin{array}{l}\text { Sec } \\
\text { Id }\end{array}$} & \multirow{2}{*}{$\begin{array}{l}\text { Dist. } \\
\text { feet }\end{array}$} & \multirow{2}{*}{\multicolumn{2}{|c|}{$\begin{array}{c}\text { Construction } \\
\text { Type }\end{array}$}} & \multirow[b]{2}{*}{ Section Name } & \multirow{2}{*}{$\begin{array}{c}\text { Voltage } \\
\text { kV }\end{array}$} & \multirow{2}{*}{$\begin{array}{c}\text { From } \\
\text { No. }\end{array}$} & \multirow{2}{*}{$\begin{array}{l}\text { To } \\
\text { No. }\end{array}$} & \multirow{2}{*}{$\begin{array}{l}\text { Ckt. } \\
\text { No. }\end{array}$} & \multicolumn{3}{|c|}{ Section Impedance } & \multirow{2}{*}{$\begin{array}{c}\text { Rating } \\
\text { MVA }\end{array}$} & \multicolumn{2}{|c|}{ Per Unit } \\
\hline & & & & & & & & & Rpu & Xpu & Bpu & & Ro & XO \\
\hline 60-61 & 377 & 1 & UG-1/0-25 & Wind_60 - Wind_61 & 24.9 & 160 & 161 & 1 & 0.0129 & 0.0031 & 0.000046 & 9 & 0.033 & 0.016 \\
\hline 61-62 & 377 & 1 & UG-1/0-25 & Wind_61 - Wind_62 & 24.9 & 161 & 162 & 1 & 0.0129 & 0.0031 & 0.000046 & 9 & 0.033 & 0.016 \\
\hline $62-63$ & 377 & 1 & UG-1/0-25 & Wind_62 - Wind_63 & 24.9 & 162 & 163 & 1 & 0.0129 & 0.0031 & 0.000046 & 9 & 0.033 & 0.016 \\
\hline 63-64 & 754 & 1 & UG-1/0-25 & Wind_63 - Wind_64 & 24.9 & 163 & 164 & 1 & 0.0258 & 0.0062 & 0.000092 & 9 & 0.067 & 0.032 \\
\hline 64-65 & 377 & 1 & UG-1/0-25 & Wind_64 - Wind_65 & 24.9 & 164 & 165 & 1 & 0.0129 & 0.0031 & 0.000046 & 9 & 0.033 & 0.016 \\
\hline $65-66$ & 377 & 1 & UG-1/0-25 & Wind_65 - Wind_66 & 24.9 & 165 & 166 & 1 & 0.0129 & 0.0031 & 0.000046 & 9 & 0.033 & 0.016 \\
\hline
\end{tabular}


Table B2.2

Distribution Cable and Overhead Line Characteristics

Plan A 750 - 25 kV

WindPACT Turbine Design Scaling Studies

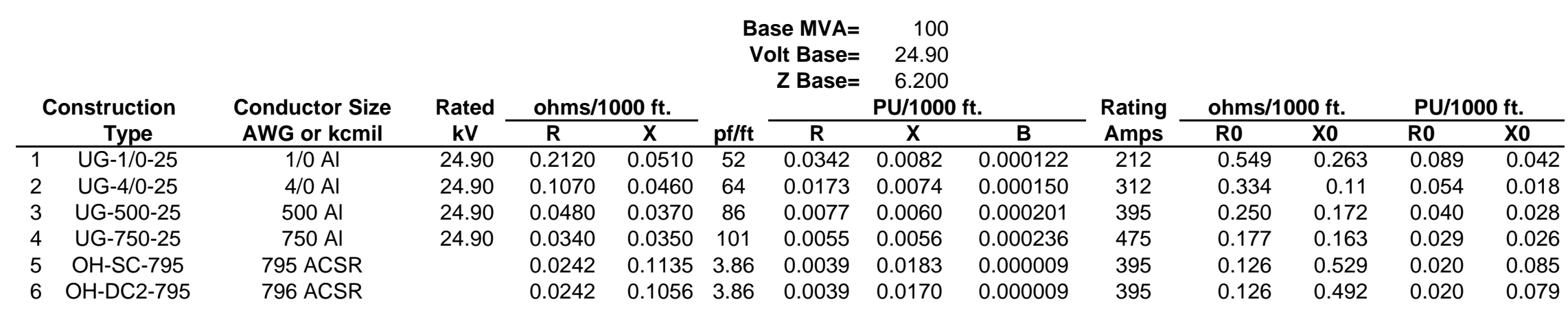

Map Code $=2$

\begin{tabular}{|c|c|c|c|c|c|c|c|c|c|c|c|c|c|c|}
\hline \multirow{2}{*}{$\begin{array}{l}\text { Sec } \\
\text { Id }\end{array}$} & \multirow{2}{*}{$\begin{array}{l}\text { Dist. } \\
\text { feet }\end{array}$} & \multirow{2}{*}{\multicolumn{2}{|c|}{$\begin{array}{c}\text { Construction } \\
\text { Type }\end{array}$}} & \multirow[b]{2}{*}{ Section Name } & \multirow{2}{*}{$\begin{array}{c}\text { Voltage } \\
\mathrm{kV}\end{array}$} & \multirow{2}{*}{$\begin{array}{c}\text { From } \\
\text { No. }\end{array}$} & \multirow{2}{*}{$\begin{array}{r}\text { To } \\
\text { No. }\end{array}$} & \multirow{2}{*}{$\begin{array}{l}\text { Ckt. } \\
\text { No. }\end{array}$} & \multicolumn{3}{|c|}{ Section Impedance } & \multirow{2}{*}{$\begin{array}{c}\text { Rating } \\
\text { MVA }\end{array}$} & \multicolumn{2}{|c|}{ Per Unit } \\
\hline & & & & & & & & & Rpu & Xpu & Bpu & & Ro & Xo \\
\hline$A-B$ & 2,300 & 5 & $\mathrm{OH}-\mathrm{SC}-795$ & Riser_A - Riser_B & 24.9 & 10 & 15 & 1 & 0.0090 & 0.0421 & 0.000021 & 17 & 0.047 & 0.196 \\
\hline$B-C$ & 2,300 & 5 & OH-SC-795 & Riser_B - Riser_C & 24.9 & 15 & 20 & 1 & 0.0090 & 0.0421 & 0.000021 & 17 & 0.047 & 0.196 \\
\hline $\mathrm{C}-\mathrm{F}$ & 1,000 & 5 & $\mathrm{OH}-\mathrm{SC}-795$ & Riser_C - Mission_25 & 24.9 & 20 & 2 & 1 & 0.0039 & 0.0183 & 0.000009 & 17 & 0.020 & 0.085 \\
\hline
\end{tabular}


Table B2.3

Transformer Characteristics

Plan A 750 - 25 kV

WindPACT Turbine Design Scaling System

Transformer Characteristics

Transf. Model Data

\begin{tabular}{|c|c|c|c|c|c|c|c|}
\hline \multirow[b]{4}{*}{ Transformer } & \multicolumn{3}{|c|}{ Transformer Characteristics } & \multicolumn{4}{|c|}{ Transf. Model Data } \\
\hline & & & & & stem & se MVA= & 100 \\
\hline & & Base & & & & Imped. ( & st. Base) \\
\hline & Voltage kV & MVA & Rating & $Z \%$ & $X / R$ & $\mathrm{R}(\mathrm{pu})$ & $X(\mathrm{pu})$ \\
\hline Station Transformer & $115 / 24.9$ & 30 & 50 & $8.5 \%$ & 19 & 0.0149 & 0.2833 \\
\hline Wind Mill Transformer & $24.90 / .480$ & 1 & 1 & $5.75 \%$ & 9 & 0.6389 & 5.7500 \\
\hline
\end{tabular}

\begin{tabular}{lcccccccc} 
& & Rating & Tap & To & Ckt. & \multicolumn{2}{c}{ Imped. (Syst. Base) } & \\
Transformer Name & Voltage kV & MVA & No. & No. & Id. & R(pu) & X(pu) & Tap \\
\hline Mission_115 - Mission_25 & $115 / 24.9$ & 50 & 1 & 2 & 1 & 0.0149 & 0.2833 & 1.000 \\
Wind_01 - Turbine_01 & $24.90 / .480$ & 1 & 101 & 201 & 1 & 0.6389 & 5.7500 & 1.000 \\
Wind_02 - Turbine_02 & $24.90 / .480$ & 1 & 102 & 202 & 1 & 0.6389 & 5.7500 & 1.000 \\
Wind_03 - Turbine_03 & $24.90 / .480$ & 1 & 103 & 203 & 1 & 0.6389 & 5.7500 & 1.000 \\
Wind_04 - Turbine_04 & $24.90 / .480$ & 1 & 104 & 204 & 1 & 0.6389 & 5.7500 & 1.000 \\
Wind_05 - Turbine_05 & $24.90 / .480$ & 1 & 105 & 205 & 1 & 0.6389 & 5.7500 & 1.000 \\
Wind_06 - Turbine_06 & $24.90 / .480$ & 1 & 106 & 206 & 1 & 0.6389 & 5.7500 & 1.000 \\
Wind_07 - Turbine_07 & $24.90 / .480$ & 1 & 107 & 207 & 1 & 0.6389 & 5.7500 & 1.000 \\
Wind_08 - Turbine_08 & $24.90 / .480$ & 1 & 108 & 208 & 1 & 0.6389 & 5.7500 & 1.000 \\
Wind_09 - Turbine_09 & $24.90 / .480$ & 1 & 109 & 209 & 1 & 0.6389 & 5.7500 & 1.000 \\
Wind_10 - Turbine_10 & $24.90 / .480$ & 1 & 110 & 210 & 1 & 0.6389 & 5.7500 & 1.000 \\
Wind_11 - Turbine_11 & $24.90 / .480$ & 1 & 111 & 211 & 1 & 0.6389 & 5.7500 & 1.000 \\
Wind_12 - Turbine_12 & $24.90 / .480$ & 1 & 112 & 212 & 1 & 0.6389 & 5.7500 & 1.000 \\
Wind_13 - Turbine_13 & $24.90 / .480$ & 1 & 113 & 213 & 1 & 0.6389 & 5.7500 & 1.000 \\
Wind_14 - Turbine_14 & $24.90 / .480$ & 1 & 114 & 214 & 1 & 0.6389 & 5.7500 & 1.000 \\
Wind_15 - Turbine_15 & $24.90 / .480$ & 1 & 115 & 215 & 1 & 0.6389 & 5.7500 & 1.000 \\
Wind_16 - Turbine_16 & $24.90 / .480$ & 1 & 116 & 216 & 1 & 0.6389 & 5.7500 & 1.000 \\
Wind_17 - Turbine_17 & $24.90 / .480$ & 1 & 117 & 217 & 1 & 0.6389 & 5.7500 & 1.000 \\
Wind_18 - Turbine_18 & $24.90 / .480$ & 1 & 118 & 218 & 1 & 0.6389 & 5.7500 & 1.000 \\
Wind_19 - Turbine_19 & $24.90 / .480$ & 1 & 119 & 219 & 1 & 0.6389 & 5.7500 & 1.000 \\
Wind_20 - Turbine_20 & $24.90 / .480$ & 1 & 120 & 220 & 1 & 0.6389 & 5.7500 & 1.000
\end{tabular}


Table B2.3

Transformer Characteristics

Plan A 750 - 25 kV

WindPACT Turbine Design Scaling System

Transformer Characteristics

Transf. Model Data

\begin{tabular}{|c|c|c|c|c|c|c|c|}
\hline \multirow[b]{4}{*}{ Transformer } & \multicolumn{3}{|c|}{ Transformer Characteristics } & \multicolumn{4}{|c|}{ Transf. Model Data } \\
\hline & & & & & stem & se MVA: & 100 \\
\hline & & Base & Tor & & & Imped. ( & t. Base) \\
\hline & Voltage kV & MVA & Rating & $Z \%$ & $\mathbf{X} / \mathbf{R}$ & $R(p u)$ & $X(p u)$ \\
\hline Station Transformer & $115 / 24.9$ & 30 & 50 & $8.5 \%$ & 19 & 0.0149 & 0.2833 \\
\hline Wind Mill Transformer & $24.90 / .480$ & 1 & 1 & $5.75 \%$ & 9 & 0.6389 & 5.7500 \\
\hline
\end{tabular}

\begin{tabular}{|c|c|c|c|c|c|c|c|c|}
\hline \multirow[b]{2}{*}{ Transformer Name } & \multirow[b]{2}{*}{ Voltage kV } & \multirow{2}{*}{$\begin{array}{c}\text { Rating } \\
\text { MVA }\end{array}$} & \multirow{2}{*}{$\begin{array}{l}\text { Tap } \\
\text { No. }\end{array}$} & \multirow{2}{*}{$\begin{array}{l}\text { To } \\
\text { No. }\end{array}$} & \multirow{2}{*}{$\begin{array}{l}\text { Ckt. } \\
\text { Id. }\end{array}$} & \multicolumn{2}{|c|}{ Imped. (Syst. Base) } & \multirow[b]{2}{*}{ Tap } \\
\hline & & & & & & $R(p u)$ & $X(p u)$ & \\
\hline Wind_21 - Turbine_21 & $24.90 / .480$ & 1 & 121 & 221 & 1 & 0.6389 & 5.7500 & 1.000 \\
\hline Wind_22 - Turbine_22 & $24.90 / .480$ & 1 & 122 & 222 & 1 & 0.6389 & 5.7500 & 1.000 \\
\hline Wind_23 - Turbine_23 & $24.90 / .480$ & 1 & 123 & 223 & 1 & 0.6389 & 5.7500 & 1.000 \\
\hline Wind_24 - Turbine_24 & $24.90 / .480$ & 1 & 124 & 224 & 1 & 0.6389 & 5.7500 & 1.000 \\
\hline Wind 25 - Turbine 25 & $24.90 / .480$ & 1 & 125 & 225 & 1 & 0.6389 & 5.7500 & 1.000 \\
\hline Wind 26 - Turbine 26 & $24.90 / .480$ & 1 & 126 & 226 & 1 & 0.6389 & 5.7500 & 1.000 \\
\hline Wind 27 - Turbine 27 & $24.90 / .480$ & 1 & 127 & 227 & 1 & 0.6389 & 5.7500 & 1.000 \\
\hline
\end{tabular}


Table B3.1

Bus Description and Generator Characteristics Plan A 2,500 - 25 kV

WindPACT Turbine Design Scaling Studies

\begin{tabular}{cr}
\multicolumn{2}{c}{$\begin{array}{c}\text { Collector System } \\
\text { Voltage }= \\
\text { Base } \\
\text { Bus No. Name }\end{array}$} \\
1 Mission_115 & $\begin{array}{r}115.00 \\
2 \text { Mission_25 }\end{array}$ \\
10 Riser_A & 24.9 \\
15 Riser_B & 24.9 \\
101 Wind_01 & 24.9 \\
102 Wind_02 & 24.9 \\
103 Wind_03 & 24.9 \\
104 Wind_04 & 24.9 \\
105 Wind_05 & 24.9 \\
106 Wind_06 & 24.9 \\
107 Wind_07 & 24.9 \\
108 Wind_08 & 24.9 \\
109 Wind_09 & 24.9 \\
110 Wind_10 & 24.9 \\
111 Wind_11 & 24.9 \\
112 Wind_12 & 24.9 \\
113 Wind_13 & 24.9 \\
114 Wind_14 & 24.9 \\
115 Wind_15 & 24.9 \\
116 Wind_16 & 24.9 \\
117 Wind_17 & 24.9 \\
118 Wind_18 & 24.9 \\
119 Wind_19 & 24.9 \\
120 Wind_20 & 24.9 \\
& 24.9
\end{tabular}

Power Flow Model

24.9

Pgen

Qmax Qmin

Bus Type

Vhold BType BT

1.0000 Reference

BT

Load

Load

Load

Load

Load

Load

Load

Load

Load

Load

Load

Load

Load

Load

Load

Load

Load

Load

Load

Load

Load

Load

Load
SC Model X"d $\quad B(p u)$
$0.0100 \quad 100.0000$


Table B3.1

Bus Description and Generator Characteristics

Plan A 2,500 - 25 kV

WindPACT Turbine Design Scaling Studies

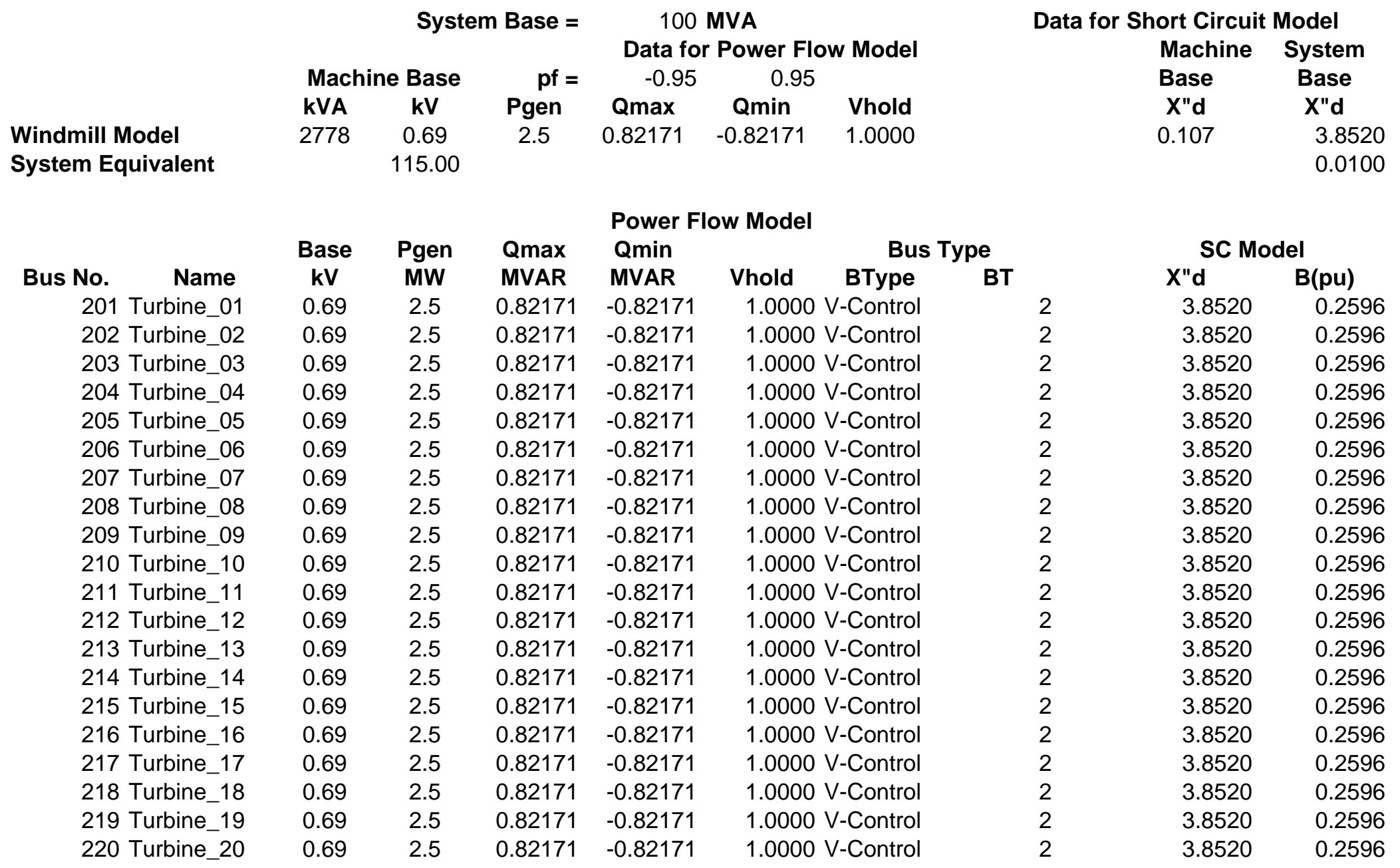


Table B3.2

Distribution Cable and Overhead Line Characteristics

Plan A 2,500 - 25 kV

WindPACT Turbine Design Scaling Studies

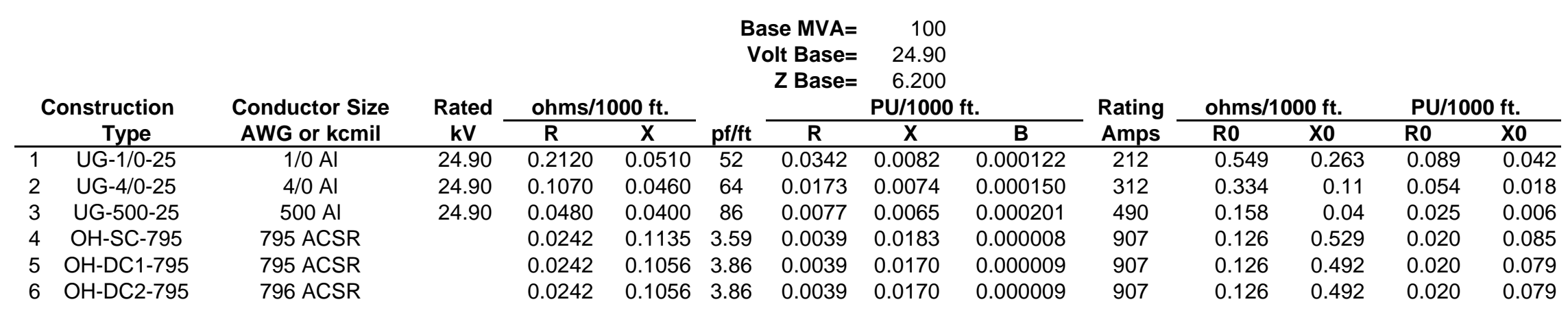

\begin{tabular}{|c|c|c|c|c|c|c|c|c|c|c|c|c|c|c|}
\hline \multirow{3}{*}{$\begin{array}{l}\text { Map } \\
\text { Sec } \\
\text { Id } \\
\end{array}$} & \multirow{3}{*}{$\begin{array}{c}\text { Code = } \\
\text { Dist. } \\
\text { feet }\end{array}$} & \multirow{3}{*}{\multicolumn{2}{|c|}{$\begin{array}{c}2 \\
\text { Construction } \\
\text { Type }\end{array}$}} & \multirow[b]{3}{*}{ Section Name } & \multirow{3}{*}{$\begin{array}{c}\text { Voltage } \\
\text { kV }\end{array}$} & \multirow{3}{*}{$\begin{array}{c}\text { From } \\
\text { No. }\end{array}$} & \multirow{3}{*}{$\begin{array}{r}\text { To } \\
\text { No. }\end{array}$} & \multirow{3}{*}{$\begin{array}{l}\text { Ckt. } \\
\text { No. }\end{array}$} & \multirow{2}{*}{\multicolumn{3}{|c|}{ Section Impedance }} & \multirow{3}{*}{$\begin{array}{c}\text { Rating } \\
\text { MVA }\end{array}$} & \multirow{2}{*}{\multicolumn{2}{|c|}{ Per Unit }} \\
\hline & & & & & & & & & & & & & & \\
\hline & & & & & & & & & Rpu & Xpu & Bpu & & Ro & XO \\
\hline $1-2$ & 642 & 1 & UG-1/0-25 & Wind_01 - Wind_02 & 24.9 & 101 & 102 & 1 & 0.0220 & 0.0053 & 0.000078 & 9 & 0.057 & 0.027 \\
\hline $2-3$ & 642 & 1 & UG-1/0-25 & Wind_02 - Wind_03 & 24.9 & 102 & 103 & 1 & 0.0220 & 0.0053 & 0.000078 & 9 & 0.057 & 0.027 \\
\hline $3-4$ & 642 & 1 & UG-1/0-25 & Wind_03 - Wind_04 & 24.9 & 103 & 104 & 1 & 0.0220 & 0.0053 & 0.000078 & 9 & 0.057 & 0.027 \\
\hline $4-5$ & 642 & 2 & UG-4/0-25 & Wind_04 - Wind_05 & 24.9 & 104 & 105 & 1 & 0.0111 & 0.0048 & 0.000096 & 13 & 0.035 & 0.011 \\
\hline $5-6$ & 642 & 2 & UG-4/0-25 & Wind_05 - Wind_06 & 24.9 & 105 & 106 & 1 & 0.0111 & 0.0048 & 0.000096 & 13 & 0.035 & 0.011 \\
\hline $6-A$ & 642 & 3 & UG-500-25 & Wind_06 - Riser_A & 24.9 & 106 & 10 & 1 & 0.0050 & 0.0041 & 0.000129 & 21 & 0.016 & 0.004 \\
\hline 7-A & 642 & 2 & UG-4/0-25 & Wind_07 - Riser_A & 24.9 & 107 & 10 & 1 & 0.0111 & 0.0048 & 0.000096 & 13 & 0.035 & 0.011 \\
\hline $7-8$ & 642 & 2 & UG-4/0-25 & Wind_07 - Wind_08 & 24.9 & 107 & 108 & 1 & 0.0111 & 0.0048 & 0.000096 & 13 & 0.035 & 0.011 \\
\hline $8-9$ & 642 & 1 & UG-1/0-25 & Wind_08 - Wind_09 & 24.9 & 108 & 109 & 1 & 0.0220 & 0.0053 & 0.000078 & 9 & 0.057 & 0.027 \\
\hline $9-10$ & 642 & 1 & UG-1/0-25 & Wind_09 - Wind_10 & 24.9 & 109 & 110 & 1 & 0.0220 & 0.0053 & 0.000078 & 9 & 0.057 & 0.027 \\
\hline $10-11$ & 642 & 1 & UG-1/0-25 & Wind_-10 - Wind_11 & 24.9 & 110 & 111 & 1 & 0.0220 & 0.0053 & 0.000078 & 9 & 0.057 & 0.027 \\
\hline $12-B$ & 642 & 2 & UG-4/0-25 & Wind_12 - Riser_B & 24.9 & 112 & 15 & 1 & 0.0111 & 0.0048 & 0.000096 & 13 & 0.035 & 0.011 \\
\hline $12-13$ & 642 & 2 & UG-4/0-25 & Wind_12 - Wind_13 & 24.9 & 112 & 113 & 1 & 0.0111 & 0.0048 & 0.000096 & 13 & 0.035 & 0.011 \\
\hline $13-14$ & 642 & 1 & UG-1/0-25 & Wind_13 - Wind_14 & 24.9 & 113 & 114 & 1 & 0.0220 & 0.0053 & 0.000078 & 9 & 0.057 & 0.027 \\
\hline $14-15$ & 642 & 1 & UG-1/0-25 & Wind_14 - Wind_15 & 24.9 & 114 & 115 & 1 & 0.0220 & 0.0053 & 0.000078 & 9 & 0.057 & 0.027 \\
\hline $15-16$ & 642 & 1 & UG-1/0-25 & Wind_15 - Wind_16 & 24.9 & 115 & 116 & 1 & 0.0220 & 0.0053 & 0.000078 & 9 & 0.057 & 0.027 \\
\hline $17-\mathrm{B}$ & 3,852 & 2 & UG-4/0-25 & Wind_17 - Riser_B & 24.9 & 117 & 15 & 1 & 0.0665 & 0.0286 & 0.000576 & 13 & 0.208 & 0.068 \\
\hline $17-18$ & 642 & 1 & UG-1/0-25 & Wind_17 - Wind_18 & 24.9 & 117 & 118 & 1 & 0.0220 & 0.0053 & 0.000078 & 9 & 0.057 & 0.027 \\
\hline $18-19$ & 642 & 1 & UG-1/0-25 & Wind_18 - Wind_19 & 24.9 & 118 & 119 & 1 & 0.0220 & 0.0053 & 0.000078 & 9 & 0.057 & 0.027 \\
\hline $19-20$ & 642 & 1 & UG-1/0-25 & Wind_19-Wind_20 & 24.9 & 119 & 120 & 1 & 0.0220 & 0.0053 & 0.000078 & 9 & 0.057 & 0.027 \\
\hline
\end{tabular}




\section{Distribution Cable and Overhead Line Characteristics \\ Plan A 2,500 - $25 \mathrm{kV}$}

WindPACT Turbine Design Scaling Studies

\begin{tabular}{|c|c|c|c|c|c|c|c|c|c|c|c|c|c|c|}
\hline \multirow{2}{*}{\multicolumn{2}{|c|}{$\begin{array}{c}\text { Construction } \\
\text { Type }\end{array}$}} & \multirow{2}{*}{$\begin{array}{c}\text { Conductor Size } \\
\text { AWG or kcmil }\end{array}$} & \multirow{2}{*}{$\begin{array}{c}\text { Rated } \\
\text { kV }\end{array}$} & ohms/1 & $00 \mathrm{ft}$. & \multicolumn{2}{|c|}{$\begin{array}{r}\text { Base MVA= } \\
\text { Volt Base= } \\
\text { Z Base }=\end{array}$} & \multicolumn{2}{|c|}{$\begin{array}{r}100 \\
24.90 \\
6.200 \\
U / 1000\end{array}$} & \multirow{2}{*}{$\begin{array}{l}\text { Rating } \\
\text { Amps }\end{array}$} & \multicolumn{2}{|c|}{ ohms/1000 ft. } & \multicolumn{2}{|c|}{ PU/1000 ft. } \\
\hline & & & & $\mathbf{R}$ & $\mathbf{X}$ & $\mathrm{pf} / \mathrm{ft}$ & $\mathbf{R}$ & $\mathbf{X}$ & \multirow{2}{*}{$\frac{\text { B }}{0.000122}$} & & Ro & $\mathbf{X O}$ & R0 & $\mathrm{XO}$ \\
\hline 1 & UG-1/0-25 & $1 / 0 \mathrm{Al}$ & 24.90 & 0.2120 & 0.0510 & 52 & 0.0342 & 0.0082 & & 212 & 0.549 & 0.263 & 0.089 & 0.042 \\
\hline 2 & UG-4/0-25 & 4/0 Al & 24.90 & 0.1070 & 0.0460 & 64 & 0.0173 & 0.0074 & 0.000150 & 312 & 0.334 & 0.11 & 0.054 & 0.018 \\
\hline 3 & UG-500-25 & $500 \mathrm{Al}$ & 24.90 & 0.0480 & 0.0400 & 86 & 0.0077 & 0.0065 & 0.000201 & 490 & 0.158 & 0.04 & 0.025 & 0.006 \\
\hline 4 & OH-SC-795 & 795 ACSR & & 0.0242 & 0.1135 & 3.59 & 0.0039 & 0.0183 & 0.000008 & 907 & 0.126 & 0.529 & 0.020 & 0.085 \\
\hline 5 & OH-DC1-795 & 795 ACSR & & 0.0242 & 0.1056 & 3.86 & 0.0039 & 0.0170 & 0.000009 & 907 & 0.126 & 0.492 & 0.020 & 0.079 \\
\hline 6 & OH-DC2-795 & 796 ACSR & & 0.0242 & 0.1056 & 3.86 & 0.0039 & 0.0170 & 0.000009 & 907 & 0.126 & 0.492 & 0.020 & 0.079 \\
\hline
\end{tabular}

Map Code $=2$

Sec Dist. Construction

Id feet Type

$\begin{array}{cccc} & \begin{array}{c}\text { Voltage } \\ \text { kV }\end{array} & \begin{array}{c}\text { From } \\ \text { No. }\end{array} & \text { To } \\ \text { So. }\end{array}$

Ckt.

Section Impedance

Bpu

Rating

MVA

Per Unit

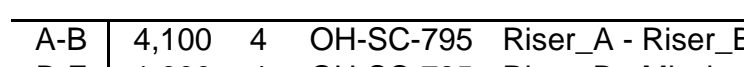

24.9

10

15

1

0.0160

0.0160
0.0062

0.0750

0.000034

39

39

0.083 XO

B-F $\quad 1,600 \quad 4$ OH-SC-795 Riser B-Mission 25

(2)


Table B3.3

Transformer Characteristics

Plan A 2500 - 25 kV

WindPACT Turbine Design Scaling Studies

Transformer Characteristics

Transf. Model Data

\begin{tabular}{|c|c|c|c|c|c|c|c|c|}
\hline & 1 & cteristic & & & & Transf. Mc & lel Data & \\
\hline & & & & & stem & ase MVA= & 100 & \\
\hline & & Base & To & & & Imped. (S & st. Base) & \\
\hline Transformer & Voltage kV & MVA & Rating & $Z \%$ & $\mathbf{X} / \mathbf{R}$ & $\mathrm{R}(\mathrm{pu})$ & $X(p u)$ & \\
\hline Station Transformer & $115 / 24.9$ & 30 & 50 & $8.5 \%$ & 19 & 0.0149 & 0.2833 & \\
\hline Wind Mill Transformer & $24.90 / .690$ & 3 & 3 & $5.50 \%$ & 9 & 0.2037 & 1.8333 & \\
\hline & & y & Tap & To & Ckt. & Imped. (s & st. Base) & \\
\hline Transformer Name & Voltage kV & MVA & No. & No. & Id. & $R(p u)$ & $\overline{X(p u)}$ & Tap \\
\hline Mission_115 - Mission_25 & $115 / 13.8$ & 50 & 1 & 2 & 1 & 0.0149 & 0.2833 & 1.000 \\
\hline Wind_01 - Turbine_01 & $24.90 / .690$ & 3 & 101 & 201 & 1 & 0.2037 & 1.8333 & 1.000 \\
\hline Wind_02 - Turbine_02 & $24.90 / .690$ & 3 & 102 & 202 & 1 & 0.2037 & 1.8333 & 1.000 \\
\hline Wind_03 - Turbine_03 & $24.90 / .690$ & 3 & 103 & 203 & 1 & 0.2037 & 1.8333 & 1.000 \\
\hline Wind_04 - Turbine_04 & $24.90 / .690$ & 3 & 104 & 204 & 1 & 0.2037 & 1.8333 & 1.000 \\
\hline Wind_05 - Turbine_05 & $24.90 / .690$ & 3 & 105 & 205 & 1 & 0.2037 & 1.8333 & 1.000 \\
\hline Wind_06 - Turbine_06 & $24.90 / .690$ & 3 & 106 & 206 & 1 & 0.2037 & 1.8333 & 1.000 \\
\hline Wind_07 - Turbine_07 & $24.90 / .690$ & 3 & 107 & 207 & 1 & 0.2037 & 1.8333 & 1.000 \\
\hline Wind_08 - Turbine_08 & $24.90 / .690$ & 3 & 108 & 208 & 1 & 0.2037 & 1.8333 & 1.000 \\
\hline Wind_09 - Turbine_09 & $24.90 / .690$ & 3 & 109 & 209 & 1 & 0.2037 & 1.8333 & 1.000 \\
\hline Wind_10 - Turbine_10 & $24.90 / .690$ & 3 & 110 & 210 & 1 & 0.2037 & 1.8333 & 1.000 \\
\hline Wind_11 - Turbine_11 & $24.90 / .690$ & 3 & 111 & 211 & 1 & 0.2037 & 1.8333 & 1.000 \\
\hline Wind_12 - Turbine_12 & $24.90 / .690$ & 3 & 112 & 212 & 1 & 0.2037 & 1.8333 & 1.000 \\
\hline Wind_13 - Turbine_13 & $24.90 / .690$ & 3 & 113 & 213 & 1 & 0.2037 & 1.8333 & 1.000 \\
\hline Wind_14 - Turbine_14 & $24.90 / .690$ & 3 & 114 & 214 & 1 & 0.2037 & 1.8333 & 1.000 \\
\hline Wind_15 - Turbine_15 & $24.90 / .690$ & 3 & 115 & 215 & 1 & 0.2037 & 1.8333 & 1.000 \\
\hline Wind_16 - Turbine_16 & $24.90 / .690$ & 3 & 116 & 216 & 1 & 0.2037 & 1.8333 & 1.000 \\
\hline Wind_17 - Turbine_17 & $24.90 / .690$ & 3 & 117 & 217 & 1 & 0.2037 & 1.8333 & 1.000 \\
\hline Wind_18 - Turbine_18 & $24.90 / .690$ & 3 & 118 & 218 & 1 & 0.2037 & 1.8333 & 1.000 \\
\hline Wind_19 - Turbine_19 & $24.90 / .690$ & 3 & 119 & 219 & 1 & 0.2037 & 1.8333 & 1.000 \\
\hline Wind_20 - Turbine_20 & $24.90 / .690$ & 3 & 120 & 220 & 1 & 0.2037 & 1.8333 & 1.000 \\
\hline
\end{tabular}


Table B4.1

Bus Description and Generator Characteristics Plan B 2,500 - 35 kV

WindPACT Turbine Design Scaling Studies

$\begin{array}{cr}\text { Collector System } & \begin{array}{r}\text { Voltage }= \\ \text { Base } \\ \text { kV }\end{array} \\ \text { Bus No. Name } & \begin{array}{r}115.00 \\ 1 \text { Mission_115 }\end{array} \\ 2 \text { Mission_35 } & 34.5 \\ 10 \text { Riser_A } & 34.5 \\ 15 \text { Riser_B } & 34.5 \\ 101 \text { Wind_01 } & 34.5 \\ 102 \text { Wind_02 } & 34.5 \\ 103 \text { Wind_03 } & 34.5 \\ 104 \text { Wind_04 } & 34.5 \\ 105 \text { Wind_05 } & 34.5 \\ 106 \text { Wind_06 } & 34.5 \\ 107 \text { Wind_07 } & 34.5 \\ 108 \text { Wind_08 } & 34.5 \\ 109 \text { Wind_09 } & 34.5 \\ 110 \text { Wind_10 } & 34.5 \\ 111 \text { Wind_11 } & 34.5 \\ 112 \text { Wind_12 } & 34.5 \\ 113 \text { Wind_13 } & 34.5 \\ 114 \text { Wind_14 } & 34.5 \\ 115 \text { Wind_15 } & 34.5 \\ 116 \text { Wind_16 } & 34.5 \\ 117 \text { Wind_17 } & 34.5 \\ 118 \text { Wind_18 } & 34.5 \\ 119 \text { Wind_19 } & 34.5 \\ 120 \text { Wind_20 } & 34.5\end{array}$

34.5

Pgen

MW

Qmax

Qmin

Bus Type

Vhold

BType

BT

1.0000 Reference

Load

Load

Load

Load

Load

Load

Load

Load

Load

Load

Load

Load

Load

Load

Load

Load

Load

Load

Load

Load

Load

Load

Load
Power Flow Model
SC Model
X"d $\mathrm{B}(\mathrm{pu})$

3
0


Table B4.1

Bus Description and Generator Characteristics

Plan B 2,500 - 35 kV

WindPACT Turbine Design Scaling Studies

\begin{tabular}{|c|c|c|c|c|c|c|c|c|c|}
\hline & & $\mathrm{s}$ & n Bas & 100 & MVA & & & ort Circe & Model \\
\hline & & & & Data fo & Power Flc & w Model & & Machine & System \\
\hline & Mac & ne Base & pf $=$ & -0.95 & 0.95 & & & Base & Base \\
\hline & kVA & kV & Pgen & Qmax & Qmin & Vhold & & $X " d$ & $X " d$ \\
\hline Iindmill Model & 2778 & 0.69 & 2.5 & 0.82171 & -0.82171 & 1.0000 & & 0.107 & 3.8520 \\
\hline System Equivalent & & 115.00 & & & & & & & 0.0100 \\
\hline & Base & Pgen & Qmax & $\begin{array}{l}\text { Power F } \\
\text { Qmin }\end{array}$ & ow Model & Bus & & SC Mo & \\
\hline Bus No. & kV & MW & MVAR & MVAR & Vhold & BType & BT & X"d & $\mathrm{B}(\mathrm{pu})$ \\
\hline 201 Turbine_01 & 0.69 & 2.5 & 0.82171 & -0.82171 & 1.0000 & V-Control & 2 & 3.8520 & 0.2596 \\
\hline 202 Turbine_02 & 0.69 & 2.5 & 0.82171 & -0.82171 & 1.0000 & V-Control & 2 & 3.8520 & 0.2596 \\
\hline 203 Turbine_03 & 0.69 & 2.5 & 0.82171 & -0.82171 & 1.0000 & V-Control & 2 & 3.8520 & 0.2596 \\
\hline 204 Turbine_04 & 0.69 & 2.5 & 0.82171 & -0.82171 & 1.0000 & V-Control & 2 & 3.8520 & 0.2596 \\
\hline 205 Turbine_05 & 0.69 & 2.5 & 0.82171 & -0.82171 & 1.0000 & V-Control & 2 & 3.8520 & 0.2596 \\
\hline 206 Turbine_06 & 0.69 & 2.5 & 0.82171 & -0.82171 & 1.0000 & V-Control & 2 & 3.8520 & 0.2596 \\
\hline 207 Turbine_07 & 0.69 & 2.5 & 0.82171 & -0.82171 & 1.0000 & V-Control & 2 & 3.8520 & 0.2596 \\
\hline 208 Turbine_08 & 0.69 & 2.5 & 0.82171 & -0.82171 & 1.0000 & V-Control & 2 & 3.8520 & 0.2596 \\
\hline 209 Turbine_09 & 0.69 & 2.5 & 0.82171 & -0.82171 & 1.0000 & V-Control & 2 & 3.8520 & 0.2596 \\
\hline 210 Turbine_10 & 0.69 & 2.5 & 0.82171 & -0.82171 & 1.0000 & V-Control & 2 & 3.8520 & 0.2596 \\
\hline 211 Turbine_11 & 0.69 & 2.5 & 0.82171 & -0.82171 & 1.0000 & V-Control & 2 & 3.8520 & 0.2596 \\
\hline 212 Turbine_12 & 0.69 & 2.5 & 0.82171 & -0.82171 & 1.0000 & V-Control & 2 & 3.8520 & 0.2596 \\
\hline 213 Turbine_13 & 0.69 & 2.5 & 0.82171 & -0.82171 & 1.0000 & V-Control & 2 & 3.8520 & 0.2596 \\
\hline 214 Turbine_14 & 0.69 & 2.5 & 0.82171 & -0.82171 & 1.0000 & V-Control & 2 & 3.8520 & 0.2596 \\
\hline 215 Turbine_15 & 0.69 & 2.5 & 0.82171 & -0.82171 & 1.0000 & V-Control & 2 & 3.8520 & 0.2596 \\
\hline 216 Turbine_16 & 0.69 & 2.5 & 0.82171 & -0.82171 & 1.0000 & V-Control & 2 & 3.8520 & 0.2596 \\
\hline 217 Turbine_17 & 0.69 & 2.5 & 0.82171 & -0.82171 & 1.0000 & V-Control & 2 & 3.8520 & 0.2596 \\
\hline 218 Turbine_18 & 0.69 & 2.5 & 0.82171 & -0.82171 & 1.0000 & V-Control & 2 & 3.8520 & 0.2596 \\
\hline 219 Turbine_19 & 0.69 & 2.5 & 0.82171 & -0.82171 & 1.0000 & V-Control & 2 & 3.8520 & 0.2596 \\
\hline 220 Turbine_20 & 0.69 & 2.5 & 0.82171 & -0.82171 & 1.0000 & V-Control & 2 & 3.8520 & 0.2596 \\
\hline
\end{tabular}


Table B4.2

Distribution Cable and Overhead Line Characteristics

Plan B 2,500 - 35 kV

WindPACT Turbine Design Scaling Studies

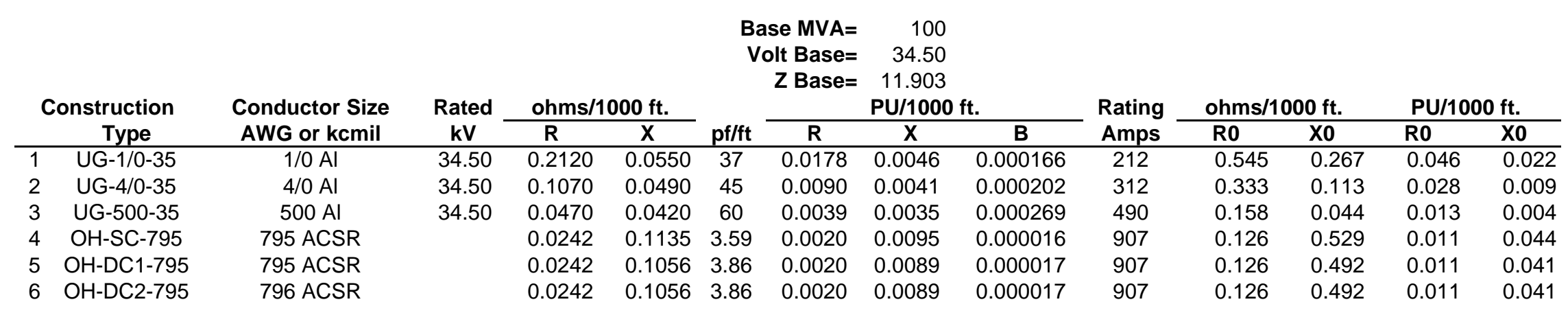

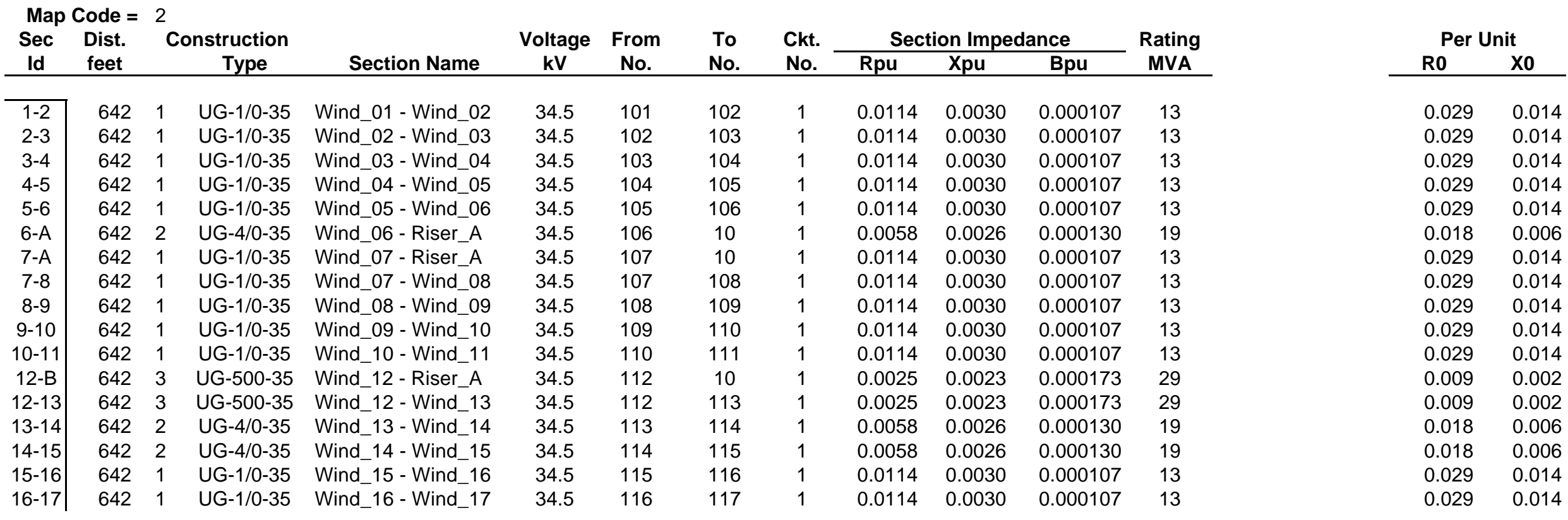


Table B4.2

Distribution Cable and Overhead Line Characteristics

Plan B 2,500 - 35 kV

WindPACT Turbine Design Scaling Studies

\begin{tabular}{|c|c|c|c|c|c|c|c|c|c|c|c|c|c|c|}
\hline \multirow{2}{*}{\multicolumn{2}{|c|}{$\begin{array}{c}\text { Construction } \\
\text { Type }\end{array}$}} & \multirow{2}{*}{$\begin{array}{c}\text { Conductor Size } \\
\text { AWG or kcmil }\end{array}$} & \multirow{2}{*}{$\begin{array}{c}\text { Rated } \\
\text { kV }\end{array}$} & ohms/1 & $00 \mathrm{ft}$. & \multicolumn{2}{|c|}{$\begin{array}{r}\text { Base MVA= } \\
\text { Volt Base= } \\
\text { Z Base= }\end{array}$} & \multicolumn{2}{|c|}{$\begin{array}{c}100 \\
34.50 \\
11.903 \\
\text { PU/1000 ft. }\end{array}$} & \multirow{2}{*}{$\begin{array}{l}\text { Rating } \\
\text { Amps }\end{array}$} & \multicolumn{2}{|c|}{ ohms/1000 ft. } & \multicolumn{2}{|c|}{ PU/1000 ft. } \\
\hline & & & & $\mathbf{R}$ & $\mathbf{X}$ & $\mathrm{pf} / \mathrm{ft}$ & \multirow{2}{*}{$\begin{array}{c}\mathbf{R} \\
0.0178\end{array}$} & \multirow{2}{*}{$\frac{X}{0.0046}$} & \multirow{2}{*}{$\begin{array}{c}\text { B } \\
0.000166\end{array}$} & & R0 & $\mathrm{XO}$ & R0 & $\mathrm{XO}$ \\
\hline 1 & UG-1/0-35 & $1 / 0 \mathrm{Al}$ & 34.50 & 0.2120 & 0.0550 & 37 & & & & 212 & 0.545 & 0.267 & 0.046 & 0.022 \\
\hline 2 & UG-4/0-35 & $4 / 0 \mathrm{Al}$ & 34.50 & 0.1070 & 0.0490 & 45 & 0.0090 & 0.0041 & 0.000202 & 312 & 0.333 & 0.113 & 0.028 & 0.009 \\
\hline 3 & UG-500-35 & $500 \mathrm{Al}$ & 34.50 & 0.0470 & 0.0420 & 60 & 0.0039 & 0.0035 & 0.000269 & 490 & 0.158 & 0.044 & 0.013 & 0.004 \\
\hline 4 & OH-SC-795 & 795 ACSR & & 0.0242 & 0.1135 & 3.59 & 0.0020 & 0.0095 & 0.000016 & 907 & 0.126 & 0.529 & 0.011 & 0.044 \\
\hline 5 & $\mathrm{OH}-\mathrm{DC1}-795$ & 795 ACSR & & 0.0242 & 0.1056 & 3.86 & 0.0020 & 0.0089 & 0.000017 & 907 & 0.126 & 0.492 & 0.011 & 0.041 \\
\hline 6 & OH-DC2-795 & 796 ACSR & & 0.0242 & 0.1056 & 3.86 & 0.0020 & 0.0089 & 0.000017 & 907 & 0.126 & 0.492 & 0.011 & 0.041 \\
\hline
\end{tabular}

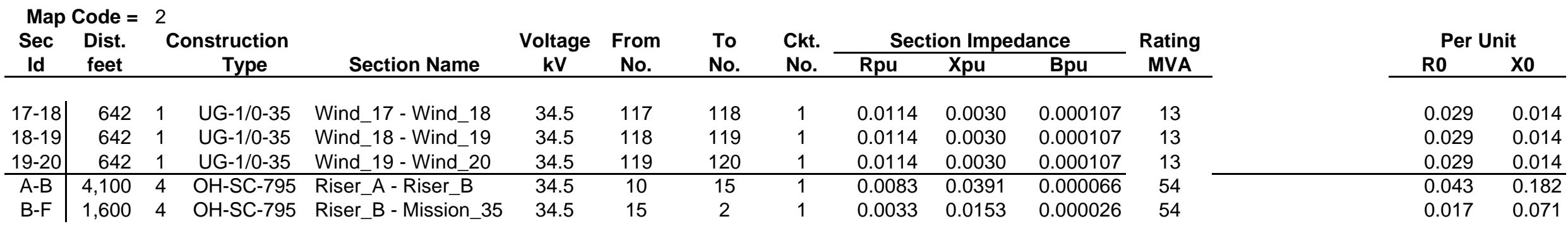




\section{Table B4.3 \\ Transformer Characteristics \\ Plan B 2,500 - 35 kV \\ WindPACT Turbine Design Scaling Studies}

\begin{tabular}{|c|c|c|c|c|c|c|c|}
\hline \multirow[b]{4}{*}{ Transformer } & \multicolumn{3}{|c|}{ Transformer Characteristics } & \multicolumn{4}{|c|}{ Transf. Model Data } \\
\hline & & & & & stem & ase MVA & 100 \\
\hline & & Base & Top & & & Imped. & t. Base) \\
\hline & Voltage kV & MVA & Rating & $Z \%$ & $\mathbf{X} / \mathbf{R}$ & $R(p u)$ & $X(p u)$ \\
\hline Station Transformer & $115 / 34.5$ & 30 & 50 & $8.5 \%$ & 19 & 0.0149 & 0.2833 \\
\hline Wind Mill Transformer & $34.50 / .690$ & 3 & 3 & $7.00 \%$ & 9 & 0.2593 & 2.3333 \\
\hline
\end{tabular}

\begin{tabular}{|c|c|c|c|c|c|c|c|c|}
\hline \multirow[b]{2}{*}{ Transformer Name } & \multirow[b]{2}{*}{ Voltage kV } & \multirow{2}{*}{$\begin{array}{c}\text { Rating } \\
\text { MVA }\end{array}$} & \multirow{2}{*}{$\begin{array}{l}\text { Tap } \\
\text { No. }\end{array}$} & \multirow{2}{*}{$\begin{array}{l}\text { To } \\
\text { No. }\end{array}$} & \multirow{2}{*}{$\begin{array}{l}\text { Ckt. } \\
\text { Id. }\end{array}$} & \multicolumn{2}{|c|}{ Imped. (Syst. Base) } & \multirow[b]{2}{*}{ Tap } \\
\hline & & & & & & $\mathbf{R}(\mathrm{pu})$ & $X(p u)$ & \\
\hline Mission_115 - Mission_35 & $115 / 13.8$ & 50 & 1 & 2 & 1 & 0.0149 & 0.2833 & 1.000 \\
\hline Wind $0 \overline{1}$ - Turbine 01 & $34.50 / .690$ & 3 & 101 & 201 & 1 & 0.2593 & 2.3333 & 1.000 \\
\hline Wind 02 - Turbine 02 & $34.50 / .690$ & 3 & 102 & 202 & 1 & 0.2593 & 2.3333 & 1.000 \\
\hline Wind 03 - Turbine 03 & $34.50 / .690$ & 3 & 103 & 203 & 1 & 0.2593 & 2.3333 & 1.000 \\
\hline Wind 04 - Turbine 04 & $34.50 / .690$ & 3 & 104 & 204 & 1 & 0.2593 & 2.3333 & 1.000 \\
\hline Wind 05 - Turbine 05 & $34.50 / .690$ & 3 & 105 & 205 & 1 & 0.2593 & 2.3333 & 1.000 \\
\hline Wind 06 - Turbine 06 & $34.50 / .690$ & 3 & 106 & 206 & 1 & 0.2593 & 2.3333 & 1.000 \\
\hline Wind 07 - Turbine 07 & $34.50 / .690$ & 3 & 107 & 207 & 1 & 0.2593 & 2.3333 & 1.000 \\
\hline Wind_08 - Turbine_08 & $34.50 / .690$ & 3 & 108 & 208 & 1 & 0.2593 & 2.3333 & 1.000 \\
\hline Wind_09 - Turbine_09 & $34.50 / .690$ & 3 & 109 & 209 & 1 & 0.2593 & 2.3333 & 1.000 \\
\hline Wind_10 - Turbine_10 & $34.50 / .690$ & 3 & 110 & 210 & 1 & 0.2593 & 2.3333 & 1.000 \\
\hline Wind_11 - Turbine_11 & $34.50 / .690$ & 3 & 111 & 211 & 1 & 0.2593 & 2.3333 & 1.000 \\
\hline Wind_12 - Turbine_12 & $34.50 / .690$ & 3 & 112 & 212 & 1 & 0.2593 & 2.3333 & 1.000 \\
\hline Wind_13 - Turbine_13 & $34.50 / .690$ & 3 & 113 & 213 & 1 & 0.2593 & 2.3333 & 1.000 \\
\hline Wind_14 - Turbine_14 & $34.50 / .690$ & 3 & 114 & 214 & 1 & 0.2593 & 2.3333 & 1.000 \\
\hline Wind_15 - Turbine_15 & $34.50 / .690$ & 3 & 115 & 215 & 1 & 0.2593 & 2.3333 & 1.000 \\
\hline Wind_16 - Turbine_16 & $34.50 / .690$ & 3 & 116 & 216 & 1 & 0.2593 & 2.3333 & 1.000 \\
\hline Wind_17 - Turbine_17 & $34.50 / .690$ & 3 & 117 & 217 & 1 & 0.2593 & 2.3333 & 1.000 \\
\hline Wind_18 - Turbine_18 & $34.50 / .690$ & 3 & 118 & 218 & 1 & 0.2593 & 2.3333 & 1.000 \\
\hline Wind_19 - Turbine_19 & $34.50 / .690$ & 3 & 119 & 219 & 1 & 0.2593 & 2.3333 & 1.000 \\
\hline Wind_20 - Turbine_20 & $34.50 / .690$ & 3 & 120 & 220 & 1 & 0.2593 & 2.3333 & 1.000 \\
\hline
\end{tabular}


Table B5.1

Bus Description and Generator Characteristics Plan A 5,000 - 25 kV

WindPACT Turbine Design Scaling Studies

\begin{tabular}{|c|c|c|}
\hline \\
\hline \multicolumn{3}{|c|}{$\begin{array}{r}\text { Collector System Voltage }= \\
\text { Base }\end{array}$} \\
\hline Bus No. & Name & kV \\
\hline & Mission_115 & 115.00 \\
\hline & Mission_25 & 24.9 \\
\hline & Riser_A & 24.9 \\
\hline & Riser_B & 24.9 \\
\hline 10 & Wind_01 & 24.9 \\
\hline 102 & Wind_02 & 24.9 \\
\hline 103 & Wind_03 & 24.9 \\
\hline 10 & Wind_04 & 24.9 \\
\hline 105 & Wind_05 & 24.9 \\
\hline 106 & Wind_06 & 24.9 \\
\hline 107 & Wind_07 & 24.9 \\
\hline $10 \varepsilon$ & Wind_08 & 24.9 \\
\hline $10 s$ & Wind_09 & 24.9 \\
\hline 110 & Wind_10 & 24.9 \\
\hline
\end{tabular}

Power Flow Model

24.9

Pgen

Qmax Qmin

MVAR

Vhold
1.0000

BType

BT

Reference

Load

Load

Load

Load

Load

Load

Load

Load

Load

Load

Load

Load

Load
Bus Type
SC Model

$0.0100 \quad 100.0000$ 
Table B5.1

Bus Description and Generator Characteristics

Plan A 5,000 - 25 kV

WindPACT Turbine Design Scaling Studies

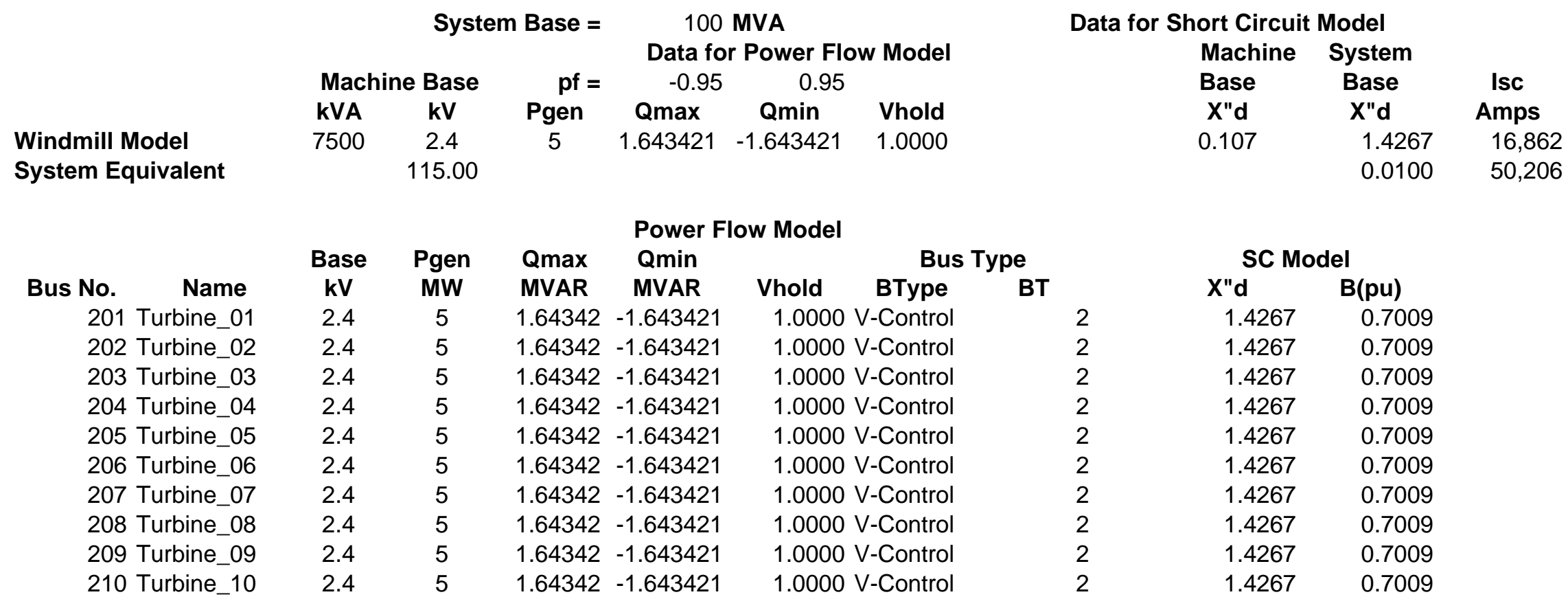


Table B5.2

Distribution Cable and Overhead Line Characteristics

Plan A 5,000 - 25 kV

WindPACT Turbine Design Scaling Studies

\begin{tabular}{|c|c|c|c|c|c|c|c|c|c|c|c|c|c|c|}
\hline \multirow{2}{*}{\multicolumn{2}{|c|}{$\begin{array}{c}\text { Construction } \\
\text { Type }\end{array}$}} & \multirow{2}{*}{$\begin{array}{c}\text { Conductor Size } \\
\text { AWG or kcmil }\end{array}$} & \multirow{2}{*}{$\begin{array}{c}\text { Rated } \\
\text { kV }\end{array}$} & ohms/1 & $00 \mathrm{ft}$. & \multicolumn{2}{|c|}{$\begin{array}{r}\text { Base MVA= } \\
\text { Volt Base= } \\
\text { Z Base= }\end{array}$} & \multicolumn{2}{|c|}{$\begin{array}{c}100 \\
24.90 \\
6.200 \\
\text { PU/1000 ft. }\end{array}$} & \multirow{2}{*}{$\begin{array}{l}\text { Rating } \\
\text { Amps }\end{array}$} & \multicolumn{2}{|c|}{ ohms/1000 ft. } & \multicolumn{2}{|c|}{ PU/1000 ft. } \\
\hline & & & & $\mathbf{R}$ & $\mathbf{X}$ & $\mathrm{pf} / \mathrm{ft}$ & \multirow{2}{*}{$\begin{array}{c}\mathbf{R} \\
0.0342\end{array}$} & \multirow{2}{*}{$\frac{\mathbf{X}}{0.0082}$} & \multirow{2}{*}{$\begin{array}{c}\text { B } \\
0.000122\end{array}$} & & R0 & $\mathrm{XO}$ & Ro & $\mathrm{XO}$ \\
\hline 1 & UG-1/0-25 & $1 / 0 \mathrm{Al}$ & 0.00 & 0.2120 & 0.0510 & 52 & & & & 212 & 0.549 & 0.263 & 0.089 & 0.042 \\
\hline 2 & UG-4/0-25 & 4/0 Al & 0.00 & 0.1070 & 0.0460 & 64 & 0.0173 & 0.0074 & 0.000150 & 312 & 0.334 & 0.11 & 0.054 & 0.018 \\
\hline 3 & UG-500-25 & $500 \mathrm{Al}$ & 0.00 & 0.0480 & 0.0400 & 86 & 0.0077 & 0.0065 & 0.000201 & 490 & 0.158 & 0.04 & 0.025 & 0.006 \\
\hline 4 & OH-SC-715 & 715 ACSR & & 0.0268 & 0.1146 & 3.55 & 0.0043 & 0.0185 & 0.000008 & 849 & 0.082 & 0.556 & 0.013 & 0.090 \\
\hline 5 & $\mathrm{OH}-\mathrm{SC}-477$ & 477 ACSR & & 0.0406 & 0.1214 & 3.37 & 0.0065 & 0.0196 & 0.000008 & 646 & 0.096 & 0.563 & 0.015 & 0.091 \\
\hline 6 & $\mathrm{OH}-\mathrm{SC}-4 / 0$ & 4/0 ACSR & & 0.1085 & 0.1426 & 3.14 & 0.0175 & 0.0230 & 0.000007 & 360 & 0.165 & 0.585 & 0.027 & 0.094 \\
\hline 7 & $\mathrm{OH}-\mathrm{SC}-2 / 0$ & 2/0 ACSR & & 0.1597 & 0.1496 & 3.01 & 0.0258 & 0.0241 & 0.000007 & 276 & 0.217 & 0.592 & 0.035 & 0.095 \\
\hline
\end{tabular}

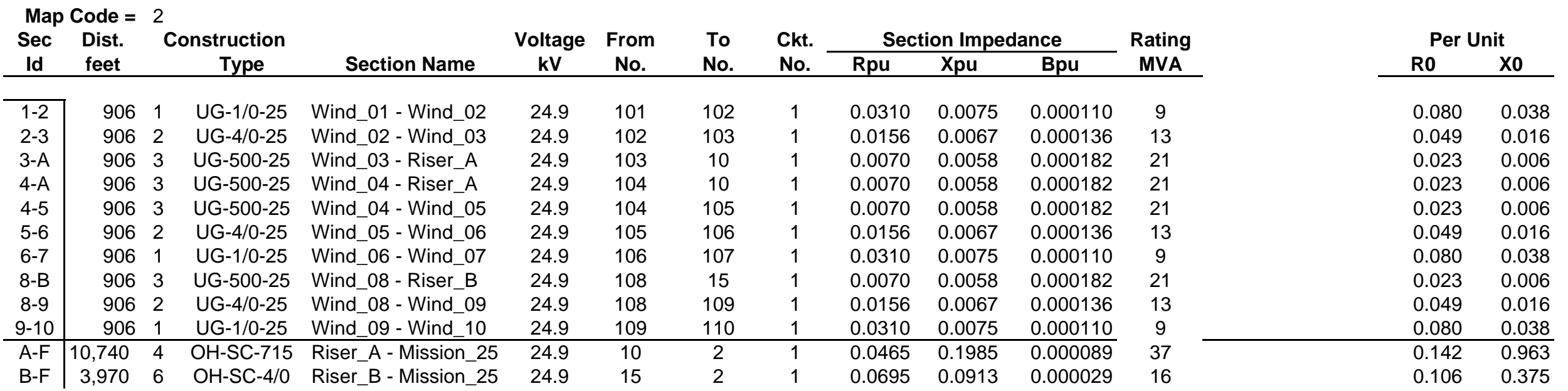


Table B5.3

Transformer Characteristics

Plan A 5,000 - 25 kV

WindPACT Turbine Design Scaling Studies

Transformer Characteristics

Transf. Model Data

\begin{tabular}{|c|c|c|c|c|c|c|c|}
\hline \multirow[b]{4}{*}{ Transformer } & \multicolumn{3}{|c|}{ Transformer Characteristics } & \multicolumn{4}{|c|}{ Transf. Model Data } \\
\hline & & & & & stem & se MVA & 100 \\
\hline & & Base & & & & Imped. ( & st. Base) \\
\hline & Voltage kV & MVA & Rating & $Z \%$ & $\mathbf{X} / \mathbf{R}$ & $R(p u)$ & $X(p u)$ \\
\hline Station Transformer & $115 / 24.9$ & 30 & 50 & $8.5 \%$ & 19 & 0.0149 & 0.2833 \\
\hline Wind Mill Transformer & $24.90 / 2.40$ & 7.5 & 7.5 & $5.75 \%$ & 9 & 0.0852 & 0.7667 \\
\hline
\end{tabular}

\begin{tabular}{|c|c|c|c|c|c|c|c|c|}
\hline \multirow[b]{2}{*}{ Transformer Name } & \multirow[b]{2}{*}{ Voltage kV } & \multirow{2}{*}{$\begin{array}{c}\text { Rating } \\
\text { MVA }\end{array}$} & \multirow{2}{*}{$\begin{array}{l}\text { Tap } \\
\text { No. }\end{array}$} & \multirow{2}{*}{$\begin{array}{l}\text { To } \\
\text { No. }\end{array}$} & \multirow{2}{*}{$\begin{array}{l}\text { Ckt. } \\
\text { Id. }\end{array}$} & \multicolumn{2}{|c|}{ Imped. (Syst. Base) } & \multirow[b]{2}{*}{ Tap } \\
\hline & & & & & & $\mathrm{R}(\mathrm{pu})$ & $\mathrm{X}(\mathrm{pu})$ & \\
\hline Mission_115 - Mission_25 & $115 / 24.9$ & 50 & 1 & 2 & 1 & 0.0149 & 0.2833 & 1.000 \\
\hline Wind_01 - Turbine_01 & $24.90 / 2.40$ & 7.5 & 101 & 201 & 1 & 0.0852 & 0.7667 & 1.000 \\
\hline Wind_02 - Turbine_02 & $24.90 / 2.40$ & 7.5 & 102 & 202 & 1 & 0.0852 & 0.7667 & 1.000 \\
\hline Wind_03 - Turbine_03 & $24.90 / 2.40$ & 7.5 & 103 & 203 & 1 & 0.0852 & 0.7667 & 1.000 \\
\hline Wind_04 - Turbine_04 & $24.90 / 2.40$ & 7.5 & 104 & 204 & 1 & 0.0852 & 0.7667 & 1.000 \\
\hline Wind_05 - Turbine_05 & $24.90 / 2.40$ & 7.5 & 105 & 205 & 1 & 0.0852 & 0.7667 & 1.000 \\
\hline Wind_06 - Turbine_06 & $24.90 / 2.40$ & 7.5 & 106 & 206 & 1 & 0.0852 & 0.7667 & 1.000 \\
\hline Wind_07 - Turbine_07 & $24.90 / 2.40$ & 7.5 & 107 & 207 & 1 & 0.0852 & 0.7667 & 1.000 \\
\hline Wind_08 - Turbine_08 & $24.90 / 2.40$ & 7.5 & 108 & 208 & 1 & 0.0852 & 0.7667 & 1.000 \\
\hline Wind_09 - Turbine_09 & $24.90 / 2.40$ & 7.5 & 109 & 209 & 1 & 0.0852 & 0.7667 & 1.000 \\
\hline Wind_10 - Turbine_10 & $24.90 / 2.40$ & 7.5 & 110 & 210 & 1 & 0.0852 & 0.7667 & 1.000 \\
\hline
\end{tabular}


Table B6.1

Bus Description and Generator Characteristics Plan A 5,000 - 35 kV

WindPACT Turbine Design Scaling Studies

\begin{tabular}{|c|c|c|}
\hline \multicolumn{3}{|c|}{ Collector System Voltage $=$} \\
\hline & & Base \\
\hline Bus No. & Name & kV \\
\hline & ission_115 & 115.00 \\
\hline & ission 35 & 34.5 \\
\hline & ser $A$ & 34.5 \\
\hline & ser $B$ & 34.5 \\
\hline 10 & ind 01 & 34.5 \\
\hline 10 & ind 02 & 34.5 \\
\hline 103 & ind 03 & 34.5 \\
\hline 10 & ind_04 & 34.5 \\
\hline 105 & ind_05 & 34.5 \\
\hline 106 & lind_06 & 34.5 \\
\hline 107 & lind_07 & 34.5 \\
\hline $10 \varepsilon$ & lind 08 & 34.5 \\
\hline $10 \varsigma$ & lind 09 & 34.5 \\
\hline 110 & lind 10 & 34.5 \\
\hline
\end{tabular}

Power Flow Model

34.5

Pgen

MW

$\begin{array}{cc}\text { Qmax } & \text { Qmin } \\ \text { MVAR } & \text { MVAR }\end{array}$

Vhold
1.0000

Bus Type

BType

BT

Reference

Load

Load

Load

Load

Load

Load

Load

Load

Load

Load

Load

Load

Load
SC Model

$\mathrm{B}(\mathrm{pu})$

$0.0100 \quad 100.0000$ 
Table B6.1

Bus Description and Generator Characteristics

Plan A 5,000 - 35 kV

WindPACT Turbine Design Scaling Studies

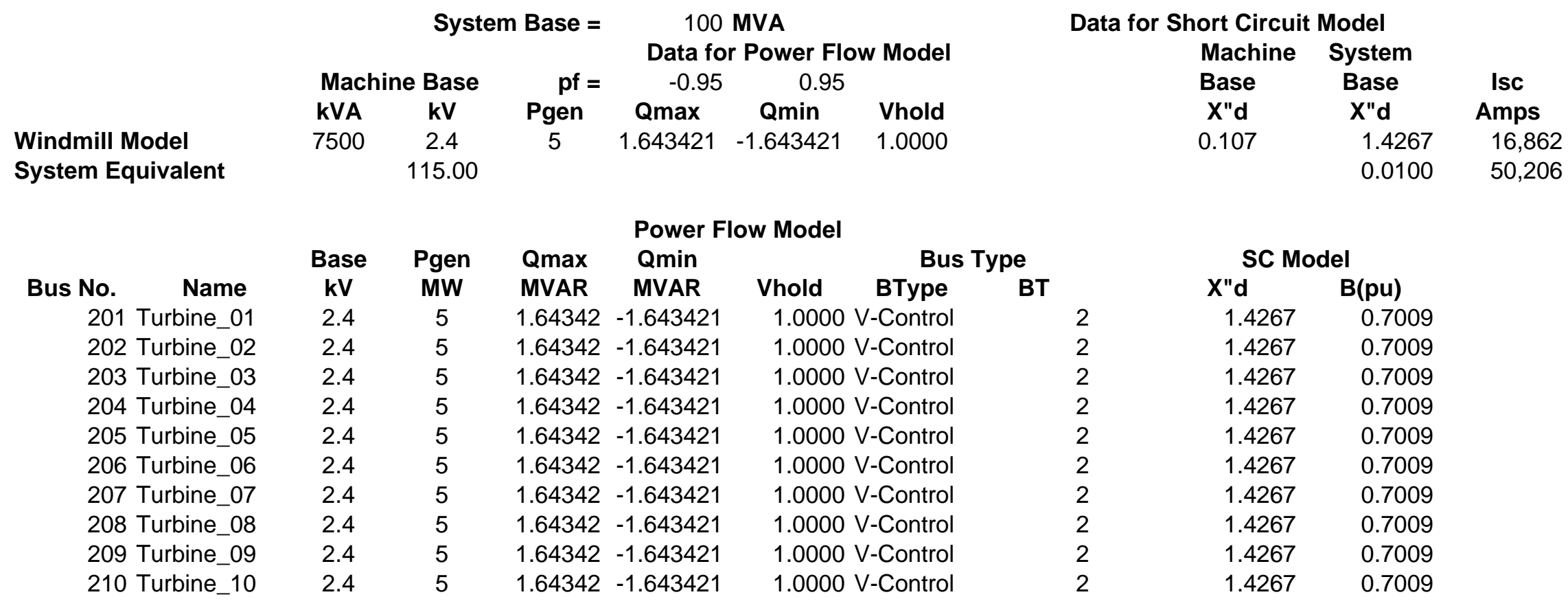


Table B6.2

Distribution Cable and Overhead Line Characteristics

Plan A 5,000 - 35 kV

WindPACT Turbine Design Scaling Studies

\begin{tabular}{|c|c|c|c|c|c|c|c|c|c|c|c|c|c|c|}
\hline \multirow{2}{*}{\multicolumn{2}{|c|}{$\begin{array}{c}\text { Construction } \\
\text { Type }\end{array}$}} & \multirow{2}{*}{$\begin{array}{c}\text { Conductor Size } \\
\text { AWG or kcmil }\end{array}$} & \multirow{2}{*}{$\begin{array}{c}\text { Rated } \\
\text { kV }\end{array}$} & ohms/1 & $00 \mathrm{ft}$. & \multicolumn{2}{|c|}{$\begin{array}{r}\text { Base MVA= } \\
\text { Volt Base= } \\
\text { Z Base= }\end{array}$} & \multicolumn{2}{|c|}{$\begin{array}{c}100 \\
34.50 \\
11.903 \\
\text { PU/1000 ft. }\end{array}$} & \multirow{2}{*}{$\begin{array}{l}\text { Rating } \\
\text { Amps }\end{array}$} & \multicolumn{2}{|c|}{ ohms/1000 ft. } & \multicolumn{2}{|c|}{ PU/1000 ft. } \\
\hline & & & & $\mathbf{R}$ & $\mathbf{X}$ & $\mathrm{pf} / \mathrm{ft}$ & \multirow{2}{*}{$\begin{array}{c}\mathbf{R} \\
0.0178\end{array}$} & \multirow{2}{*}{$\frac{X}{0.0046}$} & \multirow{2}{*}{$\begin{array}{c}\text { B } \\
0.000233\end{array}$} & & R0 & $\mathrm{XO}$ & R0 & $\mathrm{XO}$ \\
\hline 1 & UG-1/0-25 & $1 / 0 \mathrm{Al}$ & 34.50 & 0.2120 & 0.0550 & 52 & & & & 212 & 0.545 & 0.267 & 0.046 & 0.022 \\
\hline 2 & UG-4/0-25 & $4 / 0 \mathrm{Al}$ & 34.50 & 0.1070 & 0.0490 & 64 & 0.0090 & 0.0041 & 0.000287 & 312 & 0.333 & 0.113 & 0.028 & 0.009 \\
\hline 3 & UG-500-25 & $500 \mathrm{Al}$ & 34.50 & 0.0480 & 0.0400 & 86 & 0.0040 & 0.0034 & 0.000386 & 490 & 0.158 & 0.044 & 0.013 & 0.004 \\
\hline 4 & $\mathrm{OH}-\mathrm{SC}-715$ & 715 ACSR & & 0.0268 & 0.1146 & 3.55 & 0.0023 & 0.0096 & 0.000016 & 849 & 0.082 & 0.556 & 0.007 & 0.047 \\
\hline 5 & $\mathrm{OH}-\mathrm{SC}-477$ & 477 ACSR & & 0.0406 & 0.1214 & 3.37 & 0.0034 & 0.0102 & 0.000015 & 646 & 0.096 & 0.563 & 0.008 & 0.047 \\
\hline 6 & $\mathrm{OH}-\mathrm{SC}-2 / 0$ & 2/0 ACSR & & 0.1597 & 0.1496 & 3.01 & 0.0134 & 0.0126 & 0.000014 & 276 & 0.217 & 0.592 & 0.018 & 0.050 \\
\hline
\end{tabular}

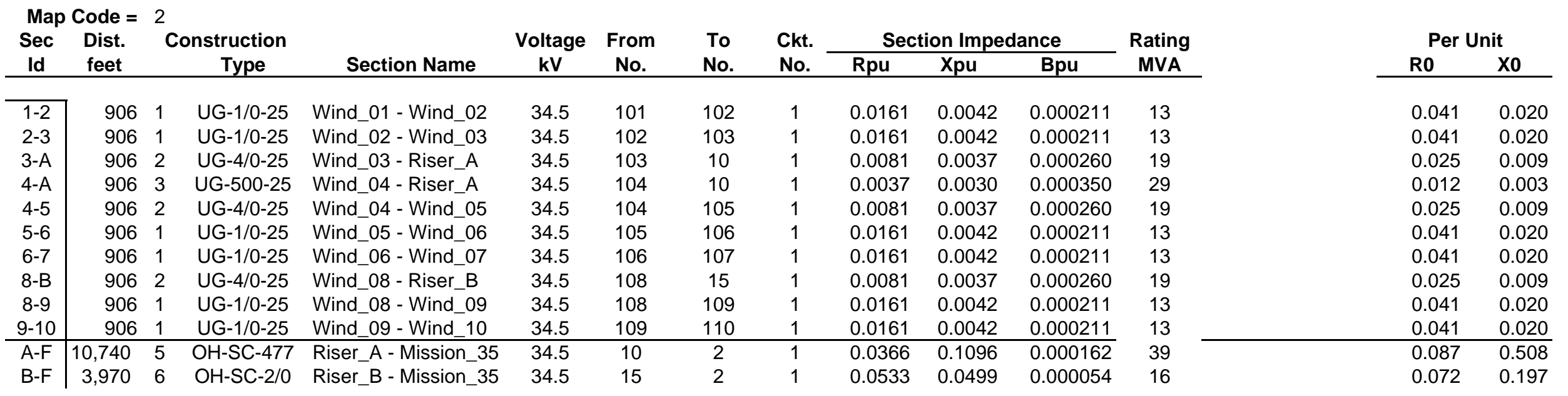


Table B6.3

Transformer Characteristics

Plan A 5,000 - 35 kV

WindPACT Turbine Design Scaling Studies

\begin{tabular}{|c|c|c|c|c|c|c|c|}
\hline \multirow[b]{4}{*}{ Transformer } & \multicolumn{3}{|c|}{ Transformer Characteristics } & \multicolumn{4}{|c|}{ Transf. Model Data } \\
\hline & \multirow[b]{3}{*}{ Voltage kV } & \multirow{3}{*}{$\begin{array}{l}\text { Base } \\
\text { MVA }\end{array}$} & \multirow{3}{*}{$\begin{array}{l}\text { Top } \\
\text { Rating }\end{array}$} & \multirow{2}{*}{\multicolumn{4}{|c|}{$\begin{array}{c}\text { System Base MVA }= \\
\text { Imped. (Syst. Base) }\end{array}$}} \\
\hline & & & & & & & \\
\hline & & & & $Z \%$ & $\mathbf{X} / \mathbf{R}$ & $\mathbf{R}(\mathrm{pu})$ & $X(p u)$ \\
\hline Station Transformer & $115 / 34.5$ & 30 & 50 & $8.5 \%$ & 19 & 0.0149 & 0.2833 \\
\hline Wind Mill Transformer & $34.50 / 2.40$ & 7.5 & 7.5 & $5.75 \%$ & 9 & 0.0852 & 0.7667 \\
\hline
\end{tabular}

\begin{tabular}{|c|c|c|c|c|c|c|c|c|}
\hline \multirow[b]{2}{*}{ Transformer Name } & \multirow[b]{2}{*}{ Voltage kV } & \multirow{2}{*}{$\begin{array}{c}\text { Rating } \\
\text { MVA }\end{array}$} & \multirow{2}{*}{$\begin{array}{l}\text { Tap } \\
\text { No. }\end{array}$} & \multirow{2}{*}{$\begin{array}{l}\text { To } \\
\text { No. }\end{array}$} & \multirow{2}{*}{$\begin{array}{l}\text { Ckt. } \\
\text { Id. }\end{array}$} & \multicolumn{2}{|c|}{ Imped. (Syst. Base) } & \multirow[b]{2}{*}{ Tap } \\
\hline & & & & & & $\mathrm{R}(\mathrm{pu})$ & $X(p u)$ & \\
\hline Mission_115 - Mission_35 & $115 / 34.5$ & 50 & 1 & 2 & 1 & 0.0149 & 0.2833 & 1.000 \\
\hline Wind_01 - Turbine_01 & $34.50 / 2.40$ & 7.5 & 101 & 201 & 1 & 0.0852 & 0.7667 & 1.000 \\
\hline Wind_02 - Turbine_02 & $34.50 / 2.40$ & 7.5 & 102 & 202 & 1 & 0.0852 & 0.7667 & 1.000 \\
\hline Wind_03 - Turbine_03 & $34.50 / 2.40$ & 7.5 & 103 & 203 & 1 & 0.0852 & 0.7667 & 1.000 \\
\hline Wind_04 - Turbine_04 & $34.50 / 2.40$ & 7.5 & 104 & 204 & 1 & 0.0852 & 0.7667 & 1.000 \\
\hline Wind_05 - Turbine_05 & $34.50 / 2.40$ & 7.5 & 105 & 205 & 1 & 0.0852 & 0.7667 & 1.000 \\
\hline Wind_06 - Turbine_06 & $34.50 / 2.40$ & 7.5 & 106 & 206 & 1 & 0.0852 & 0.7667 & 1.000 \\
\hline Wind_07 - Turbine_07 & $34.50 / 2.40$ & 7.5 & 107 & 207 & 1 & 0.0852 & 0.7667 & 1.000 \\
\hline Wind_08 - Turbine_08 & $34.50 / 2.40$ & 7.5 & 108 & 208 & 1 & 0.0852 & 0.7667 & 1.000 \\
\hline Wind_09 - Turbine_09 & $34.50 / 2.40$ & 7.5 & 109 & 209 & 1 & 0.0852 & 0.7667 & 1.000 \\
\hline Wind_10 - Turbine_10 & $34.50 / 2.40$ & 7.5 & 110 & 210 & 1 & 0.0852 & 0.7667 & 1.000 \\
\hline
\end{tabular}


Table B7.1

Plan A 10,000 - 25 kV

Bus Description and Generator Characteristics

WindPACT Turbine Design Scaling Studies

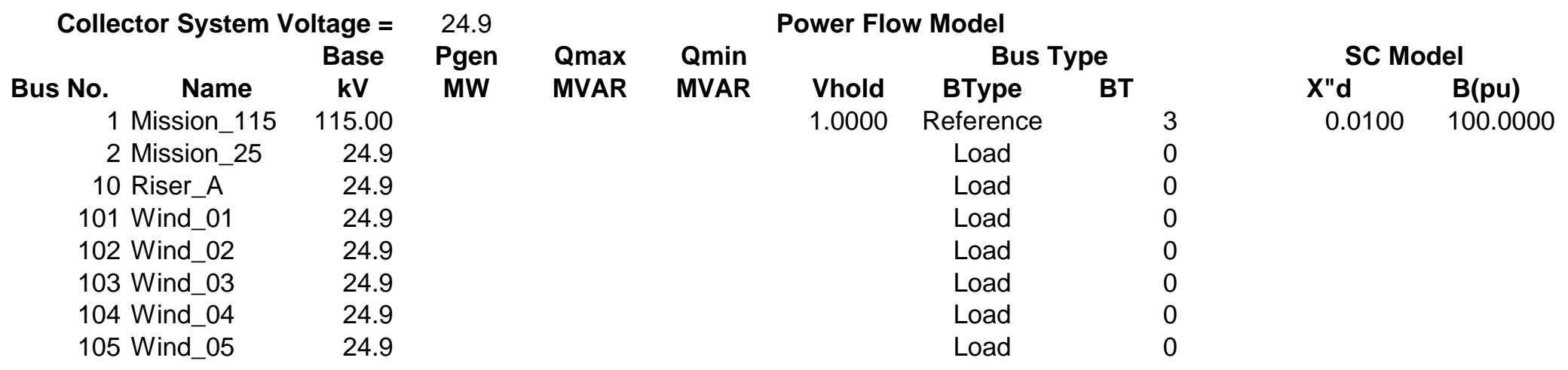

\begin{tabular}{|c|c|c|c|c|c|c|c|c|c|c|c|}
\hline & & Syst & Base = & $\begin{array}{r}100 \\
\text { Data fo }\end{array}$ & MVA & Andol & & Dat & ort Circuit & Model & \\
\hline & Mach & e Base & pf $=$ & -0.95 & 0.95 & & & & Base & Base & Isc \\
\hline & kVA & kV & Pgen & Qmax & Qmin & Vhold & & & $X " d$ & X"d & Amps \\
\hline Windmill Model & 11111 & 4.16 & 10 & 3.286841 & -3.286841 & 1.0000 & & & 0.107 & 0.9630 & 14,412 \\
\hline System Equivalent & & 115.00 & & & & & & & & 0.0100 & 50,206 \\
\hline & Base & Pgen & Qmax & $\begin{array}{l}\text { Power FI } \\
\text { Qmin }\end{array}$ & ow Model & Bus & & & SC Mc & & \\
\hline Bus No. & kV & MW & MVAR & MVAR & Vhold & BType & BT & & $X " d$ & $B(p u)$ & \\
\hline 201 Turbine_01 & 4.16 & 10 & 3.28684 & -3.286841 & 1.0000 & V-Control & & 2 & 0.9630 & 1.0384 & \\
\hline 202 Turbine_02 & 4.16 & 10 & 3.28684 & -3.286841 & 1.0000 & V-Control & & 2 & 0.9630 & 1.0384 & \\
\hline 203 Turbine_03 & 4.16 & 10 & 3.28684 & -3.286841 & 1.0000 & V-Control & & 2 & 0.9630 & 1.0384 & \\
\hline 204 Turbine_04 & 4.16 & 10 & 3.28684 & -3.286841 & 1.0000 & V-Control & & 2 & 0.9630 & 1.0384 & \\
\hline 205 Turbine_05 & 4.16 & 10 & 3.28684 & -3.286841 & 1.0000 & V-Control & & 2 & 0.9630 & 1.0384 & \\
\hline
\end{tabular}


Table B7.2

Distribution Cable and Overhead Line Characteristics

Plan A 10,000 - 25 kV

WindPACT Turbine Design Scaling Studies

\begin{tabular}{|c|c|c|c|c|c|c|c|c|c|c|c|c|c|c|}
\hline \multirow{2}{*}{\multicolumn{2}{|c|}{$\begin{array}{c}\text { Construction } \\
\text { Type }\end{array}$}} & \multirow{2}{*}{$\begin{array}{c}\text { Conductor Size } \\
\text { AWG or kcmil }\end{array}$} & \multirow{2}{*}{$\begin{array}{c}\text { Rated } \\
\text { kV }\end{array}$} & ohms/ & $00 \mathrm{ft}$. & \multicolumn{2}{|c|}{$\begin{array}{r}\text { Base MVA= } \\
\text { Volt Base= } \\
\text { Z Base= }\end{array}$} & \multicolumn{2}{|c|}{$\begin{array}{r}100 \\
24.90 \\
6.200 \\
\mathbf{U} / 1000\end{array}$} & \multirow{2}{*}{$\begin{array}{l}\text { Rating } \\
\text { Amps }\end{array}$} & \multicolumn{2}{|c|}{ ohms/1000 ft. } & \multicolumn{2}{|c|}{ PU/1000 ft. } \\
\hline & & & & $\mathbf{R}$ & $\mathbf{X}$ & $\mathrm{pf} / \mathrm{ft}$ & \multirow{2}{*}{$\begin{array}{c}\mathbf{R} \\
0.0342\end{array}$} & \multirow{2}{*}{$\begin{array}{c}\mathbf{X} \\
0.0082\end{array}$} & \multirow{2}{*}{$\begin{array}{c}\text { B } \\
0.000122\end{array}$} & & Ro & X0 & Ro & $\mathrm{XO}$ \\
\hline 1 & UG-1/0-25 & $1 / 0 \mathrm{Al}$ & 24.90 & 0.2120 & 0.0510 & 52 & & & & 212 & 0.549 & 0.263 & 0.089 & 0.042 \\
\hline 2 & UG-4/0-25 & $4 / 0 \mathrm{Al}$ & 24.90 & 0.1070 & 0.0460 & 64 & 0.0173 & 0.0074 & 0.000150 & 312 & 0.334 & 0.11 & 0.054 & 0.018 \\
\hline 3 & UG-500-25 & $500 \mathrm{Al}$ & 24.90 & 0.0480 & 0.0400 & 86 & 0.0077 & 0.0065 & 0.000201 & 490 & 0.158 & 0.04 & 0.025 & 0.006 \\
\hline 4 & OH-SC-795 & 795 ACSR & & 0.0242 & 0.1135 & 3.59 & 0.0039 & 0.0183 & 0.000008 & 907 & 0.126 & 0.529 & 0.020 & 0.085 \\
\hline 5 & OH-DC1-795 & 795 ACSR & & 0.0242 & 0.1056 & 3.86 & 0.0039 & 0.0170 & 0.000009 & 907 & 0.126 & 0.492 & 0.020 & 0.079 \\
\hline 6 & OH-DC2-795 & 796 ACSR & & 0.0242 & 0.1056 & 3.86 & 0.0039 & 0.0170 & 0.000009 & 907 & 0.126 & 0.492 & 0.020 & 0.079 \\
\hline
\end{tabular}

Map Code $=2$

\begin{tabular}{|c|c|c|c|c|c|c|c|c|c|c|c|c|c|c|}
\hline \multirow{2}{*}{$\begin{array}{l}\text { Sec } \\
\text { Id }\end{array}$} & \multirow{2}{*}{$\begin{array}{l}\text { Dist. } \\
\text { feet }\end{array}$} & \multirow{2}{*}{\multicolumn{2}{|c|}{$\begin{array}{c}\text { Construction } \\
\text { Type }\end{array}$}} & \multirow[b]{2}{*}{ Section Name } & \multirow{2}{*}{$\begin{array}{c}\text { Voltage } \\
\text { kV }\end{array}$} & \multirow{2}{*}{$\begin{array}{c}\text { From } \\
\text { No. }\end{array}$} & \multirow{2}{*}{$\begin{array}{r}\text { To } \\
\text { No. } \\
\end{array}$} & \multirow{2}{*}{$\begin{array}{l}\text { Ckt. } \\
\text { No. }\end{array}$} & \multicolumn{3}{|c|}{ Section Impedance } & \multirow{2}{*}{$\begin{array}{c}\text { Rating } \\
\text { MVA }\end{array}$} & \multicolumn{2}{|c|}{ Per Unit } \\
\hline & & & & & & & & & Rpu & $\mathrm{Xpu}$ & $\mathrm{Bpu}$ & & Ro & XO \\
\hline $1-2$ & 1,283 & 2 & UG-4/0-25 & Wind_01 - Wind_02 & 24.9 & 101 & 102 & 1 & 0.0221 & 0.0095 & 0.000156 & 13 & 0.069 & 0.023 \\
\hline $2-A$ & 1,283 & 3 & UG-500-25 & Wind_02 - Riser_A & 24.9 & 102 & 10 & 1 & 0.0099 & 0.0083 & 0.000156 & 21 & 0.033 & 0.008 \\
\hline 3-A & 800 & 2 & UG-4/0-25 & Wind_03 - Riser_A & 24.9 & 103 & 10 & 1 & 0.0138 & 0.0059 & 0.000097 & 13 & 0.043 & 0.014 \\
\hline $4-A$ & 1,500 & 3 & UG-500-25 & Wind_04 - Riser_A & 24.9 & 104 & 10 & 1 & 0.0116 & 0.0097 & 0.000182 & 21 & 0.038 & 0.010 \\
\hline $4-5$ & 1,283 & 2 & UG-4/0-25 & Wind_04 - Wind_05 & 24.9 & 104 & 105 & 1 & 0.0221 & 0.0095 & 0.000156 & 13 & 0.069 & 0.023 \\
\hline$A-F$ & 4,500 & 4 & $\mathrm{OH}-\mathrm{SC}-795$ & Riser_A - Mission_25 & 24.9 & 10 & 2 & 1 & 0.0176 & 0.0824 & 0.000038 & 39 & 0.091 & 0.384 \\
\hline
\end{tabular}


Table B7.3

Transformer Characteristics

Plan A 10,000 - 25

WindPACT Turbine Scaling Studies

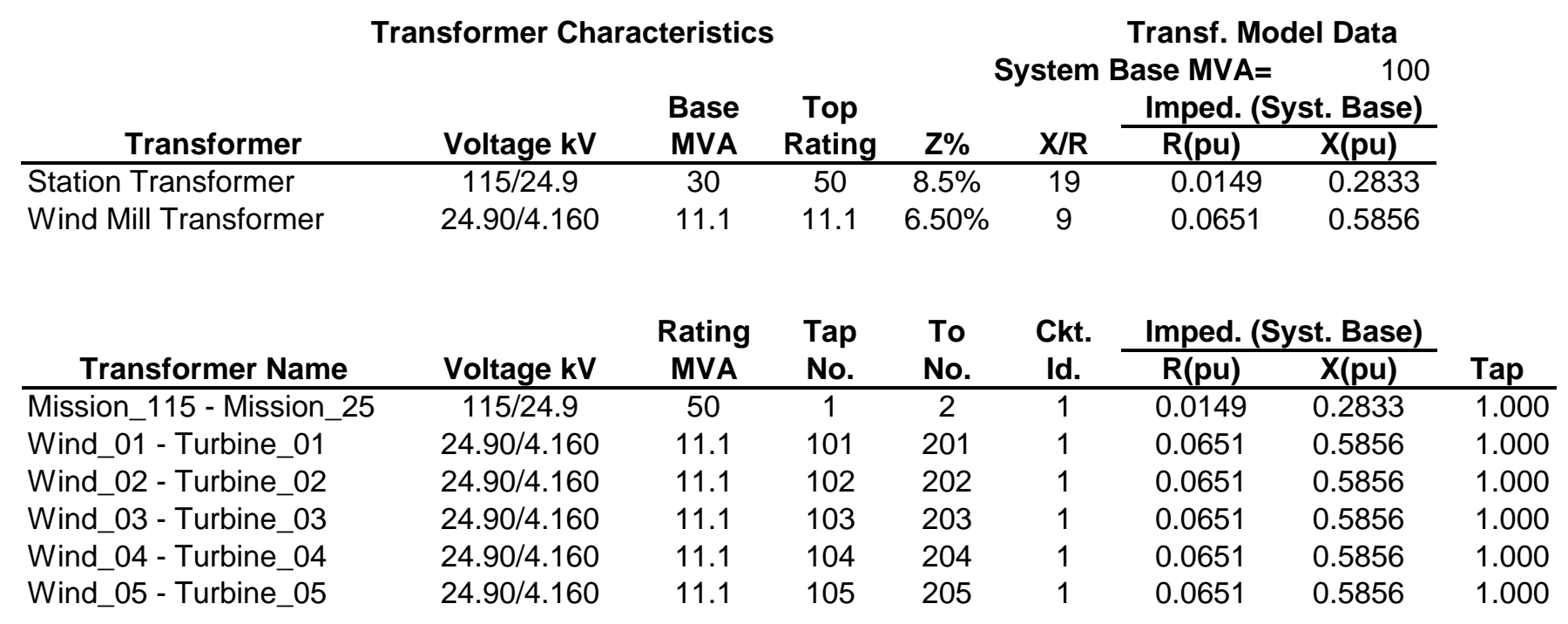


Table B8.1

Bus Description and Generator Characteristics

Plan B 10,000 - 35 kV

WindPACT Turbine Design Scaling Studies

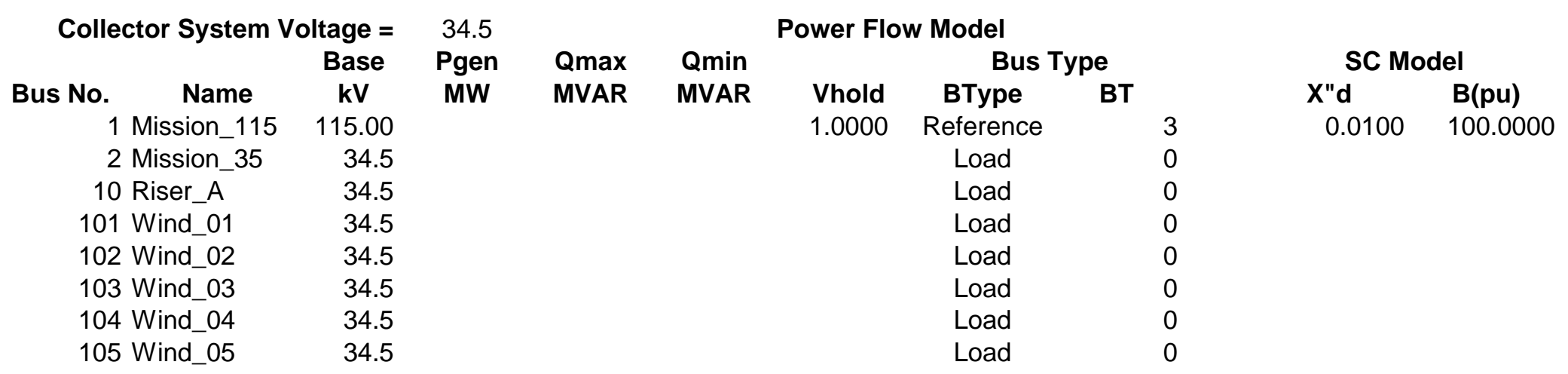

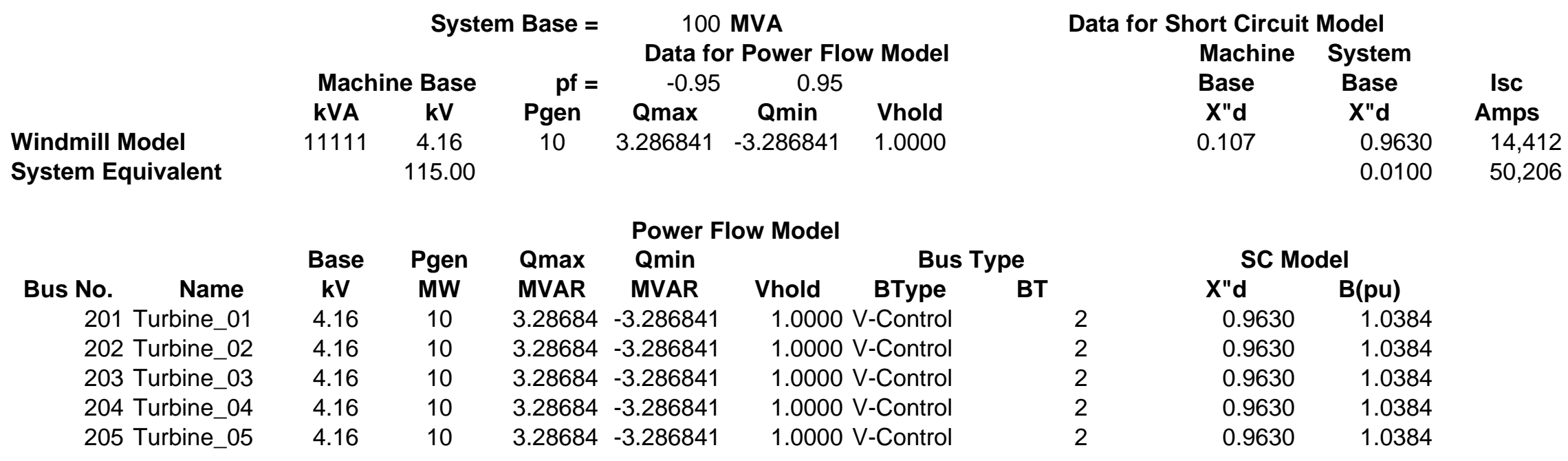


Table B8.2

Distribution Cable and Overhead Line Characteristics

Plan B 10,000 - 35 kV

WindPACT Turbine Design Scaling Studies

\begin{tabular}{|c|c|c|c|c|c|c|c|c|c|c|c|c|c|c|}
\hline \multirow{2}{*}{\multicolumn{2}{|c|}{$\begin{array}{c}\text { Construction } \\
\text { Type }\end{array}$}} & \multirow{2}{*}{$\begin{array}{c}\text { Conductor Size } \\
\text { AWG or kcmil }\end{array}$} & \multirow{2}{*}{$\begin{array}{c}\text { Rated } \\
\text { kV }\end{array}$} & ohms/1 & $00 \mathrm{ft}$. & \multicolumn{2}{|c|}{$\begin{array}{r}\text { Base MVA= } \\
\text { Volt Base= } \\
\text { Z Base= }\end{array}$} & \multicolumn{2}{|c|}{$\begin{array}{c}100 \\
34.50 \\
11.903 \\
\text { PU/1000 ft. }\end{array}$} & \multirow{2}{*}{$\begin{array}{l}\text { Rating } \\
\text { Amps }\end{array}$} & \multicolumn{2}{|c|}{ ohms/1000 ft. } & \multicolumn{2}{|c|}{ PU/1000 ft. } \\
\hline & & & & $\mathbf{R}$ & $\mathbf{X}$ & $\mathrm{pf} / \mathrm{ft}$ & \multirow{2}{*}{$\begin{array}{c}\mathbf{R} \\
0.0178\end{array}$} & \multirow{2}{*}{$\frac{X}{0.0046}$} & \multirow{2}{*}{$\begin{array}{c}\text { B } \\
0.000166\end{array}$} & & R0 & $\mathrm{XO}$ & R0 & $\mathrm{XO}$ \\
\hline 1 & UG-1/0-35 & $1 / 0 \mathrm{Al}$ & 34.50 & 0.2120 & 0.0550 & 37 & & & & 212 & 0.545 & 0.267 & 0.046 & 0.022 \\
\hline 2 & UG-4/0-35 & $4 / 0 \mathrm{Al}$ & 34.50 & 0.1070 & 0.0490 & 45 & 0.0090 & 0.0041 & 0.000202 & 312 & 0.333 & 0.113 & 0.028 & 0.009 \\
\hline 3 & UG-500-35 & $500 \mathrm{Al}$ & 34.50 & 0.0470 & 0.0420 & 60 & 0.0039 & 0.0035 & 0.000269 & 490 & 0.158 & 0.044 & 0.013 & 0.004 \\
\hline 4 & OH-SC-795 & 795 ACSR & & 0.0242 & 0.1135 & 3.59 & 0.0020 & 0.0095 & 0.000016 & 907 & 0.126 & 0.529 & 0.011 & 0.044 \\
\hline 5 & $\mathrm{OH}-\mathrm{DC1}-795$ & 795 ACSR & & 0.0242 & 0.1056 & 3.86 & 0.0020 & 0.0089 & 0.000017 & 907 & 0.126 & 0.492 & 0.011 & 0.041 \\
\hline 6 & OH-DC2-795 & 796 ACSR & & 0.0242 & 0.1056 & 3.86 & 0.0020 & 0.0089 & 0.000017 & 907 & 0.126 & 0.492 & 0.011 & 0.041 \\
\hline
\end{tabular}

Map Code $=2$

\begin{tabular}{|c|c|c|c|c|c|c|c|c|c|c|c|c|c|c|}
\hline \multirow{2}{*}{$\begin{array}{l}\text { Sec } \\
\text { Id }\end{array}$} & \multirow{2}{*}{$\begin{array}{l}\text { Dist. } \\
\text { feet }\end{array}$} & \multirow{2}{*}{\multicolumn{2}{|c|}{$\begin{array}{c}\text { Construction } \\
\text { Type }\end{array}$}} & \multirow[b]{2}{*}{ Section Name } & \multirow{2}{*}{$\begin{array}{c}\text { Voltage } \\
\text { kV }\end{array}$} & \multirow{2}{*}{$\begin{array}{l}\text { From } \\
\text { No. }\end{array}$} & \multirow{2}{*}{$\begin{array}{l}\text { To } \\
\text { No. } \\
\end{array}$} & \multirow{2}{*}{$\begin{array}{l}\text { Ckt. } \\
\text { No. }\end{array}$} & \multicolumn{3}{|c|}{ Section Impedance } & \multirow{2}{*}{$\begin{array}{l}\text { Rating } \\
\text { MVA }\end{array}$} & \multicolumn{2}{|c|}{ Per Unit } \\
\hline & & & & & & & & & Rpu & Xpu & Bpu & & Ro & XO \\
\hline $1-2$ & 1,283 & 1 & UG-1/0-35 & Wind_01 - Wind_02 & 34.5 & 101 & 102 & 1 & 0.0229 & 0.0059 & 0.000213 & 13 & 0.059 & 0.029 \\
\hline $2-A$ & 1,283 & 3 & UG-500-35 & Wind_02 - Riser_A & 34.5 & 102 & 10 & 1 & 0.0051 & 0.0045 & 0.000213 & 29 & 0.017 & 0.005 \\
\hline $3-A$ & 800 & 1 & UG-1/0-35 & Wind_03 - Riser_A & 34.5 & 103 & 10 & 1 & 0.0142 & 0.0037 & 0.000133 & 13 & 0.037 & 0.018 \\
\hline $4-A$ & 1,500 & 3 & UG-500-35 & Wind_04 - Riser_A & 34.5 & 104 & 10 & 1 & 0.0059 & 0.0053 & 0.000249 & 29 & 0.020 & 0.006 \\
\hline $4-5$ & 1,283 & 1 & UG-1/0-35 & Wind_04 - Wind_05 & 34.5 & 104 & 105 & 1 & 0.0229 & 0.0059 & 0.000213 & 13 & 0.059 & 0.029 \\
\hline$A-F$ & 4,500 & 4 & $\mathrm{OH}-\mathrm{SC}-795$ & Riser_A - Mission_35 & 34.5 & 10 & 2 & 1 & 0.0091 & 0.0429 & 0.000072 & 54 & 0.048 & 0.200 \\
\hline
\end{tabular}


Table B8.3

Transformer Characteristics

Plan B 10,000 - 35 kV

WindPACT Turbine Scaling Studies

\begin{tabular}{|c|c|c|c|c|c|c|c|c|}
\hline \multirow{2}{*}{\multicolumn{5}{|c|}{ Transformer Characteristics }} & \multicolumn{4}{|c|}{ Transf. Model Data } \\
\hline & & & & & stem & ase MVA: & 100 & \\
\hline \multirow[b]{2}{*}{ Transformer } & \multirow[b]{2}{*}{ Voltage kV } & \multirow{2}{*}{$\begin{array}{l}\text { Base } \\
\text { MVA }\end{array}$} & \multirow{2}{*}{$\begin{array}{c}\text { Top } \\
\text { Rating }\end{array}$} & \multicolumn{4}{|c|}{ 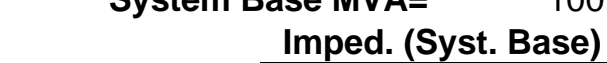 } & \\
\hline & & & & $Z \%$ & $\mathbf{X} / \mathbf{R}$ & $\mathbf{R}(\mathrm{pu})$ & $X(p u)$ & \\
\hline Station Transformer & $115 / 34.5$ & 30 & 50 & $8.5 \%$ & 19 & 0.0149 & 0.2833 & \\
\hline Wind Mill Transformer & $34.50 / 4.160$ & 11.1 & 11.1 & $7.00 \%$ & 9 & 0.0701 & 0.6306 & \\
\hline \multirow[b]{2}{*}{ Transformer Name } & \multirow[b]{2}{*}{ Voltage kV } & \multirow{2}{*}{$\begin{array}{c}\text { Rating } \\
\text { MVA }\end{array}$} & \multirow{2}{*}{$\begin{array}{l}\text { Tap } \\
\text { No. }\end{array}$} & \multirow{2}{*}{$\begin{array}{l}\text { To } \\
\text { No. }\end{array}$} & \multirow{2}{*}{$\begin{array}{l}\text { Ckt. } \\
\text { Id. }\end{array}$} & \multicolumn{2}{|c|}{ Imped. (Syst. Base) } & \\
\hline & & & & & & $R(p u)$ & $\mathrm{X}(\mathrm{pu})$ & Tap \\
\hline Mission_115 - Mission_35 & $115 / 34.5$ & 50 & 1 & 2 & 1 & 0.0149 & 0.2833 & 1.000 \\
\hline Wind_01 - Turbine_01 & $34.50 / 4.160$ & 11.1 & 101 & 201 & 1 & 0.0701 & 0.6306 & 1.000 \\
\hline Wind_02 - Turbine_02 & $34.50 / 4.160$ & 11.1 & 102 & 202 & 1 & 0.0701 & 0.6306 & 1.000 \\
\hline Wind_03 - Turbine_03 & $34.50 / 4.160$ & 11.1 & 103 & 203 & 1 & 0.0701 & 0.6306 & 1.000 \\
\hline Wind_04 - Turbine_04 & $34.50 / 4.160$ & 11.1 & 104 & 204 & 1 & 0.0701 & 0.6306 & 1.000 \\
\hline Wind_05 - Turbine_05 & $34.50 / 4.160$ & 11.1 & 105 & 205 & 1 & 0.0701 & 0.6306 & 1.000 \\
\hline
\end{tabular}




$\begin{array}{ll}\text { Table C1 } & \text { Summary } \\ \text { Table C2 } & \text { Power Flow Results }-750 \mathrm{~kW} / 15 \mathrm{kV} \\ \text { Table C3 } & \text { Power Flow Results }-750 \mathrm{~kW} / 25 \mathrm{kV} \\ \text { Table C4 } & \text { Power Flow Results }-2,500 \mathrm{~kW} / 25 \mathrm{kV} \\ \text { Table C5 } & \text { Power Flow Results }-2,500 \mathrm{~kW} / 35 \mathrm{kV} \\ \text { Table C6 } & \text { Power Flow Results }-5,000 \mathrm{~kW} / 25 \mathrm{kV} \\ \text { Table C7 } & \text { Power Flow Results }-5,000 \mathrm{~kW} / 35 \mathrm{kV} \\ \text { Table C8 } & \text { Power Flow Results }-10,000 \mathrm{~kW} / 25 \mathrm{kV} \\ \text { Table C9 } & \text { Power Flow Results }-10,000 \mathrm{~kW} / 35 \mathrm{kV}\end{array}$




\section{APPENDIX C \\ POWER FLOW STUDY RESULTS}

The power flow analysis provides the following calculations:

1. Computes the power flow on each cable, overhead line, and transformer and compares it with the ratings of these facilities.

2. Computes the voltage drop across the system.

3. Computes the watt and VAR losses.

To evaluate ratings and voltage drop, the power flow analysis is typically made for peak generating conditions.

The power flow analysis in this report evaluated only the collector system. Therefore, the utility $115-\mathrm{kV}$ connection was assumed to be an infinite bus with constant $100 \%$ voltage. A separate power flow study would be required to evaluate the impact of the wind farm on the utility 115$\mathrm{kV}$ transmission system. CAI made a quick review of the $115-\mathrm{kV}$ line serving Mission and also reviewed a report prepared by WAPA. The $115-\mathrm{kV}$ line is capable of accepting the 50-MW generation.

Based on an infinite bus at the utility connection, the voltage drop across the proposed 13.8-kV collector system for the $750-\mathrm{kW}$ plan is only $1.6 \%$ at peak generation. This system demonstrates good voltage regulation and indicates that other voltage regulating equipment, such as load-tapchanging (LTC) transformers or voltage regulators, is not required. For the remaining plans, the voltage drop across the $34.5-$ and $24.9-\mathrm{kV}$ systems is less than $1.5 \%$. The addition of generation (MW and MVAR) to the utility $115-\mathrm{kV}$ system will impact voltage regulation on the utility $115-$ $\mathrm{kV}$ bus. The proposed system includes capacitive compensation to provide the generation to the utility at unity power factor.

For the $750-\mathrm{kW}$ plan at $13.8 \mathrm{kV}$, losses at peak generation of $49.5 \mathrm{MW}$ are $1.4 \mathrm{MW}$, or $2.9 \%$ of generation. These are the $\mathrm{I}^{2} \mathrm{R}$ losses on the collector system. Thus, the power delivered to the utility $115-\mathrm{kV}$ bus is approximately $48.1 \mathrm{MW}$. At $24.9 \mathrm{kV}$, losses are $1.20 \mathrm{MW}$, or $2.4 \%$. Approximately $48.3 \mathrm{MW}$ is delivered. Table $\mathrm{C} 1$ summarizes the power delivery for the remaining plans. No analysis has been made with regard to economic optimization of the collector system for losses. This can be done as an additional design effort. We do not expect it would have a significant impact on the costs.

The power flow studies were conducted on the basis of holding one-per-unit voltage at the generators, assuming that each wind generator can regulate VARs between lagging and leading $95 \%$ power factor. The power flow calculation confirmed that this was a valid assumption. 
With each generator providing VARs to regulate its own terminal voltage, the overall collector system VAR requirement was determined. At full generation, the VAR losses on the collector system were approximately $10 \mathrm{MVAR}$. Thus, as the wind generation varies from $0 \mathrm{MW}$ to 50 MW, the VAR losses on the collector system vary from 0 to $10 \mathrm{MVAR}$. If this were not corrected, it would be supplied by the utility. A fluctuating VAR load of 0 to 10 MVAR may cause voltage-regulating problems on the utility $115-\mathrm{kV}$ system. We assumed that the utility would require that the net generation to the utility be near unity power factor. We believe the best method for meeting this requirement would be to install a power electronically switched capacitor bank. We included the cost for this device in our substation cost estimate.

Notwithstanding the need to further optimize the design, the studies demonstrate that a $15-\mathrm{kV}$ class collector system is a viable concept for a wind farm comprising $750-\mathrm{kW}$ generators. However, based on the cost estimates we assembled, a $25-\mathrm{kV}$ system will cost less than a $15-\mathrm{kV}$ system. Furthermore, as can be seen from Table E1 in Appendix E, for the remaining turbine sizes, the $25-\mathrm{kV}$ plans were determined to be less costly than the $35-\mathrm{kV}$ plans. 
Table C1

WindPACT Turbine Design Scaling Studies

Plan Comparisons

\begin{tabular}{|c|c|c|c|c|c|c|c|c|c|}
\hline \multicolumn{2}{|c|}{ Plant Sizes } & \multicolumn{3}{|c|}{ Generation System } & \multicolumn{2}{c|}{ System Losses } & \multicolumn{3}{c|}{ Power Delivered @ 115 kV } \\
\hline kW & kV & MW & MVar & pf & Loss(MW) & MVar & MW & MVar & pf \\
\hline \multirow{2}{*}{$\mathbf{7 5 0}$} & 15 & 49.50 & -0.41 & $100.0 \%$ & 1.41 & 11.01 & 48.09 & -11.42 & $97.3 \%$ \\
& 25 & 49.50 & -1.70 & $99.9 \%$ & 1.20 & 9.06 & 48.30 & -10.76 & $97.6 \%$ \\
\hline \multirow{2}{*}{2500} & 25 & 50.00 & 0.01 & $100.0 \%$ & 1.15 & 10.54 & 48.86 & -10.53 & $97.8 \%$ \\
& 35 & 50.00 & 0.48 & $100.0 \%$ & 1.17 & 11.19 & 48.83 & -10.71 & $97.7 \%$ \\
\hline \multirow{2}{*}{5000} & 25 & 50.00 & 2.02 & $99.9 \%$ & 1.46 & 13.99 & 48.54 & -11.97 & $97.1 \%$ \\
& 35 & 50.00 & -0.89 & $100.0 \%$ & 1.29 & 10.25 & 48.72 & -11.14 & $97.5 \%$ \\
\hline \multirow{2}{*}{$\mathbf{1 0 0 0 0 0}$} & 25 & 50.00 & 0.83 & $100.0 \%$ & 1.28 & 12.09 & 48.72 & -11.26 & $97.4 \%$ \\
& 35 & 50.00 & 1.01 & $100.0 \%$ & 1.06 & 11.26 & 48.94 & -10.25 & $97.9 \%$ \\
\hline
\end{tabular}

C1 - 1 
Table C2.1

Mission Wind Farm - 750 kW / 15 kV WindPACT Turbine Design Scaling Studies

Power Flow Bus Report (100\% Generation, 49.5 MW)

\begin{tabular}{|c|c|c|c|c|c|}
\hline \multicolumn{2}{|c|}{ Bus } & \multirow{2}{*}{$\begin{array}{c}\text { Base } \\
\mathrm{kV}\end{array}$} & \multirow{2}{*}{$\begin{array}{c}\text { Voltage } \\
\text { P.U. }\end{array}$} & \multicolumn{2}{|c|}{ Generation } \\
\hline Number & Name & & & $\mathbf{P}(\mathbf{M W})$ & $\mathrm{Q}$ (MVAr) \\
\hline & Mission_115 & 115 & 1.0000 & -48.087 & 11.4232 \\
\hline 201 & Turbine_01 & 0.48 & 1.0000 & 0.75 & -0.1062 \\
\hline 202 & Turbine_02 & 0.48 & 1.0000 & 0.75 & -0.1010 \\
\hline 203 & Turbine_03 & 0.48 & 1.0000 & 0.75 & -0.0906 \\
\hline 204 & Turbine_04 & 0.48 & 1.0000 & 0.75 & -0.0750 \\
\hline 205 & Turbine_05 & 0.48 & 1.0000 & 0.75 & -0.0542 \\
\hline 206 & Turbine_06 & 0.48 & 1.0000 & 0.75 & -0.0280 \\
\hline 207 & Turbine_07 & 0.48 & 1.0000 & 0.75 & -0.0126 \\
\hline 208 & Turbine_08 & 0.48 & 1.0000 & 0.75 & 0.0056 \\
\hline 209 & Turbine_09 & 0.48 & 1.0000 & 0.75 & 0.0264 \\
\hline 210 & Turbine_10 & 0.48 & 1.0000 & 0.75 & 0.0500 \\
\hline 211 & Turbine_11 & 0.48 & 1.0000 & 0.75 & 0.0614 \\
\hline 212 & Turbine_12 & 0.48 & 1.0000 & 0.75 & 0.0606 \\
\hline 213 & Turbine_13 & 0.48 & 1.0000 & 0.75 & 0.0485 \\
\hline 214 & Turbine_14 & 0.48 & 1.0000 & 0.75 & 0.0227 \\
\hline 215 & Turbine_15 & 0.48 & 1.0000 & 0.75 & -0.0003 \\
\hline 216 & Turbine_16 & 0.48 & 1.0000 & 0.75 & -0.0207 \\
\hline 217 & Turbine_17 & 0.48 & 1.0000 & 0.75 & -0.0384 \\
\hline 218 & Turbine_18 & 0.48 & 1.0000 & 0.75 & -0.0693 \\
\hline 219 & Turbine_19 & 0.48 & 1.0000 & 0.75 & -0.0951 \\
\hline 220 & Turbine_20 & 0.48 & 1.0000 & 0.75 & -0.1156 \\
\hline 221 & Turbine_21 & 0.48 & 1.0000 & 0.75 & -0.1309 \\
\hline 222 & Turbine_22 & 0.48 & 1.0000 & 0.75 & -0.1411 \\
\hline 223 & Turbine_23 & 0.48 & 1.0000 & 0.75 & -0.1463 \\
\hline 224 & Turbine_24 & 0.48 & 1.0000 & 0.75 & 0.0166 \\
\hline 225 & Turbine_25 & 0.48 & 1.0000 & 0.75 & 0.0220 \\
\hline 226 & Turbine_26 & 0.48 & 1.0000 & 0.75 & 0.0329 \\
\hline 227 & Turbine_27 & 0.48 & 1.0000 & 0.75 & 0.0492 \\
\hline 228 & Turbine_28 & 0.48 & 1.0000 & 0.75 & 0.0710 \\
\hline 229 & Turbine_29 & 0.48 & 1.0000 & 0.75 & 0.0984 \\
\hline 230 & Turbine_30 & 0.48 & 1.0000 & 0.75 & 0.1055 \\
\hline 231 & Turbine_31 & 0.48 & 1.0000 & 0.75 & 0.0882 \\
\hline 232 & Turbine_32 & 0.48 & 1.0000 & 0.75 & 0.0723 \\
\hline 233 & Turbine_33 & 0.48 & 1.0000 & 0.75 & 0.0577 \\
\hline 234 & Turbine_34 & 0.48 & 1.0000 & 0.75 & 0.0443 \\
\hline 235 & Turbine_35 & 0.48 & 1.0000 & 0.75 & 0.0322 \\
\hline 236 & Turbine_36 & 0.48 & 1.0000 & 0.75 & 0.0213 \\
\hline 237 & Turbine_37 & 0.48 & 1.0000 & 0.75 & -0.0017 \\
\hline 238 & Turbine_38 & 0.48 & 1.0000 & 0.75 & -0.0221 \\
\hline 239 & Turbine_39 & 0.48 & 1.0000 & 0.75 & -0.0398 \\
\hline 240 & Turbine_40 & 0.48 & 1.0000 & 0.75 & -0.0707 \\
\hline 241 & Turbine_41 & 0.48 & 1.0000 & 0.75 & -0.0964 \\
\hline 242 & Turbine_42 & 0.48 & 1.0000 & 0.75 & -0.1169 \\
\hline 243 & Turbine_43 & 0.48 & 1.0000 & 0.75 & -0.1323 \\
\hline 244 & Turbine_44 & 0.48 & 1.0000 & 0.75 & -0.1425 \\
\hline
\end{tabular}


Table C2.1

Mission Wind Farm - 750 kW / 15 kV WindPACT Turbine Design Scaling Studies

Power Flow Bus Report (100\% Generation, 49.5 MW)

\begin{tabular}{|c|c|c|c|c|c|}
\hline \multicolumn{2}{|r|}{ Bus } & \multirow{2}{*}{$\begin{array}{c}\text { Base } \\
\text { kV } \\
\end{array}$} & \multirow{2}{*}{\begin{tabular}{c|} 
Voltage \\
P.U. \\
\end{tabular}} & Generation & eration \\
\hline Number & Name & & & $\mathbf{P}(\mathbf{M W})$ & Q(MVAr) \\
\hline \multicolumn{2}{|c|}{245 Turbine_45 } & 0.48 & 1.0000 & 0.75 & -0.1476 \\
\hline \multicolumn{2}{|c|}{246 Turbine 46} & 0.48 & 1.0000 & 0.75 & 0.1386 \\
\hline \multicolumn{2}{|c|}{247 Turbine_47 } & 0.48 & 1.0000 & 0.75 & 0.1443 \\
\hline \multicolumn{2}{|c|}{248 Turbine_48 } & 0.48 & 1.0000 & 0.75 & 0.1556 \\
\hline \multicolumn{2}{|c|}{249 Turbine_49 } & 0.48 & 1.0000 & 0.75 & 0.1472 \\
\hline \multicolumn{2}{|c|}{250 Turbine_50 } & 0.48 & 1.0000 & 0.75 & 0.1331 \\
\hline \multicolumn{2}{|c|}{251 Turbine_51 } & 0.48 & 1.0000 & 0.75 & 0.1199 \\
\hline \multicolumn{2}{|c|}{252 Turbine_52 } & 0.48 & 1.0000 & 0.75 & 0.1077 \\
\hline \multicolumn{2}{|c|}{253 Turbine_53 } & 0.48 & 1.0000 & 0.75 & 0.0916 \\
\hline \multicolumn{2}{|c|}{254 Turbine_54 } & 0.48 & 1.0000 & 0.75 & 0.0767 \\
\hline \multicolumn{2}{|c|}{255 Turbine_55 } & 0.48 & 1.0000 & 0.75 & 0.0631 \\
\hline \multicolumn{2}{|c|}{256 Turbine_56 } & 0.48 & 1.0000 & 0.75 & 0.0508 \\
\hline \multicolumn{2}{|c|}{257 Turbine_57 } & 0.48 & 1.0000 & 0.75 & 0.0287 \\
\hline \multicolumn{2}{|c|}{258 Turbine_58 } & 0.48 & 1.0000 & 0.75 & 0.0055 \\
\hline \multicolumn{2}{|c|}{259 Turbine_59 } & 0.48 & 1.0000 & 0.75 & -0.0151 \\
\hline \multicolumn{2}{|c|}{260 Turbine_60 } & 0.48 & 1.0000 & 0.75 & -0.0329 \\
\hline \multicolumn{2}{|c|}{261 Turbine_61 } & 0.48 & 1.0000 & 0.75 & -0.0481 \\
\hline \multicolumn{2}{|c|}{262 Turbine_62 } & 0.48 & 1.0000 & 0.75 & -0.0739 \\
\hline 263 & Turbine_63 & 0.48 & 1.0000 & 0.75 & -0.0945 \\
\hline 264 & Turbine_64 & 0.48 & 1.0000 & 0.75 & -0.1253 \\
\hline 265 & Turbine_65 & 0.48 & 1.0000 & 0.75 & -0.1356 \\
\hline 266 & Turbine_66 & 0.48 & 1.0000 & 0.75 & -0.1407 \\
\hline & & & & $\begin{array}{r}49.50 \\
1.41\end{array}$ & $\begin{array}{l}-0.4120 \text { Total Wind Generation } \\
11.01 \text { Total Losses }\end{array}$ \\
\hline & Mission_13.8 & 13.8 & 0.9845 & & \\
\hline 10 & Riser_A & 13.8 & 0.9919 & & \\
\hline 15 & Riser_B & 13.8 & 0.9895 & & \\
\hline 20 & Riser_C & 13.8 & 0.9890 & & \\
\hline 25 & Riser_D & 13.8 & 0.9862 & & \\
\hline 30 & Riser_E & 13.8 & 0.9868 & & \\
\hline 101 & Wind_01 & 13.8 & 1.0023 & & \\
\hline 102 & Wind_02 & 13.8 & 1.0020 & & \\
\hline 103 & Wind_03 & 13.8 & 1.0014 & & \\
\hline 104 & Wind_04 & 13.8 & 1.0005 & & \\
\hline 105 & Wind_05 & 13.8 & 0.9993 & & \\
\hline 106 & Wind_06 & 13.8 & 0.9978 & & \\
\hline 107 & Wind_07 & 13.8 & 0.9969 & & \\
\hline 108 & Wind_08 & 13.8 & 0.9958 & & \\
\hline 109 & Wind_09 & 13.8 & 0.9946 & & \\
\hline 110 & Wind_10 & 13.8 & 0.9933 & & \\
\hline 111 & Wind_-11 & 13.8 & 0.9926 & & \\
\hline 112 & Wind_12 & 13.8 & 0.9926 & & \\
\hline 113 & Wind_13 & 13.8 & 0.9933 & & \\
\hline 114 & Wind_14 & 13.8 & 0.9948 & & \\
\hline 115 & Wind_-15 & 13.8 & 0.9962 & & \\
\hline 116 & Wind_16 & 13.8 & 0.9973 & & \\
\hline 117 & Wind_17 & 13.8 & 0.9984 & & \\
\hline 118 & Wind_18 & 13.8 & 1.0001 & & \\
\hline
\end{tabular}


Table C2.1

Mission Wind Farm - 750 kW / 15 kV WindPACT Turbine Design Scaling Studies

Power Flow Bus Report (100\% Generation, 49.5 MW)

\begin{tabular}{|c|c|c|c|c|}
\hline \multicolumn{2}{|r|}{ Bus } & \multirow{2}{*}{$\frac{\text { Base }}{\mathrm{kV}}$} & \multirow{2}{*}{\begin{tabular}{c|} 
Voltage \\
P.U. \\
\end{tabular}} & Generation \\
\hline Number & Name & & & \begin{tabular}{l|l}
$P(M W)$ & $Q(M V A r)$ \\
\end{tabular} \\
\hline 119 & Wind_19 & 13.8 & 1.0016 & \\
\hline 120 & Wind_20 & 13.8 & 1.0028 & \\
\hline 121 & Wind_21 & 13.8 & 1.0037 & \\
\hline 122 & Wind_22 & 13.8 & 1.0043 & \\
\hline 123 & Wind_23 & 13.8 & 1.0046 & \\
\hline 124 & Wind_24 & 13.8 & 0.9952 & \\
\hline 125 & Wind_25 & 13.8 & 0.9949 & \\
\hline 126 & Wind_26 & 13.8 & 0.9942 & \\
\hline 127 & Wind_27 & 13.8 & 0.9933 & \\
\hline 128 & Wind_28 & 13.8 & 0.9920 & \\
\hline 129 & Wind_29 & 13.8 & 0.9905 & \\
\hline 130 & Wind_30 & 13.8 & 0.9901 & \\
\hline 131 & Wind_31 & 13.8 & 0.9910 & \\
\hline 132 & Wind_32 & 13.8 & 0.9920 & \\
\hline 133 & Wind_33 & 13.8 & 0.9928 & \\
\hline 134 & Wind_34 & 13.8 & 0.9936 & \\
\hline 135 & Wind_35 & 13.8 & 0.9943 & \\
\hline 136 & Wind_36 & 13.8 & 0.9949 & \\
\hline 137 & Wind_37 & 13.8 & 0.9962 & \\
\hline 138 & Wind_38 & 13.8 & 0.9974 & \\
\hline 139 & Wind_39 & 13.8 & 0.9984 & \\
\hline 140 & Wind_40 & 13.8 & 1.0002 & \\
\hline 141 & Wind_41 & 13.8 & 1.0017 & \\
\hline 142 & Wind_42 & 13.8 & 1.0029 & \\
\hline 143 & Wind_43 & 13.8 & 1.0038 & \\
\hline 144 & Wind_44 & 13.8 & 1.0044 & \\
\hline 145 & Wind_45 & 13.8 & 1.0047 & \\
\hline 146 & Wind_46 & 13.8 & 0.9881 & \\
\hline 147 & Wind_47 & 13.8 & 0.9878 & \\
\hline 148 & Wind_48 & 13.8 & 0.9872 & \\
\hline 149 & Wind_49 & 13.8 & 0.9876 & \\
\hline 150 & Wind_50 & 13.8 & 0.9885 & \\
\hline 151 & Wind_51 & 13.8 & 0.9892 & \\
\hline 152 & Wind_52 & 13.8 & 0.9899 & \\
\hline 153 & Wind_53 & 13.8 & 0.9909 & \\
\hline 154 & Wind_54 & 13.8 & 0.9917 & \\
\hline 155 & Wind_55 & 13.8 & 0.9925 & \\
\hline 156 & Wind_56 & 13.8 & 0.9932 & \\
\hline 157 & Wind_57 & 13.8 & 0.9945 & \\
\hline 158 & Wind_58 & 13.8 & 0.9958 & \\
\hline 159 & Wind_59 & 13.8 & 0.9970 & \\
\hline 160 & Wind_60 & 13.8 & 0.9980 & \\
\hline 161 & Wind_61 & 13.8 & 0.9989 & \\
\hline 162 & Wind_62 & 13.8 & 1.0004 & \\
\hline 163 & Wind_63 & 13.8 & 1.0016 & \\
\hline 164 & Wind_64 & 13.8 & 1.0034 & \\
\hline 165 & Wind_65 & 13.8 & 1.0040 & \\
\hline 166 & Wind_66 & 13.8 & 1.0043 & \\
\hline
\end{tabular}


Table C2.2

Mission Wind Farm - 750 kW / 15 kV

WindPACT Turbine Design Scaling Studies

Power Flow Bus Report (100\% Generation, 49.5 MW)

\begin{tabular}{|c|c|c|c|c|c|c|c|c|}
\hline From Bus & To Bus & Ckt & MW & MVar & MVA & AMPS & Norm & \%Norm \\
\hline 130 Wind_30 & 20 Riser_C & 1 & 12.07 & -0.78 & 12.09 & 511 & 12 & 100.8 \\
\hline 1 Mission 115 & 2 Mission 13.8 & 1 & -48.09 & 11.43 & 49.43 & 2068 & 50 & 98.9 \\
\hline 149 Wind 49 & 150 Wind 50 & 1 & -13.25 & 0.03 & 13.25 & 561 & 14 & 94.7 \\
\hline 113 Wind 13 & 114 Wind 14 & 1 & -7.48 & 1.07 & 7.55 & 318 & 8 & 94.4 \\
\hline 30 Riser_E & 2 Mission_13.8 & 1 & 25.10 & -1.47 & 25.15 & 1066 & 27 & 93.1 \\
\hline 139 Wind_39 & 140 Wind_ $4 \overline{0}$ & 1 & -4.50 & 0.93 & 4.60 & 192 & 5 & 91.9 \\
\hline 149 Wind_49 & 30 Riser_E & 1 & 12.77 & -1.00 & 12.81 & 543 & 14 & 91.5 \\
\hline 131 Wind_31 & 132 Wind_32 & 1 & -10.92 & 0.62 & 10.94 & 461 & 12 & 91.1 \\
\hline 152 Wind_52 & 153 Wind_53 & 1 & -10.82 & 0.50 & 10.83 & 457 & 12 & 90.2 \\
\hline 130 Wind_30 & 131 Wind_31 & 1 & -10.51 & 1.46 & 10.61 & 448 & 12 & 88.4 \\
\hline 25 Riser_D & 2 Mission_13.8 & 1 & 23.52 & -2.20 & 23.62 & 1002 & 27 & 87.5 \\
\hline 114 Wind_14 & 115 Wind_1 $1 \overline{5}$ & 1 & -6.94 & 0.89 & 7.00 & 294 & 8 & 87.5 \\
\hline 117 Wind 17 & 118 Wind 18 & 1 & -4.27 & 0.95 & 4.37 & 183 & 5 & 87.4 \\
\hline 136 Wind_36 & 137 Wind 37 & 1 & -6.56 & 1.12 & 6.66 & 280 & 8 & 83.2 \\
\hline 157 Wind_57 & 158 Wind_58 & 1 & -6.51 & 0.98 & 6.58 & 276 & 8 & 82.2 \\
\hline 150 Wind_50 & 151 Wind 51 & 1 & -11.33 & 1.01 & 11.38 & 481 & 14 & 81.3 \\
\hline 109 Wind_09 & 110 Wind_10 & 1 & 6.44 & -0.83 & 6.49 & 273 & 8 & 81.2 \\
\hline 15 Riser_B & 25 Riser_D & 1 & 21.42 & -2.04 & 21.51 & 910 & 27 & 79.7 \\
\hline 151 Wind_51 & 152 Wind_52 & 1 & -11.09 & 0.77 & 11.12 & 470 & 14 & 79.4 \\
\hline 132 Wind_32 & 133 Wind_33 & 1 & -9.36 & 1.29 & 9.45 & 398 & 12 & 78.7 \\
\hline 153 Wind_53 & 154 Wind_54 & 1 & -9.26 & 1.17 & 9.33 & 394 & 12 & 77.8 \\
\hline 118 Wind_18 & 119 Wind_19 & 1 & -3.76 & 0.82 & 3.85 & 161 & 5 & 77 \\
\hline 145 Wind_45 & 245 Turbine_45 & 1 & -0.75 & 0.18 & 0.77 & 920 & 1 & 76.8 \\
\hline 123 Wind_23 & 223 Turbine_23 & 1 & -0.75 & 0.18 & 0.77 & 919 & 1 & 76.8 \\
\hline 137 Wind_37 & 138 Wind $3 \overline{8}$ & 1 & -6.06 & 1.01 & 6.14 & 258 & 8 & 76.7 \\
\hline 140 Wind_40 & 141 Wind_41 & 1 & -3.76 & 0.78 & 3.84 & 160 & 5 & 76.7 \\
\hline 144 Wind_44 & 244 Turbine_44 & 1 & -0.75 & 0.18 & 0.77 & 918 & 1 & 76.7 \\
\hline 122 Wind_22 & 222 Turbine_22 & 1 & -0.75 & 0.17 & 0.77 & 918 & 1 & 76.7 \\
\hline 166 Wind_66 & 266 Turbine_66 & 1 & -0.75 & 0.17 & 0.77 & 918 & 1 & 76.7 \\
\hline 148 Wind_48 & 248 Turbine_48 & 1 & -0.75 & -0.15 & 0.77 & 921 & 1 & 76.6 \\
\hline 165 Wind_65 & 265 Turbine_65 & 1 & -0.75 & 0.17 & 0.77 & 917 & 1 & 76.5 \\
\hline 149 Wind 49 & 249 Turbine 49 & 1 & -0.75 & -0.15 & 0.76 & 920 & 1 & 76.5 \\
\hline 121 Wind_21 & 221 Turbine_21 & 1 & -0.75 & 0.16 & 0.76 & 916 & 1 & 76.4 \\
\hline 143 Wind 43 & 243 Turbine_-43 & 1 & -0.75 & 0.17 & 0.76 & 916 & 1 & 76.4 \\
\hline 147 Wind_47 & 247 Turbine_47 & 1 & -0.75 & -0.14 & 0.76 & 919 & 1 & 76.4 \\
\hline 164 Wind_64 & 264 Turbine_64 & 1 & -0.75 & 0.16 & 0.76 & 915 & 1 & 76.3 \\
\hline 146 Wind_46 & 246 Turbine_46 & 1 & -0.75 & -0.14 & 0.76 & 918 & 1 & 76.3 \\
\hline 158 Wind_58 & 159 Wind_59 & 1 & -6.02 & 0.93 & 6.10 & 256 & 8 & 76.2 \\
\hline 142 Wind_42 & 242 Turbine_42 & 1 & -0.75 & 0.15 & 0.76 & 913 & 1 & 76.2 \\
\hline 150 Wind_50 & 250 Turbine_50 & 1 & -0.75 & -0.13 & 0.76 & 916 & 1 & 76.1 \\
\hline 120 Wind_20 & 220 Turbine_20 & 1 & -0.75 & 0.15 & 0.76 & 913 & 1 & 76.1 \\
\hline 151 Wind 51 & 251 Turbine_ 51 & 1 & -0.75 & -0.12 & 0.76 & 914 & 1 & 76 \\
\hline 161 Wind_61 & 162 Wind_6z & 1 & -3.73 & 0.69 & 3.80 & 159 & 5 & 75.9 \\
\hline 101 Wind_01 & 201 Turbine_01 & 1 & -0.75 & 0.14 & 0.76 & 911 & 1 & 75.9 \\
\hline 102 Wind_02 & 202 Turbine_02 & 1 & -0.75 & 0.13 & 0.76 & 910 & 1 & 75.8 \\
\hline 152 Wind_52 & 252 Turbine_52 & 1 & -0.75 & -0.11 & 0.76 & 911 & 1 & 75.8 \\
\hline 141 Wind_41 & 241 Turbine_41 & 1 & -0.75 & 0.13 & 0.76 & 910 & 1 & 75.8 \\
\hline
\end{tabular}


Table C2.2

Mission Wind Farm - 750 kW / 15 kV

WindPACT Turbine Design Scaling Studies

Power Flow Bus Report (100\% Generation, 49.5 MW)

\begin{tabular}{|c|c|c|c|c|c|c|c|c|}
\hline From Bus & To Bus & Ckt & MW & MVar & MVA & AMPS & Norm & \%Norm \\
\hline 130 Wind_30 & 230 Turbine_30 & 1 & -0.75 & -0.10 & 0.76 & 911 & 1 & 75.7 \\
\hline 163 Wind_63 & 263 Turbine_63 & 1 & -0.75 & 0.13 & 0.76 & 909 & 1 & 75.7 \\
\hline 119 Wind_19 & 219 Turbine_19 & 1 & -0.75 & 0.13 & 0.76 & 909 & 1 & 75.7 \\
\hline 103 Wind_03 & 203 Turbine_03 & 1 & -0.75 & 0.12 & 0.76 & 909 & 1 & 75.7 \\
\hline 129 Wind_29 & 229 Turbine_29 & 1 & -0.75 & -0.10 & 0.76 & 910 & 1 & 75.6 \\
\hline 153 Wind_53 & 253 Turbine_53 & 1 & -0.75 & -0.09 & 0.76 & 909 & 1 & 75.6 \\
\hline 133 Wind_33 & 134 Wind_34 & 1 & -9.02 & 0.85 & 9.06 & 381 & 12 & 75.5 \\
\hline 131 Wind_31 & 231 Turbiñe_31 & 1 & -0.75 & -0.09 & 0.76 & 908 & 1 & 75.5 \\
\hline 154 Wind_54 & 155 Wind_5 $5 \overline{5}$ & 1 & -9.00 & 0.84 & 9.04 & 381 & 12 & 75.4 \\
\hline 162 Wind_62 & 262 Turbine_e_62 & 1 & -0.75 & 0.11 & 0.75 & 907 & 1 & 75.4 \\
\hline 154 Wind_54 & 254 Turbine_54 & 1 & -0.75 & -0.08 & 0.75 & 907 & 1 & 75.4 \\
\hline 104 Wind_04 & 204 Turbine_04 & 1 & -0.75 & 0.11 & 0.75 & 906 & 1 & 75.4 \\
\hline 128 Wind_28 & 228 Turbine_28 & 1 & -0.75 & -0.07 & 0.75 & 907 & 1 & 75.4 \\
\hline 140 Wind_40 & 240 Turbine_40 & 1 & -0.75 & 0.10 & 0.75 & 906 & 1 & 75.3 \\
\hline 132 Wind_32 & 232 Turbine_32 & 1 & -0.75 & -0.07 & 0.75 & 906 & 1 & 75.3 \\
\hline 118 Wind_18 & 218 Turbine_18 & 1 & -0.75 & 0.10 & 0.75 & 906 & 1 & 75.3 \\
\hline 112 Wind_12 & 212 Turbine_12 & 1 & -0.75 & -0.06 & 0.75 & 905 & 1 & 75.3 \\
\hline 111 Wind_11 & 211 Turbine_11 & 1 & -0.75 & -0.06 & 0.75 & 905 & 1 & 75.3 \\
\hline 155 Wind_55 & 255 Turbine_55 & 1 & -0.75 & -0.06 & 0.75 & 905 & 1 & 75.3 \\
\hline 133 Wind_33 & 233 Turbine_33 & 1 & -0.75 & -0.06 & 0.75 & 905 & 1 & 75.2 \\
\hline 105 Wind_05 & 205 Turbine_05 & 1 & -0.75 & 0.05 & 0.75 & 905 & 1 & 75.2 \\
\hline 156 Wind_56 & 256 Turbine_56 & 1 & -0.75 & -0.05 & 0.75 & 904 & 1 & 75.2 \\
\hline 127 Wind_27 & 227 Turbine_27 & 1 & -0.75 & -0.05 & 0.75 & 904 & 1 & 75.2 \\
\hline 113 Wind_13 & 213 Turbine_13 & 1 & -0.75 & -0.05 & 0.75 & 904 & 1 & 75.2 \\
\hline 110 Wind_-10 & 210 Turbine_10 & 1 & -0.75 & -0.05 & 0.75 & 904 & 1 & 75.2 \\
\hline 134 Wind_34 & 234 Turbine_34 & 1 & -0.75 & -0.04 & 0.75 & 904 & 1 & 75.1 \\
\hline 139 Wind_39 & 239 Turbine_39 & 1 & -0.75 & 0.04 & 0.75 & 904 & 1 & 75.1 \\
\hline 161 Wind_61 & 261 Turbine_61 & 1 & -0.75 & 0.05 & 0.75 & 904 & 1 & 75.1 \\
\hline 117 Wind_17 & 217 Turbine_17 & 1 & -0.75 & 0.04 & 0.75 & 903 & 1 & 75.1 \\
\hline 126 Wind_26 & 226 Turbine_26 & 1 & -0.75 & -0.03 & 0.75 & 903 & 1 & 75.1 \\
\hline 135 Wind_35 & 235 Turbine_35 & 1 & -0.75 & -0.03 & 0.75 & 903 & 1 & 75.1 \\
\hline 160 Wind_60 & 260 Turbine_60 & 1 & -0.75 & 0.03 & 0.75 & 903 & 1 & 75.1 \\
\hline 136 Wind_36 & 236 Turbine_36 & 1 & -0.75 & -0.02 & 0.75 & 903 & 1 & 75 \\
\hline 109 Wind_09 & 209 Turbine_09 & 1 & -0.75 & -0.03 & 0.75 & 903 & 1 & 75 \\
\hline 114 Wind_14 & 214 Turbine_14 & 1 & -0.75 & -0.02 & 0.75 & 903 & 1 & 75 \\
\hline 106 Wind_06 & 206 Turbine_06 & 1 & -0.75 & 0.03 & 0.75 & 903 & 1 & 75 \\
\hline 116 Wind_16 & 216 Turbine_16 & 1 & -0.75 & 0.02 & 0.75 & 903 & 1 & 75 \\
\hline 157 Wind_57 & 257 Turbine_57 & 1 & -0.75 & -0.03 & 0.75 & 902 & 1 & 75 \\
\hline 138 Wind_38 & 238 Turbine_38 & 1 & -0.75 & 0.02 & 0.75 & 902 & 1 & 75 \\
\hline 125 Wind_25 & 225 Turbine_25 & 1 & -0.75 & -0.02 & 0.75 & 902 & 1 & 75 \\
\hline 137 Wind_37 & 237 Turbine_37 & 1 & -0.75 & 0.00 & 0.75 & 902 & 1 & 75 \\
\hline 115 Wind_15 & 215 Turbine_15 & 1 & -0.75 & 0.00 & 0.75 & 902 & 1 & 75 \\
\hline 107 Wind_07 & 207 Turbine_07 & 1 & -0.75 & 0.01 & 0.75 & 902 & 1 & 75 \\
\hline 158 Wind_58 & 258 Turbine_58 & 1 & -0.75 & -0.01 & 0.75 & 902 & 1 & 75 \\
\hline 108 Wind_08 & 208 Turbine_08 & 1 & -0.75 & -0.01 & 0.75 & 902 & 1 & 75 \\
\hline 124 Wind_24 & 224 Turbine_24 & 1 & -0.75 & -0.02 & 0.75 & 902 & 1 & 75 \\
\hline 159 Wind_59 & 259 Turbine_59 & 1 & -0.75 & 0.01 & 0.75 & 902 & 1 & 75 \\
\hline 105 Wind_05 & 106 Wind_0 $\overline{6}$ & 1 & 3.70 & -0.57 & 3.75 & 157 & 5 & 74.9 \\
\hline
\end{tabular}


Table C2.2

Mission Wind Farm - 750 kW / 15 kV

WindPACT Turbine Design Scaling Studies

Power Flow Bus Report (100\% Generation, 49.5 MW)

\begin{tabular}{|c|c|c|c|c|c|c|c|c|}
\hline From Bus & To Bus & Ckt & MW & MVar & MVA & AMPS & Norm & \%Norm \\
\hline 108 Wind_08 & 109 Wind_09 & 1 & 5.93 & -0.72 & 5.97 & 251 & 8 & 74.7 \\
\hline 115 Wind_15 & 116 Wind_16 & 1 & -5.65 & 1.17 & 5.77 & 242 & 8 & 72.1 \\
\hline 128 Wind_28 & 129 Wind_29 & 1 & 3.54 & 0.02 & 3.54 & 149 & 5 & 70.8 \\
\hline 112 Wind_12 & 10 Riser_A & 1 & 8.35 & -1.35 & 8.46 & 356 & 12 & 70.5 \\
\hline 116 Wind_16 & 117 Wind_17 & 1 & -5.52 & 0.82 & 5.58 & 234 & 8 & 69.7 \\
\hline 134 Wind_34 & 135 Wind_35 & 1 & -8.20 & 1.13 & 8.27 & 348 & 12 & 68.9 \\
\hline 112 Wind_12 & 113 Wind_13 & 1 & -8.18 & 1.12 & 8.26 & 348 & 12 & 68.8 \\
\hline 107 Wind_07 & 108 Wind_08 & 1 & 5.42 & -0.60 & 5.45 & 229 & 8 & 68.2 \\
\hline 155 Wind_55 & 156 Wind_56 & 1 & -8.09 & 1.01 & 8.16 & 344 & 12 & 68 \\
\hline 111 Wind_11 & 10 Riser_A & 1 & 7.91 & -0.78 & 7.95 & 335 & 12 & 66.3 \\
\hline 138 Wind_38 & 139 Wind_39 & 1 & -5.11 & 0.98 & 5.20 & 218 & 8 & 65 \\
\hline 110 Wind_10 & 111 Wind_11 & 1 & 7.75 & -0.55 & 7.77 & 327 & 12 & 64.7 \\
\hline 159 Wind_59 & 160 Wind_60 & 1 & -5.08 & 0.91 & 5.16 & 216 & 8 & 64.5 \\
\hline 10 Riser_A & 15 Riser_B & 1 & 17.05 & -1.69 & 17.13 & 723 & 27 & 63.4 \\
\hline 156 Wind_56 & 157 Wind_57 & 1 & -7.52 & 0.92 & 7.57 & 319 & 12 & 63.1 \\
\hline 141 Wind_41 & 142 Wind_42 & 1 & -3.02 & 0.66 & 3.09 & 129 & 5 & 61.7 \\
\hline 119 Wind_19 & 120 Wind_20 & 1 & -3.02 & 0.65 & 3.09 & 129 & 5 & 61.7 \\
\hline 162 Wind_62 & 163 Wind_63 & 1 & -3.01 & 0.65 & 3.08 & 129 & 5 & 61.6 \\
\hline 127 Wind_27 & 128 Wind_28 & 1 & 3.08 & 0.00 & 3.08 & 130 & 5 & 61.5 \\
\hline 135 Wind_35 & 136 Wind_36 & 1 & -7.19 & 1.18 & 7.29 & 307 & 12 & 60.8 \\
\hline 104 Wind_04 & 105 Wind_05 & 1 & 2.98 & -0.53 & 3.03 & 127 & 5 & 60.6 \\
\hline 160 Wind_60 & 161 Wind_61 & 1 & -4.56 & 0.80 & 4.63 & 194 & 8 & 57.9 \\
\hline 129 Wind_29 & 15 Riser_B & 1 & 4.61 & 0.16 & 4.62 & 195 & 8 & 57.7 \\
\hline 106 Wind_06 & 107 Wind_07 & 1 & 4.50 & -0.65 & 4.55 & 191 & 8 & 56.9 \\
\hline 142 Wind_-42 & 143 Wind_43 & 1 & -2.27 & 0.53 & 2.34 & 97 & 5 & 46.7 \\
\hline 120 Wind_20 & 121 Wind_21 & 1 & -2.26 & 0.49 & 2.32 & 97 & 5 & 46.3 \\
\hline 163 Wind_63 & 164 Wind_64 & 1 & -2.26 & 0.49 & 2.32 & 97 & 5 & 46.3 \\
\hline 148 Wind_48 & 25 Riser_D & 1 & 2.27 & 0.35 & 2.30 & 97 & 5 & 46 \\
\hline 103 Wind_03 & 104 Wind_04 & 1 & 2.23 & -0.37 & 2.26 & 95 & 5 & 45.2 \\
\hline 20 Riser_C & 30 Riser_E & 1 & 11.82 & -0.91 & 11.86 & 502 & 27 & 43.9 \\
\hline 126 Wind_26 & 127 Wind_27 & 1 & 2.14 & -0.05 & 2.14 & 90 & 5 & 42.9 \\
\hline 125 Wind_25 & 126 Wind_26 & 1 & 1.66 & 0.00 & 1.66 & 70 & 5 & 33.2 \\
\hline 121 Wind_21 & 122 Wind_22 & 1 & -1.52 & 0.37 & 1.56 & 65 & 5 & 31.3 \\
\hline 143 Wind 43 & 144 Wind 44 & 1 & -1.51 & 0.33 & 1.55 & 64 & 5 & 30.9 \\
\hline 164 Wind_64 & 165 Wind_65 & 1 & -1.51 & 0.33 & 1.55 & 64 & 5 & 30.9 \\
\hline 102 Wind_02 & 103 Wind_03 & 1 & 1.50 & -0.29 & 1.52 & 64 & 5 & 30.5 \\
\hline 147 Wind_47 & 148 Wind_48 & 1 & 1.37 & 0.19 & 1.38 & 59 & 5 & 27.6 \\
\hline 144 Wind_44 & 145 Wind_45 & 1 & -0.76 & 0.21 & 0.79 & 33 & 5 & 15.8 \\
\hline 165 Wind_65 & 166 Wind_66 & 1 & -0.76 & 0.17 & 0.77 & 32 & 5 & 15.5 \\
\hline 122 Wind_22 & 123 Wind_23 & 1 & -0.76 & 0.16 & 0.77 & 32 & 5 & 15.5 \\
\hline 101 Wind_01 & 102 Wind_02 & 1 & 0.74 & -0.12 & 0.76 & 32 & 5 & 15.1 \\
\hline 124 Wind_24 & 125 Wind_25 & 1 & 0.71 & 0.00 & 0.71 & 30 & 5 & 14.2 \\
\hline 146 Wind_46 & 147 Wind_47 & 1 & 0.69 & 0.07 & 0.69 & 29 & 5 & 13.9 \\
\hline
\end{tabular}


Table C3.1

Mission Wind Farm - 750 kW / 25 kV

WindPACT Turbine Design Scaling Studies

Power Flow Bus Report (100\% Generation, 49.5 MW)

\begin{tabular}{|c|c|c|c|c|c|}
\hline \multicolumn{2}{|r|}{ Bus } & \multirow{2}{*}{$\begin{array}{c}\text { Base } \\
\mathrm{kV}\end{array}$} & \multirow{2}{*}{\begin{tabular}{|c|} 
Voltage \\
P.U.
\end{tabular}} & \multicolumn{2}{|c|}{ Generation } \\
\hline Number & Name & & & $\mathrm{P}(\mathrm{MW})$ & Q(MVAr) \\
\hline & Mission_115 & 115 & 1.0000 & -48.298 & 10.7626 \\
\hline 201 & Turbine_01 & 0.48 & 1.0000 & 0.75 & -0.0852 \\
\hline 202 & Turbine_02 & 0.48 & 1.0000 & 0.75 & -0.0836 \\
\hline 203 & Turbine_03 & 0.48 & 1.0000 & 0.75 & -0.0803 \\
\hline 204 & Turbine_04 & 0.48 & 1.0000 & 0.75 & -0.0755 \\
\hline 205 & Turbine_05 & 0.48 & 1.0000 & 0.75 & -0.0691 \\
\hline 206 & Turbine_06 & 0.48 & 1.0000 & 0.75 & -0.0611 \\
\hline 207 & Turbine_07 & 0.48 & 1.0000 & 0.75 & -0.0514 \\
\hline 208 & Turbine_08 & 0.48 & 1.0000 & 0.75 & -0.0401 \\
\hline 209 & Turbine_09 & 0.48 & 1.0000 & 0.75 & -0.0272 \\
\hline 210 & Turbine_10 & 0.48 & 1.0000 & 0.75 & -0.0127 \\
\hline 211 & Turbine_11 & 0.48 & 1.0000 & 0.75 & 0.0035 \\
\hline 212 & Turbine_12 & 0.48 & 1.0000 & 0.75 & 0.0020 \\
\hline 213 & Turbine_13 & 0.48 & 1.0000 & 0.75 & -0.0157 \\
\hline 214 & Turbine_14 & 0.48 & 1.0000 & 0.75 & -0.0318 \\
\hline 215 & Turbine_15 & 0.48 & 1.0000 & 0.75 & -0.0462 \\
\hline 216 & Turbine_16 & 0.48 & 1.0000 & 0.75 & -0.0590 \\
\hline 217 & Turbine_17 & 0.48 & 1.0000 & 0.75 & -0.0702 \\
\hline 218 & Turbine_18 & 0.48 & 1.0000 & 0.75 & -0.0798 \\
\hline 219 & Turbine_19 & 0.48 & 1.0000 & 0.75 & -0.0878 \\
\hline 220 & Turbine_20 & 0.48 & 1.0000 & 0.75 & -0.0942 \\
\hline 221 & Turbine_21 & 0.48 & 1.0000 & 0.75 & -0.0990 \\
\hline 222 & Turbine_22 & 0.48 & 1.0000 & 0.75 & -0.1021 \\
\hline 223 & Turbine_23 & 0.48 & 1.0000 & 0.75 & -0.1037 \\
\hline 224 & Turbine_24 & 0.48 & 1.0000 & 0.75 & 0.0067 \\
\hline 225 & Turbine_25 & 0.48 & 1.0000 & 0.75 & 0.0084 \\
\hline 226 & Turbine_26 & 0.48 & 1.0000 & 0.75 & 0.0117 \\
\hline 227 & Turbine_27 & 0.48 & 1.0000 & 0.75 & 0.0167 \\
\hline 228 & Turbine_28 & 0.48 & 1.0000 & 0.75 & 0.0233 \\
\hline 229 & Turbine_29 & 0.48 & 1.0000 & 0.75 & 0.0317 \\
\hline 230 & Turbine_30 & 0.48 & 1.0000 & 0.75 & 0.0417 \\
\hline 231 & Turbine_31 & 0.48 & 1.0000 & 0.75 & 0.0476 \\
\hline 232 & Turbine_32 & 0.48 & 1.0000 & 0.75 & 0.0408 \\
\hline 233 & Turbine_33 & 0.48 & 1.0000 & 0.75 & 0.0281 \\
\hline 234 & Turbine_34 & 0.48 & 1.0000 & 0.75 & 0.0162 \\
\hline 235 & Turbine_35 & 0.48 & 1.0000 & 0.75 & 0.0052 \\
\hline 236 & Turbine_36 & 0.48 & 1.0000 & 0.75 & -0.0051 \\
\hline 237 & Turbine_37 & 0.48 & 1.0000 & 0.75 & -0.0242 \\
\hline 238 & Turbine_38 & 0.48 & 1.0000 & 0.75 & -0.0418 \\
\hline 239 & Turbine_39 & 0.48 & 1.0000 & 0.75 & -0.0577 \\
\hline 240 & Turbine_40 & 0.48 & 1.0000 & 0.75 & -0.0720 \\
\hline 241 & Turbine_41 & 0.48 & 1.0000 & 0.75 & -0.0847 \\
\hline 242 & Turbine_42 & 0.48 & 1.0000 & 0.75 & -0.0958 \\
\hline 243 & Turbine_43 & 0.48 & 1.0000 & 0.75 & -0.1053 \\
\hline 244 & Turbine_44 & 0.48 & 1.0000 & 0.75 & -0.1132 \\
\hline 245 & Turbine_45 & 0.48 & 1.0000 & 0.75 & -0.1195 \\
\hline 246 & Turbine_46 & 0.48 & 1.0000 & 0.75 & -0.1242 \\
\hline 247 & Turbine_47 & 0.48 & 1.0000 & 0.75 & -0.1274 \\
\hline 248 & Turbine_48 & 0.48 & 1.0000 & 0.75 & -0.1289 \\
\hline 249 & Turbine_49 & 0.48 & 1.0000 & 0.75 & 0.1190 \\
\hline 250 & Turbine_50 & 0.48 & 1.0000 & 0.75 & 0.1199 \\
\hline 251 & Turbine_51 & 0.48 & 1.0000 & 0.75 & 0.1217 \\
\hline 252 & Turbine_52 & 0.48 & 1.0000 & 0.75 & 0.1119 \\
\hline
\end{tabular}


Table C3.1

Mission Wind Farm - 750 kW / 25 kV

WindPACT Turbine Design Scaling Studies

Power Flow Bus Report (100\% Generation, 49.5 MW)

\begin{tabular}{|c|c|c|c|c|c|}
\hline \multicolumn{2}{|r|}{ Bus } & \multirow{2}{*}{$\begin{array}{c}\text { Base } \\
\mathrm{kV}\end{array}$} & \multirow{2}{*}{\begin{tabular}{|c|} 
Voltage \\
P.U.
\end{tabular}} & Generation & eration \\
\hline Number & Name & & & $P(M W)$ & Q(MVAr) \\
\hline \multicolumn{2}{|c|}{253 Turbine_53 } & 0.48 & 1.0000 & 0.75 & 0.1002 \\
\hline \multicolumn{2}{|c|}{254 Turbine 54} & 0.48 & 1.0000 & 0.75 & 0.0895 \\
\hline \multicolumn{2}{|c|}{255 Turbine_55 } & 0.48 & 1.0000 & 0.75 & 0.0697 \\
\hline \multicolumn{2}{|c|}{256 Turbine_56 } & 0.48 & 1.0000 & 0.75 & 0.0516 \\
\hline \multicolumn{2}{|c|}{257 Turbine 57} & 0.48 & 1.0000 & 0.75 & 0.0188 \\
\hline \multicolumn{2}{|c|}{258 Turbine_58 } & 0.48 & 1.0000 & 0.75 & 0.0041 \\
\hline \multicolumn{2}{|c|}{259 Turbine_59 } & 0.48 & 1.0000 & 0.75 & -0.0090 \\
\hline \multicolumn{2}{|c|}{260 Turbine_60 } & 0.48 & 1.0000 & 0.75 & -0.0204 \\
\hline \multicolumn{2}{|c|}{261 Turbine_61 } & 0.48 & 1.0000 & 0.75 & -0.0301 \\
\hline \multicolumn{2}{|c|}{262 Turbine 62} & 0.48 & 1.0000 & 0.75 & -0.0383 \\
\hline \multicolumn{2}{|c|}{263 Turbine_63 } & 0.48 & 1.0000 & 0.75 & -0.0448 \\
\hline \multicolumn{2}{|c|}{264 Turbine 64} & 0.48 & 1.0000 & 0.75 & -0.0545 \\
\hline \multicolumn{2}{|c|}{265 Turbine 65} & 0.48 & 1.0000 & 0.75 & -0.0577 \\
\hline \multirow{3}{*}{\multicolumn{2}{|c|}{266 Turbine_66 }} & 0.48 & 1.0000 & 0.75 & -0.0594 \\
\hline & & & & 49.5 & -1.6994 Total Wind Generation \\
\hline & & & & 1.2024 & 9.0632 Total Losses \\
\hline & Mission_25 & 24.9 & 0.9865 & & \\
\hline 10 & Riser_A & 24.9 & 0.9949 & & \\
\hline 15 & Riser_B & 24.9 & 0.9930 & & \\
\hline 20 & Riser_C & 24.9 & 0.9890 & & \\
\hline 101 & Wind_01 & 24.9 & 1.0011 & & \\
\hline 102 & Wind_02 & 24.9 & 1.0010 & & \\
\hline 103 & Wind_03 & 24.9 & 1.0008 & & \\
\hline 104 & Wind_04 & 24.9 & 1.0005 & & \\
\hline 105 & Wind 05 & 24.9 & 1.0001 & & \\
\hline 106 & Wind_06 & 24.9 & 0.9997 & & \\
\hline 107 & Wind_07 & 24.9 & 0.9991 & & \\
\hline 108 & Wind_08 & 24.9 & 0.9985 & & \\
\hline 109 & Wind_09 & 24.9 & 0.9977 & & \\
\hline 110 & Wind_10 & 24.9 & 0.9969 & & \\
\hline 111 & Wind_11 & 24.9 & 0.9959 & & \\
\hline 112 & Wind_12 & 24.9 & 0.9960 & & \\
\hline 113 & Wind_13 & 24.9 & 0.9970 & & \\
\hline 114 & Wind_14 & 24.9 & 0.9980 & & \\
\hline 115 & Wind_15 & 24.9 & 0.9988 & & \\
\hline 116 & Wind_16 & 24.9 & 0.9996 & & \\
\hline 117 & Wind 17 & 24.9 & 1.0002 & & \\
\hline 118 & Wind_18 & 24.9 & 1.0007 & & \\
\hline 119 & Wind_19 & 24.9 & 1.0012 & & \\
\hline 120 & Wind_20 & 24.9 & 1.0016 & & \\
\hline 121 & Wind_21 & 24.9 & 1.0019 & & \\
\hline 122 & Wind_22 & 24.9 & 1.0020 & & \\
\hline 123 & Wind_23 & 24.9 & 1.0021 & & \\
\hline 124 & Wind_24 & 24.9 & 0.9958 & & \\
\hline 125 & Wind_25 & 24.9 & 0.9957 & & \\
\hline 126 & Wind_26 & 24.9 & 0.9955 & & \\
\hline 127 & Wind 27 & 24.9 & 0.9952 & & \\
\hline 128 & Wind_28 & 24.9 & 0.9948 & & \\
\hline 129 & Wind_29 & 24.9 & 0.9943 & & \\
\hline 130 & Wind_30 & 24.9 & 0.9937 & & \\
\hline 131 & Wind_31 & 24.9 & 0.9934 & & \\
\hline 132 & Wind_32 & 24.9 & 0.9938 & & \\
\hline 133 & Wind_33 & 24.9 & 0.9945 & & \\
\hline 134 & Wind_34 & 24.9 & 0.9952 & & \\
\hline
\end{tabular}


Table C3.1

Mission Wind Farm - 750 kW / 25 kV

WindPACT Turbine Design Scaling Studies

Power Flow Bus Report (100\% Generation, 49.5 MW)

\begin{tabular}{|c|c|c|c|c|}
\hline \multicolumn{2}{|r|}{ Bus } & \multirow{2}{*}{$\frac{\text { Base }}{\mathrm{kV}}$} & \multirow{2}{*}{$\begin{array}{c}\text { Voltage } \\
\text { P.U. }\end{array}$} & Generation \\
\hline Number & Name & & & \begin{tabular}{l|l}
$P(M W)$ & $Q(M V A r)$ \\
\end{tabular} \\
\hline 135 & Wind_35 & 24.9 & 0.9958 & \\
\hline 136 & Wind_36 & 24.9 & 0.9964 & \\
\hline 137 & Wind_37 & 24.9 & 0.9975 & \\
\hline $13 \xi$ & Wind_38 & 24.9 & 0.9986 & \\
\hline 135 & Wind_39 & 24.9 & 0.9995 & \\
\hline 140 & Wind_40 & 24.9 & 1.0003 & \\
\hline 14 & Wind_41 & 24.9 & 1.0010 & \\
\hline 14 & Wind_42 & 24.9 & 1.0017 & \\
\hline 143 & Wind_43 & 24.9 & 1.0022 & \\
\hline 14 & Wind_44 & 24.9 & 1.0027 & \\
\hline 145 & Wind_45 & 24.9 & 1.0030 & \\
\hline 146 & Wind_46 & 24.9 & 1.0033 & \\
\hline 147 & Wind_47 & 24.9 & 1.0035 & \\
\hline $14 \varepsilon$ & Wind_48 & 24.9 & 1.0036 & \\
\hline $14 \varsigma$ & Wind_49 & 24.9 & 0.9893 & \\
\hline 150 & Wind_50 & 24.9 & 0.9892 & \\
\hline 15 & Wind_51 & 24.9 & 0.9891 & \\
\hline 15 & Wind_52 & 24.9 & 0.9897 & \\
\hline 15 & Wind_53 & 24.9 & 0.9904 & \\
\hline 15 & Wind_54 & 24.9 & 0.9910 & \\
\hline 155 & Wind_55 & 24.9 & 0.9921 & \\
\hline 156 & Wind_56 & 24.9 & 0.9932 & \\
\hline 157 & Wind_57 & 24.9 & 0.9951 & \\
\hline $15 \varepsilon$ & Wind_58 & 24.9 & 0.9959 & \\
\hline $15 \varsigma$ & Wind_59 & 24.9 & 0.9967 & \\
\hline 16 & Wind_60 & 24.9 & 0.9973 & \\
\hline 16 & Wind_61 & 24.9 & 0.9979 & \\
\hline 16 & Wind_62 & 24.9 & 0.9984 & \\
\hline 16 & Wind_63 & 24.9 & 0.9987 & \\
\hline 16 & Wind_64 & 24.9 & 0.9993 & \\
\hline 165 & Wind_65 & 24.9 & 0.9995 & \\
\hline 166 & Wind_66 & 24.9 & 0.9996 & \\
\hline
\end{tabular}

C3 - 3 
Table C3.2

Mission Wind Farm - 750 kW / 25 kV

WindPACT Turbine Design Scaling Studies

Power Flow Bus Report (100\% Generation, 49.5 MW)

\begin{tabular}{|c|c|c|c|c|c|c|c|c|}
\hline From Bus & To Bus & Ckt & MW & MVar & MVA & AMPS & Norm & \%Norm \\
\hline 136 Wind_36 & 137 Wind_37 & 1 & -8.919 & 1.448 & 9.036 & 210 & 9 & 100.4 \\
\hline 112 Wind_12 & 10 Riser_A & 1 & 8.919 & -1.143 & 8.992 & 209 & 9 & 99.9 \\
\hline 154 Wind_54 & 155 Wind_55 & 1 & -8.912 & 0.511 & 8.927 & 209 & 9 & 99.2 \\
\hline 1 Mission_115 & 2 Mission_25 & 1 & -48.298 & 10.763 & 49.482 & 1147 & 50 & 99.0 \\
\hline 20 Riser_C & 2 Mission_25 & 1 & 48.797 & -3.710 & 48.938 & 1147 & 50 & 97.9 \\
\hline 132 Wind_32 & 15 Riser_B & 1 & 12.609 & -1.514 & 12.700 & 296 & 13 & 97.7 \\
\hline 137 Wind_37 & 138 Wind_38 & 1 & -8.181 & 1.393 & 8.299 & 193 & 9 & 92.2 \\
\hline 132 Wind 32 & 133 Wind 33 & 1 & -11.872 & 1.523 & 11.969 & 279 & 13 & 92.1 \\
\hline 112 Wind_12 & 113 Wind_13 & 1 & -8.182 & 1.115 & 8.257 & 192 & 9 & 91.7 \\
\hline 111 Wind_11 & 10 Riser_A & 1 & 8.182 & -0.902 & 8.232 & 192 & 9 & 91.5 \\
\hline 155 Wind_55 & 156 Wind_56 & 1 & -8.175 & 0.550 & 8.193 & 191 & 9 & 91.0 \\
\hline 133 Wind_33 & 134 Wind_34 & 1 & -11.134 & 1.521 & 11.237 & 262 & 13 & 86.4 \\
\hline 152 Wind_52 & 20 Riser_C & 1 & 11.127 & -0.300 & 11.131 & 261 & 13 & 85.6 \\
\hline 138 Wind_38 & 139 Wind_39 & 1 & -7.442 & 1.322 & 7.559 & 175 & 9 & 84.0 \\
\hline 113 Wind_13 & 114 Wind_14 & 1 & -7.443 & 1.069 & 7.519 & 175 & 9 & 83.5 \\
\hline 110 Wind_10 & 111 Wind_11 & 1 & 7.443 & -0.876 & 7.494 & 174 & 9 & 83.3 \\
\hline 156 Wind_56 & 157 Wind_57 & 1 & -7.443 & 0.575 & 7.465 & 174 & 9 & 82.9 \\
\hline 134 Wind_34 & 135 Wind_35 & 1 & -10.395 & 1.507 & 10.503 & 245 & 13 & 80.8 \\
\hline 152 Wind_52 & 153 Wind_53 & 1 & -10.388 & 0.382 & 10.395 & 243 & 13 & 80.0 \\
\hline 148 Wind_48 & 248 Turbine_48 & 1 & -0.746 & 0.162 & 0.764 & 915 & 1 & 76.4 \\
\hline 147 Wind_47 & 247 Turbine_47 & 1 & -0.746 & 0.161 & 0.763 & 915 & 1 & 76.3 \\
\hline 146 Wind_46 & 246 Turbine_46 & 1 & -0.746 & 0.157 & 0.763 & 914 & 1 & 76.3 \\
\hline 145 Wind_45 & 245 Turbine_45 & 1 & -0.746 & 0.153 & 0.762 & 913 & 1 & 76.2 \\
\hline 144 Wind_44 & 244 Turbine_44 & 1 & -0.746 & 0.146 & 0.761 & 912 & 1 & 76.1 \\
\hline 151 Wind_51 & 251 Turbine_51 & 1 & -0.750 & -0.122 & 0.760 & 914 & 1 & 76.0 \\
\hline 150 Wind_50 & 250 Turbine_50 & 1 & -0.750 & -0.120 & 0.760 & 914 & 1 & 76.0 \\
\hline 149 Wind_49 & 249 Turbine_49 & 1 & -0.750 & -0.119 & 0.759 & 913 & 1 & 75.9 \\
\hline 143 Wind_43 & 243 Turbine_43 & 1 & -0.746 & 0.138 & 0.759 & 911 & 1 & 75.9 \\
\hline 123 Wind_23 & 223 Turbine_23 & 1 & -0.746 & 0.137 & 0.759 & 911 & 1 & 75.9 \\
\hline 122 Wind_22 & 222 Turbine_22 & 1 & -0.746 & 0.135 & 0.758 & 910 & 1 & 75.8 \\
\hline 152 Wind_52 & 252 Turbine_52 & 1 & -0.750 & -0.112 & 0.758 & 912 & 1 & 75.8 \\
\hline 121 Wind_21 & 221 Turbine_21 & 1 & -0.746 & 0.132 & 0.758 & 910 & 1 & 75.8 \\
\hline 139 Wind_39 & 140 Wind_40 & 1 & -6.702 & 1.235 & 6.815 & 158 & 9 & 75.7 \\
\hline 142 Wind_42 & 242 Turbine_42 & 1 & -0.746 & 0.129 & 0.757 & 909 & 1 & 75.7 \\
\hline 120 Wind_20 & 220 Turbine_20 & 1 & -0.746 & 0.127 & 0.757 & 909 & 1 & 75.7 \\
\hline 153 Wind_53 & 253 Turbine_53 & 1 & -0.750 & -0.100 & 0.757 & 910 & 1 & 75.7 \\
\hline 119 Wind_19 & 219 Turbine_19 & 1 & -0.746 & 0.121 & 0.756 & 908 & 1 & 75.6 \\
\hline 101 Wind_01 & 201 Turbine_01 & 1 & -0.746 & 0.118 & 0.756 & 908 & 1 & 75.6 \\
\hline 141 Wind_41 & 241 Turbine_41 & 1 & -0.746 & 0.117 & 0.756 & 908 & 1 & 75.6 \\
\hline 102 Wind_02 & 202 Turbine_02 & 1 & -0.746 & 0.116 & 0.755 & 908 & 1 & 75.5 \\
\hline 154 Wind_54 & 254 Turbine_54 & 1 & -0.750 & -0.089 & 0.755 & 909 & 1 & 75.5 \\
\hline 103 Wind_03 & 203 Turbine_03 & 1 & -0.746 & 0.113 & 0.755 & 907 & 1 & 75.5 \\
\hline 118 Wind_18 & 218 Turbine_18 & 1 & -0.746 & 0.113 & 0.755 & 907 & 1 & 75.5 \\
\hline 104 Wind_04 & 204 Turbine_04 & 1 & -0.746 & 0.108 & 0.754 & 907 & 1 & 75.4 \\
\hline 140 Wind_40 & 240 Turbine_40 & 1 & -0.746 & 0.105 & 0.754 & 906 & 1 & 75.4 \\
\hline 114 Wind_14 & 115 Wind_15 & 1 & -6.702 & 1.008 & 6.778 & 157 & 9 & 75.3 \\
\hline 117 Wind_17 & 217 Turbine_17 & 1 & -0.746 & 0.103 & 0.753 & 906 & 1 & 75.3 \\
\hline 105 Wind_05 & 205 Turbine_05 & 1 & -0.746 & 0.102 & 0.753 & 906 & 1 & 75.3 \\
\hline 155 Wind_55 & 255 Turbine_55 & 1 & -0.750 & -0.070 & 0.753 & 906 & 1 & 75.3 \\
\hline 106 Wind_06 & 206 Turbine_06 & 1 & -0.750 & 0.061 & 0.752 & 905 & 1 & 75.2 \\
\hline 166 Wind_66 & 266 Turbine_66 & 1 & -0.750 & 0.059 & 0.752 & 905 & 1 & 75.2 \\
\hline 116 Wind_16 & 216 Turbine_16 & 1 & -0.750 & 0.059 & 0.752 & 905 & 1 & 75.2 \\
\hline 165 Wind_65 & 265 Turbine_65 & 1 & -0.750 & 0.058 & 0.752 & 905 & 1 & 75.2 \\
\hline 139 Wind_39 & 239 Turbine_39 & 1 & -0.750 & 0.058 & 0.752 & 905 & 1 & 75.2 \\
\hline 164 Wind_64 & 264 Turbine_64 & 1 & -0.750 & 0.054 & 0.752 & 904 & 1 & 75.2 \\
\hline
\end{tabular}


Table C3.2

Mission Wind Farm - 750 kW / 25 kV

WindPACT Turbine Design Scaling Studies

Power Flow Bus Report (100\% Generation, 49.5 MW)

\begin{tabular}{|c|c|c|c|c|c|c|c|c|}
\hline From Bus & To Bus & Ckt & MW & MVar & MVA & AMPS & Norm & $\%$ Norm \\
\hline 156 Wind_56 & 256 Turbine_56 & 1 & -0.750 & -0.052 & 0.752 & 904 & 1 & 75.2 \\
\hline 107 Wind_07 & 207 Turbine_07 & 1 & -0.750 & 0.051 & 0.752 & 904 & 1 & 75.2 \\
\hline 131 Wind_31 & 231 Turbine_31 & 1 & -0.750 & -0.048 & 0.752 & 904 & 1 & 75.2 \\
\hline 135 Wind_35 & 136 Wind_36 & 1 & -9.655 & 1.483 & 9.768 & 227 & 13 & 75.1 \\
\hline 115 Wind_15 & 215 Turbine_15 & 1 & -0.750 & 0.046 & 0.751 & 904 & 1 & 75.1 \\
\hline 163 Wind_63 & 263 Turbine_63 & 1 & -0.750 & 0.045 & 0.751 & 904 & 1 & 75.1 \\
\hline 138 Wind_38 & 238 Turbine_38 & 1 & -0.750 & 0.042 & 0.751 & 904 & 1 & 75.1 \\
\hline 130 Wind_30 & 230 Turbine_30 & 1 & -0.750 & -0.042 & 0.751 & 904 & 1 & 75.1 \\
\hline 132 Wind_32 & 232 Turbine_32 & 1 & -0.750 & -0.041 & 0.751 & 903 & 1 & 75.1 \\
\hline 108 Wind_08 & 208 Turbine_08 & 1 & -0.750 & 0.040 & 0.751 & 903 & 1 & 75.1 \\
\hline 162 Wind_62 & 262 Turbine_62 & 1 & -0.750 & 0.038 & 0.751 & 903 & 1 & 75.1 \\
\hline 114 Wind_14 & 214 Turbine_14 & 1 & -0.750 & 0.032 & 0.751 & 903 & 1 & 75.1 \\
\hline 129 Wind_29 & 229 Turbine_29 & 1 & -0.750 & -0.032 & 0.751 & 903 & 1 & 75.1 \\
\hline 161 Wind_61 & 261 Turbine_61 & 1 & -0.750 & 0.030 & 0.751 & 903 & 1 & 75.1 \\
\hline 133 Wind_33 & 233 Turbine_33 & 1 & -0.750 & -0.028 & 0.751 & 903 & 1 & 75.1 \\
\hline 109 Wind_09 & 110 Wind_10 & 1 & 6.702 & -0.834 & 6.754 & 157 & 9 & 75.0 \\
\hline 109 Wind_09 & 209 Turbine_09 & 1 & -0.750 & 0.027 & 0.750 & 903 & 1 & 75.0 \\
\hline 137 Wind_37 & 237 Turbine_37 & 1 & -0.750 & 0.024 & 0.750 & 903 & 1 & 75.0 \\
\hline 128 Wind_28 & 228 Turbine_28 & 1 & -0.750 & -0.023 & 0.750 & 903 & 1 & 75.0 \\
\hline 160 Wind_60 & 260 Turbine_60 & 1 & -0.750 & 0.020 & 0.750 & 902 & 1 & 75.0 \\
\hline 157 Wind_57 & 257 Turbine_57 & 1 & -0.750 & -0.019 & 0.750 & 902 & 1 & 75.0 \\
\hline 127 Wind_27 & 227 Turbine_27 & 1 & -0.750 & -0.017 & 0.750 & 902 & 1 & 75.0 \\
\hline 134 Wind_34 & 234 Turbine_34 & 1 & -0.750 & -0.016 & 0.750 & 902 & 1 & 75.0 \\
\hline 113 Wind_13 & 213 Turbine_13 & 1 & -0.750 & 0.016 & 0.750 & 902 & 1 & 75.0 \\
\hline 110 Wind_10 & 210 Turbine_10 & 1 & -0.750 & 0.013 & 0.750 & 902 & 1 & 75.0 \\
\hline 126 Wind_26 & 226 Turbine_26 & 1 & -0.750 & -0.012 & 0.750 & 902 & 1 & 75.0 \\
\hline 159 Wind_59 & 259 Turbine_59 & 1 & -0.750 & 0.009 & 0.750 & 902 & 1 & 75.0 \\
\hline 125 Wind_25 & 225 Turbine_25 & 1 & -0.750 & -0.008 & 0.750 & 902 & 1 & 75.0 \\
\hline 124 Wind_24 & 224 Turbine_24 & 1 & -0.750 & -0.007 & 0.750 & 902 & 1 & 75.0 \\
\hline 135 Wind_35 & 235 Turbine_35 & 1 & -0.750 & -0.005 & 0.750 & 902 & 1 & 75.0 \\
\hline 136 Wind_36 & 236 Turbine_36 & 1 & -0.750 & 0.005 & 0.750 & 902 & 1 & 75.0 \\
\hline 158 Wind_58 & 258 Turbine_58 & 1 & -0.750 & -0.004 & 0.750 & 902 & 1 & 75.0 \\
\hline 111 Wind_11 & 211 Turbine_11 & 1 & -0.750 & -0.004 & 0.750 & 902 & 1 & 75.0 \\
\hline 112 Wind_12 & 212 Turbine_12 & 1 & -0.750 & -0.002 & 0.750 & 902 & 1 & 75.0 \\
\hline 157 Wind_57 & 158 Wind_58 & 1 & -6.702 & 0.564 & 6.726 & 157 & 9 & 74.7 \\
\hline 153 Wind_53 & 154 Wind_54 & 1 & -9.648 & 0.452 & 9.659 & 226 & 13 & 74.3 \\
\hline 15 Riser_B & 20 Riser_C & 1 & 35.603 & -3.574 & 35.782 & 836 & 50 & 71.6 \\
\hline 140 Wind_40 & 141 Wind_41 & 1 & -5.960 & 1.134 & 6.067 & 141 & 9 & 67.4 \\
\hline 115 Wind_15 & 116 Wind_16 & 1 & -5.961 & 0.933 & 6.033 & 140 & 9 & 67.0 \\
\hline 108 Wind_08 & 109 Wind_09 & 1 & 5.961 & -0.778 & 6.011 & 140 & 9 & 66.8 \\
\hline 158 Wind_58 & 159 Wind_59 & 1 & -5.960 & 0.540 & 5.985 & 139 & 9 & 66.5 \\
\hline 141 Wind_41 & 142 Wind_42 & 1 & -5.217 & 1.020 & 5.316 & 123 & 9 & 59.1 \\
\hline 116 Wind_16 & 117 Wind_17 & 1 & -5.218 & 0.845 & 5.286 & 123 & 9 & 58.7 \\
\hline 107 Wind_07 & 108 Wind_08 & 1 & 5.218 & -0.709 & 5.266 & 122 & 9 & 58.5 \\
\hline 159 Wind_59 & 160 Wind_60 & 1 & -5.217 & 0.502 & 5.242 & 122 & 9 & 58.2 \\
\hline 142 Wind_42 & 143 Wind_43 & 1 & -4.474 & 0.895 & 4.562 & 106 & 9 & 50.7 \\
\hline 117 Wind_17 & 118 Wind_18 & 1 & -4.474 & 0.746 & 4.536 & 105 & 9 & 50.4 \\
\hline 106 Wind_06 & 107 Wind_07 & 1 & 4.474 & -0.629 & 4.518 & 105 & 9 & 50.2 \\
\hline 160 Wind_60 & 161 Wind_61 & 1 & -4.474 & 0.453 & 4.497 & 104 & 9 & 50.0 \\
\hline 129 Wind_29 & 130 Wind_30 & 1 & 4.474 & -0.074 & 4.475 & 104 & 9 & 49.7 \\
\hline 131 Wind_31 & 15 Riser_B & 1 & 5.963 & -0.041 & 5.963 & 139 & 13 & 45.9 \\
\hline 143 Wind_43 & 144 Wind_44 & 1 & -3.729 & 0.761 & 3.806 & 88 & 9 & 42.3 \\
\hline 118 Wind_18 & 119 Wind_19 & 1 & -3.730 & 0.638 & 3.784 & 88 & 9 & 42.0 \\
\hline 105 Wind_05 & 106 Wind_06 & 1 & 3.730 & -0.539 & 3.768 & 87 & 9 & 41.9 \\
\hline 161 Wind_61 & 162 Wind_62 & 1 & -3.729 & 0.395 & 3.750 & 87 & 9 & 41.7 \\
\hline
\end{tabular}


Table C3.2

Mission Wind Farm - 750 kW / 25 kV

WindPACT Turbine Design Scaling Studies

Power Flow Bus Report (100\% Generation, 49.5 MW)

\begin{tabular}{|c|c|c|c|c|c|c|c|c|}
\hline From Bus & To Bus & Ckt & MW & MVar & MVA & AMPS & Norm & \%Norm \\
\hline 128 Wind_28 & 129 Wind_29 & 1 & 3.730 & -0.077 & 3.731 & 87 & 9 & 41.5 \\
\hline 130 Wind_30 & 131 Wind_31 & 1 & 5.218 & -0.061 & 5.219 & 122 & 13 & 40.1 \\
\hline 10 Riser_A & 15 Riser_B & 1 & 17.082 & -2.040 & 17.204 & 401 & 50 & 34.4 \\
\hline 144 Wind_44 & 145 Wind_45 & 1 & -2.984 & 0.619 & 3.048 & 70 & 9 & 33.9 \\
\hline 119 Wind 19 & 120 Wind 20 & 1 & -2.984 & 0.522 & 3.030 & 70 & 9 & 33.7 \\
\hline 104 Wind_04 & 105 Wind_05 & 1 & 2.984 & -0.442 & 3.017 & 70 & 9 & 33.5 \\
\hline 162 Wind 62 & 163 Wind 63 & 1 & -2.984 & 0.328 & 3.002 & 70 & 9 & 33.4 \\
\hline 127 Wind_27 & 128 Wind_28 & 1 & 2.985 & -0.072 & 2.985 & 70 & 9 & 33.2 \\
\hline 145 Wind_45 & 146 Wind_46 & 1 & -2.239 & 0.471 & 2.288 & 53 & 9 & 25.4 \\
\hline 120 Wind_20 & 121 Wind_21 & 1 & -2.239 & 0.399 & 2.274 & 53 & 9 & 25.3 \\
\hline 103 Wind_03 & 104 Wind_04 & 1 & 2.239 & -0.338 & 2.264 & 52 & 9 & 25.2 \\
\hline 163 Wind_63 & 164 Wind_64 & 1 & -2.239 & 0.260 & 2.254 & 52 & 9 & 25.0 \\
\hline 126 Wind_26 & 127 Wind_27 & 1 & 2.239 & -0.061 & 2.240 & 52 & 9 & 24.9 \\
\hline 151 Wind_51 & 20 Riser_C & 1 & 2.238 & 0.277 & 2.256 & 53 & 13 & 17.4 \\
\hline 146 Wind_46 & 147 Wind_47 & 1 & -1.493 & 0.318 & 1.526 & 35 & 9 & 17.0 \\
\hline 121 Wind_21 & 122 Wind_22 & 1 & -1.493 & 0.272 & 1.517 & 35 & 9 & 16.9 \\
\hline 102 Wind_02 & 103 Wind_03 & 1 & 1.493 & -0.230 & 1.510 & 35 & 9 & 16.8 \\
\hline 164 Wind_64 & 165 Wind_65 & 1 & -1.493 & 0.178 & 1.503 & 35 & 9 & 16.7 \\
\hline 125 Wind_25 & 126 Wind_26 & 1 & 1.493 & -0.045 & 1.493 & 35 & 9 & 16.6 \\
\hline 150 Wind_50 & 151 Wind_51 & 1 & 1.492 & 0.184 & 1.504 & 35 & 13 & 11.6 \\
\hline 147 Wind_47 & 148 Wind_48 & 1 & -0.746 & 0.162 & 0.764 & 18 & 9 & 8.5 \\
\hline 122 Wind_22 & 123 Wind_23 & 1 & -0.746 & 0.137 & 0.759 & 18 & 9 & 8.4 \\
\hline 101 Wind_01 & 102 Wind_02 & 1 & 0.746 & -0.118 & 0.756 & 18 & 9 & 8.4 \\
\hline 165 Wind_65 & 166 Wind_66 & 1 & -0.746 & 0.092 & 0.752 & 17 & 9 & 8.4 \\
\hline 124 Wind_24 & 125 Wind_25 & 1 & 0.746 & -0.026 & 0.747 & 17 & 9 & 8.3 \\
\hline 149 Wind_49 & 150 Wind_50 & 1 & 0.746 & 0.091 & 0.752 & 18 & 13 & 5.8 \\
\hline
\end{tabular}


Table C4.1

Mission Wind Farm - 2500 kW / 25 kV

WindPACT Turbine Design Scaling Studies

Power Flow Bus Report (100\% Generation, 50 MW)

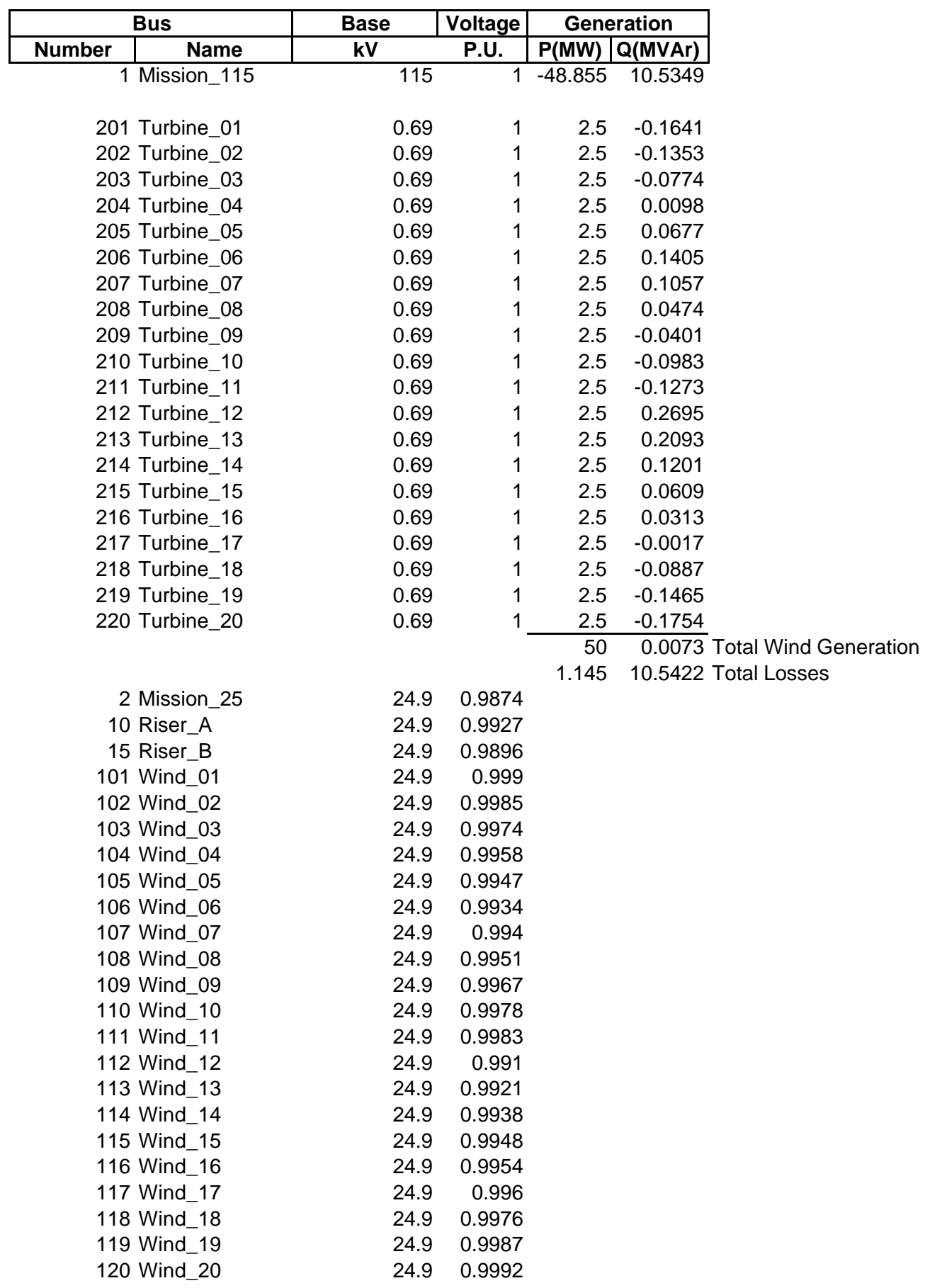


Table C4.2

Mission Wind Farm - 2500 kW / 25 kV

WindPACT Turbine Design Scaling Studies

Power Flow Bus Report (100\% Generation, 50 MW)

\begin{tabular}{|c|c|c|c|c|c|c|c|c|}
\hline From Bus & To Bus & Ckt & MW & MVar & MVA & AMPS & Norm & \%Norm \\
\hline 1 Mission_115 & 2 Mission_25 & 1 & -48.856 & 10.539 & 49.980 & 1159 & 50 & 100.0 \\
\hline 15 Riser_B & 2 Mission_25 & 1 & 49.391 & -2.726 & 49.466 & 1159 & 50 & 98.9 \\
\hline 112 Wind_12 & 15 Riser_B & 1 & 12.422 & 0.234 & 12.424 & 291 & 13 & 95.6 \\
\hline 105 Wind_05 & 106 Wind_06 & 1 & 12.113 & -1.027 & 12.156 & 283 & 13 & 93.5 \\
\hline 107 Wind_07 & 10 Riser_A & 1 & 12.047 & -0.891 & 12.080 & 282 & 13 & 92.9 \\
\hline 113 Wind 13 & 114 Wind_14 & 1 & -7.704 & 0.032 & 7.704 & 180 & 9 & 85.6 \\
\hline 112 Wind_12 & 212 Turbine_12 & 1 & -2.500 & -0.270 & 2.514 & 2104 & 3 & 83.8 \\
\hline 113 Wind_13 & 213 Turbine_13 & 1 & -2.500 & -0.210 & 2.509 & 2099 & 3 & 83.6 \\
\hline 120 Wind_20 & 220 Turbine_20 & 1 & -2.499 & 0.176 & 2.506 & 2097 & 3 & 83.5 \\
\hline 101 Wind_01 & 201 Turbine_01 & 1 & -2.500 & 0.165 & 2.506 & 2096 & 3 & 83.5 \\
\hline 119 Wind_19 & 219 Turbine_19 & 1 & -2.500 & 0.149 & 2.505 & 2096 & 3 & 83.5 \\
\hline 106 Wind_06 & 206 Turbine_06 & 1 & -2.500 & -0.139 & 2.504 & 2095 & 3 & 83.5 \\
\hline 102 Wind_02 & 202 Turbine_02 & 1 & -2.500 & 0.138 & 2.504 & 2095 & 3 & 83.5 \\
\hline 114 Wind_14 & 214 Turbine_14 & 1 & -2.500 & -0.117 & 2.503 & 2095 & 3 & 83.4 \\
\hline 111 Wind_11 & 211 Turbine_11 & 1 & -2.500 & 0.127 & 2.503 & 2094 & 3 & 83.4 \\
\hline 110 Wind_10 & 210 Turbine_10 & 1 & -2.501 & 0.100 & 2.503 & 2094 & 3 & 83.4 \\
\hline 107 Wind 07 & 207 Turbine 07 & 1 & -2.500 & -0.107 & 2.502 & 2094 & 3 & 83.4 \\
\hline 118 Wind_18 & 218 Turbine_18 & 1 & -2.500 & 0.089 & 2.502 & 2093 & 3 & 83.4 \\
\hline 103 Wind_03 & 203 Turbine_03 & 1 & -2.500 & 0.078 & 2.501 & 2093 & 3 & 83.4 \\
\hline 105 Wind_05 & 205 Turbine_05 & 1 & -2.500 & -0.069 & 2.501 & 2093 & 3 & 83.4 \\
\hline 108 Wind_08 & 208 Turbine_08 & 1 & -2.501 & -0.047 & 2.501 & 2093 & 3 & 83.4 \\
\hline 115 Wind_15 & 215 Turbine_15 & 1 & -2.500 & -0.063 & 2.501 & 2092 & 3 & 83.4 \\
\hline 117 Wind_17 & 217 Turbine_17 & 1 & -2.500 & 0.002 & 2.500 & 2092 & 3 & 83.3 \\
\hline 104 Wind_04 & 204 Turbine_04 & 1 & -2.500 & -0.009 & 2.500 & 2092 & 3 & 83.3 \\
\hline 109 Wind_09 & 209 Turbine_09 & 1 & -2.500 & 0.040 & 2.500 & 2092 & 3 & 83.3 \\
\hline 116 Wind_16 & 216 Turbine_16 & 1 & -2.500 & -0.031 & 2.500 & 2092 & 3 & 83.3 \\
\hline 117 Wind_17 & 118 Wind_18 & 1 & -7.449 & 0.740 & 7.486 & 174 & 9 & 83.2 \\
\hline 103 Wind_03 & 104 Wind_04 & 1 & 7.429 & -0.665 & 7.459 & 173 & 9 & 82.9 \\
\hline 108 Wind_08 & 109 Wind_09 & 1 & -7.424 & 0.663 & 7.453 & 173 & 9 & 82.8 \\
\hline 104 Wind_04 & 105 Wind_05 & 1 & 10.215 & -0.765 & 10.244 & 239 & 13 & 78.8 \\
\hline 107 Wind_07 & 108 Wind_08 & 1 & -10.094 & 0.499 & 10.106 & 235 & 13 & 77.7 \\
\hline 117 Wind 17 & 15 Riser B & 1 & 9.941 & -0.827 & 9.975 & 232 & 13 & 76.7 \\
\hline 112 Wind_12 & 113 Wind_13 & 1 & -9.889 & 0.093 & 9.890 & 231 & 13 & 76.1 \\
\hline 106 Wind_06 & 10 Riser_A & 1 & 14.735 & -0.892 & 14.762 & 345 & 21 & 70.3 \\
\hline 118 Wind_18 & 119 Wind_19 & 1 & -5.127 & 0.513 & 5.153 & 120 & 9 & 57.3 \\
\hline 102 Wind_02 & 103 Wind_03 & 1 & 5.126 & -0.513 & 5.152 & 120 & 9 & 57.2 \\
\hline 109 Wind_09 & 110 Wind_10 & 1 & -5.087 & 0.362 & 5.099 & 118 & 9 & 56.7 \\
\hline 10 Riser_A & 15 Riser_B & 1 & 27.251 & -1.422 & 27.288 & 637 & 50 & 54.6 \\
\hline 114 Wind_14 & 115 Wind_15 & 1 & -4.588 & 0.237 & 4.594 & 107 & 9 & 51.0 \\
\hline 115 Wind_15 & 116 Wind_16 & 1 & -2.733 & 0.055 & 2.734 & 64 & 9 & 30.4 \\
\hline 119 Wind_19 & 120 Wind_20 & 1 & -2.368 & 0.386 & 2.399 & 56 & 9 & 26.7 \\
\hline 101 Wind_01 & 102 Wind_02 & 1 & 2.349 & -0.311 & 2.370 & 55 & 9 & 26.3 \\
\hline 110 Wind_10 & 111 Wind_11 & 1 & -2.347 & 0.310 & 2.368 & 55 & 9 & 26.3 \\
\hline
\end{tabular}


Table C5.1

Mission Wind Farm - 2500 kW / 35 kV

WindPACT Turbine Design Scaling Studies

Power Flow Bus Report (100\% Generation, 50 MW)

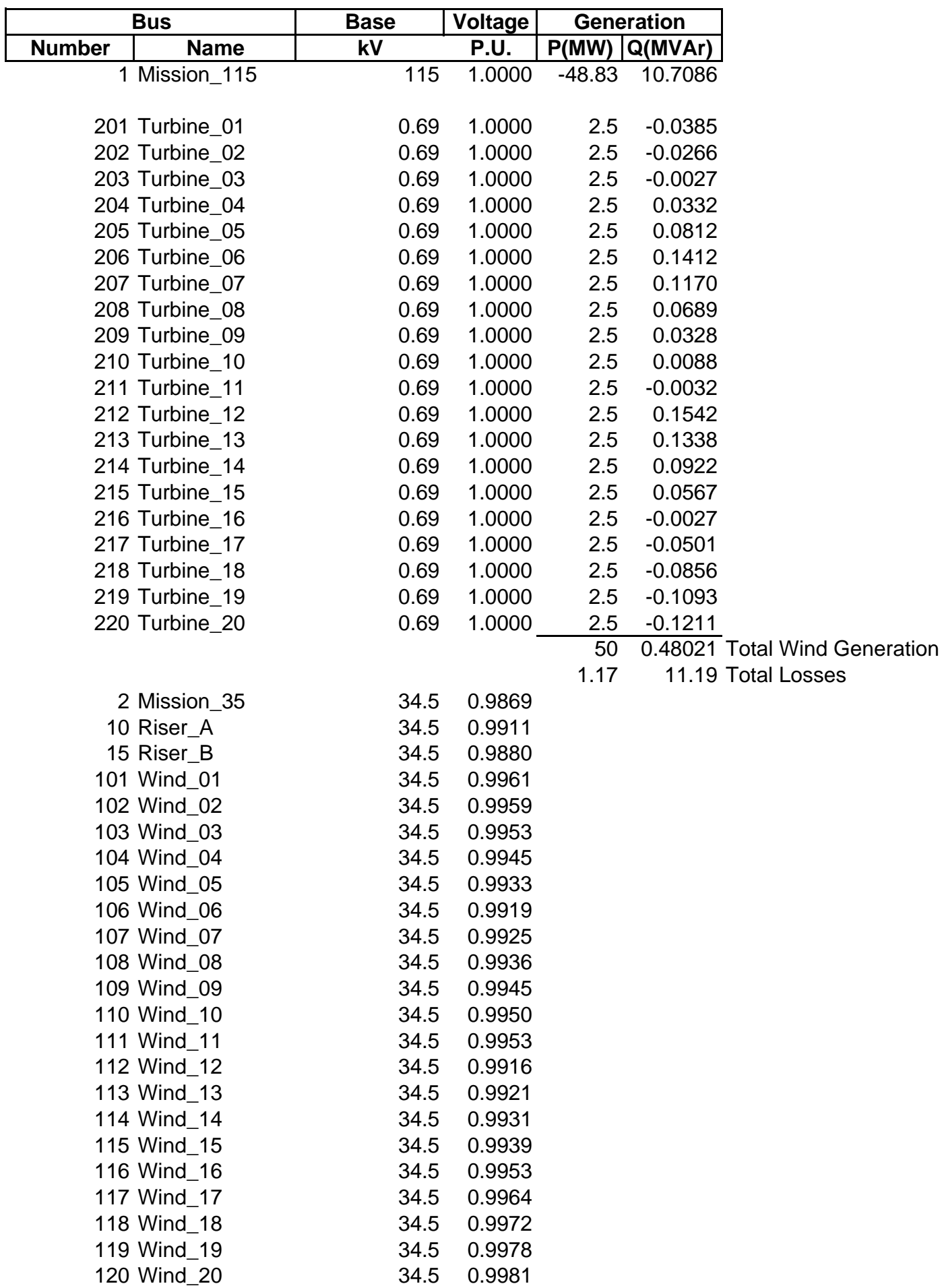


Table C5.2

Mission Wind Farm - 2500 kW / 35 kV

WindPACT Turbine Design Scaling Studies

Power Flow Bus Report (100\% Generation, 50 MW)

\begin{tabular}{|c|c|c|c|c|c|c|c|c|}
\hline From Bus & To Bus & Ckt & MW & MVar & MVA & AMPS & Norm & \%Norm \\
\hline 1 Mission_115 & 2 Mission_35 & 1 & -48.828 & 10.713 & 49.990 & 837 & 50 & 100.0 \\
\hline 115 Wind_15 & 116 Wind_16 & 1 & -12.482 & 1.142 & 12.534 & 211 & 13 & 96.4 \\
\hline 105 Wind_05 & 106 Wind_06 & 1 & 12.345 & -0.709 & 12.365 & 208 & 13 & 95.1 \\
\hline 107 Wind_07 & 10 Riser_A & 1 & 12.297 & -0.565 & 12.310 & 208 & 13 & 94.7 \\
\hline 113 Wind_13 & 114 Wind_14 & 1 & -17.728 & 1.134 & 17.764 & 299 & 19 & 93.5 \\
\hline 10 Riser_A & 15 Riser_B & 1 & 49.501 & -2.201 & 49.550 & 837 & 54 & 91.8 \\
\hline 15 Riser_B & 2 Mission_35 & 1 & 49.311 & -3.198 & 49.415 & 837 & 54 & 91.5 \\
\hline 112 Wind_12 & 212 Turbine_12 & 1 & -2.501 & -0.155 & 2.505 & 2096 & 3 & 83.5 \\
\hline 106 Wind_06 & 206 Turbine_06 & 1 & -2.500 & -0.142 & 2.504 & 2095 & 3 & 83.5 \\
\hline 113 Wind_13 & 213 Turbine_13 & 1 & -2.500 & -0.133 & 2.504 & 2095 & 3 & 83.5 \\
\hline 120 Wind_20 & 220 Turbine_20 & 1 & -2.500 & 0.122 & 2.503 & 2094 & 3 & 83.4 \\
\hline 119 Wind_19 & 219 Turbine_19 & 1 & -2.500 & 0.110 & 2.502 & 2094 & 3 & 83.4 \\
\hline 107 Wind_07 & 207 Turbine_07 & 1 & -2.499 & -0.116 & 2.502 & 2094 & 3 & 83.4 \\
\hline 118 Wind_18 & 218 Turbine_18 & 1 & -2.500 & 0.084 & 2.501 & 2093 & 3 & 83.4 \\
\hline 115 Wind_15 & 215 Turbine_15 & 1 & -2.501 & -0.057 & 2.501 & 2093 & 3 & 83.4 \\
\hline 114 Wind_14 & 214 Turbine_14 & 1 & -2.499 & -0.091 & 2.501 & 2093 & 3 & 83.4 \\
\hline 105 Wind_05 & 205 Turbine_05 & 1 & -2.500 & -0.082 & 2.501 & 2093 & 3 & 83.4 \\
\hline 108 Wind_08 & 208 Turbine_08 & 1 & -2.500 & -0.070 & 2.501 & 2092 & 3 & 83.4 \\
\hline 111 Wind_11 & 211 Turbine_11 & 1 & -2.500 & 0.003 & 2.500 & 2092 & 3 & 83.3 \\
\hline 101 Wind_01 & 201 Turbine_01 & 1 & -2.500 & 0.037 & 2.500 & 2092 & 3 & 83.3 \\
\hline 110 Wind_10 & 210 Turbine_10 & 1 & -2.500 & -0.010 & 2.500 & 2092 & 3 & 83.3 \\
\hline 109 Wind_09 & 209 Turbine_09 & 1 & -2.500 & -0.031 & 2.500 & 2092 & 3 & 83.3 \\
\hline 104 Wind_04 & 204 Turbine_04 & 1 & -2.500 & -0.031 & 2.500 & 2092 & 3 & 83.3 \\
\hline 102 Wind_02 & 202 Turbine_02 & 1 & -2.500 & 0.029 & 2.500 & 2092 & 3 & 83.3 \\
\hline 117 Wind_17 & 217 Turbine_17 & 1 & -2.499 & 0.050 & 2.500 & 2092 & 3 & 83.3 \\
\hline 103 Wind_03 & 203 Turbine_03 & 1 & -2.500 & 0.003 & 2.500 & 2092 & 3 & 83.3 \\
\hline 116 Wind_16 & 216 Turbine_16 & 1 & -2.500 & 0.003 & 2.500 & 2092 & 3 & 83.3 \\
\hline 104 Wind_04 & 105 Wind_05 & 1 & 10.548 & -0.432 & 10.557 & 178 & 13 & 81.2 \\
\hline 116 Wind_16 & 117 Wind_17 & 1 & -9.827 & 0.934 & 9.871 & 166 & 13 & 75.9 \\
\hline 114 Wind_14 & 115 Wind_15 & 1 & -14.330 & 1.212 & 14.382 & 242 & 19 & 75.7 \\
\hline 106 Wind_06 & 10 Riser_A & 1 & 14.183 & -0.951 & 14.215 & 240 & 19 & 74.8 \\
\hline 107 Wind_07 & 108 Wind_08 & 1 & -9.687 & 0.500 & 9.700 & 163 & 13 & 74.6 \\
\hline 112 Wind_12 & 10 Riser_A & 1 & 21.281 & -1.927 & 21.368 & 361 & 29 & 73.7 \\
\hline 112 Wind_12 & 113 Wind_13 & 1 & -20.288 & 0.807 & 20.304 & 342 & 29 & 70.0 \\
\hline 108 Wind_08 & 109 Wind_09 & 1 & -7.884 & 0.220 & 7.887 & 133 & 13 & 60.7 \\
\hline 117 Wind_17 & 118 Wind_18 & 1 & -7.163 & 0.723 & 7.200 & 121 & 13 & 55.4 \\
\hline 103 Wind_03 & 104 Wind_04 & 1 & 7.111 & -0.576 & 7.134 & 120 & 13 & 54.9 \\
\hline 118 Wind_18 & 119 Wind_19 & 1 & -5.349 & 0.438 & 5.366 & 90 & 13 & 41.3 \\
\hline 102 Wind_02 & 103 Wind_03 & 1 & 5.263 & -0.151 & 5.266 & 88 & 13 & 40.5 \\
\hline 109 Wind_09 & 110 Wind_10 & 1 & -4.443 & 0.362 & 4.458 & 75 & 13 & 34.3 \\
\hline 119 Wind_19 & 120 Wind_20 & 1 & -2.675 & 0.223 & 2.684 & 45 & 13 & 20.6 \\
\hline 110 Wind_10 & 111 Wind_11 & 1 & -2.630 & 0.078 & 2.631 & 44 & 13 & 20.2 \\
\hline 101 Wind_01 & 102 Wind_02 & 1 & 1.853 & -0.432 & 1.903 & 32 & 13 & 14.6 \\
\hline
\end{tabular}


Table C6.1

Mission Wind Farm - 5000 kW / 25 kV

WindPACT Turbine Design Scaling Studies

Power Flow Bus Report (100\% Generation, 50 MW)

\begin{tabular}{|c|c|c|c|c|c|}
\hline \multicolumn{2}{|r|}{ Bus } & \multirow{2}{*}{$\begin{array}{c}\text { Base } \\
\mathrm{kV}\end{array}$} & Voltage & Generation & ration \\
\hline Number & Name & & P.U. & $P(M W)$ & $Q$ (MVAr) \\
\hline & Mission_115 & 115 & 1 & -48.539 & 11.9682 \\
\hline 201 & Turbine_01 & 2.4 & 1 & 5 & 1.28398 \\
\hline 202 & Turbine_02 & 2.4 & 1 & 5 & 0.11956 \\
\hline 203 & Turbine_03 & 2.4 & 1 & 5 & 0.31049 \\
\hline 204 & Turbine_04 & 2.4 & 1 & 5 & 0.26069 \\
\hline 205 & Turbine_05 & 2.4 & 1 & 5 & 0.12992 \\
\hline 206 & Turbine_06 & 2.4 & 1 & 5 & -0.0667 \\
\hline 207 & Turbine_07 & 2.4 & 1 & 5 & -0.2631 \\
\hline 208 & Turbine_08 & 2.4 & 1 & 5 & 0.2806 \\
\hline 209 & Turbine_09 & 2.4 & 1 & 5 & 0.08116 \\
\hline 210 & Turbine_10 & 2.4 & 1 & 5 & -0.1169 \\
\hline & & & & 50 & 2.0197 Total Wind Generation \\
\hline & & & & 1.461 & 13.9879 Total Losses \\
\hline & Mission_25 & 24.9 & 0.9832 & & \\
\hline 10 & Riser_A & 24.9 & 0.9931 & & \\
\hline 15 & Riser_B & 24.9 & 0.9933 & & \\
\hline 101 & Wind_01 & 24.9 & 0.9866 & & \\
\hline 102 & Wind_02 & 24.9 & 0.9956 & & \\
\hline 103 & Wind_03 & 24.9 & 0.9941 & & \\
\hline 104 & Wind_04 & 24.9 & 0.9945 & & \\
\hline 105 & Wind_05 & 24.9 & 0.9955 & & \\
\hline 106 & Wind_06 & 24.9 & 0.9970 & & \\
\hline 107 & Wind_07 & 24.9 & 0.9985 & & \\
\hline 108 & Wind_08 & 24.9 & 0.9943 & & \\
\hline 109 & Wind_09 & 24.9 & 0.9959 & & \\
\hline 110 & Wind_10 & 24.9 & 0.9974 & & \\
\hline
\end{tabular}


Table C6.2

Mission Wind Farm - 5000 kW / 25 kV

WindPACT Turbine Design Scaling Studies

Power Flow Bus Report (100\% Generation, 50 MW)

\begin{tabular}{|c|c|c|c|c|c|c|c|c|}
\hline From Bus & To Bus & Ckt & MW & MVar & MVA & AMPS & Norm & $\%$ Norm \\
\hline 1 Mission_115 & 2 Mission_25 & 1 & -48.539 & 11.968 & 49.993 & 1159 & 50 & 100.0 \\
\hline 104 Wind_0 $0 \overline{4}$ & 10 Riser_A- & 1 & 19.877 & -0.688 & 19.889 & 464 & 21 & 94.7 \\
\hline 10 Riser_A & 2 Mission_25 & 1 & 34.742 & -1.946 & 34.796 & 812 & 37 & 94.0 \\
\hline 15 Riser_B & 2 Mission_25 & 1 & 14.897 & -0.310 & 14.900 & 348 & 16 & 93.1 \\
\hline 102 Wind_02 & 103 Wind_03 & 1 & 9.948 & -1.384 & 10.044 & 234 & 13 & 77.3 \\
\hline 105 Wind_05 & 106 Wind_06 & 1 & -9.950 & 0.705 & 9.974 & 232 & 13 & 76.7 \\
\hline 108 Wind_08 & 109 Wind_09 & 1 & -9.951 & 0.413 & 9.960 & 232 & 13 & 76.6 \\
\hline 103 Wind_03 & 10 Riser_A & 1 & 14.911 & -1.259 & 14.964 & 349 & 21 & 71.3 \\
\hline 104 Wind_04 & 105 Wind_05 & 1 & -14.913 & 0.760 & 14.932 & 348 & 21 & 71.1 \\
\hline 108 Wind_08 & 15 Riser_B & 1 & 14.912 & -0.314 & 14.915 & 348 & 21 & 71.0 \\
\hline 101 Wind_01 & 201 Turbine_01 & 1 & -5.000 & -1.284 & 5.162 & 1242 & 8 & 64.5 \\
\hline 103 Wind_03 & 203 Turbine_03 & 1 & -5.000 & -0.311 & 5.010 & 1205 & 8 & 62.6 \\
\hline 108 Wind_08 & 208 Turbine_08 & 1 & -5.000 & -0.281 & 5.008 & 1205 & 8 & 62.6 \\
\hline 107 Wind_07 & 207 Turbine_07 & 1 & -5.000 & 0.263 & 5.007 & 1204 & 8 & 62.6 \\
\hline 104 Wind_04 & 204 Turbine_04 & 1 & -5.000 & -0.261 & 5.007 & 1204 & 8 & 62.6 \\
\hline 105 Wind_05 & 205 Turbine_05 & 1 & -5.000 & -0.130 & 5.002 & 1203 & 8 & 62.5 \\
\hline 102 Wind_02 & 202 Turbine_02 & 1 & -5.000 & -0.120 & 5.001 & 1203 & 8 & 62.5 \\
\hline 110 Wind_10 & 210 Turbine_10 & 1 & -5.000 & 0.117 & 5.001 & 1203 & 8 & 62.5 \\
\hline 109 Wind_09 & 209 Turbine_09 & 1 & -5.000 & -0.081 & 5.001 & 1203 & 8 & 62.5 \\
\hline 106 Wind_06 & 206 Turbine_06 & 1 & -5.000 & 0.067 & 5.000 & 1203 & 8 & 62.5 \\
\hline 101 Wind_01 & 102 Wind_0 02 & 1 & 4.969 & -1.311 & 5.139 & 120 & 9 & 57.5 \\
\hline 106 Wind_06 & 107 Wind_07 & 1 & -4.979 & 0.455 & 4.999 & 116 & 9 & 55.5 \\
\hline 109 Wind_09 & 110 Wind_10 & 1 & -4.979 & 0.308 & 4.988 & 116 & 9 & 55.4 \\
\hline
\end{tabular}


Table C7.1

Mission Wind Farm - 5000 kW / 35 kV

WindPACT Turbine Design Scaling Studies

Power Flow Bus Report (100\% Generation, 50 MW)

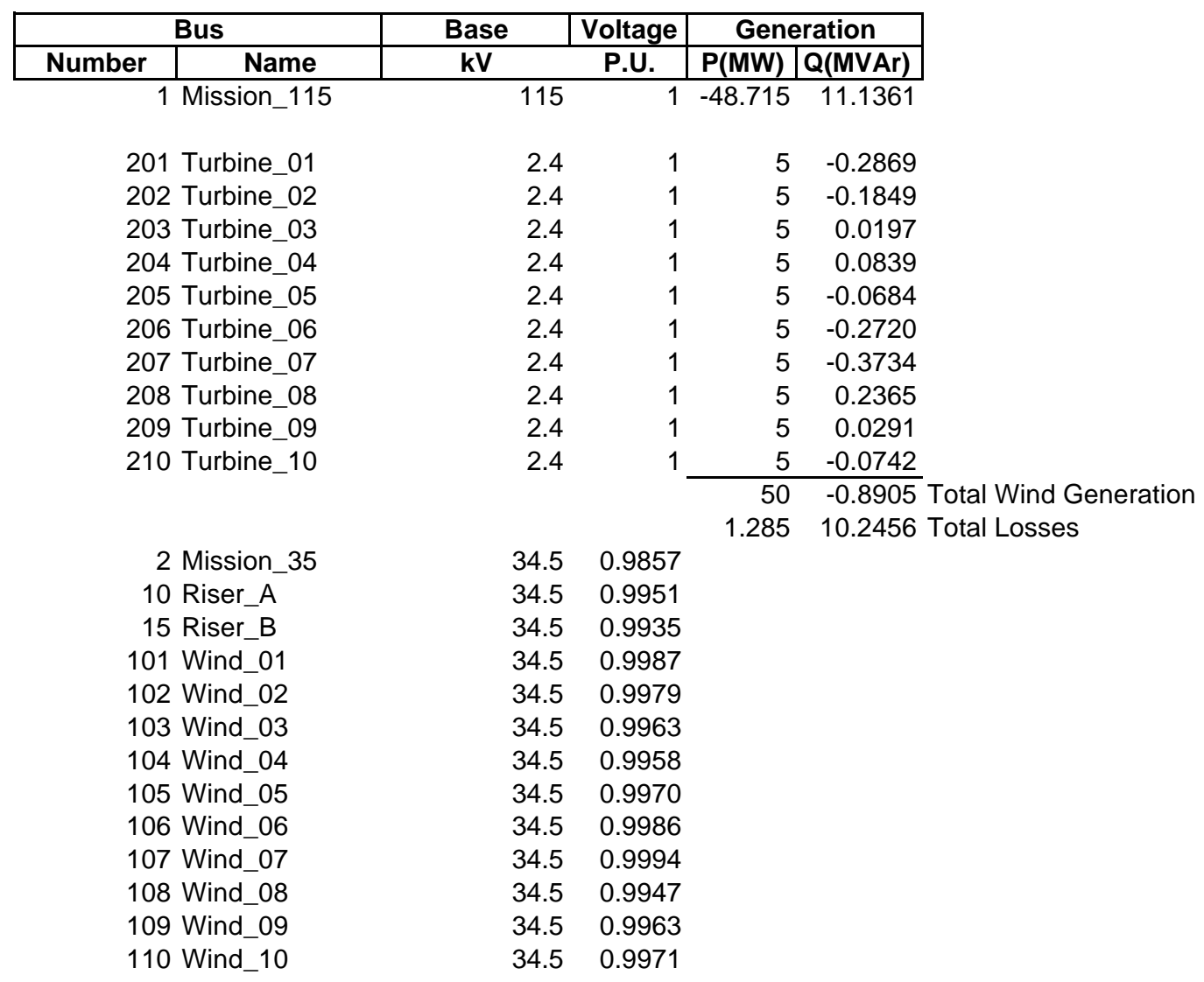


Table C7.2

Mission Wind Farm - 5000 kW / 35 kV

WindPACT Turbine Design Scaling Studies

Power Flow Bus Report (100\% Generation, 50 MW)

\begin{tabular}{|ccrrrrrrr}
\hline From Bus & To Bus & Ckt & MW & \multicolumn{1}{c}{ MVar } & MVA & AMPS & Norm & \%Norm \\
\hline 1 Mission_115 & 2 Mission_35 & 1 & -48.717 & 11.122 & 49.970 & 836 & 50 & 99.9 \\
15 Riser_B & 2 Mission_35 & 1 & 14.919 & -0.335 & 14.923 & 251 & 16 & 93.3 \\
1 10 Riser_A & 2 Mission_35 & 1 & 34.736 & -2.366 & 34.816 & 586 & 39 & 89.3 \\
104 Wind_04 & 105 Wind_05 & 1 & -15.205 & 1.134 & 15.247 & 256 & 19 & 80.2 \\
103 Wind_03 & 10 Riser_A & 1 & 15.111 & -0.953 & 15.141 & 254 & 19 & 79.7 \\
105 Wind_05 & 106 Wind_06 & 1 & -10.141 & 0.931 & 10.184 & 171 & 13 & 78.3 \\
102 Wind_02 & 103 Wind_03 & 1 & 10.107 & -0.827 & 10.141 & 170 & 13 & 78.0 \\
108 Wind_08 & 15 Riser_B & 1 & 14.761 & -0.241 & 14.763 & 248 & 19 & 77.7 \\
108 Wind_08 & 109 Wind_09 & 1 & -9.985 & 0.419 & 9.994 & 168 & 13 & 76.9 \\
104 Wind_04 & 10 Riser_A & 1 & 20.120 & -1.264 & 20.159 & 339 & 29 & 69.5 \\
107 Wind_07 & 207 Turbine_07 & 1 & -5.001 & 0.380 & 5.015 & 1206 & 8 & 62.7 \\
101 Wind_01 & 201 Turbine_01 & 1 & -5.000 & 0.289 & 5.009 & 1205 & 8 & 62.6 \\
106 Wind_06 & 206 Turbine_06 & 1 & -4.999 & 0.276 & 5.007 & 1204 & 8 & 62.6 \\
108 Wind_08 & 208 Turbine_08 & 1 & -4.999 & -0.231 & 5.005 & 1204 & 8 & 62.6 \\
102 Wind_02 & 202 Turbine_02 & 1 & -4.999 & 0.185 & 5.002 & 1203 & 8 & 62.5 \\
104 Wind_04 & 204 Turbine_04 & 1 & -5.000 & -0.088 & 5.001 & 1203 & 8 & 62.5 \\
105 Wind_05 & 205 Turbine_05 & 1 & -5.000 & 0.068 & 5.001 & 1203 & 8 & 62.5 \\
109 Wind_09 & 209 Turbine_09 & 1 & -5.000 & -0.023 & 5.000 & 1203 & 8 & 62.5 \\
103 Wind_03 & 203 Turbine_03 & 1 & -5.000 & -0.023 & 5.000 & 1203 & 8 & 62.5 \\
110 Wind_10 & 210 Turbine_10 & 1 & -4.999 & 0.081 & 5.000 & 1203 & 8 & 62.5 \\
106 Wind_06 & 107 Wind_07 & 1 & -5.088 & 0.524 & 5.115 & 86 & 13 & 39.3 \\
101 Wind_01 & 102 Wind_02 & 1 & 5.058 & -0.422 & 5.076 & 85 & 13 & 39.0 \\
109 Wind_09 & 110 Wind_10 & 1 & -5.024 & 0.318 & 5.034 & 84 & 13 & 38.7
\end{tabular}


Table C8.1

Mission Wind Farm - 10000 kW / 25 kV

WindPACT Turbine Design Scaling Studies

Power Flow Bus Report (100\% Generation, 50 MW)

\begin{tabular}{|c|c|c|c|c|c|c|}
\hline \multicolumn{2}{|c|}{ Bus } & \multirow{2}{*}{$\begin{array}{c}\text { Base } \\
\text { kV }\end{array}$} & \multirow{2}{*}{\begin{tabular}{|c|} 
Voltage \\
P.U.
\end{tabular}} & \multicolumn{2}{|c|}{ Generation } & \\
\hline Number & Name & & & $\mathbf{P}(\mathbf{M W})$ & Q(MVAr) & \\
\hline & sion_115 & 115 & 1 & -48.718 & 11.2604 & \\
\hline 201 & bine_01 & 4.16 & 1 & 10 & -0.05 & \\
\hline 202 & bine_02 & 4.16 & 1 & 10 & 0.31523 & \\
\hline 203 & bine_03 & 4.16 & 1 & 10 & 0.40656 & \\
\hline 204 & bine_04 & 4.16 & 1 & 10 & 0.26225 & \\
\hline 205 & bine_05 & 4.16 & 1 & 10 & -0.1021 & \\
\hline & & & & 50 & 0.8319 & Total Wind Generation \\
\hline & & & & 1.282 & 12.0923 & Total Losses \\
\hline & sion_25 & 24.9 & 0.9853 & & & \\
\hline 10 & er_A & 24.9 & 0.9915 & & & \\
\hline 101 & dd_01 & 24.9 & 0.9955 & & & \\
\hline 102 & d__02 & 24.9 & 0.9934 & & & \\
\hline 103 & d__03 & 24.9 & 0.9928 & & & \\
\hline 104 & dd_04 & 24.9 & 0.9937 & & & \\
\hline 105 & dd_05 & 24.9 & 0.9958 & & & \\
\hline
\end{tabular}


Table C8.2

Mission Wind Farm - 10000 kW / 25 kV

WindPACT Turbine Design Scaling Studies

Power Flow Bus Report (100\% Generation, 50 MW)

\begin{tabular}{|ccrrrrrrr|}
\hline From Bus & To Bus & Ckt & \multicolumn{1}{c|}{ MW } & MVar & MVA & AMPS & Norm & \%Norm \\
\hline 1 Mission_115 & 2 Mission_25 & 1 & -48.72 & 11.26 & 50.0 & 1159 & 50 & 100.0 \\
10 Riser_A & 2 Mission_25 & 1 & 49.53 & -2.12 & 49.6 & 1159 & 50 & 99.2 \\
104 Wind_04 & 10 Riser_A & 1 & 19.85 & -1.01 & 19.9 & 464 & 21 & 94.6 \\
102 Wind_02 & 10 Riser_A & 1 & 19.85 & -0.90 & 19.9 & 464 & 21 & 94.6 \\
103 Wind_03 & 203 Turbine_03 & 1 & -10.00 & -0.41 & 10.0 & 1389 & 11 & 91.0 \\
102 Wind_02 & 202 Turbine_02 & 1 & -10.00 & -0.32 & 10.0 & 1389 & 11 & 91.0 \\
104 Wind_04 & 204 Turbine_04 & 1 & -10.00 & -0.26 & 10.0 & 1388 & 11 & 90.9 \\
105 Wind_05 & 205 Turbine_05 & 1 & -10.00 & 0.10 & 10.0 & 1388 & 11 & 90.9 \\
101 Wind_01 & 201 Turbine_01 & 1 & -10.00 & 0.05 & 10.0 & 1388 & 11 & 90.9 \\
104 Wind_04 & 105 Wind_05 & 1 & -9.93 & 0.69 & 10.0 & 232 & 13 & 76.6 \\
101 Wind_01 & 102 Wind_02 & 1 & 9.93 & -0.63 & 10.0 & 232 & 13 & 76.6 \\
103 Wind_03 & 10 Riser_A & 1 & 9.93 & -0.17 & 9.9 & 232 & 13 & 76.4
\end{tabular}


Table C9.1

Mission Wind Farm - 10000 kW / 35 kV

WindPACT Turbine Design Scaling Studies

Power Flow Bus Report (100\% Generation, 50 MW)

\begin{tabular}{|c|c|c|c|c|c|c|}
\hline \multicolumn{2}{|r|}{ Bus } & Base & Voltage & \multicolumn{2}{|c|}{ Generation } & \\
\hline Number & Name & kV & P.U. & $\mathrm{P}(\mathrm{MW})$ & $Q$ (MVAr) & \\
\hline \multicolumn{2}{|r|}{1 Mission_115 } & 115 & 1 & -48.944 & 10.2475 & \\
\hline \multicolumn{2}{|c|}{201 Turbine 01} & 4.16 & 1 & 10 & 0.01402 & \\
\hline \multicolumn{2}{|c|}{202 Turbine_02 } & 4.16 & 1 & 10 & 0.36814 & \\
\hline \multicolumn{2}{|c|}{203 Turbine 03} & 4.16 & 1 & 10 & 0.29878 & \\
\hline \multicolumn{2}{|c|}{204 Turbine 04} & 4.16 & 1 & 10 & 0.34261 & \\
\hline \multirow{3}{*}{\multicolumn{2}{|c|}{205 Turbine_05 }} & 4.16 & 1 & 10 & -0.0112 & \\
\hline & & & & 50 & 1.0124 & Total Wind Generation \\
\hline & & & & 1.056 & 11.2599 & Total Losses \\
\hline \multicolumn{2}{|r|}{2 Mission 35} & 34.5 & 0.9883 & & & \\
\hline \multicolumn{2}{|c|}{10 Riser_A } & 34.5 & 0.9917 & & & \\
\hline \multicolumn{2}{|c|}{101 Wind_01 } & 34.5 & 0.9949 & & & \\
\hline \multicolumn{2}{|c|}{102 Wind_02 } & 34.5 & 0.9927 & & & \\
\hline \multicolumn{2}{|c|}{103 Wind_03 } & 34.5 & 0.9931 & & & \\
\hline \multicolumn{2}{|c|}{104 Wind_04 } & 34.5 & 0.9928 & & & \\
\hline \multicolumn{2}{|c|}{105 Wind_05 } & 34.5 & 0.9951 & & & \\
\hline
\end{tabular}


Table C9.2

Mission Wind Farm - 10000 kW / 35 kV

WindPACT Turbine Design Scaling Studies

Power Flow Bus Report (100\% Generation, 50 MW)

\begin{tabular}{|c|c|c|c|c|c|c|c|c|}
\hline From Bus & To Bus & Ckt & MW & MVar & MVA & AMPS & Norm & $\%$ Norm \\
\hline 1 Mission 115 & 2 Mission 35 & 1 & -48.94 & 10.25 & 50.01 & 837 & 50 & 100.0 \\
\hline 10 Riser A & 2 Mission 35 & 1 & 49.55 & -2.10 & 49.59 & 837 & 50 & 98.9 \\
\hline 102 Wind_02 & 202 Turbine_02 & 1 & -10.00 & -0.37 & 10.01 & 1389 & 29 & 91.1 \\
\hline 104 Wind_04 & 204 Turbine_04 & 1 & -10.00 & -0.34 & 10.01 & 1389 & 29 & 91.0 \\
\hline 103 Wind_03 & 203 Turbine_03 & 1 & -10.00 & -0.30 & 10.00 & 1388 & 11 & 90.9 \\
\hline 101 Wind_01 & 201 Turbine_01 & 1 & -10.00 & -0.01 & 10.00 & 1388 & 11 & 90.9 \\
\hline 105 Wind 05 & 205 Turbine 05 & 1 & -10.00 & 0.01 & 10.00 & 1388 & 11 & 90.9 \\
\hline 104 Wind_04 & 105 Wind_05 & 1 & -9.93 & 0.64 & 9.95 & 167 & 11 & 76.7 \\
\hline 101 Wind_01 & 102 Wind_02 & 1 & 9.93 & -0.62 & 9.95 & 167 & 11 & 76.6 \\
\hline 103 Wind 03 & 10 Riser A & 1 & 9.93 & -0.33 & 9.94 & 167 & 13 & 76.4 \\
\hline 104 Wind_04 & 10 Riser_A & 1 & 19.84 & -0.92 & 19.86 & 335 & 13 & 68.6 \\
\hline 102 Wind_02 & 10 Riser_A & 1 & 19.84 & -0.86 & 19.86 & 335 & 13 & 68.5 \\
\hline
\end{tabular}


Table D1 Short-Circuit Fault Current Evaluation

Table D2 Short-Circuit Results

Table D3 Short-Circuit Results Alternate Plan

Table D4 Short-Circuit Input 


\section{APPENDIX D \\ SHORT-CIRCUIT STUDY RESULTS}

CAI made a short-circuit calculation to determine whether standard equipment ratings were applicable and to provide short-circuit contributions to the utility system. For this analysis, we assumed the utility $115-\mathrm{kV}$ system equivalent provided a three-phase fault level of 50,000 amps. If required, the utility can provide a more accurate short-circuit equivalent for its system, though this was not necessary for this study.

The primary short-circuit concern is whether the $15-\mathrm{kV}-35-\mathrm{kV}$ reclosers will have sufficient interrupting capability when applied to a generating system. The symmetrical interrupting rating of a standard VWE recloser is 12,000 amps. Table D1 summarizes the fault currents at various locations for the $750-\mathrm{kW} 15-\mathrm{kV}$ plan. In general, fault currents on the $15-\mathrm{kV}$ system exceed the 12,000 -amp level at all riser locations. The fault-current contribution from the $115-\mathrm{kV}$ system is high, primarily due to its proximity (approximately 1,000 feet). The fault currents at the riser locations for the $25-\mathrm{kV}$ and $35-\mathrm{kV}$ plans, while less than those for the $15-\mathrm{kV}$ plan, were also higher than 12,000 amps.

Modifications to lower fault currents could be made to the substation if a wind farm were to be located very close to a transmission line. Solutions could include: installation of either a highimpedance distribution transformer, installation of a reactor, or purchase of higher-rated switching devices.

A second short-circuit study with a slightly modified electrical system was conducted to show the effect of fault currents of a wind farm located 10,000 feet, rather than 1,000 feet, from a $115 / 15-\mathrm{kV}$ substation. The results from this study are also tabulated in Table D1. In the modified electrical system, all of the fault currents on the $15-\mathrm{kV}$ system are less than the maximum standard-equipment ratings.

Additional fine-tuning of the short-circuit analysis should be performed once an actual wind farm site has been determined. In addition, the utility to which the wind farm will be connected will need to know the additional fault contribution from the farm. 


\section{Table D1}

\section{WindPACT Turbine Design Scaling Studies Short Circuit Fault Current Evaluation 750 kW - 15 kV Plan}

\begin{tabular}{|c|c|c|c|c|c|c|}
\hline \multirow[b]{3}{*}{ Bus Number } & \multirow[b]{3}{*}{ Bus Name } & \multirow[b]{3}{*}{ kV } & \multicolumn{4}{|c|}{ Fault Current (kA) } \\
\hline & & & \multicolumn{2}{|c|}{ Plan As In Figure 1} & \multicolumn{2}{|c|}{$\begin{array}{l}\text { Alternative Plan with } \\
10,000 \text { ft. extra OH } \\
\text { Distribution Line }\end{array}$} \\
\hline & & & $\begin{array}{l}\text { Three- } \\
\text { Phase } \\
\end{array}$ & $\begin{array}{l}\text { Line-to- } \\
\text { Ground }\end{array}$ & $\begin{array}{l}\text { Three- } \\
\text { Phase }\end{array}$ & $\begin{array}{l}\text { Line-to- } \\
\text { Ground }\end{array}$ \\
\hline 2 & Mission 13.8 & 13.8 & 25.8 & 20.7 & 15.0 & 15.0 \\
\hline 10 & Riser A & 13.8 & 14.1 & 5.8 & 9.9 & 5.2 \\
\hline 15 & Riser B & 13.8 & 17.5 & 9.0 & 10.1 & 7.2 \\
\hline 20 & Riser C & 13.8 & 15.5 & 7.4 & 10.0 & 6.3 \\
\hline 25 & Riser D & 13.8 & 21.8 & 13.5 & 10.3 & 9.2 \\
\hline 30 & Riser E & 13.8 & 22.3 & 13.6 & 11.3 & 9.8 \\
\hline 149 & Wind 49 & 13.8 & 19.3 & 9.8 & 10.9 & 7.8 \\
\hline 249 & Turbine 49 & 0.69 & 29.4 & 25.8 & 28.8 & 25.5 \\
\hline 166 & Wind 66 & 13.8 & 7.8 & 4.1 & 6.6 & 4.0 \\
\hline 266 & Turbine 66 & 0.69 & 28.7 & 25.5 & 27.7 & 25.2 \\
\hline
\end{tabular}




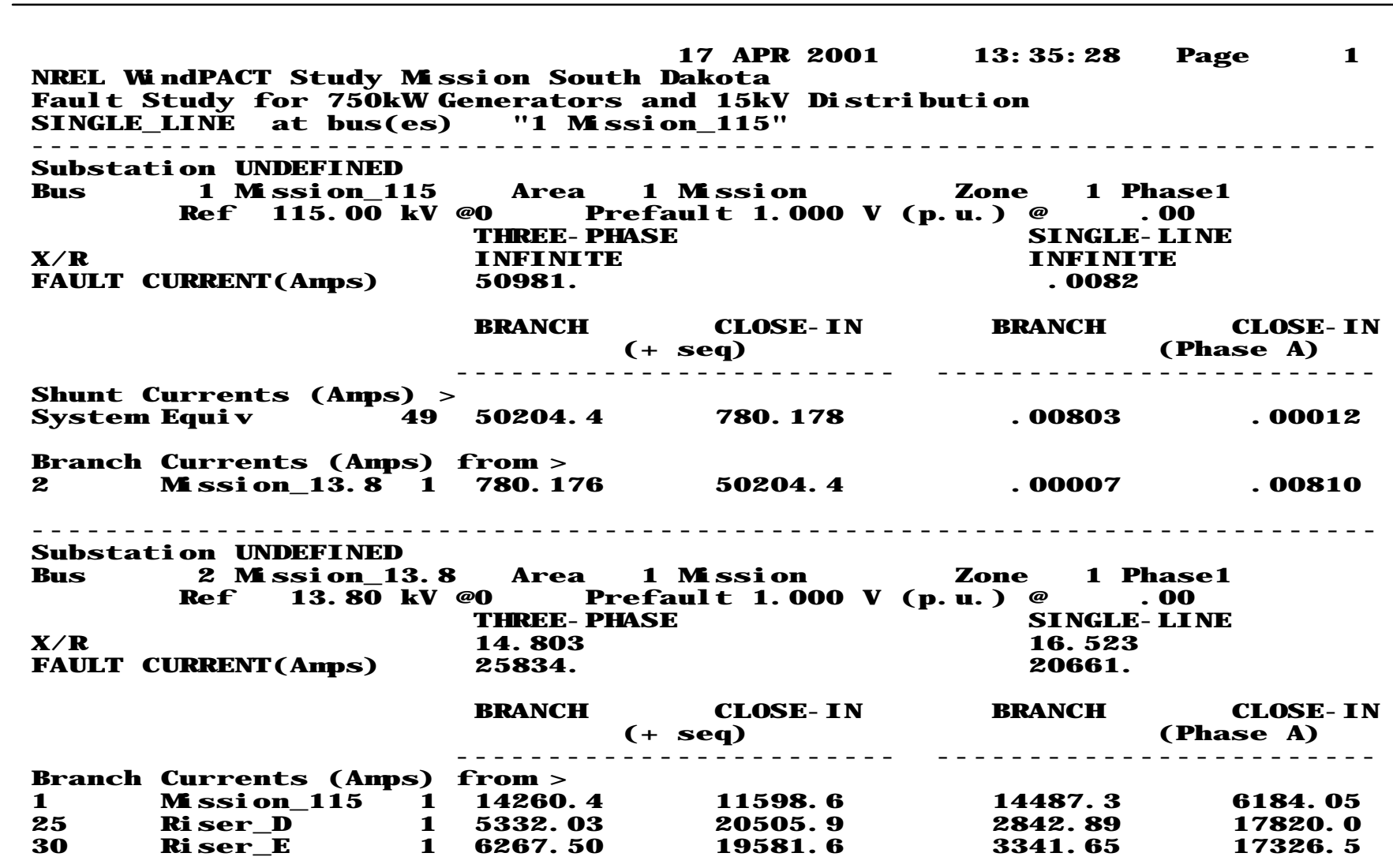


17 APR 2001 13: 35: 28 Page 2

NREL W NdPACT St udy $M$ ssi on South Dakota

Faul $t$ St udy for $750 \mathrm{~kW}$ Generat ors and $15 \mathrm{kV}$ Di st $r$ i but $i$ on

SI NGLE_LI NE at bus(es) "10 Ri ser_A"

Subst at i on UNDEFI NED

Bus $10 \mathrm{Ri}$ ser A Ref 13.80 kV @D Area 1 M ssion THREE- PHASE

$X / R$ 7. 7349

FAULT CURRENT( Amps) 14183.

Zone 1 Phasel

(p.u.) @ 1 .OO SI NGLE- LI NE 4. 7423 5786. 4

BRANCH

CLOSE- I N

$$
(+ \text { seq })
$$

\begin{tabular}{|c|c|c|c|c|c|c|}
\hline & & & BRANCH & $\begin{array}{l}\text { CLOSE- I N } \\
\text { eq) }\end{array}$ & BRANCH & $\begin{array}{l}\text { CLOSE- I } \\
\operatorname{ase~A)~}\end{array}$ \\
\hline $\begin{array}{l}\mathrm{Branch} \\
15 \\
111 \\
112\end{array}$ & $\begin{array}{l}\text { Cur rents } \\
\text { Ri ser B } \\
\text { W nd } \overline{1} 1 \\
\text { W nd } 12\end{array}$ & $\begin{array}{r}\text { ( Amps ) } \\
1 \\
1 \\
1\end{array}$ & $\begin{array}{l}\text { f rom > } \\
9174.58 \\
2411.83 \\
2617.21\end{array}$ & $\begin{array}{l}5028.95 \\
11780.0 \\
11572.8\end{array}$ & $\begin{array}{l}4423.35 \\
655.999 \\
711.861\end{array}$ & $\begin{array}{l}1367.84 \\
5132.71 \\
5076.38\end{array}$ \\
\hline
\end{tabular}

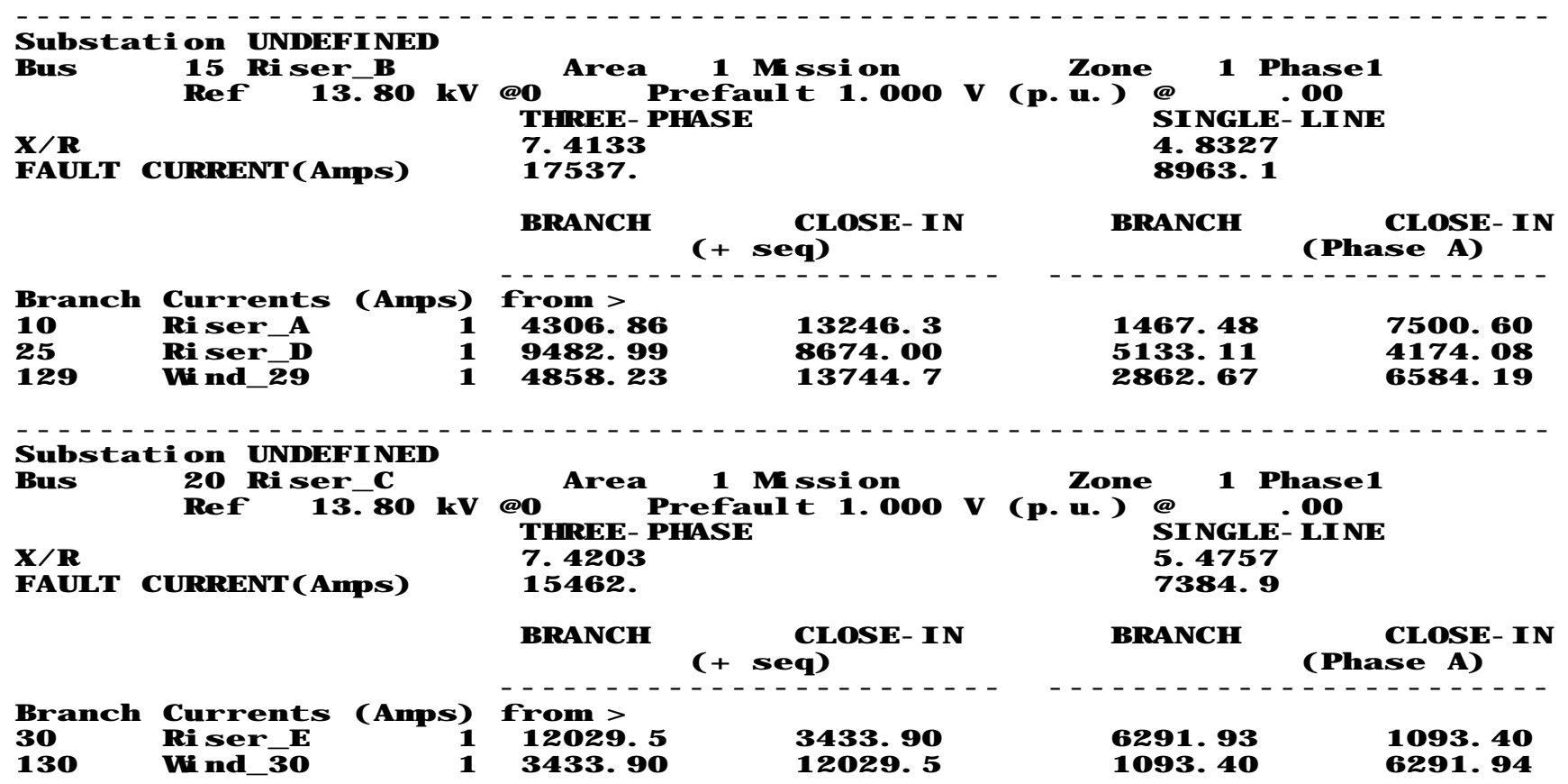


17 APR 2001

13: $35: 28$

Page

3

NREL Wi ndPACT St udy $M$ ssi on Sout h Dakot a

Faul t St udy for $750 \mathrm{~kW}$ Gener at ors and $15 \mathrm{kV}$ Di st $\mathrm{r}$ i but i on

SI NGLE_LI NE at bus(es) " 25 Ri ser_D'

Subst at i on UNDEFI NED

Bus $25 \mathrm{Ri}$ ser D Ref $13.80 \mathrm{kV}$ CD Area 1 M ssion THREE- PHASE

$X / R$ 10. 647

FAULT CURRENT( Amps) 21807.

Zone 1 Phasel ( p. u. ) @ NGLE- LI NE SI NGLE 13483.

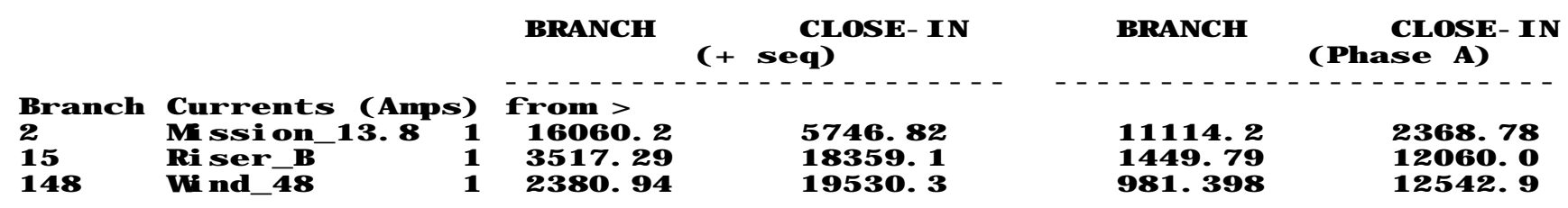

Subst at i on UNDEFI NED

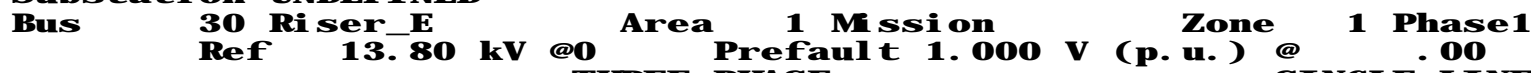

$X / R$ THREE- PHASE

FAULT CURRENT( Amps) 9. 8456 22338.

7. 6935 13616.

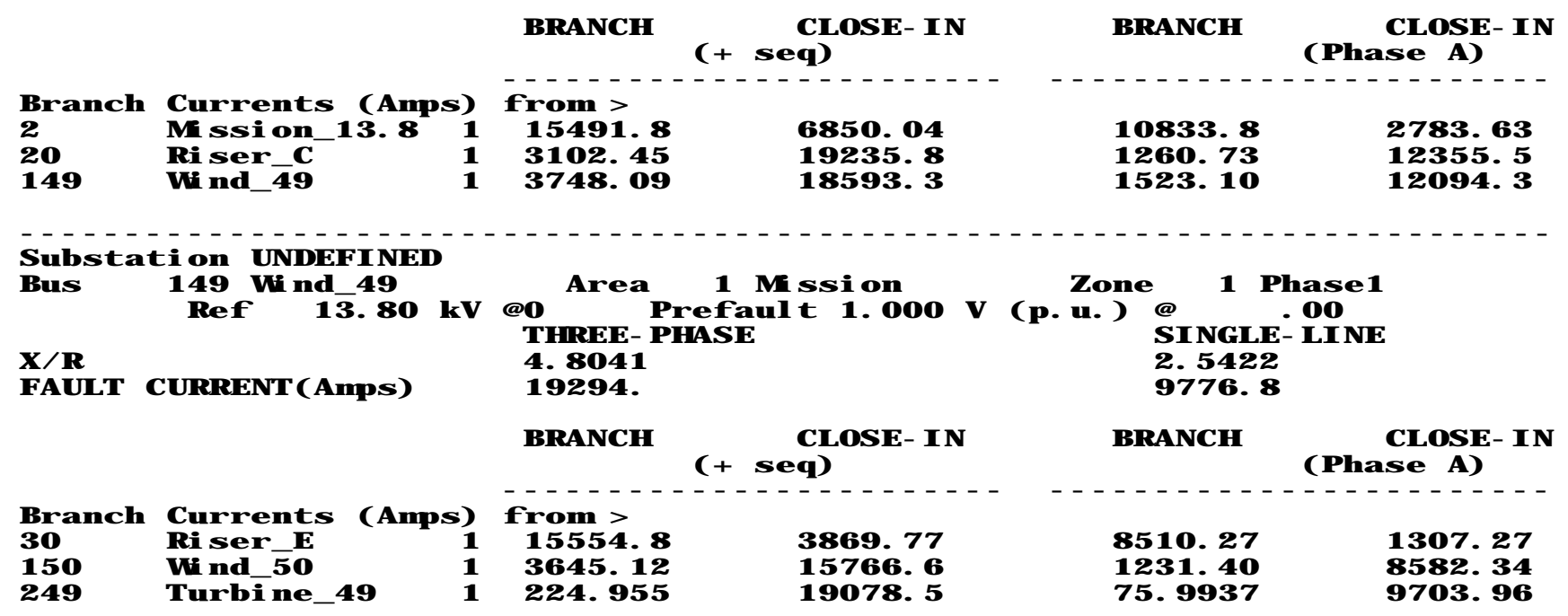


17 APR 2001 13: 35: 28 Page 4

NREL Wi ndPACT St udy $M$ ssi on Sout h Dakota

Faul $t$ St udy for $750 \mathrm{~kW}$ Gener at ors and $15 \mathrm{kV}$ Di st $\mathrm{r}$ i but i on

SI NGLE_LI NE at bus(es) " 249 Tur bi ne_49"

Subst at i on UNDEFI NED

Bus 249 Tur bi ne 49

Ref $4 \overline{8} \mathrm{kV}$ a Area $1 \mathrm{M}$ ssion

CDREE- PHASE

I NFI NI TE

29373.

Zone 1 Phasel

$X / R$

FAULT CURRENT( AmpS)

BRANCH CLOSE- I N $(+$ seq) SI NGLE- LI NE I NFI NI TE 25823.

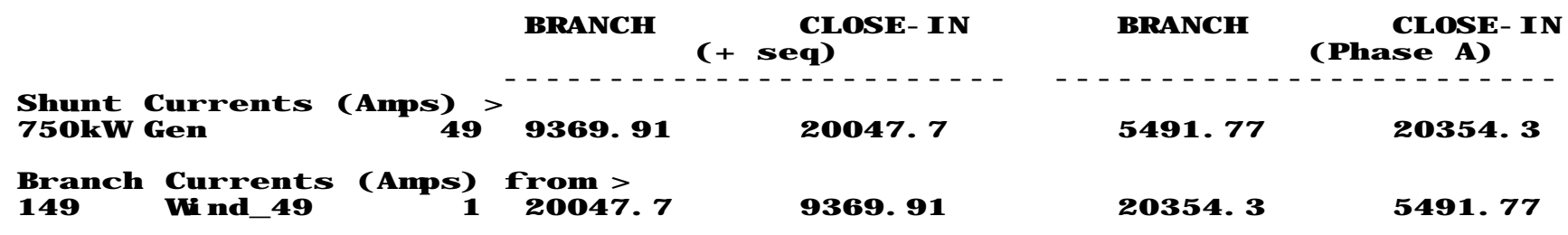

Subst at i on UNDEFI NED

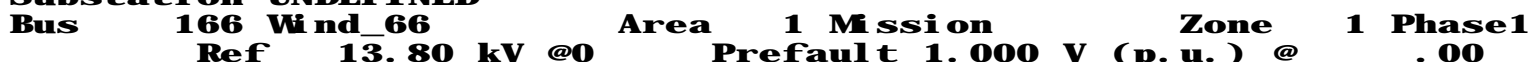
RHREE-PHASE

$\begin{array}{lll}X / R & & \text { S. } \\ \text { FAULT CURRENT( Amps) } & 77636 & 0051\end{array}$

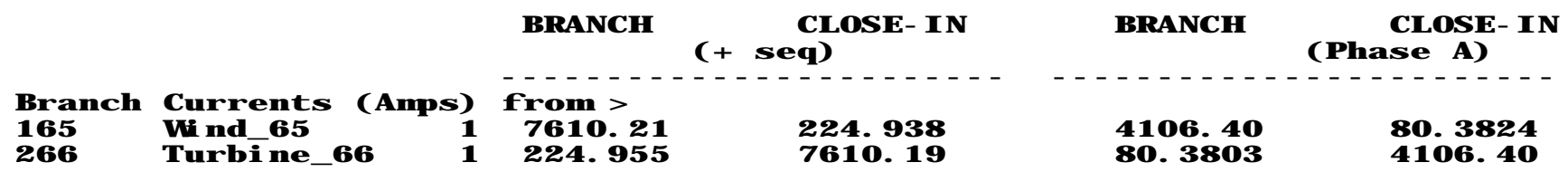


17 APR 2001 13: 35: 28 Page 5

NREL Wi ndPACT St udy $M$ ssi on Sout h Dakot a

Fault St udy for $750 \mathrm{~kW}$ Gener at ors and $15 \mathrm{kV}$ Di st $r$ i but $i$ on

SI NGLE_LI NE at bus(es) "266 Turbi ne_66"

Subst at i on UNDEFI NED

Bus 266 Turbi ne 66 Area 1 Mssion Zone 1 Phasel Ref $\quad 4 \overline{8} \mathrm{kV}$ @ 0 Prefaul $\mathrm{t} 1.000 \mathrm{~V}$ ( p. u.) @ .00

$X / R \quad$ THREE- PHASE SI NGLE-LI NE

$\begin{array}{lll}\text { FAULT CURRENT( Amps) } & 28675 . & 25457 .\end{array}$

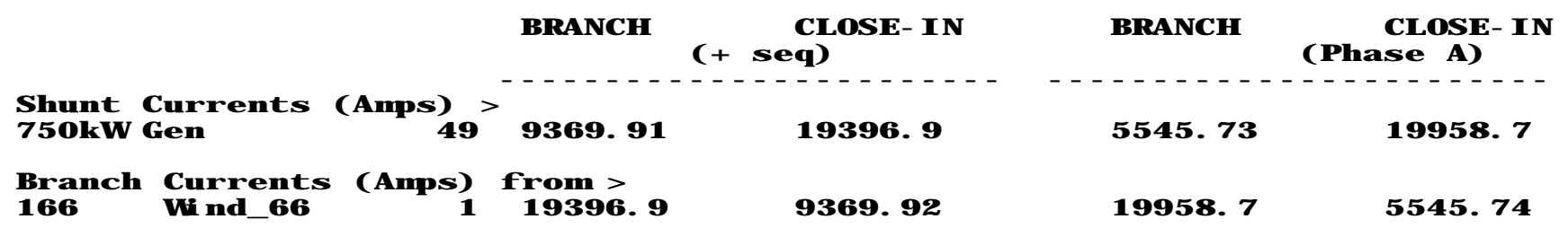


Table D3 Short Circuit Results Alternate Plan

NREL WindPACT St udy $M$ ssi on Sout h Dakot a

29 J UN 2000

14: 41: 11 Page

1

Faul $t$ St udy for $750 \mathrm{~kW}$ Generat ors and $15 \mathrm{kV}$ Di st $\mathrm{ri}$ but i on 10 , ooo kft. of Di st $r$ i but i on

SI NGLE_LI NE at bus(es) "I M ssion_115"

Subst at $\mathrm{i}$ on UNDEFI NED

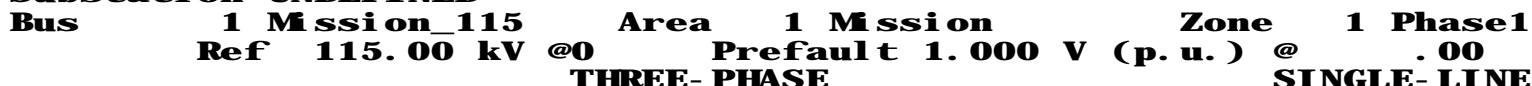

$X / R$

4. 0406

FAULT CURRENT( Amps)

2728. 1

SI NGLE- LI NE

I NFI NI TE

.0114

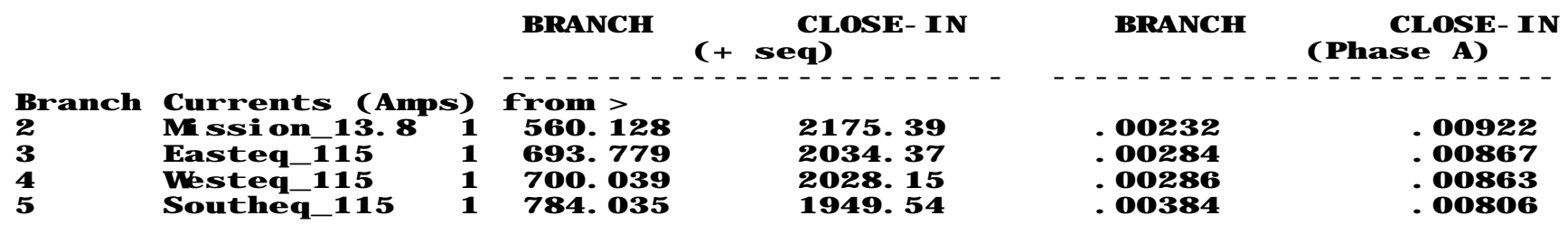

Subst at i on UNDEFI NED

Bus $2 \mathrm{Mssion} 13.8$ Area $1 \mathrm{Mssion}$ Zone 1 Phasel

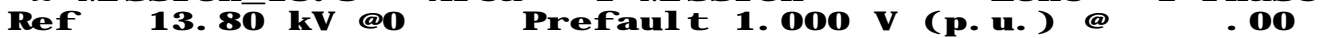

$X / R$

FAULT CURRENT( AmpS)

\section{THREE- PHASE}

6. 0048

15034.

BRANCH
CLOSE- I N $(+\mathrm{seq})$

Branch Currents (Amps) from $>$

$\begin{array}{llll}1 & \text { M ssion_115 } & 1 & 8256.55 \\ 25 & \text { Ri ser_D } & 1 & 3229.37 \\ 30 & \text { Ri ser_E } & 1 & 3548.07\end{array}$

(

6777.15

11804. 5
11485.8

29 J UN 2000

NREL Wi ndPACT St udy $M$ ssion Sout h Dakot a SI NGLE- LI NE 7. 8352 14961.

Faul t St udy for $750 \mathrm{~kW}$ Generat ors and $15 \mathrm{kV}$ Di st $\mathrm{r}$ i but i on SI NGLE_LI NE at bus(es) "3 Easteq_115" Subst at i on UNDEFI NED

Bus 3 East eq 115 3 East eq 115
Ref $115.00 \mathrm{kV}$ (⿻) Area 1 M ssion THREE- PHASE

X/ R I NFI NI TE

FAULT CURRENT( AmpS) 50707.

BRANCH

CLOSE- I N ( Phase A)

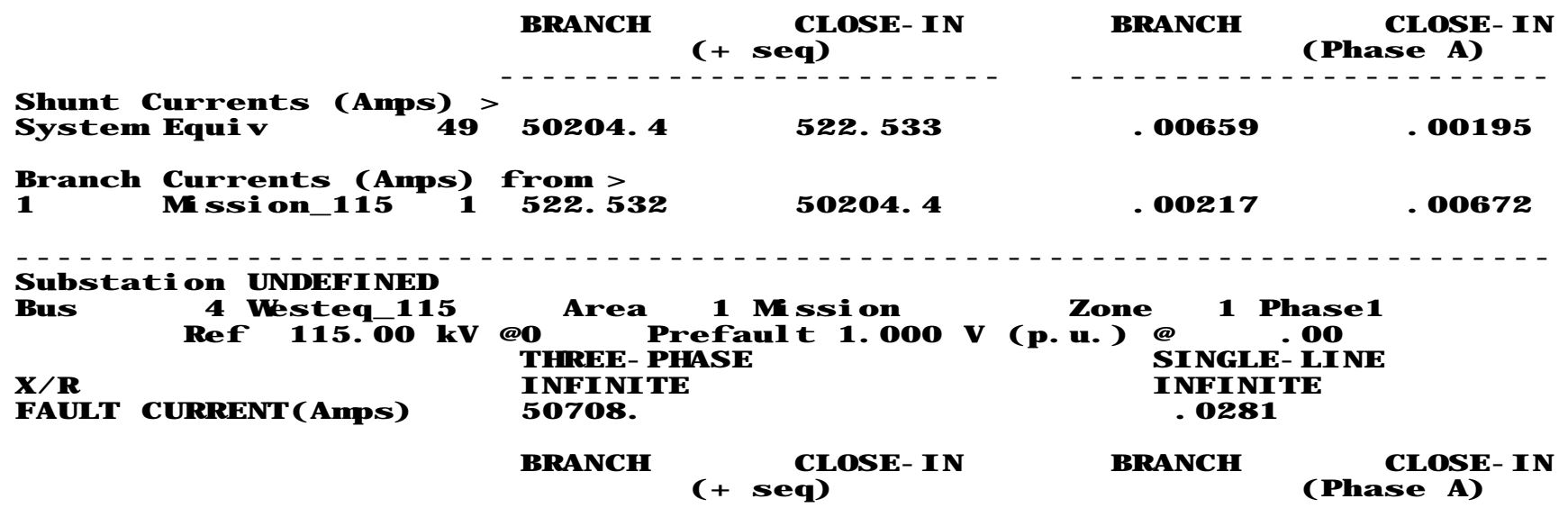


Table D3 Short Circuit Results Alternate Plan

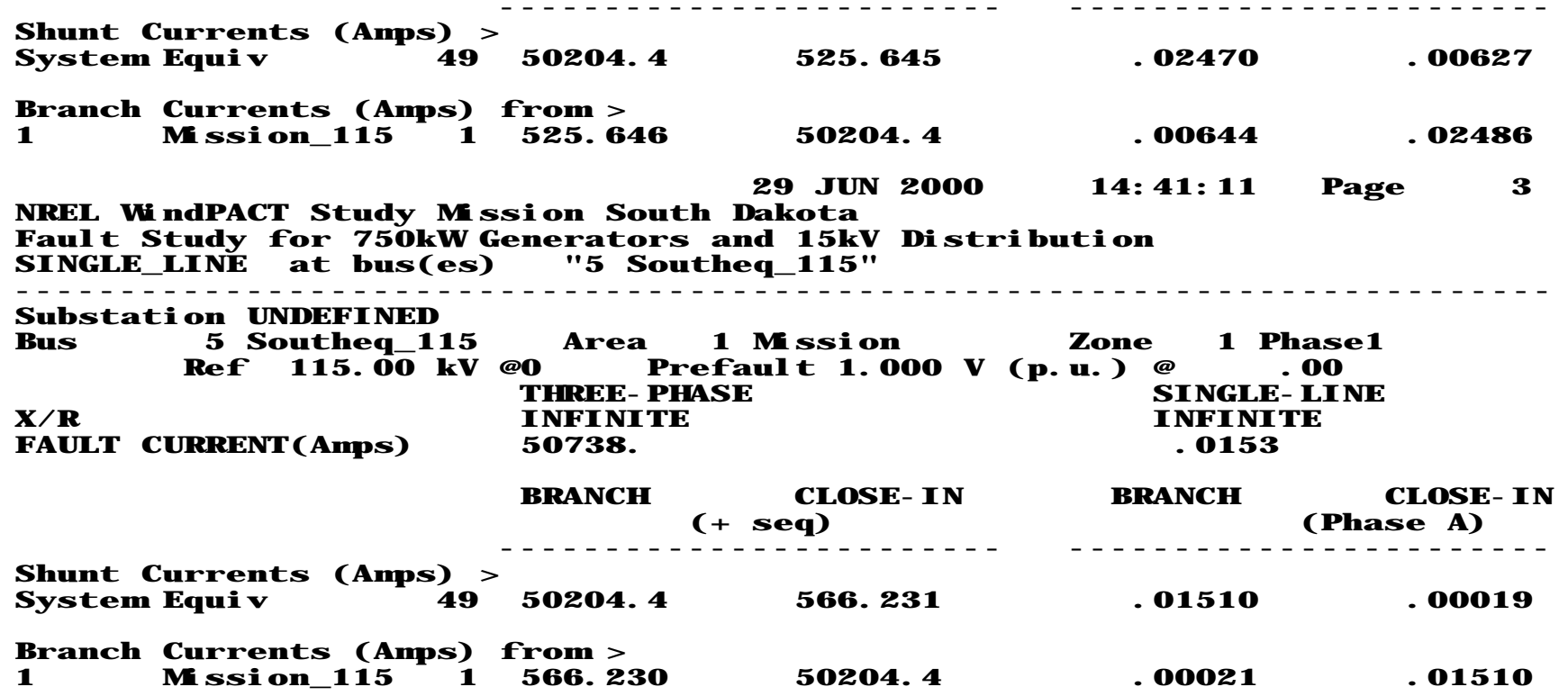

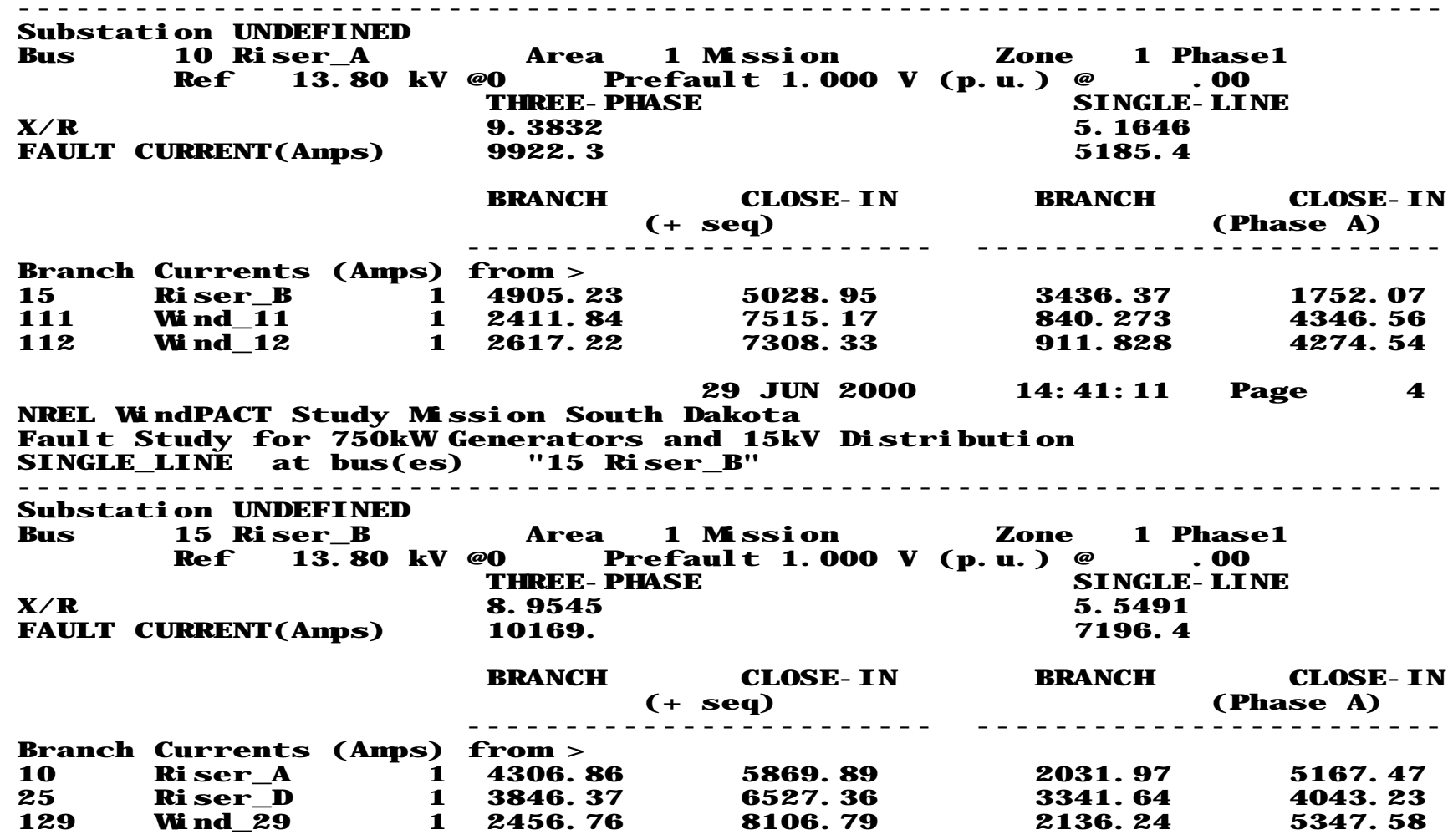

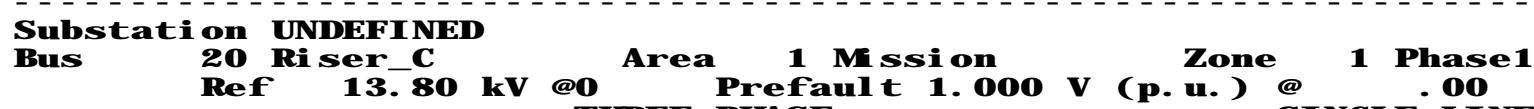

$\begin{array}{lll}X / R & \text { THREE-PHASE } & \text { SI NGLE- } \\ & 7.0027 & 5.5897\end{array}$ 
Table D3 Short Circuit Results Alternate Plan

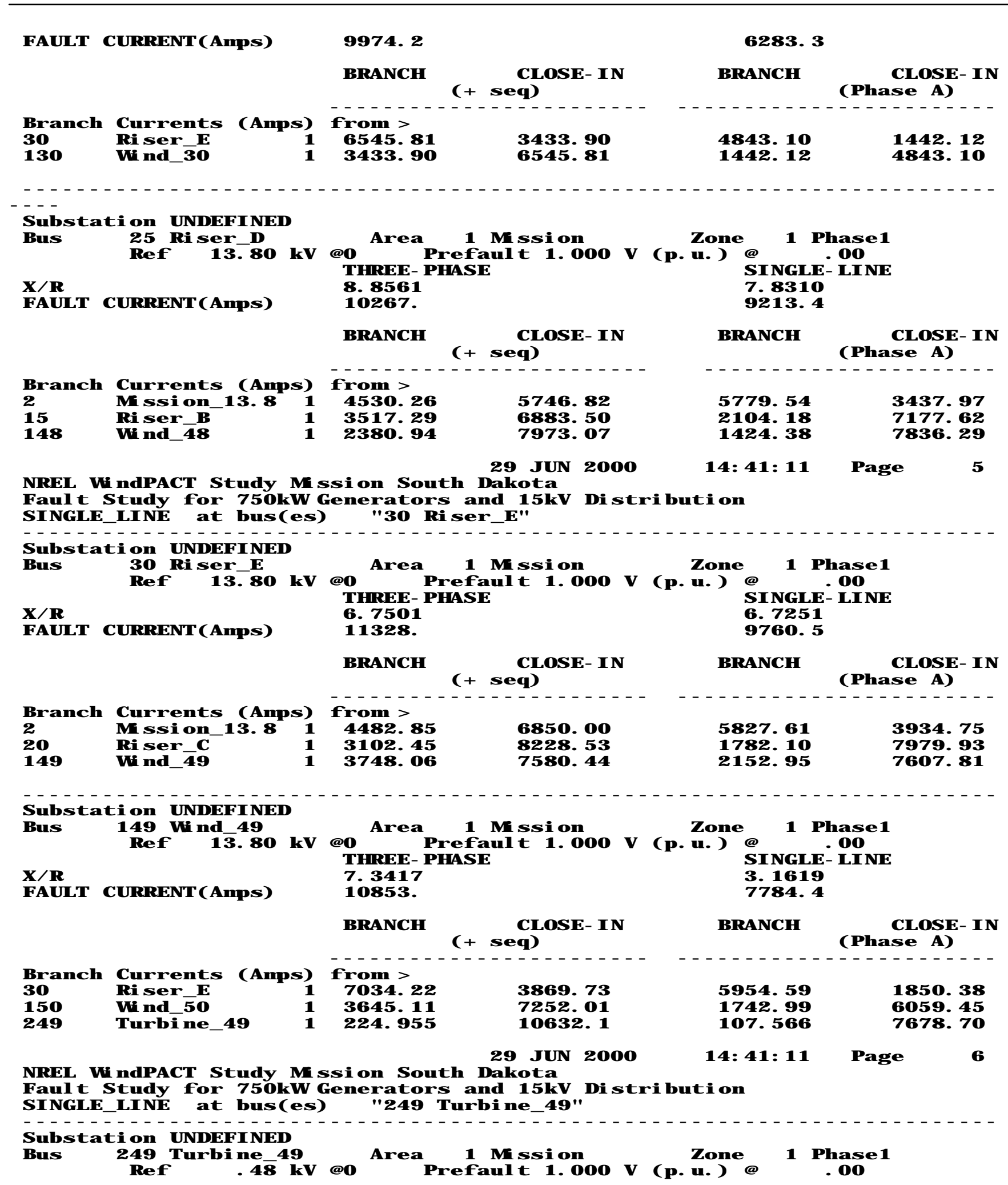


Table D3 Short Circuit Results Alternate Plan

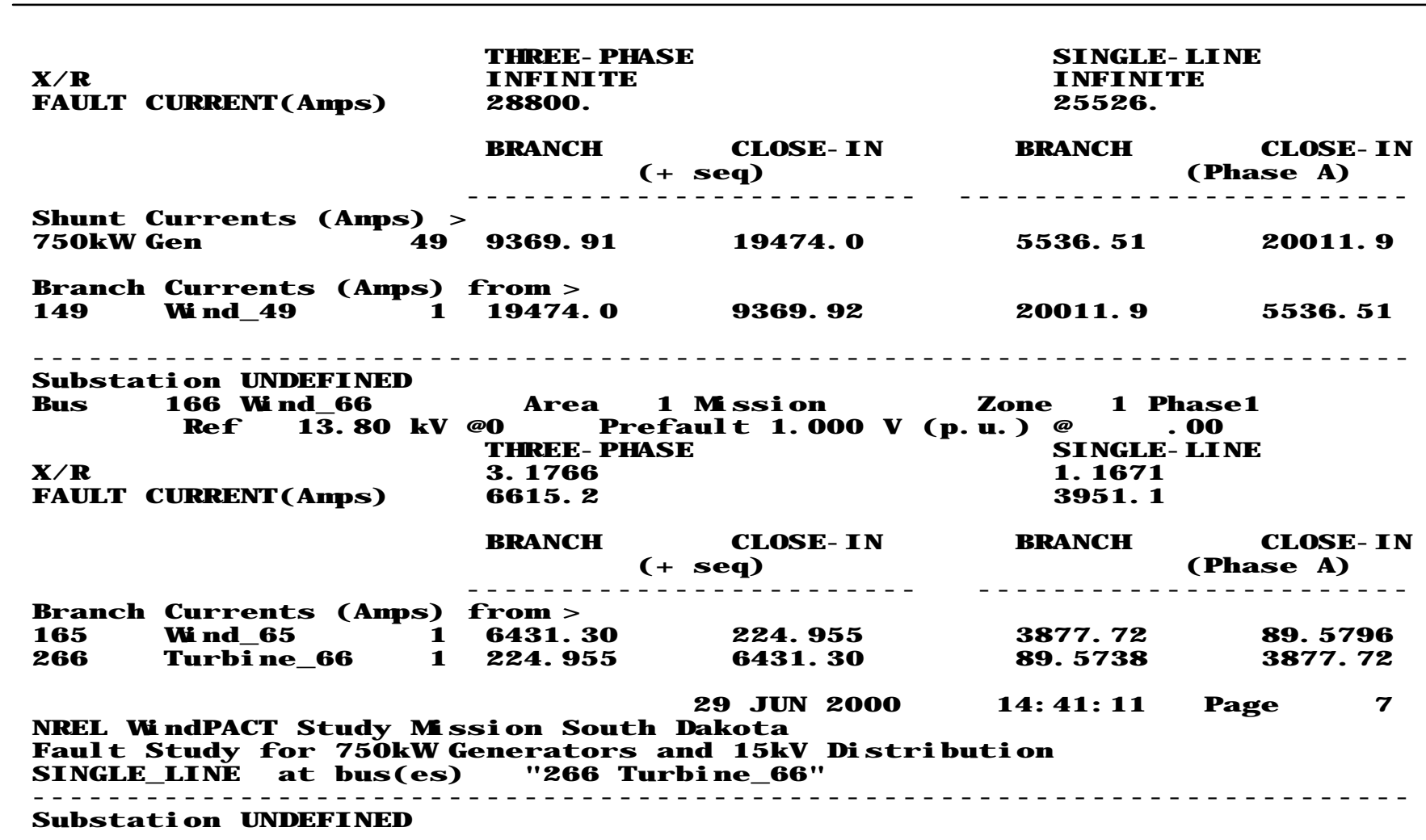


Table D4 Short Circuit Input

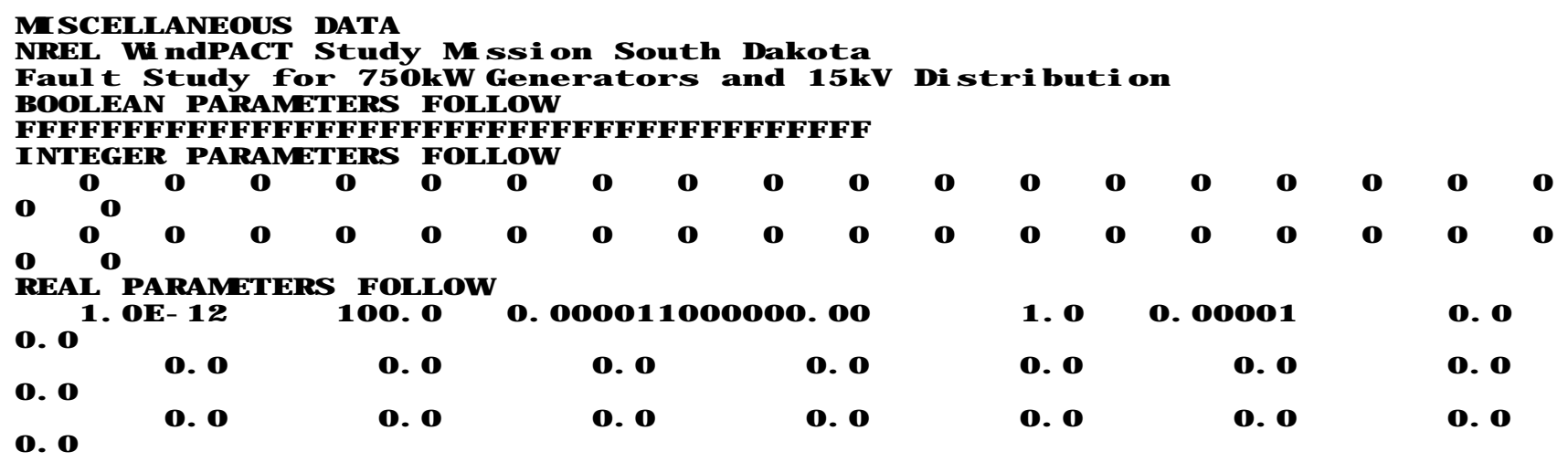

AREA CODES AND NAMES

1 Mssion M ssion Area

ZONE NAMES

1 Phasel

SHUNT CODES

1 Syst em Equi v

$2750 k W$ Gen

BUS DATA FOLLONS

549 M SSion_115

1

1 115. 000 1. 00000 0.000000

2 Msion_13.8

10 Ri ser_A

$15 \mathrm{Ri}$ ser $^{-} \mathrm{B}$

$20 \mathrm{Ri}^{2} \mathrm{ser}_{-}^{-} \mathrm{C}$

$25 \mathrm{Ri}^{-}$ser-D $^{-}$

$30 \mathrm{Ri}$ ser $\mathrm{E}$

$101 \mathrm{~W}$ nd $\overline{0} 1$

$102 \mathrm{~W}$ ind-o2

$103 \mathrm{~W}^{\mathrm{nd}} \mathrm{O} 3$

$104 \mathrm{~W}^{-} \mathrm{nd}-04$

$105 \mathrm{~W}$ nd-o5

$106 \mathrm{~W}^{-} \mathrm{nd} 06$

$107 \mathrm{~W}$ nd -07

$108 \mathrm{~W}$ nd-08

$109 \mathrm{~W}^{\mathrm{n}} \mathrm{-}-09$

$110 \mathrm{~W}^{-} \mathrm{nd}^{-10}$

$111 \mathrm{~W}^{-1} \mathrm{nd}^{-11}$

$112 \mathrm{~W}^{-1} \mathrm{nd}^{-12}$

$113 \mathrm{~W}^{-1} \mathrm{nd}^{-13}$

$114 \mathrm{~W}^{-1} \mathrm{nd}^{-14}$

$115 \mathrm{~W}^{-1} \mathrm{nd}^{-15}$

$116 \mathrm{~W}^{-1} \mathrm{nd}^{-16}$

$117 \mathrm{~W}$ nd-17

$118 \mathrm{~W}^{-1} \mathrm{nd}^{-18}$

$119 \mathrm{~W}^{-1} \mathrm{nd}^{-19}$

$120 \mathrm{~W}^{-} \mathrm{nd}^{-120}$

$121 \mathrm{~W}^{-} \mathrm{nd}^{-21}$

$122 \mathrm{~W}$ nd 22

$123 \mathrm{~W}^{\mathrm{nd}} \mathrm{-23}$

$124 \mathrm{~W}$ nd 24

$125 \mathrm{~W}$ ind 25

$126 \mathrm{~W}^{-1} \mathrm{nd}^{-2} 26$

$127 \mathrm{~W}$ nd-27

$128 \mathrm{~W}^{\mathrm{nd}} 28$

$129 \mathrm{~W}^{-1}$ nd 29

$130 \mathrm{~W}^{-1} \mathrm{nd}^{-} 30$

$131 \mathrm{~W}^{-} \mathrm{nd}^{-31}$

$132 \mathrm{~W}^{-} \mathrm{nd}^{-32}$

$133 \mathrm{~W}$ nd 33

$134 \mathrm{~W}^{\mathrm{nd}} \mathrm{nd}^{-3}$ - 100. 0000

o. 0 O. 0

o. 97400

$\begin{array}{lll}13.800 & 0.97400 & 8.021000 \\ 13.800 & 0.98320 & 11.856000\end{array}$

13. 800 0. 98080 10. 452000

$\begin{array}{lll}13.800 & 0.97410 & 9.814000\end{array}$

$\begin{array}{lll}13.800 & 0.97640 & 8.809000\end{array}$

$\begin{array}{lll}13.800 & 0.97340 \quad 8.886000\end{array}$

$\begin{array}{lll}13.800 & 1.00320 & 12.284000\end{array}$

13. 800 1. 00290 12. 277000

13. 800 1. 00230 12. 261000

13. 800 1. 00140 12. 239000

13. 800 1. 00020 12. 209000

$\begin{array}{lll}13.800 & 0.99870 & 12.173000\end{array}$

13. 800 0. 99690 12. 130000

13. 800 0. 99480 12. 082000

$\begin{array}{lll}13.800 & 0.99240 & 12.030000\end{array}$

13. 800 0. 98960 11. 974000

13.800 0. 98660 11. 916000

13. 800 0. 98680 11. 933000

13. 800 0. 99010 12. 008000

13.800 0. 99320 12. 080000

13. 800 0. 99580 12. 148000

13. 800 0. 99820 12. 211000

13. 800 1. 00030 12. 268000

13. 800 1. 00200 12. 319000

13. 8001.00350 12. 362000

13. 8001 1. 00470 12. 397000

13.8001 .00560 12. 423000

$13.800 \quad 1.00610 \quad 12.441000$

13. 800 1. 00640 12. 450000

13. 800 0. 98770 10. 471000

13. 800 0. 98740 10. 469000

13. 800 0. 98670 10. 467000

13. 800 0. 98570 10. 463000

13. 800 0. 98440 10. 459000

13. 800 0. 98280 10. 455000

13. 800 0. 97890 9. 931000

13. 800 0. 98330 10. 050000

13. 800 0. 98740 10. 169000

13. 800 0. 99120 10. 285000

13. 800 0. 99470 10. 397000
O

O

O

O

O

O

O

0

O

0

O

0

O

0

O

O

O

O

O

O

O

0

O

O

O

O

O

O

O 
Table D4 Short Circuit Input

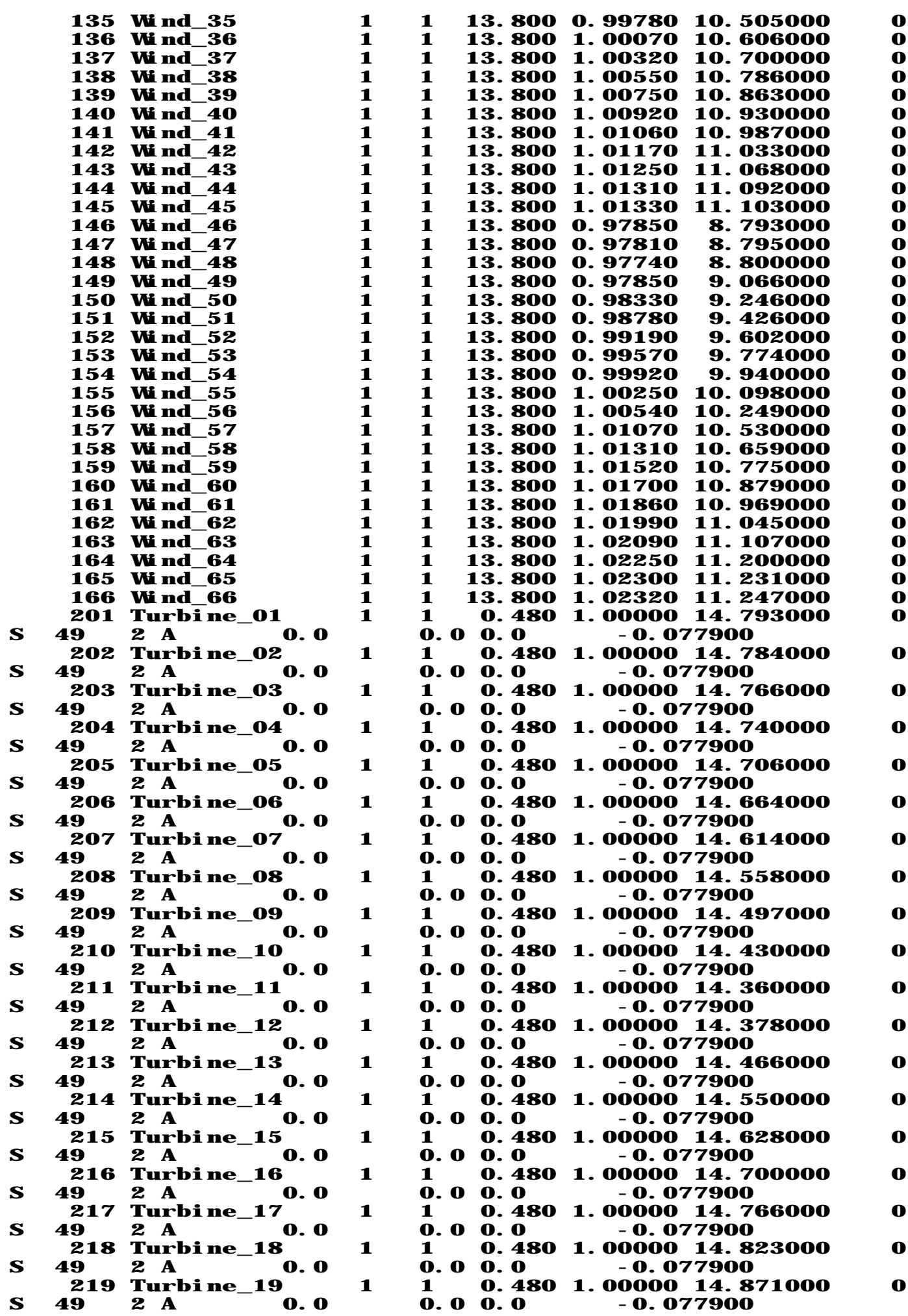


Table D4 Short Circuit Input

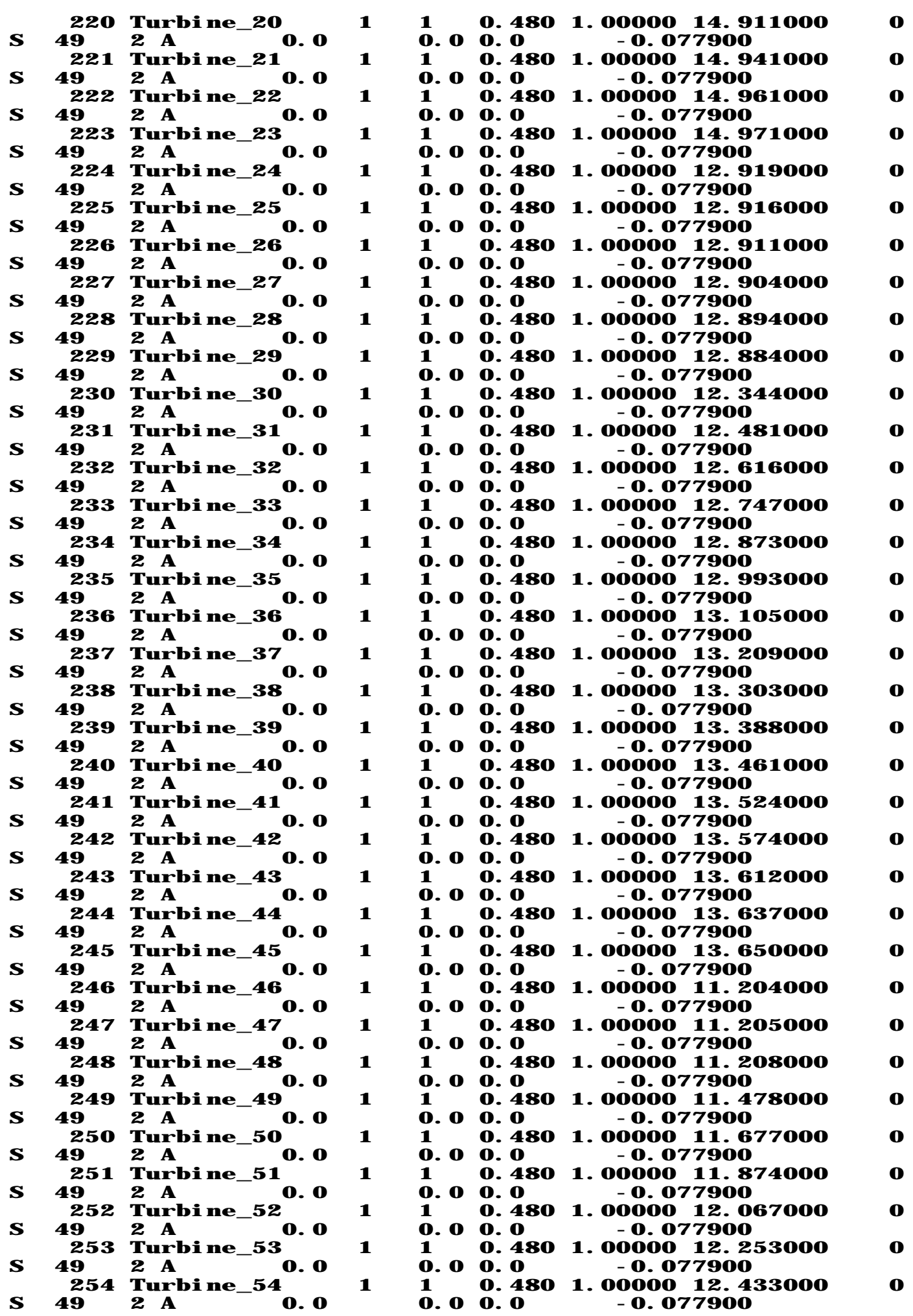


Table D4 Short Circuit Input

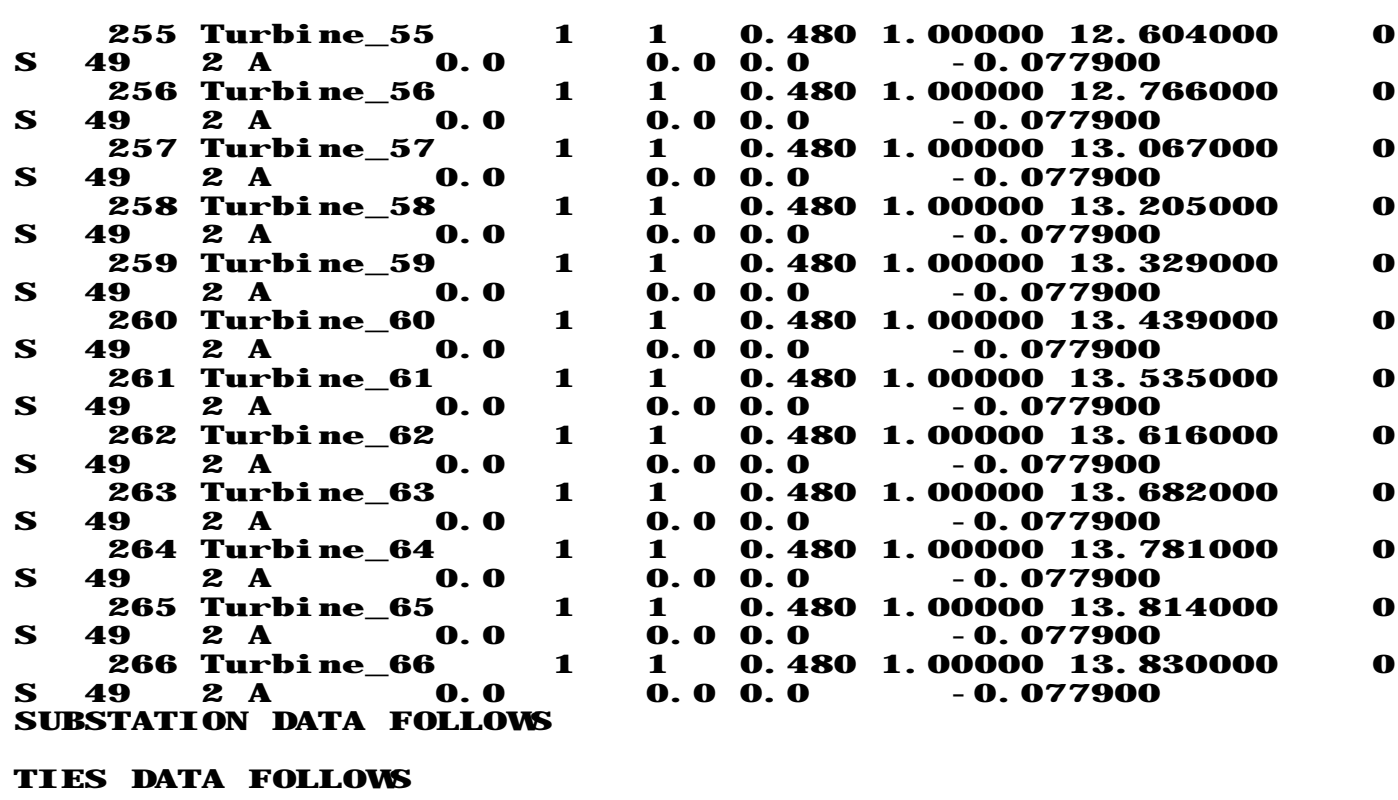

\begin{tabular}{|c|c|c|c|c|c|c|}
\hline $\mathrm{BR}$ & ANCH DATA & FOLLONS & & & & \\
\hline & 1 & 11 & 1 & O XFMR & & \\
\hline $\begin{array}{l}P \\
N \\
Z\end{array}$ & $\begin{array}{l}\text { O. } 01490 \\
\text { O. O1490 } \\
\text { NFI NI TE }\end{array}$ & $\begin{array}{l}\text { O. } 2833 \\
\text { O. } 2833\end{array}$ & $\begin{array}{l}\text { O. O } \\
\text { O. O } \\
\text { O. O }\end{array}$ & $\begin{array}{l}\text { O. } \mathrm{O} \\
\text { O. } \mathrm{O} \\
\text { O. } \mathrm{O}\end{array}$ & $\begin{array}{l}\text { O. O } \\
0.0 \\
.185\end{array}$ & $\begin{array}{r}\text { O. O } \\
\text { O. } \\
-3.520\end{array}$ \\
\hline - & 2 & 25 & . & O LI NE & & \\
\hline $\mathbf{P}$ & 0.0127 & 0.0555 & 0.0 & O. 0 & 0.0 & 0.0 \\
\hline$\stackrel{N}{7}$ & 0.0555 & 0. 0555 & 0.0 & O. 0 & 0.0 & 0.0 \\
\hline Z & ${ }_{15}^{0.066}$ & $25^{0.258}$ & 0.0 & O. $\mathrm{O}$ & & \\
\hline $\mathbf{P}$ & 0.0292 & o. 1275 & 0.0 & O. 0 & 0.0 & 0.0 \\
\hline $\mathbf{N}$ & 0.0292 & 0. 1275 & 0.0 & 0.0 & 0.0 & 0.0 \\
\hline Z & 0. 152 & O. 594 & O. 0 & O. 0 & & \\
\hline & 10 & $\begin{array}{lll}15 & 1 & 1\end{array}$ & 1 & O LI NE & & \\
\hline$P$ & 0. 0292 & O. 1371 & O. $\mathrm{O}$ & O. $\mathrm{O}$ & O. 0 & O. $\mathrm{O}$ \\
\hline $\mathbf{N}$ & 0.0292 & 0. 1371 & 0.0 & 0.0 & 0.0 & 0.0 \\
\hline Z & 0. 152 & O. 639 & O. O & O. $\mathrm{O}$ & & \\
\hline & & $\begin{array}{lll}30 & 1 & 1\end{array}$ & 1 & O LI NE & & \\
\hline $\mathbf{P}$ & 0.0127 & 0. 0555 & 0.0 & 0.0 & 0.0 & 0.0 \\
\hline $\begin{array}{l}N \\
Z\end{array}$ & $\begin{array}{r}0.0127 \\
0.066\end{array}$ & $\begin{array}{r}0.0555 \\
0.258\end{array}$ & $\begin{array}{l}0.0 \\
0.0\end{array}$ & $\begin{array}{l}\text { O. } 0 \\
\text { O. } 0\end{array}$ & 0.0 & O. 0 \\
\hline & 30 & $\begin{array}{lll}20 & 1 & 1\end{array}$ & 1 & O LI NE & & \\
\hline $\mathbf{P}$ & 0.0292 & 0. 1275 & 0.0 & 0.0 & 0.0 & 0.0 \\
\hline$\stackrel{N}{Z}$ & 0. 0292 & O. 1275 & O. $\mathrm{O}$ & O. 0 & 0.0 & 0.0 \\
\hline Z & 0. 152 & O. 594 & 0.0 & O. 0 & & \\
\hline & 10 & 111 & 1 & O LI NE & & \\
\hline $\mathbf{P}$ & 0.0420 & O. 0095 & O. 0 & O. 0 & O. 0 & 0.0 \\
\hline $\begin{array}{l}N \\
Z\end{array}$ & $\begin{array}{r}0.0420 \\
0.107\end{array}$ & $\begin{array}{r}0.0095 \\
0.047\end{array}$ & $\begin{array}{l}\text { O. } 0 \\
\text { O. } 0\end{array}$ & $\begin{array}{l}\text { O. } 0 \\
\text { O. } 0\end{array}$ & 0.0 & 0.0 \\
\hline & 10 & $\begin{array}{lll}112 & 1 & 1\end{array}$ & 1 & O LI NE & & \\
\hline $\mathbf{P}$ & 0.0420 & 0. 0095 & 0.0 & O. $\mathrm{O}$ & 0.0 & 0.0 \\
\hline$N$ & 0. 0420 & 0. 0095 & 0.0 & O. 0 & 0.0 & 0.0 \\
\hline Z & 0. 107 & 0.047 & O. 0 & O. 0 & & \\
\hline & 15 & $129 \quad 1$ & 1 & O LI NE & & \\
\hline $\mathbf{P}$ & 0.0420 & 0. 0095 & 0.0 & 0.0 & 0.0 & 0.0 \\
\hline$N$ & 0. 0420 & 0. 0095 & O. $\mathrm{O}$ & O. $\mathrm{O}$ & 0.0 & 0.0 \\
\hline Z & 0. 107 & 0.047 & 0.0 & O. $\mathrm{O}$ & & \\
\hline & 200 & $\begin{array}{lll}130 & 1\end{array}$ & 1 & $\mathrm{O}$ LI NE & & \\
\hline P & O. $042 C$ & 0 0.0095 & O. 0 & O. $\mathrm{O}$ & O. $\mathrm{O}$ & O. $\mathrm{O}$ \\
\hline $\begin{array}{l}\mathbf{N} \\
\mathrm{Z}\end{array}$ & $\begin{array}{l}\text { O. } 042( \\
0.107\end{array}$ & $\begin{array}{ll}0.0095 \\
0.047 \\
0.04\end{array}$ & $\begin{array}{l}\text { O. O } \\
\text { O. O }\end{array}$ & $\begin{array}{l}0.0 \\
0.0\end{array}$ & 0.0 & 0.0 \\
\hline & 25 & 1481 & 2 & O LI NE & & \\
\hline
\end{tabular}


Table D4 Short Circuit Input

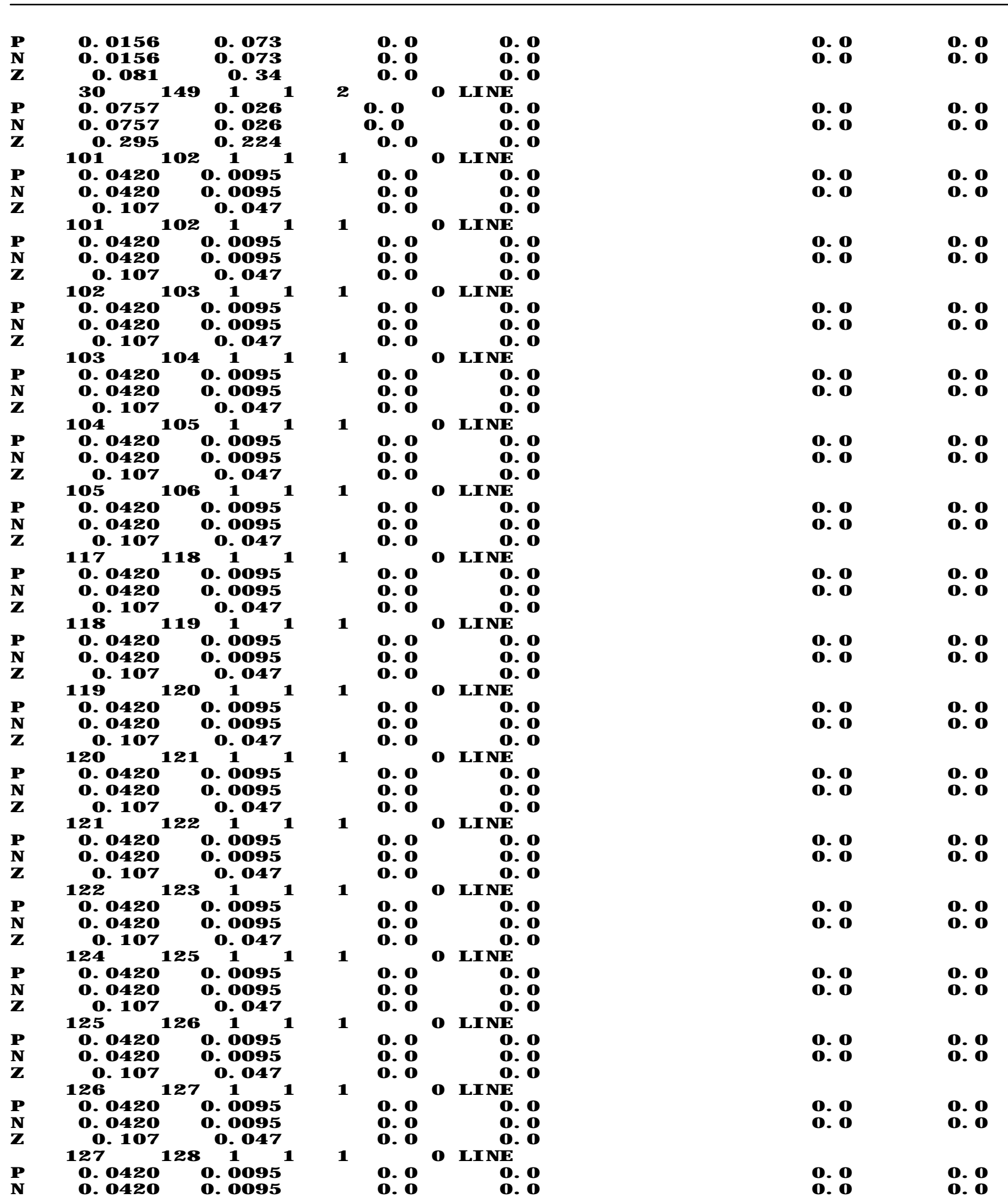




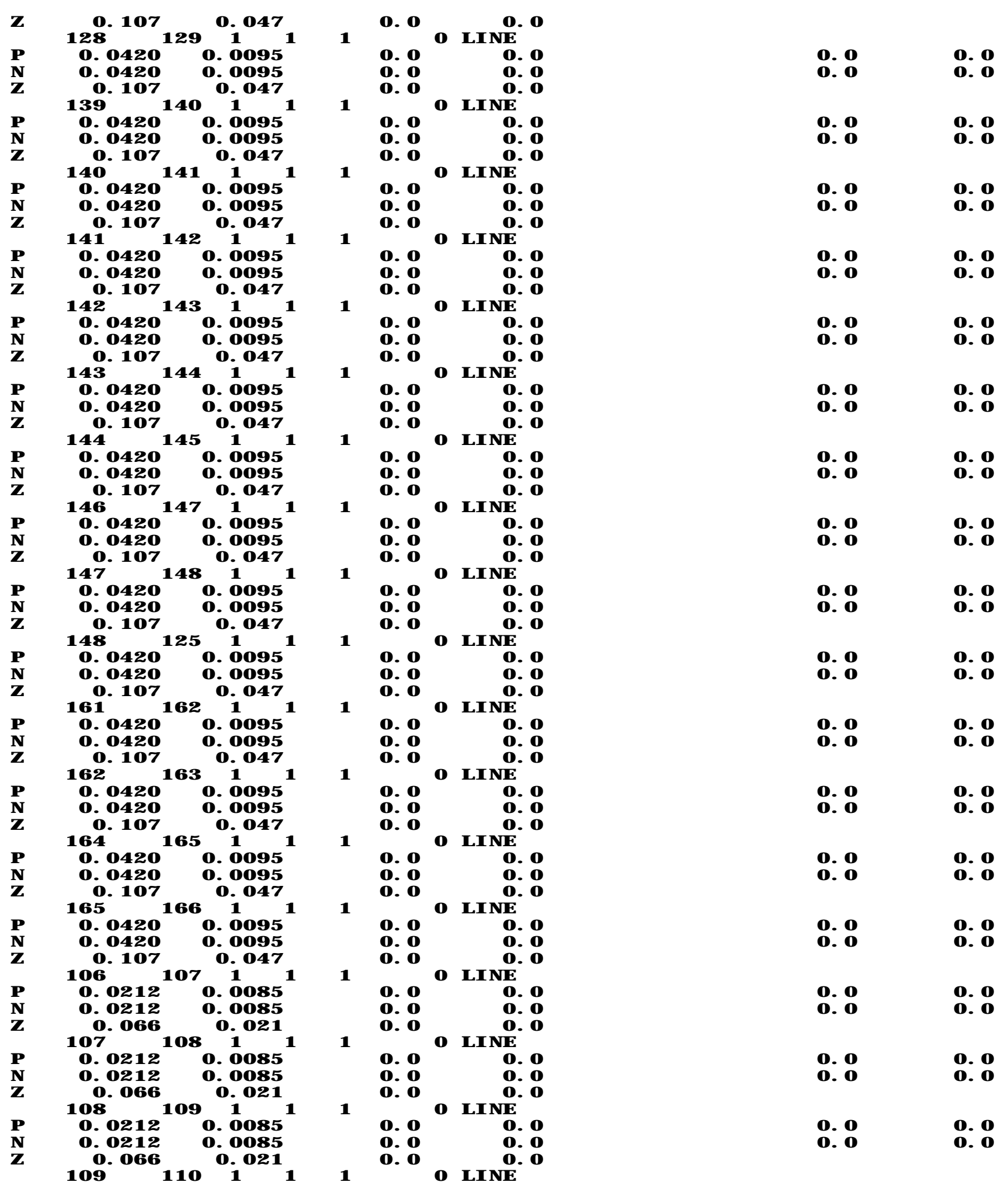




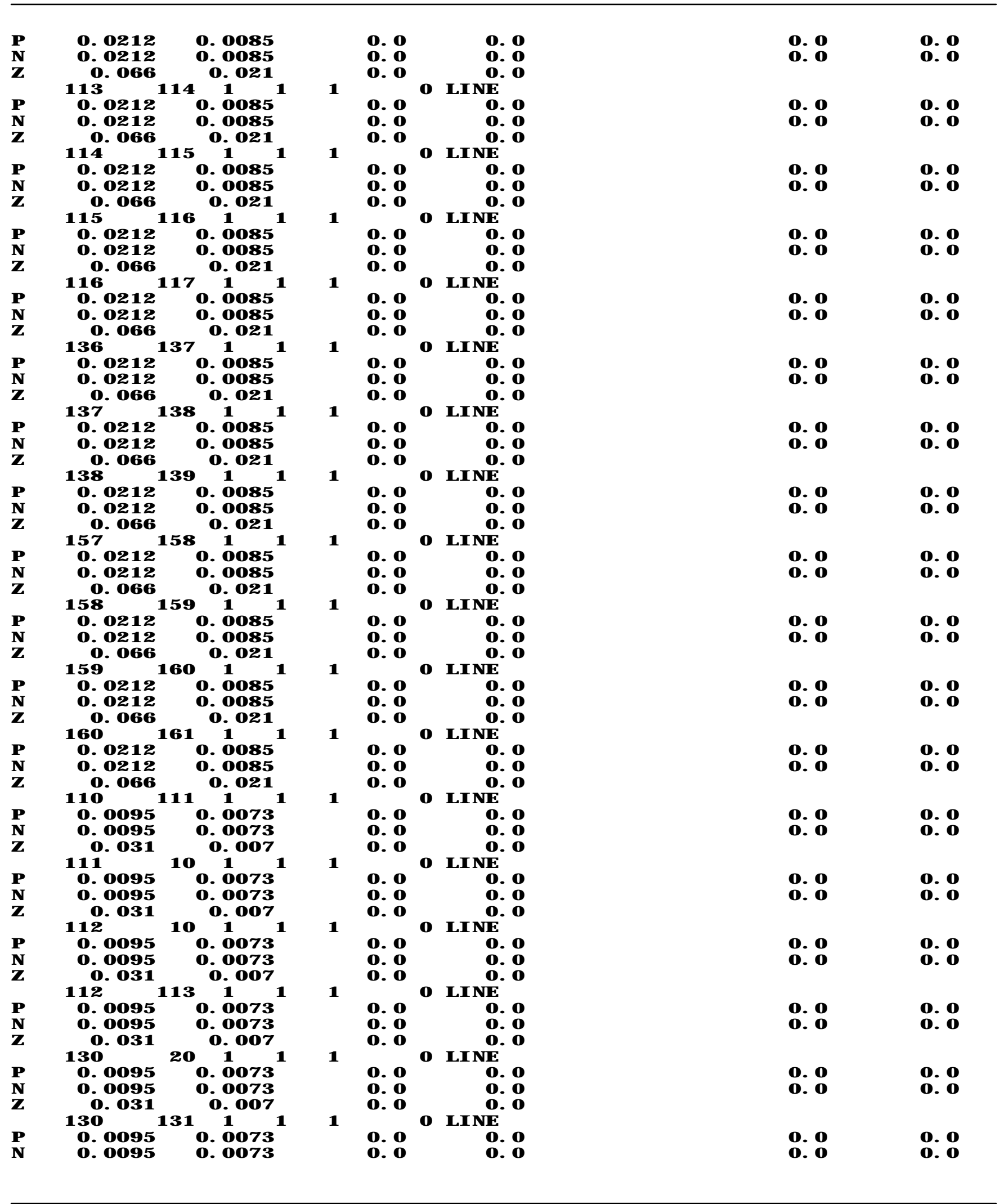




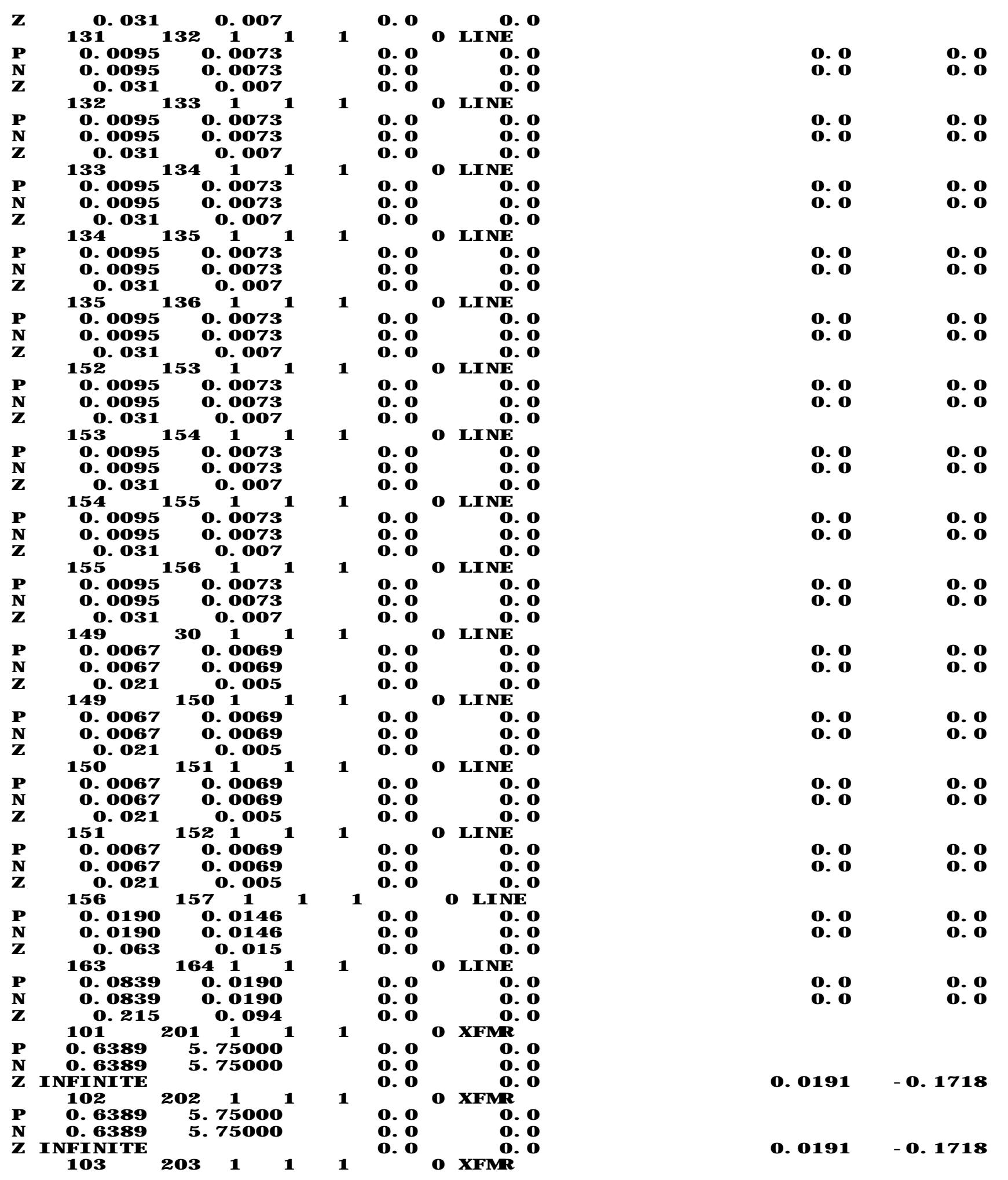




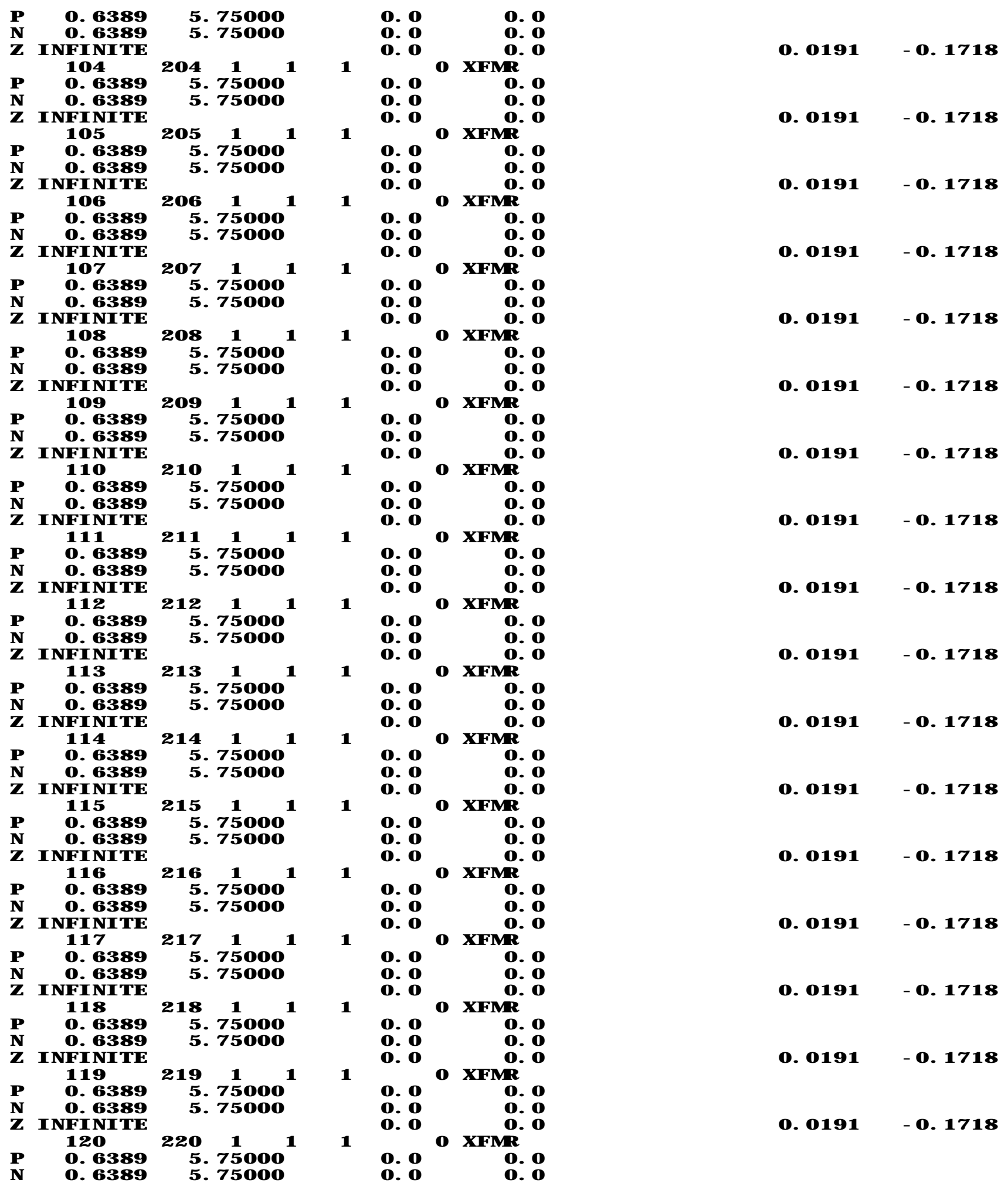




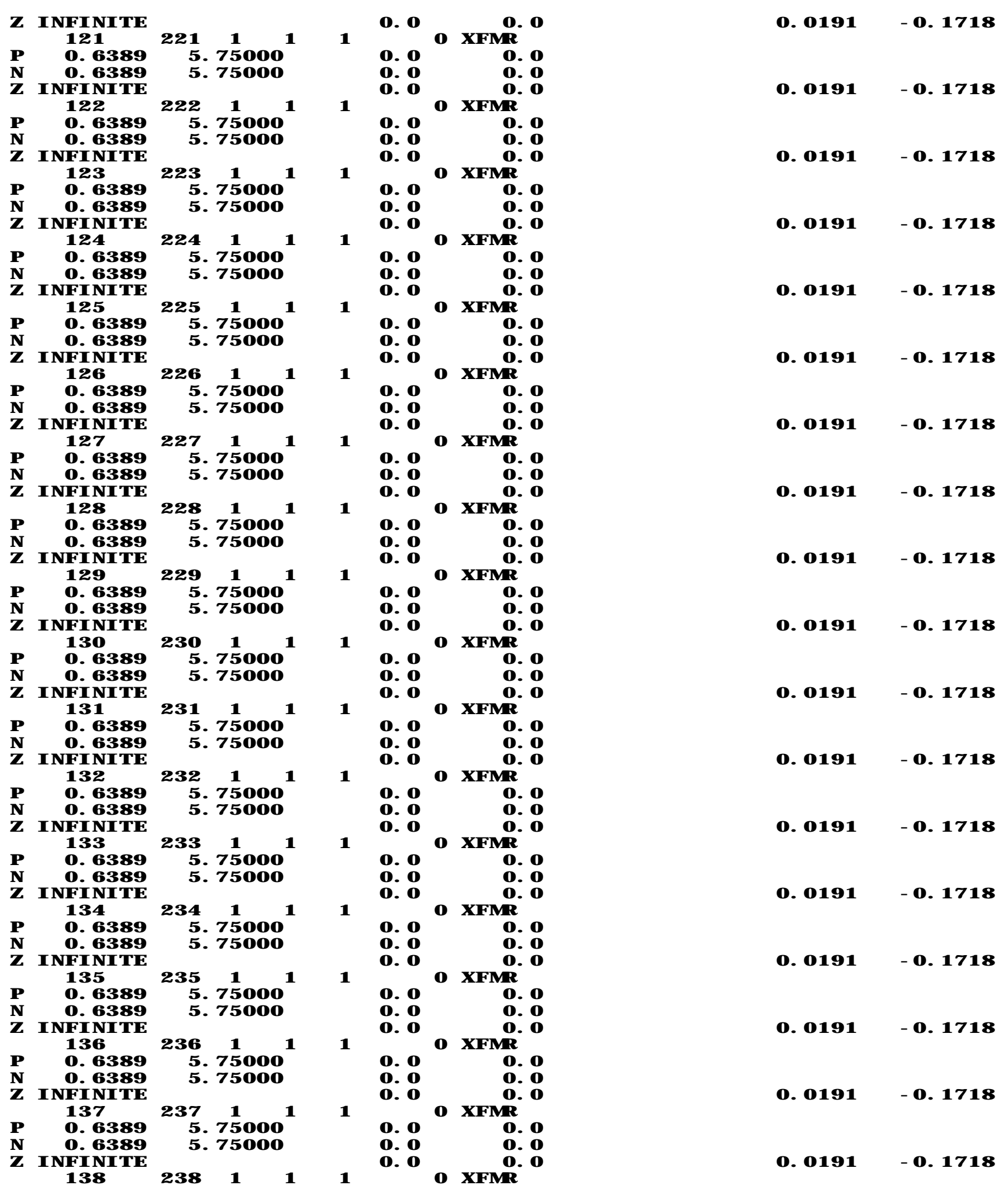




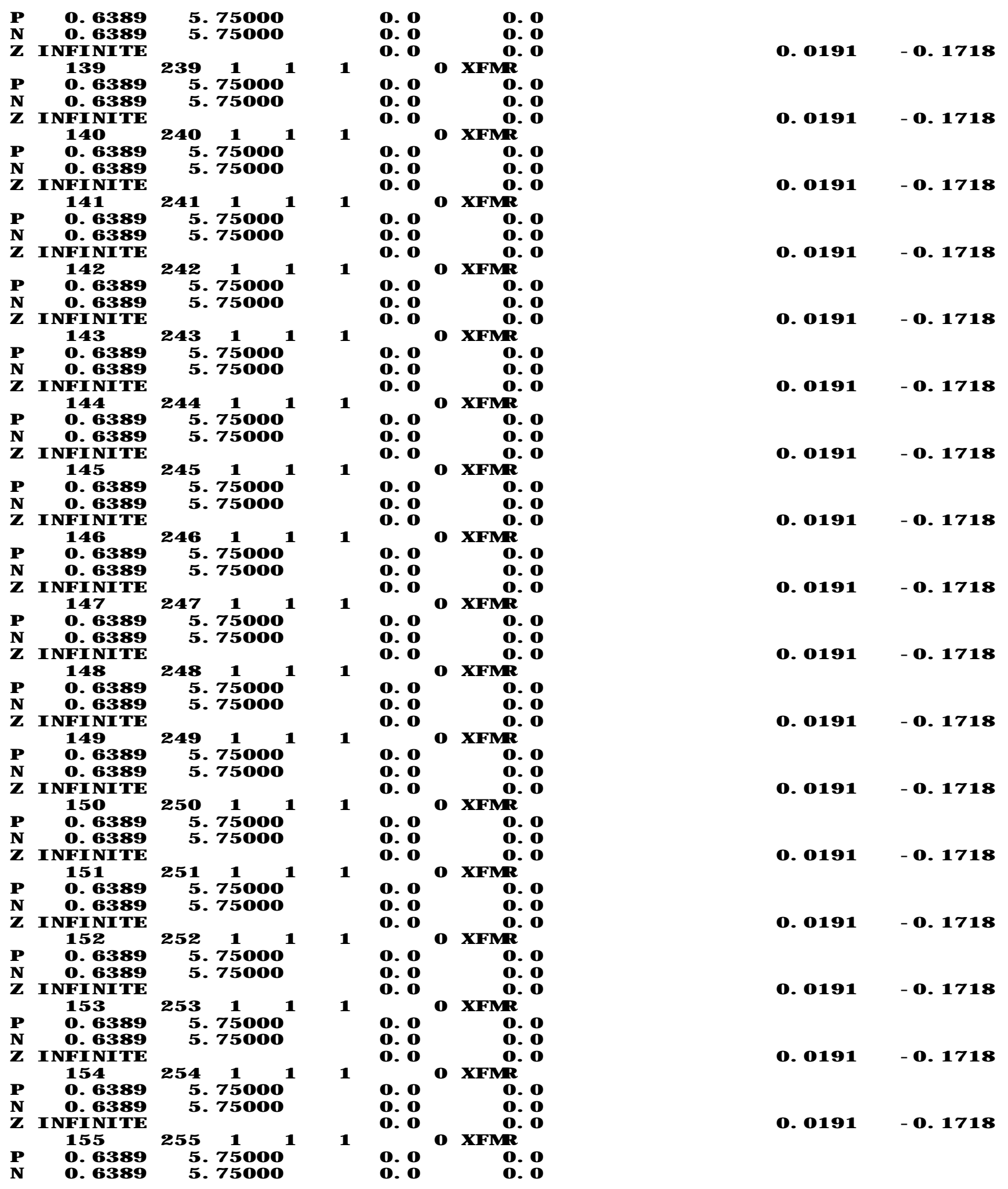




\begin{tabular}{|c|c|c|c|c|c|c|}
\hline Z I NFI NI TE & & & 0.0 & 0.0 & 0. 0191 & - 0.171 \\
\hline $\begin{array}{ll}P & 156 \\
0.6389\end{array}$ & ${ }^{256}{ }^{1} 7^{1}{ }^{1}{ }^{1}$ & 1 & $00^{c}$ & O XFMR & & \\
\hline$N \quad 0.6389$ & 5. 75000 & & 0.0 & O. 0 & & \\
\hline $\begin{array}{c}Z \text { I NFI NI TE } \\
157\end{array}$ & $257 \quad 1 \quad 1$ & 1 & 0.0 & O XFMR 0 & 0.0191 & - 0.171 \\
\hline 0. 6389 & 5. 75000 & + & o. 0 & O. 0 & & \\
\hline $\begin{array}{l}\mathrm{N} \\
\mathrm{Z}\end{array}$ & 5. 75000 & & $\begin{array}{l}\text { O. O } \\
\text { O. O }\end{array}$ & $\begin{array}{l}\text { O. O } \\
\text { O. O }\end{array}$ & 0. 0191 & -0.171 \\
\hline $\begin{array}{ll} & 158 \\
P & 0.6389\end{array}$ & 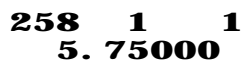 & 1 & o. o & $\begin{array}{l}\text { O XFMR } \\
\text { O. O }\end{array}$ & & \\
\hline $\begin{array}{l}\text { N O. } 6389 \\
Z \text { I NFI NII TE }\end{array}$ & 5. 75000 & & $\begin{array}{l}\text { O. } 0 \\
\text { O. } 0\end{array}$ & $\begin{array}{l}\text { O. } \mathrm{O} \\
\text { O. } \mathrm{O}\end{array}$ & 0. 0191 & -0.17 \\
\hline $\begin{array}{l}159 \\
P \quad 0389\end{array}$ & $259 \quad 11$ & 1 & & O XFMR & עצע & -0.18 \\
\hline $\begin{array}{l}\text { O. } 6389 \\
\text { O. } 6389\end{array}$ & $\begin{array}{l}\text { 5. } 75000 \\
5.75000\end{array}$ & & $\begin{array}{l}\text { O. } 0 \\
\text { O. } 0\end{array}$ & $\begin{array}{l}\text { O. O } \\
\text { O. O }\end{array}$ & & \\
\hline $\begin{array}{c}Z \quad I N F I N I T E \\
160\end{array}$ & 26011 & 1 & 0.0 & O XFMR & o. 0191 & -0.171 \\
\hline $\begin{array}{ll}\mathrm{P} & 0.6389 \\
\mathrm{~N} & \mathrm{O} \\
\mathrm{N}\end{array}$ & $\begin{array}{l}\text { 5. } 75000 \\
5.75000\end{array}$ & & $\begin{array}{l}\text { O. } \mathrm{O} \\
\text { O. O }\end{array}$ & $\begin{array}{l}\text { O. } \mathrm{O} \\
\text { O. } \mathrm{O}\end{array}$ & & \\
\hline $\begin{array}{c}Z \text { I NFI NI TE } \\
161\end{array}$ & 26111 & 1 & 0.0 & O XFMR & 0. 0191 & -0.17 \\
\hline $\begin{array}{ll}\mathrm{P} & 0.6389 \\
\mathrm{~N} & \mathrm{O} .6389\end{array}$ & $\begin{array}{l}5.75000 \\
5.75000\end{array}$ & & $\begin{array}{l}\text { O. O } \\
\text { O. O }\end{array}$ & $\begin{array}{l}\text { O. } \mathrm{O} \\
\text { O. O }\end{array}$ & & \\
\hline $\begin{array}{ll}Z & \text { I NFI NI TE } \\
\text { P } & 162 \\
\mathrm{~N} & 0.6389 \\
\mathrm{~N} & 0.6389\end{array}$ & $\begin{array}{c}262.1 \\
5.75000 \\
5.75000\end{array}$ & 1 & $\begin{array}{l}0.0 \\
\text { O. O } \\
\text { O. O }\end{array}$ & $\begin{array}{r}\text { O. O } \\
\text { O XFMR } \\
\text { O. O } \\
\text { O. O }\end{array}$ & 0. 0191 & -0.17 \\
\hline $\begin{array}{l}\mathrm{N} \text { O. } 6389 \\
\mathrm{Z} \text { I NFI NI TE }\end{array}$ & 5.75000 & & $\begin{array}{l}\text { O. O } \\
\text { O. O }\end{array}$ & $\begin{array}{l}\text { O. O } \\
\text { O. } \mathrm{O}\end{array}$ & 0. 0191 & -0.17 \\
\hline $\begin{array}{ll} & 163 \\
\mathrm{P} & 0.6389 \\
\mathrm{~N} & 0.6389\end{array}$ & $\begin{array}{c}263.1 \\
5.75000 \\
5.75000\end{array}$ & 1 & $\begin{array}{l}\text { O. o } \\
\text { O. O }\end{array}$ & $\begin{array}{r}\text { O XFMR } \\
\text { O. O } \\
\text { O. O }\end{array}$ & & \\
\hline $\begin{array}{c}Z \text { I NFI NI TE } \\
164\end{array}$ & & & 0.0 & O. 0 & o. 0191 & -0.171 \\
\hline $\begin{array}{ll}\mathrm{P} & 164 \\
\mathrm{~N} & \mathrm{O} \\
\mathrm{O} & \mathrm{O} .63899 \\
\end{array}$ & $\begin{array}{l}264.1 \\
5.75000 \\
5.75000\end{array}$ & 1 & $\begin{array}{l}\text { O. O } \\
\text { O. O }\end{array}$ & $\begin{array}{r}0 \text { XFMR } \\
\text { O. O } \\
\text { O. O }\end{array}$ & & \\
\hline $\begin{array}{l}\text { Z I NFI NI TE } \\
165\end{array}$ & $26511 \quad 1$ & 1 & 0.0 & O XFMR 0 & 0. 0191 & -0.17 \\
\hline $\begin{array}{lr}P & 0.6389 \\
N & O .6389 \\
Z & \text { I NFi NI TE }\end{array}$ & $\begin{array}{l}\text { 5. } 75000 \\
\text { 5. } 75000\end{array}$ & & $\begin{array}{l}\text { O. O } \\
\text { O. O } \\
\text { O. O }\end{array}$ & $\begin{array}{l}\text { O. O } \\
\text { O. O } \\
\text { O. O }\end{array}$ & 0. 0191 & - 0.17 \\
\hline $\begin{array}{lr} & 166 \\
P & 0.6389 \\
N & O .6389 \\
Z & \text { I NFI NI TE }\end{array}$ & $\begin{array}{c}266.7 \\
5.75000 \\
5.75000\end{array}$ & 1 & $\begin{array}{l}\text { O. O } \\
\text { O. O } \\
\text { O. O }\end{array}$ & $\begin{array}{r}\text { O XFMR } \\
\text { O. O } \\
\text { O. O } \\
\text { O. O }\end{array}$ & 0. 0191 & $-0.17]$ \\
\hline
\end{tabular}

MUTUAL DATA FOLLONS

END 


\section{Table E1 Summary}

Table E2.1A 750 kW - 15-kV Plan Cost Summary

Table E2.1B 750 kW - 25-kV Plan Cost Summary

Table E2.2A 2,5000 kW - 25-kV Plan Cost Summary

Table E2.2B 2,500 kW - 35-kV Plan Cost Summary

Table E2.3A 5,000 kW - 25-kV Plan Cost Summary

Table E2.3B 5,000 kW - 35-kV Plan Cost Summary

Table E2.4A 10,000 kW - 25-kV Plan Cost Summary

Table E2.4B 10,000 kW - 35-kV Plan Cost Summary

Table E3.1A 750 kW - 15-kV Plan Padmount Transformer Cost Detail

Table E3.1B 750 kW - 25-kV Plan Padmount Transformer Cost Detail

Table E3.2A 2500 kW - 25-kV Plan Padmount Transformer Cost Detail

Table E3.2B 2500 kW - 35-kV Plan Padmount Transformer Cost Detail

Table E3.3A 5000 kW - 25-kV Plan Padmount Transformer Cost Detail

Table E3.3B 5000 kW - 35-kV Plan Padmount Transformer Cost Detail

Table E3.4A 10000 kW - 25-kV Plan Padmount Transformer Cost Detail

Table E3.4B 10000 kW - 35-kV Plan Padmount Transformer Cost Detail

Table E4.1A 750 kW - 15-kV Plan Underground Cable Cost Detail

Table E4.1B $750 \mathrm{~kW}$ - 25-kV Plan Underground Cable Cost Detail

Table E4.2A $2500 \mathrm{~kW}$ - 25-kV Plan Underground Cable Cost Detail

Table E4.2B 2500 kW - 35-kV Plan Underground Cable Cost Detail

Table E4.3A 5000 kW - 25-kV Plan Underground Cable Cost Detail

Table E4.3B 5000 kW - 35-kV Plan Underground Cable Cost Detail

Table E4.4A 10000 kW - 25-kV Plan Underground Cable Cost Detail

Table E4.4B 10000 kW - 35-kV Plan Underground Cable Cost Detail

Table E5.1A 750 kW - 15-kV Plan Recloser and Riser Cost Detail Table E5.1B $750 \mathrm{~kW}$ - 25-kV Plan Recloser and Riser Cost Detail Table E5.2A 2500 kW - 25-kV Plan Recloser and Riser Cost Detail Table E5.2B 2500 kW - 35-kV Plan Recloser and Riser Cost Detail Table E5.3A 5000 kW - 25-kV Plan Recloser and Riser Cost Detail Table E5.3B 5000 kW - 35-kV Plan Recloser and Riser Cost Detail Table E5.4A $10000 \mathrm{~kW}$ - 25-kV Plan Recloser and Riser Cost Detail Table E5.4B 10000 kW - 35-kV Plan Recloser and Riser Cost Detail 
Table E6.1A 750 kW - 15-kV Plan Overhead Line Cost Detail

Table E6.1B 750 kW - 25-kV Plan Overhead Line Cost Detail

Table E6.2A 2500 kW - 25-kV Plan Overhead Line Cost Detail

Table E6.2B 2500 kW - 35-kV Plan Overhead Line Cost Detail

Table E6.3A 5000 kW - 25-kV Plan Overhead Line Cost Detail

Table E6.3B 5000 kW - 35-kV Plan Overhead Line Cost Detail

Table E6.4A 10000 kW - 25-kV Plan Overhead Line Cost Detail

Table E6.4B 10000 kW - 35-kV Plan Overhead Line Cost Detail

Table E7.1 New 15-kV PDS Substation Installed Adjacent to Existing 115-kV Line Table E7.2 New 25-kV PDS Substation Installed Adjacent to Existing 115-kV Line

Table E7.3 New 35-kV PDS Substation Installed Adjacent to Existing 115-kV Line

Table E8.1 20' Stone Service Road for 750-kW Plan

Table E8.2 20' Stone Service Road for 2,500-kW Plan

Table E8.3 20' Stone Service Road for 5,000-kW Plan

Table E8.4 20' Stone Service Road for 10,000-kW Plan

Table E8.1a 26' Stone Service Road for 750-kW Plan

Table E8.2a 26' Stone Service Road for 2,500-kW Plan

Table E8.3a 26' Stone Service Road for 5,000-kW Plan

Table E8.4a 26' Stone Service Road for 10,000-kW Plan

Table E8.1b 30' Stone Service Road for 750-kW Plan

Table E8.2b 30' Stone Service Road for 2,500-kW Plan

Table E8.3b 40' Stone Service Road for 5,000-kW Plan

Table E8.4b 40' Stone Service Road for 10,000-kW Plan

Table E9 Maintenance Building All Plans

Table E10 Communications Cost Detail

Table E10.1 750-kW Communications Cost Detail

Table E10.2 2,500-kW Communications Cost Detail

Table E10.3 5,000-kW Communications Cost Detail

Table E11 Crane Pad Cost Detail

Table E12 Vendor Quote for the Major Electrical Equipment 


\section{APPENDIX E COST ESTIMATES}

Table E1 provides a summary of the balance-of-station cost for all plans studied. Tables E2.1A through E2.4B provide the detailed estimate for each plan. The cost estimates are subtotaled by electrical, civil, communications, miscellaneous infrastructure, land, clearing, and project overhead costs. Also, average unit costs are provided where the cost can be related to either a number or length. For example, a generator step-up transformer is required for each wind turbine, so the unit cost for generator step-up transformers relates to the number of wind turbines installed. Cable and road unit costs are related to the length of these items. All costs are provided in present-day dollars.

CAI developed the cost estimates using internal data, manufacturers' equipment quotes, MEANS 2000 Electrical Cost Data, and MEANS 2000 Building Construction Cost Data. Details are provided in Tables E3 through E11. Table E12 summarizes manufacturer cost data for the major electrical equipment.

Additional assumptions are listed below:

1. The cost estimates do not include a low-voltage main circuit breaker on the secondary of the generator step-up transformers. It is assumed that this breaker is part of the wind turbine electrical system. Some coordination will be required to ensure that this main breaker can be tripped for a transformer fault. It might be necessary to add more relaying to the transformer to ensure this protection is adequately designed.

2. The underground costs are based on direct-buried cable installed in a trench. The trenching costs were taken from MEANS. This cost is based on trench in standard earth. We also assumed that any excess soil would be disposed of on-site at no cost. If there is considerable rock, then the trenching cost should be increased, perhaps doubled. Also, it may be necessary to haul and dispose of rocks that are brought to the surface. The cost for this would need to be added to the estimate.

3. No costs for line clearing were included. It is assumed that no clearing will be necessary at the Mission site.

4. Costs for land and right-of-way were included for the substation, maintenance building, and overhead line only. It is assumed that costs for the land rights for the underground cable and the access roadways will be negotiated as part of the land rights negotiated for the wind turbines.

5. The substation cost is based on installing a Power Delivery System (PDS) at a new substation site, as described in Appendix A. Rather than using this packaged system as assumed, a lower-cost substation may be possible by purchasing a stand-alone transformer and distribution breakers. This would be especially true if the existing 
substation site is already developed for a second transformer and if the utility would allow for the wind farm transformer to be located at that site.

6. The substation cost estimates include a cost for a power electronic switched capacitor that would provide for delivery of 0 to $50 \mathrm{MW}$ at a near-unity power factor.

7. Three options were considered for the access roads. The access roads are located only between wind turbines. Option 1 is based on a minimal 20 -foot-wide gravel road. Option 2 is based on a paved 26-foot-wide road for the 750 - and 2,500-kW plans and 32-footwide roads for the 5-MW and 10-MW plans. Option 2 road widths provide enough space to move a maintenance crane between towers but would not be wide enough to move the construction crane from site to site without disassembly. Option 3 road widths are 30 feet for the 750- and 2,500-kW plans and 40 feet for the 5-MW and 10-MW plans. These road widths would allow the construction crane to move from site to site. The gentleness of the Mission site terrain minimized the need for cut-and-fill of the roadbed. A steeper terrain would increase the road costs.

8. We have not included any costs to upgrade county or township roads. 
TABLE E1

WindPACT Turbine Design Scaling Study

Technical Area 4 Balance of Station Cost

Cost Estimate (\$ X 1,000)

SUMMARY SHEET

\begin{tabular}{|c|c|c|c|c|c|c|c|c|}
\hline \multirow{2}{*}{ System Component } & \multicolumn{2}{|c|}{750 kW Plan } & \multicolumn{2}{|c|}{2500 kW Plan } & \multicolumn{2}{|c|}{5000 kW Plan } & \multicolumn{2}{|c|}{10000 kW Plan } \\
\hline & $15 \mathrm{kV}$ & $25 \mathrm{kV}$ & $25 \mathrm{kV}$ & $35 \mathrm{kV}$ & $25 \mathrm{kV}$ & $35 \mathrm{kV}$ & $25 \mathrm{kV}$ & $35 \mathrm{kV}$ \\
\hline Generator Step-up Transformers & 1,147 & 1,153 & 676 & 710 & 1,177 & 1,196 & 819 & 870 \\
\hline Underground Cable & 643 & 610 & 334 & 356 & 221 & 237 & 154 & 174 \\
\hline Risers and Reclosers & 126 & 129 & 103 & 83 & 77 & 83 & 77 & 83 \\
\hline Overhead Line & 188 & 123 & 121 & 121 & 333 & 301 & 98 & 98 \\
\hline Substation & 2,612 & 2,528 & 2,528 & 2,729 & 2,528 & 2,729 & 2,528 & 2,729 \\
\hline Subtotal Electrical & 4,716 & 4,543 & 3,762 & 3,999 & 4,337 & 4,545 & 3,677 & 3,954 \\
\hline Generator Foundations & 5,148 & 5,148 & 7,000 & 7,000 & 8,300 & 8,300 & 8,000 & 8,000 \\
\hline Service Roads (Option 2) & 2,310 & 2,310 & 1,156 & 1,156 & 1,000 & 1,000 & 680 & 680 \\
\hline Crane Pads & 1,386 & 1,386 & 1,200 & 1,200 & 1,900 & 1,900 & 1,585 & 1,585 \\
\hline Subtotal Civil & 8,844 & 8,844 & 9,356 & 9,356 & 11,200 & 11,200 & 10,265 & 10,265 \\
\hline Communications & 823 & 823 & 380 & 372 & 335 & 335 & 209 & 209 \\
\hline Tower Lighting & 660 & 660 & 200 & 200 & 100 & 100 & 50 & 50 \\
\hline Meteorological Towers & 74 & 74 & 88 & 88 & 106 & 106 & 160 & 160 \\
\hline Maintenance Building & 156 & 156 & 156 & 156 & 156 & 156 & 156 & 156 \\
\hline Subtotal Misc. Infrastructure & 1,713 & 1,713 & 824 & 816 & 697 & 697 & 575 & 575 \\
\hline Land and Right-of-Way & 47 & 47 & 47 & 47 & 76 & 76 & 44 & 44 \\
\hline Clearing and Site Preparation & 5 & 5 & 5 & 5 & 5 & 5 & 5 & 5 \\
\hline Project Overheads & 1,280 & 1,268 & 1,161 & 1,177 & 1,334 & 1,348 & 1,186 & 1,205 \\
\hline Subtotal (RW, Clearing \& Overhead) & 1,332 & 1,319 & 1,213 & 1,229 & 1,414 & 1,429 & 1,234 & 1,254 \\
\hline Project Subtotal & 16,604 & 16,419 & 15,155 & 15,400 & 17,648 & 17,872 & 15,751 & 16,048 \\
\hline Contingency & 3,321 & 3,284 & 3,031 & 3,080 & 3,530 & 3,574 & 3,150 & 3,210 \\
\hline Plan Totals: & 19,920 & 19,700 & 18,190 & 18,480 & 21,180 & 21,450 & 18,900 & 19,260 \\
\hline
\end{tabular}


Table E2.1A

750kW - 15 kV Plan Cost Summary

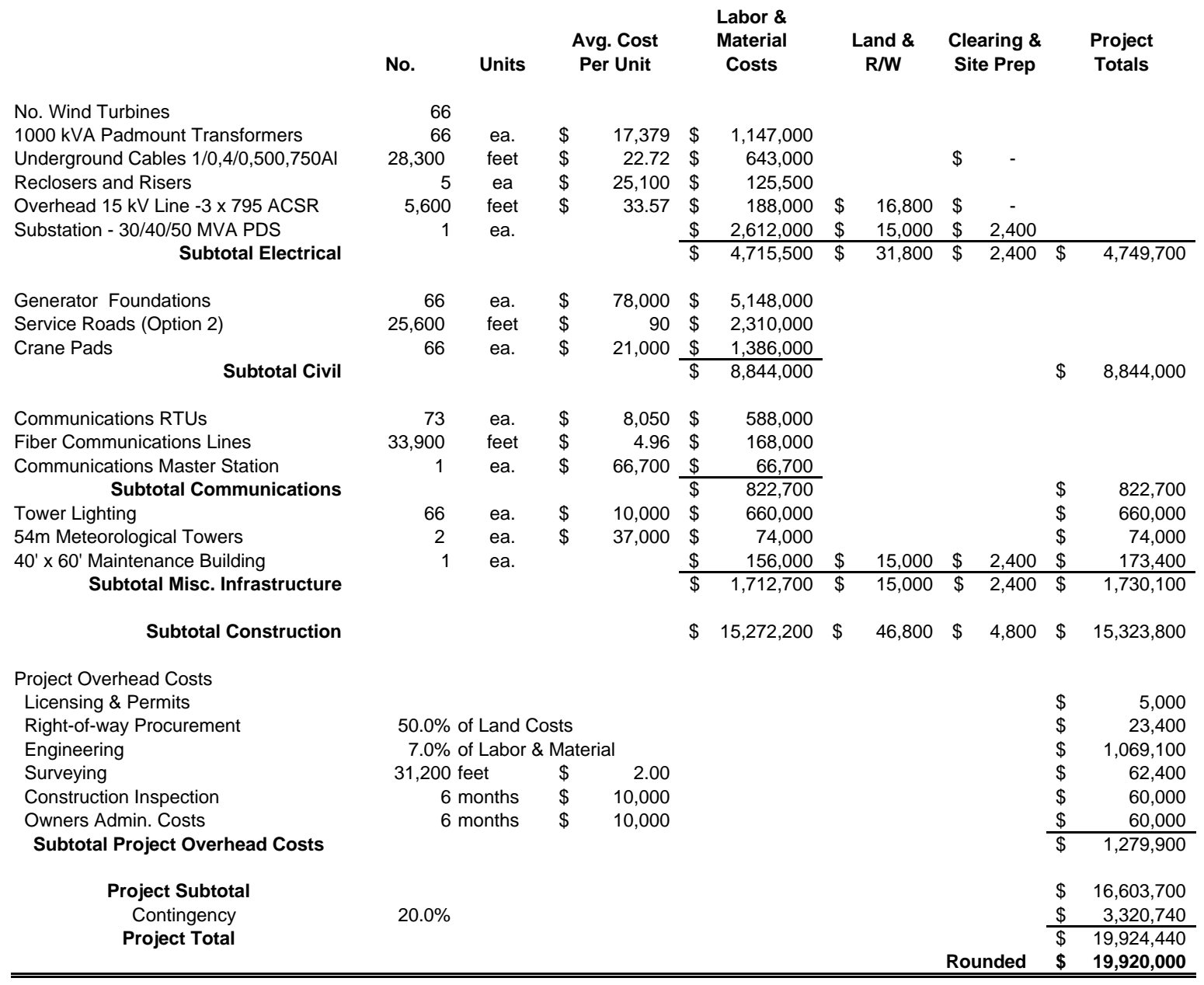


Table E2.1B

750kW - 25 kV Plan Cost Summary

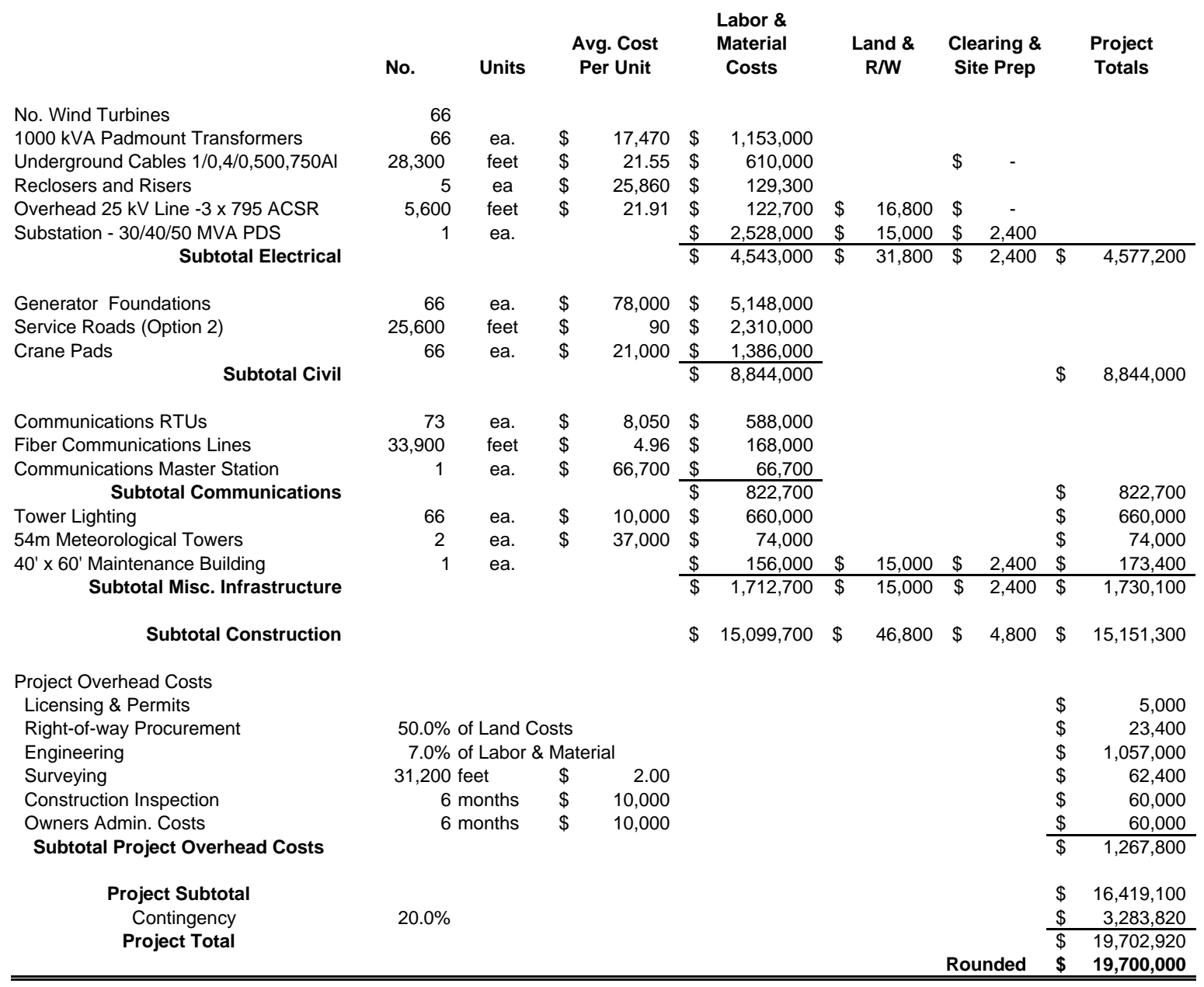


Table E2.2A

\section{0kW - 25 kV Plan Cost Summary}

\begin{tabular}{|c|c|c|c|c|c|c|c|c|c|c|c|c|}
\hline \multirow[b]{2}{*}{ No. Wind Turbines } & No. & \multirow[t]{2}{*}{ Units } & \multicolumn{2}{|c|}{$\begin{array}{l}\text { Avg. Cost } \\
\text { Per Unit }\end{array}$} & \multicolumn{2}{|r|}{$\begin{array}{c}\text { Labor \& } \\
\text { Material } \\
\text { Costs }\end{array}$} & \multicolumn{2}{|r|}{$\begin{array}{c}\text { Land \& } \\
\text { R/W }\end{array}$} & \multicolumn{3}{|c|}{$\begin{array}{l}\text { Clearing \& } \\
\text { Site Prep }\end{array}$} & \multirow[t]{2}{*}{$\begin{array}{l}\text { Project } \\
\text { Totals }\end{array}$} \\
\hline & 20 & & & & & & & & & & & \\
\hline 3000 kVA Padmount Transformers & 20 & ea. & $\$$ & 33,800 & $\$$ & 676,000 & & & & & & \\
\hline Underground Cables $1 / 0,4 / 0,500,750 \mathrm{Al}$ & 17,300 & feet & $\$$ & 19.31 & $\$$ & 334,000 & & & $\$$ & - & & \\
\hline Reclosers and Risers & 4 & ea & $\$$ & 25,650 & $\$$ & 102,600 & & & & & & \\
\hline Overhead 25 kV Line $-3 \times 795$ ACSR & 5,700 & feet & $\$$ & 21.26 & $\$$ & 121,200 & $\$$ & 17,100 & $\$$ & - & & \\
\hline Substation - 30/40/50 MVA PDS & 1 & ea. & & & $\$$ & $2,528,000$ & $\$$ & 15,000 & $\$$ & 2,400 & & \\
\hline Subtotal Electrical & & & & & $\$$ & $3,761,800$ & $\$$ & 32,100 & $\$$ & 2,400 & $\$$ & $3,796,300$ \\
\hline Generator Foundations & 20 & ea. & $\$$ & 350,000 & $\$$ & $7,000,000$ & & & & & & \\
\hline Service Roads (Option 2) & 12,800 & feet & $\$$ & 90 & $\$$ & $1,156,000$ & & & & & & \\
\hline Crane Pads & 20 & ea. & $\$$ & 60,000 & $\$$ & $1,200,000$ & & & & & & \\
\hline Subtotal Civil & & & & & $\$$ & $9,356,000$ & & & & & $\$$ & $9,356,000$ \\
\hline Communications RTUs & 26 & ea. & $\$$ & 8,050 & $\$$ & 209,300 & & & & & & \\
\hline Fiber Communications Lines & 19,600 & feet & $\$$ & 5.31 & $\$$ & 104,000 & & & & & & \\
\hline Communications Master Station & 1 & ea. & $\$$ & 66,700 & $\$$ & 66,700 & & & & & & \\
\hline Subtotal Communications & & & & & $\$$ & 380,000 & & & & & $\$$ & 380,000 \\
\hline Tower Lighting & 20 & ea. & $\$$ & 10,000 & $\$$ & 200,000 & & & & & $\$$ & 200,000 \\
\hline 92m Meteorological Towers & 2 & ea. & $\$$ & 44,000 & $\$$ & 88,000 & & & & & $\$$ & 88,000 \\
\hline $40^{\prime} \times 60^{\prime}$ Maintenance Building & 1 & ea. & & & $\$$ & 156,000 & $\$$ & 15,000 & $\$$ & 2,400 & $\$$ & 173,400 \\
\hline Subtotal Misc. Infrastructure & & & & & $\$$ & 824,000 & $\$$ & 15,000 & $\$$ & 2,400 & $\$$ & 841,400 \\
\hline Subtotal Construction & & & & & $\$$ & $13,941,800$ & $\$$ & 47,100 & $\$$ & 4,800 & $\$$ & $13,993,700$ \\
\hline \multicolumn{13}{|l|}{ Project Overhead Costs } \\
\hline Licensing \& Permits & \multirow{2}{*}{\multicolumn{4}{|c|}{$50.0 \%$ of Land Costs }} & & & & & & & $\$$ & 5,000 \\
\hline Right-of-way Procurement & & & & & & & & & & & $\$$ & 23,400 \\
\hline Engineering & \multicolumn{4}{|c|}{$7.0 \%$ of Labor \& Material } & & & & & & & $\$$ & 975,900 \\
\hline Surveying & 18,500 & & $\$$ & 2.00 & & & & & & & $\$$ & 37,000 \\
\hline Construction Inspection & & onths & $\$$ & 10,000 & & & & & & & $\$$ & 60,000 \\
\hline Owners Admin. Costs & & onths & $\$$ & 10,000 & & & & & & & $\$$ & 60,000 \\
\hline Subtotal Project Overhead Costs & & & & & & & & & & & $\$$ & $1,161,300$ \\
\hline Project Subtotal & & & & & & & & & & & $\$$ & $15,155,000$ \\
\hline Contingency & $20.0 \%$ & & & & & & & & & & $\$$ & $3,031,000$ \\
\hline \multirow[t]{2}{*}{ Project Total } & & & & & & & & & & & $\$$ & $18,186,000$ \\
\hline & & & & & & & & & \multicolumn{2}{|c|}{ Rounded } & $\$$ & $18,190,000$ \\
\hline
\end{tabular}


Table E2.2B

2500kW - 35 kV Plan Cost Summary

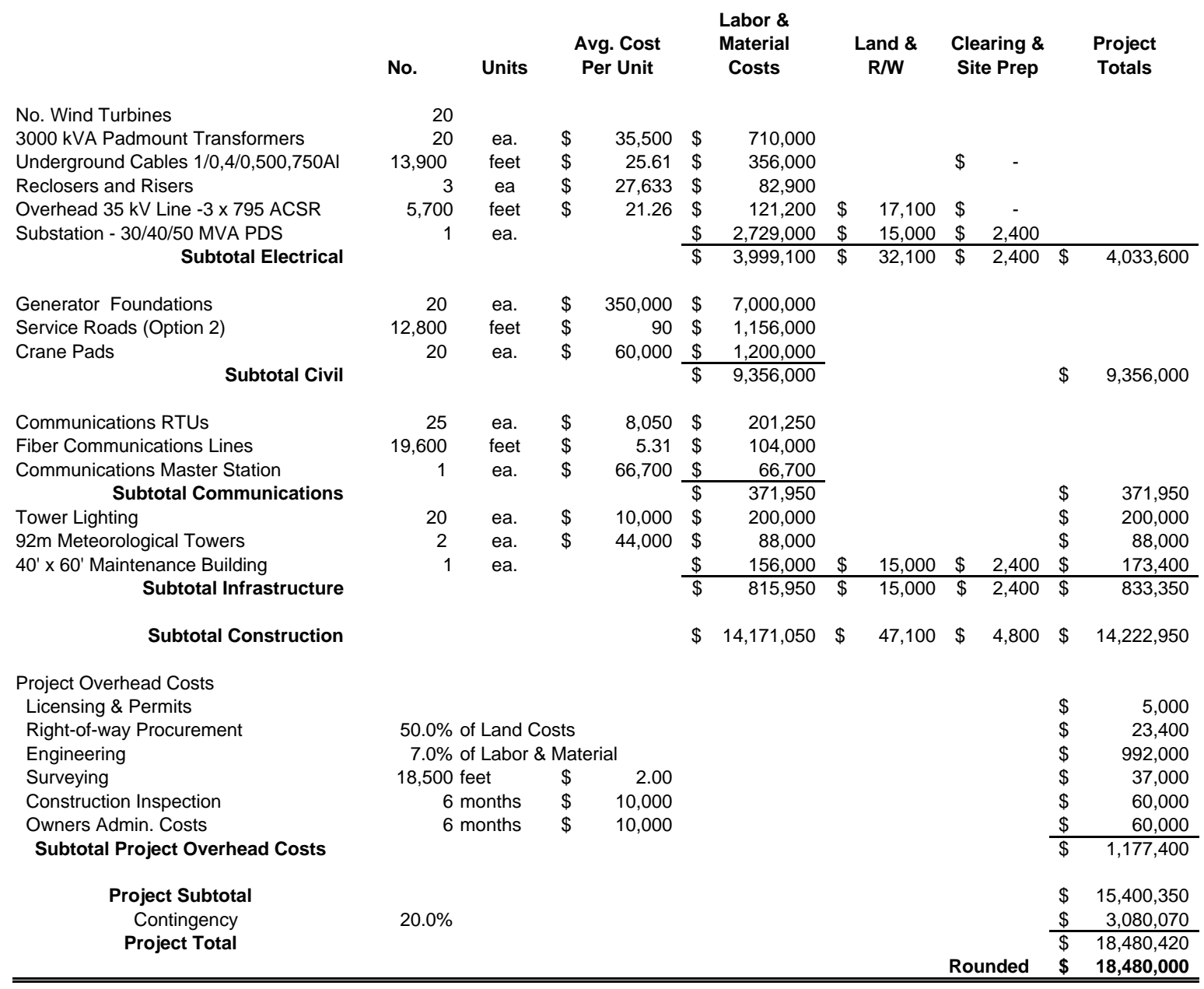


Table E2.3A

5000 kW - 25 kV Plan Cost Summary

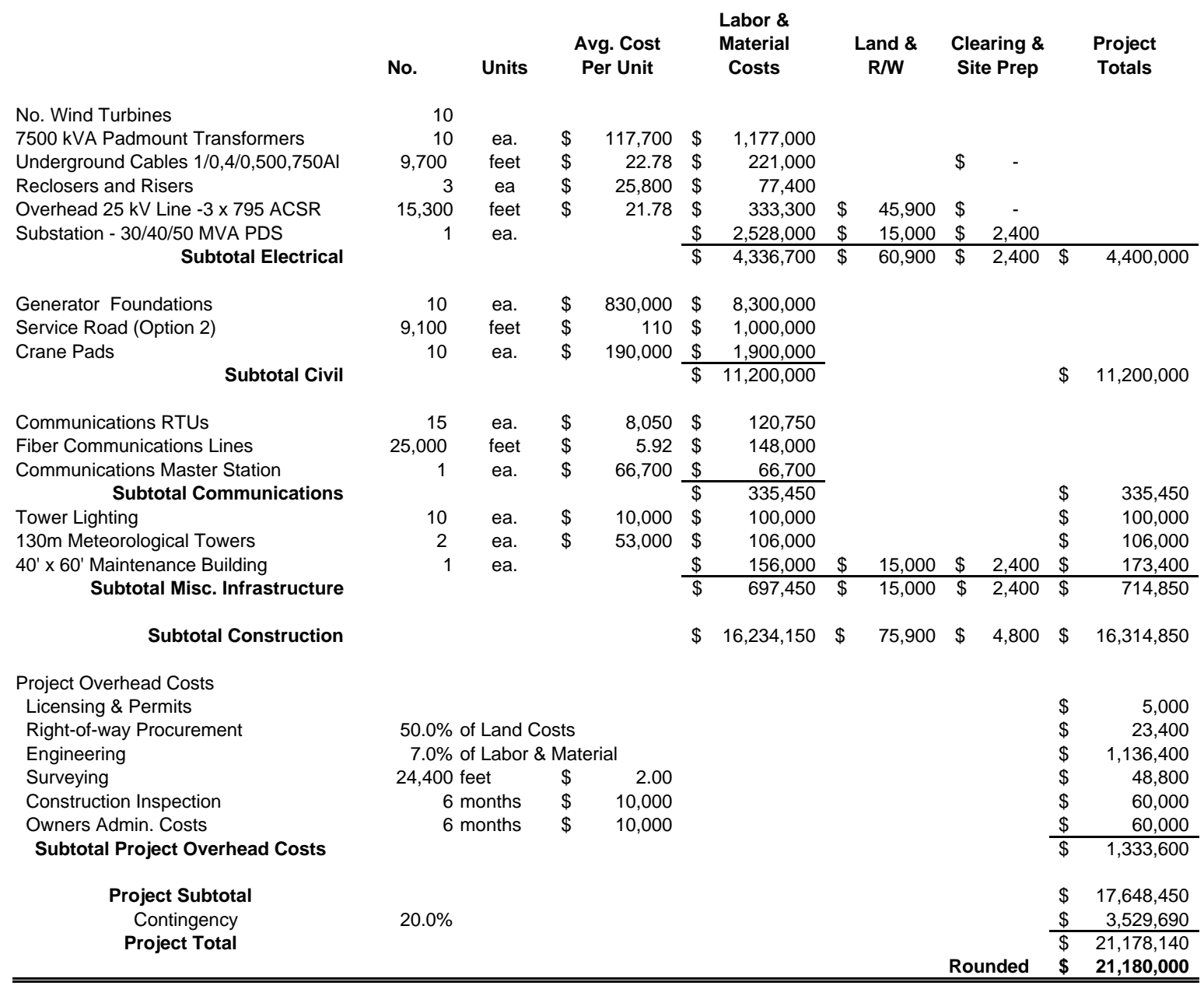


Table E2.3B

$5000 k W$ - 35 kV Plan Cost Summary

\begin{tabular}{|c|c|c|c|c|c|c|c|c|c|c|c|c|}
\hline \multirow[b]{2}{*}{ No. Wind Turbines } & No. & \multirow[t]{2}{*}{ Units } & \multicolumn{2}{|c|}{$\begin{array}{l}\text { Avg. Cost } \\
\text { Per Unit }\end{array}$} & \multicolumn{2}{|r|}{$\begin{array}{c}\text { Labor \& } \\
\text { Material } \\
\text { Costs }\end{array}$} & \multicolumn{2}{|r|}{$\begin{array}{l}\text { Land \& } \\
\mathrm{R} / \mathrm{W}\end{array}$} & \multicolumn{2}{|c|}{$\begin{array}{l}\text { Clearing \& } \\
\text { Site Prep }\end{array}$} & & \multirow[t]{2}{*}{$\begin{array}{c}\text { Project } \\
\text { Totals }\end{array}$} \\
\hline & 10 & & & & & & & & & & & \\
\hline 7500 kVA Padmount Transformers & 10 & ea. & $\$$ & 119,600 & $\$$ & $1,196,000$ & & & & & & \\
\hline Underground Cables 1/0,4/0,500,750AI & 9,700 & feet & $\$$ & 24.43 & $\$$ & 237,000 & & & $\$$ & - & & \\
\hline Reclosers and Risers & 3 & ea & $\$$ & 27,600 & $\$$ & 82,800 & & & & & & \\
\hline Overhead 35 kV Line $-3 \times 477$ ACSR & 15,300 & feet & $\$$ & 19.65 & $\$$ & 300,600 & $\$$ & 45,900 & $\$$ & - & & \\
\hline Substation - 30/40/50 MVA PDS & 1 & ea. & & & $\$$ & $2,729,000$ & $\$$ & 15,000 & $\$$ & 2,400 & & \\
\hline \multicolumn{2}{|l|}{ Subtotal Electrical } & & & & $\$$ & $4,545,400$ & $\$$ & 60,900 & $\$$ & 2,400 & $\$$ & $4,608,700$ \\
\hline Generator Foundations & 10 & ea. & $\$$ & 830,000 & $\$$ & $8,300,000$ & & & & & & \\
\hline 20' Service Roads & 9,060 & feet & $\$$ & 110 & $\$$ & $1,000,000$ & & & & & & \\
\hline Crane Pads & 10 & ea. & $\$$ & 190,000 & $\$$ & $1,900,000$ & & & & & & \\
\hline Subtotal Civil & & & & & $\$$ & $11,200,000$ & & & & & $\$$ & $11,200,000$ \\
\hline Communications RTUs & 15 & ea. & $\$$ & 8,050 & $\$$ & 120,750 & & & & & & \\
\hline Fiber Communications Lines & 25,000 & feet & $\$$ & 5.92 & $\$$ & 148,000 & & & & & & \\
\hline Communications Master Station & 1 & ea. & $\$$ & 66,700 & $\$$ & 66,700 & & & & & & \\
\hline Subtotal Communications & & & & & $\$$ & 335,450 & & & & & $\$$ & 335,450 \\
\hline Tower Lighting & 10 & ea. & $\$$ & 10,000 & $\$$ & 100,000 & & & & & $\$$ & 100,000 \\
\hline 130m Meteorological Towers & 2 & ea. & $\$$ & 53,000 & $\$$ & 106,000 & & & & & $\$$ & 106,000 \\
\hline 40' x 60' Maintenance Building & 1 & ea. & & & $\$$ & 156,000 & $\$$ & 15,000 & $\$$ & 2,400 & $\$$ & 173,400 \\
\hline Subtotal Misc. Infrastructure & & & & & $\$$ & 697,450 & $\$$ & 15,000 & $\$$ & 2,400 & $\$$ & 714,850 \\
\hline Subtotal Construction & & & & & $\$$ & $16,442,850$ & $\$$ & 75,900 & $\$$ & 4,800 & $\$$ & $16,523,550$ \\
\hline \multicolumn{13}{|l|}{ Project Overhead Costs } \\
\hline Licensing \& Permits & & & & & & & & & & & $\$$ & 5,000 \\
\hline Right-of-way Procurement & \multicolumn{4}{|c|}{$50.0 \%$ of Land Costs } & & & & & & & $\$$ & 23,400 \\
\hline Engineering & \multicolumn{4}{|c|}{$7.0 \%$ of Labor \& Material } & & & & & & & $\$$ & $1,151,000$ \\
\hline Surveying & 24,360 & & $\$$ & 2.00 & & & & & & & $\$$ & 48,720 \\
\hline Construction Inspection & & onths & $\$$ & 10,000 & & & & & & & $\$$ & 60,000 \\
\hline Owners Admin. Costs & & onths & $\$$ & 10,000 & & & & & & & $\$$ & 60,000 \\
\hline Subtotal Project Overhead Costs & & & & & & & & & & & $\$$ & $1,348,120$ \\
\hline Project Subtotal & & & & & & & & & & & $\$$ & $17,871,670$ \\
\hline Contingency & $20.0 \%$ & & & & & & & & & & $\$$ & $3,574,334$ \\
\hline \multirow[t]{2}{*}{ Project Total } & & & & & & & & & & & $\$$ & $21,446,004$ \\
\hline & & & & & & & & & \multicolumn{2}{|c|}{ Rounded } & $\$$ & $21,450,000$ \\
\hline
\end{tabular}


Table E2.4A

10000kW - 25 kV Plan Cost Summary

\begin{tabular}{|c|c|c|c|c|c|c|c|c|c|c|c|c|}
\hline \multirow[b]{2}{*}{ No. Wind Turbines } & No. & \multirow[t]{2}{*}{ Units } & \multicolumn{2}{|c|}{$\begin{array}{l}\text { Avg. Cost } \\
\text { Per Unit }\end{array}$} & \multicolumn{2}{|r|}{$\begin{array}{c}\text { Labor \& } \\
\text { Material } \\
\text { Costs }\end{array}$} & \multicolumn{2}{|r|}{$\begin{array}{c}\text { Land \& } \\
\mathrm{R} / \mathrm{W}\end{array}$} & \multicolumn{2}{|c|}{$\begin{array}{l}\text { Clearing \& } \\
\text { Site Prep }\end{array}$} & & \multirow[t]{2}{*}{$\begin{array}{c}\text { Project } \\
\text { Totals }\end{array}$} \\
\hline & 5 & & & & & & & & & & & \\
\hline 10/12.5/14 MVA Transformers & 5 & ea. & $\$$ & 163,800 & $\$$ & 819,000 & & & & & & \\
\hline Underground Cables $1 / 0,4 / 0,500,750 \mathrm{Al}$ & 6,600 & feet & $\$$ & 23.33 & $\$$ & 154,000 & & & $\$$ & - & & \\
\hline Reclosers and Risers & 3 & ea & $\$$ & 25,733 & $\$$ & 77,200 & & & & & & \\
\hline Overhead 25 kV Line $-3 \times 795$ ACSR & 4,500 & feet & $\$$ & 21.84 & $\$$ & 98,300 & $\$$ & 13,500 & $\$$ & - & & \\
\hline Substation - 30/40/50 MVA PDS & 1 & ea. & & & $\$$ & $2,528,000$ & $\$$ & 15,000 & $\$$ & 2,400 & & \\
\hline Subtotal Electrical & & & & & $\$$ & $3,676,500$ & $\$$ & 28,500 & $\$$ & 2,400 & $\$$ & $3,707,400$ \\
\hline Generator Foundations & 5 & ea. & $\$$ & $1,600,000$ & $\$$ & $8,000,000$ & & & & & & \\
\hline Service Roads (Option 2) & 6,150 & feet & $\$$ & 111 & $\$$ & 680,000 & & & & & & \\
\hline Crane Pads & 5 & ea. & $\$$ & 317,000 & $\$$ & $1,585,000$ & & & & & & \\
\hline Subtotal Civil & & & & & $\$$ & $10,265,000$ & & & & & $\$$ & $10,265,000$ \\
\hline Communications RTUs & 10 & ea. & $\$$ & 8,050 & $\$$ & 80,500 & & & & & & \\
\hline Fiber Communications Lines & 11,100 & feet & $\$$ & 5.59 & $\$$ & 62,000 & & & & & & \\
\hline Communications Master Station & 1 & ea. & $\$$ & 66,700 & $\$$ & 66,700 & & & & & & \\
\hline Subtotal Communications & & & & & $\$$ & 209,200 & & & & & $\$$ & 209,200 \\
\hline Tower Lighting & 5 & ea. & $\$$ & 10,000 & $\$$ & 50,000 & & & & & $\$$ & 50,000 \\
\hline 184m Meteorological Towers & 2 & ea. & $\$$ & 80,000 & $\$$ & 160,000 & & & & & $\$$ & 160,000 \\
\hline 40' x 60' Maintenance Building & 1 & ea. & & & $\$$ & 156,000 & $\$$ & 15,000 & $\$$ & 2,400 & $\$$ & 173,400 \\
\hline Subtotal Infrastructure & & & & & $\$$ & 575,200 & $\$$ & 15,000 & $\$$ & 2,400 & $\$$ & 592,600 \\
\hline Subtotal Construction & & & & & $\$$ & $14,516,700$ & $\$$ & 43,500 & $\$$ & 4,800 & $\$$ & $14,565,000$ \\
\hline \multicolumn{13}{|l|}{ Project Overhead Costs } \\
\hline Licensing \& Permits & \multirow{2}{*}{\multicolumn{4}{|c|}{$50.0 \%$ of Land Costs }} & & & & & & & $\$$ & 5,000 \\
\hline Right-of-way Procurement & & & & & & & & & & & $\$$ & 23,400 \\
\hline Engineering & $7.0 \%$ & f Labor & Mate & rial & & & & & & & $\$$ & $1,016,200$ \\
\hline Surveying & $10,650 \mathrm{f}$ & & $\$$ & 2.00 & & & & & & & $\$$ & 21,300 \\
\hline Construction Inspection & & onths & $\$$ & 10,000 & & & & & & & $\$$ & 60,000 \\
\hline Owners Admin. Costs & & onths & $\$$ & 10,000 & & & & & & & $\$$ & 60,000 \\
\hline Subtotal Project Overhead Costs & & & & & & & & & & & $\$$ & $1,185,900$ \\
\hline Project Subtotal & & & & & & & & & & & $\$$ & $15,750,900$ \\
\hline Contingency & $20.0 \%$ & & & & & & & & & & $\$$ & $3,150,180$ \\
\hline Project Total & & & & & & & & & & & $\$$ & $18,901,080$ \\
\hline & & & & & & & & & Rou & Inded & $\$$ & $18,900,000$ \\
\hline
\end{tabular}


Table E2.4B

10000kW - 35 kV Plan Cost Summary

\begin{tabular}{|c|c|c|c|c|c|c|c|c|c|c|c|c|}
\hline \multirow[b]{2}{*}{ No. Wind Turbines } & No. & \multirow[t]{2}{*}{ Units } & \multicolumn{2}{|c|}{$\begin{array}{l}\text { Avg. Cost } \\
\text { Per Unit }\end{array}$} & \multicolumn{2}{|r|}{$\begin{array}{c}\text { Labor \& } \\
\text { Material } \\
\text { Costs }\end{array}$} & \multicolumn{2}{|r|}{$\begin{array}{c}\text { Land \& } \\
\mathrm{R} / \mathrm{W}\end{array}$} & \multicolumn{2}{|c|}{$\begin{array}{l}\text { Clearing \& } \\
\text { Site Prep }\end{array}$} & & \multirow[t]{2}{*}{$\begin{array}{c}\text { Project } \\
\text { Totals }\end{array}$} \\
\hline & 5 & & & & & & & & & & & \\
\hline 10/12.5/14 MVA Transformers & 5 & ea. & $\$$ & 174,000 & $\$$ & 870,000 & & & & & & \\
\hline Underground Cables $1 / 0,4 / 0,500,750 \mathrm{Al}$ & 6,600 & feet & $\$$ & 26.36 & $\$$ & 174,000 & & & $\$$ & - & & \\
\hline Reclosers and Risers & 3 & ea & $\$$ & 27,700 & $\$$ & 83,100 & & & & & & \\
\hline Overhead 35 kV Line $-3 \times 795$ ACSR & 4,500 & feet & $\$$ & 21.84 & $\$$ & 98,300 & $\$$ & 13,500 & $\$$ & - & & \\
\hline Substation - 30/40/50 MVA PDS & 1 & ea. & & & $\$$ & $2,729,000$ & $\$$ & 15,000 & $\$$ & 2,400 & & \\
\hline Subtotal Electrical & & & & & $\$$ & $3,954,400$ & $\$$ & 28,500 & $\$$ & 2,400 & $\$$ & $3,985,300$ \\
\hline Generator Foundations & 5 & ea. & $\$$ & $1,600,000$ & $\$$ & $8,000,000$ & & & & & & \\
\hline Service Roads (Option 2) & 6,150 & feet & $\$$ & 111 & $\$$ & 680,000 & & & & & & \\
\hline Crane Pads & 5 & ea. & $\$$ & 317,000 & $\$$ & $1,585,000$ & & & & & & \\
\hline Subtotal Civil & & & & & $\$$ & $10,265,000$ & & & & & $\$$ & $10,265,000$ \\
\hline Communications RTUs & 10 & ea. & $\$$ & 8,050 & $\$$ & 80,500 & & & & & & \\
\hline Fiber Communications Lines & 11,100 & feet & $\$$ & 5.59 & $\$$ & 62,000 & & & & & & \\
\hline Communications Master Station & 1 & ea. & $\$$ & 66,700 & $\$$ & 66,700 & & & & & & \\
\hline Subtotal Communications & & & & & $\$$ & 209,200 & & & & & $\$$ & 209,200 \\
\hline Tower Lighting & 5 & ea. & $\$$ & 10,000 & $\$$ & 50,000 & & & & & $\$$ & 50,000 \\
\hline 184m Meteorological Towers & 2 & ea. & $\$$ & 80,000 & $\$$ & 160,000 & & & & & $\$$ & 160,000 \\
\hline 40' x 60' Maintenance Building & 1 & ea. & & & $\$$ & 156,000 & $\$$ & 15,000 & $\$$ & 2,400 & $\$$ & 173,400 \\
\hline Subtotal Infrastructure & & & & & $\$$ & 575,200 & $\$$ & 15,000 & $\$$ & 2,400 & $\$$ & 592,600 \\
\hline Subtotal Construction & & & & & $\$$ & $14,794,600$ & $\$$ & 43,500 & $\$$ & 4,800 & $\$$ & $14,842,900$ \\
\hline \multicolumn{13}{|l|}{ Project Overhead Costs } \\
\hline Licensing \& Permits & \multirow{2}{*}{\multicolumn{4}{|c|}{$50.0 \%$ of Land Costs }} & & & & & & & $\$$ & 5,000 \\
\hline Right-of-way Procurement & & & & & & & & & & & $\$$ & 23,400 \\
\hline Engineering & $7.0 \%$ & f Labor & Mate & rial & & & & & & & $\$$ & $1,035,600$ \\
\hline Surveying & $10,650 \mathrm{f}$ & & $\$$ & 2.00 & & & & & & & $\$$ & 21,300 \\
\hline Construction Inspection & & onths & $\$$ & 10,000 & & & & & & & $\$$ & 60,000 \\
\hline Owners Admin. Costs & & onths & $\$$ & 10,000 & & & & & & & $\$$ & 60,000 \\
\hline Subtotal Project Overhead Costs & & & & & & & & & & & $\$$ & $1,205,300$ \\
\hline Project Subtotal & & & & & & & & & & & $\$$ & $16,048,200$ \\
\hline Contingency & $20.0 \%$ & & & & & & & & & & $\$$ & $3,209,640$ \\
\hline Project Total & & & & & & & & & & & $\$$ & $19,257,840$ \\
\hline & & & & & & & & & Rou & Inded & $\$$ & $19,260,000$ \\
\hline
\end{tabular}


Table E3.1A

750 kW - 15 kV Plan Padmount Transformer Cost Detail

\author{
Description \\ $11000 \mathrm{kVA}, 13.8 \mathrm{kV}-480 \mathrm{v}$, loop \\ $21000 \mathrm{kVA}, 13.8 \mathrm{kV}-480 \mathrm{v}$, end w/arresters
}

\begin{abstract}
Xfmr
Id
\end{abstract}

\begin{tabular}{|c|c|c|}
\hline 1 & 2 & $1000 \mathrm{kVA}, 13.8 \mathrm{kV}-480 \mathrm{v}$, end w/arresters \\
\hline 2 & 1 & 1000 kVA, 13.8 kV - 480v, loop \\
\hline 3 & 1 & 1000 kVA, 13.8 kV - 480v, loop \\
\hline 4 & 1 & 1000 kVA, 13.8 kV - 480v, loop \\
\hline 5 & 1 & 1000 kVA, 13.8 kV - 480v, loop \\
\hline 6 & 1 & 1000 kVA, 13.8 kV - 480v, loop \\
\hline 7 & 1 & 1000 kVA, 13.8 kV - 480v, loop \\
\hline 8 & 1 & 1000 kVA, 13.8 kV - 480v, loop \\
\hline 9 & 1 & 1000 kVA, 13.8 kV - 480v, loop \\
\hline 10 & 1 & 1000 kVA, 13.8 kV - 480v, loop \\
\hline 11 & 1 & 1000 kVA, 13.8 kV - 480v, loop \\
\hline 12 & 1 & 1000 kVA, 13.8 kV - 480v, loop \\
\hline 13 & 1 & 1000 kVA, 13.8 kV - 480v, loop \\
\hline 14 & 1 & 1000 kVA, 13.8 kV - 480v, loop \\
\hline 15 & 1 & 1000 kVA, 13.8 kV - 480v, loop \\
\hline 16 & 1 & 1000 kVA, 13.8 kV - 480v, loop \\
\hline 17 & 1 & 1000 kVA, 13.8 kV - 480v, loop \\
\hline 18 & 1 & 1000 kVA, 13.8 kV - 480v, loop \\
\hline 19 & 1 & 1000 kVA, 13.8 kV - 480v, loop \\
\hline 20 & 1 & 1000 kVA, 13.8 kV - 480v, loop \\
\hline 21 & 1 & 1000 kVA, 13.8 kV - 480v, loop \\
\hline 22 & 2 & $1000 \mathrm{kVA}, 13.8 \mathrm{kV}-480 \mathrm{v}$, end w/arresters \\
\hline 23 & 2 & $1000 \mathrm{kVA}, 13.8 \mathrm{kV}-480 \mathrm{v}$, end w/arresters \\
\hline 24 & 1 & 1000 kVA, 13.8 kV - 480v, loop \\
\hline 25 & 1 & 1000 kVA, 13.8 kV - 480v, loop \\
\hline 26 & 1 & 1000 kVA, 13.8 kV - 480v, loop \\
\hline 27 & 1 & 1000 kVA, 13.8 kV - 480v, loop \\
\hline 28 & 1 & 1000 kVA, 13.8 kV - 480v, loop \\
\hline 29 & 1 & 1000 kVA, 13.8 kV - 480v, loop \\
\hline 30 & 1 & 1000 kVA, 13.8 kV - 480v, loop \\
\hline 31 & 1 & 1000 kVA, 13.8 kV - 480v, loop \\
\hline 32 & 1 & 1000 kVA, 13.8 kV - 480v, loop \\
\hline 33 & 1 & 1000 kVA, 13.8 kV - 480v, loop \\
\hline 34 & 1 & 1000 kVA, 13.8 kV - 480v, loop \\
\hline 35 & 1 & 1000 kVA, 13.8 kV - 480v, loop \\
\hline 36 & 1 & 1000 kVA, 13.8 kV - 480v, loop \\
\hline 37 & 1 & 1000 kVA, 13.8 kV - 480v, loop \\
\hline 38 & 1 & 1000 kVA, 13.8 kV - 480v, loop \\
\hline 39 & 1 & 1000 kVA, 13.8 kV - 480v, loop \\
\hline 40 & 1 & 1000 kVA, 13.8 kV - 480v, loop \\
\hline 41 & 1 & 1000 kVA, 13.8 kV - 480v, loop \\
\hline
\end{tabular}

$\begin{array}{cc}\text { Rating } & \text { Cost } \\ 1000 & 17,300 \\ 1000 & 18,100 \\ \begin{array}{c}\text { Rating } \\ \text { kVA }\end{array} & \begin{array}{c}\text { Xfmr } \\ \text { Costs }\end{array}\end{array}$

$1000 \quad 18,100$

$1000 \quad 17,300$

$1000 \quad 17,300$

$1000 \quad 17,300$

$1000 \quad 17,300$

$1000 \quad 17,300$

$1000 \quad 17,300$

$1000 \quad 17,300$

$1000 \quad 17,300$

$1000 \quad 17,300$

$1000 \quad 17,300$

$1000 \quad 17,300$

$1000 \quad 17,300$

$1000 \quad 17,300$

$1000 \quad 17,300$

$1000 \quad 17,300$

$1000 \quad 17,300$

$1000 \quad 17,300$

$1000 \quad 17,300$

$1000 \quad 17,300$

$1000 \quad 17,300$

$1000 \quad 18,100$

$1000 \quad 18,100$

$1000 \quad 17,300$

$1000 \quad 17,300$

$1000 \quad 17,300$

$1000 \quad 17,300$

$1000 \quad 17,300$

$1000 \quad 17,300$

$1000 \quad 17,300$

$1000 \quad 17,300$

$1000 \quad 17,300$

$1000 \quad 17,300$

$1000 \quad 17,300$

$1000 \quad 17,300$

$1000 \quad 17,300$

$1000 \quad 17,300$

$1000 \quad 17,300$

$1000 \quad 17,300$

$1000 \quad 17,300$

$1000 \quad 17,300$ 
Table E3.1A

750 kW - 15 kV Plan Padmount Transformer Cost Detail

\section{Description \\ $11000 \mathrm{kVA}, 13.8 \mathrm{kV}-480 \mathrm{v}$, loop \\ $21000 \mathrm{kVA}, 13.8 \mathrm{kV}-480 \mathrm{v}$, end w/arresters}

\section{Xfmr \\ Id}

\begin{tabular}{ccl}
42 & 1 & 1000 kVA, 13.8 kV - 480v, loop \\
43 & 1 & $1000 \mathrm{kVA}, 13.8 \mathrm{kV}-480 \mathrm{v}$, loop \\
44 & 1 & $1000 \mathrm{kVA}, 13.8 \mathrm{kV}-480 \mathrm{v}$, loop \\
45 & 2 & $1000 \mathrm{kVA}, 13.8 \mathrm{kV}-480 \mathrm{v}$, end w/arresters \\
46 & 2 & $1000 \mathrm{kVA}, 13.8 \mathrm{kV}-480 \mathrm{v}$, end w/arresters \\
47 & 1 & $1000 \mathrm{kVA}, 13.8 \mathrm{kV}-480 \mathrm{v}$, loop \\
48 & 1 & $1000 \mathrm{kVA}, 13.8 \mathrm{kV}-480 \mathrm{v}$, loop \\
49 & 1 & $1000 \mathrm{kVA}, 13.8 \mathrm{kV}-480 \mathrm{v}$, loop \\
50 & 1 & $1000 \mathrm{kVA}, 13.8 \mathrm{kV}-480 \mathrm{v}$, loop \\
51 & 1 & $1000 \mathrm{kVA}, 13.8 \mathrm{kV}-480 \mathrm{v}$, loop \\
52 & 1 & $1000 \mathrm{kVA}, 13.8 \mathrm{kV}-480 \mathrm{v}$, loop \\
53 & 1 & $1000 \mathrm{kVA}, 13.8 \mathrm{kV}-480 \mathrm{v}$, loop \\
54 & 1 & $1000 \mathrm{kVA}, 13.8 \mathrm{kV}-480 \mathrm{v}$, loop \\
55 & 1 & $1000 \mathrm{kVA}, 13.8 \mathrm{kV}-480 \mathrm{v}$, loop \\
56 & 1 & $1000 \mathrm{kVA}, 13.8 \mathrm{kV}-480 \mathrm{v}$, loop \\
57 & 1 & $1000 \mathrm{kVA}, 13.8 \mathrm{kV}-480 \mathrm{v}$, loop \\
58 & 1 & $1000 \mathrm{kVA}, 13.8 \mathrm{kV}-480 \mathrm{v}$, loop \\
59 & 1 & $1000 \mathrm{kVA}, 13.8 \mathrm{kV}-480 \mathrm{v}$, loop \\
60 & 1 & $1000 \mathrm{kVA}, 13.8 \mathrm{kV}-480 \mathrm{v}$, loop \\
61 & 1 & $1000 \mathrm{kVA}, 13.8 \mathrm{kV}-480 \mathrm{v}$, loop \\
62 & 1 & $1000 \mathrm{kVA}, 13.8 \mathrm{kV}-480 \mathrm{v}$, loop \\
63 & 1 & $1000 \mathrm{kVA}, 13.8 \mathrm{kV}-480 \mathrm{v}$, loop \\
64 & 1 & $1000 \mathrm{kVA}, 13.8 \mathrm{kV}-480 \mathrm{v}$, loop \\
65 & 1 & $1000 \mathrm{kVA}, 13.8 \mathrm{kV}-480 \mathrm{v}$, loop \\
66 & 2 & $1000 \mathrm{kVA}, 13.8 \mathrm{kV}-480 \mathrm{v}$, end w/arresters \\
\hline Count & 66 &
\end{tabular}

\section{Rating Cost \\ $1000 \quad 17,300$ \\ $1000 \quad 18,100$}

\section{Rating Xfmr kVA Costs}

\section{Description}

\begin{tabular}{rr}
1000 & 17,300 \\
1000 & 17,300 \\
1000 & 17,300 \\
1000 & 18,100 \\
1000 & 18,100 \\
1000 & 17,300 \\
1000 & 17,300 \\
1000 & 17,300 \\
1000 & 17,300 \\
1000 & 17,300 \\
1000 & 17,300 \\
1000 & 17,300 \\
1000 & 17,300 \\
1000 & 17,300 \\
1000 & 17,300 \\
1000 & 17,300 \\
1000 & 17,300 \\
1000 & 17,300 \\
1000 & 17,300 \\
1000 & 17,300 \\
1000 & 17,300 \\
1000 & 17,300 \\
1000 & 17,300 \\
1000 & 17,300 \\
1000 & 18,100 \\
\hline Total & $1,146,600$ \\
Rounded & $\mathbf{1 , 1 4 7 , 0 0 0}$
\end{tabular}


Table E3.1B

750 kW - 25 kV Plan Padmount Transformer Cost Detail

\author{
Description \\ $11000 \mathrm{kVA}, 24.9 \mathrm{kV}-480 \mathrm{v}$, loop \\ $21000 \mathrm{kVA}, 24.9 \mathrm{kV}-480 \mathrm{v}$, end w/arresters
}

\begin{abstract}
Xfmr
Id
\end{abstract}

$\begin{array}{ccl}1 & 2 & \text { 1000 kVA, } 24.9 \mathrm{kV}-480 \mathrm{v} \text {, end w/arresters } \\ 2 & 1 & 1000 \mathrm{kVA}, 24.9 \mathrm{kV}-480 \mathrm{v} \text {, loop } \\ 3 & 1 & 1000 \mathrm{kVA}, 24.9 \mathrm{kV}-480 \mathrm{v} \text {, loop } \\ 4 & 1 & 1000 \mathrm{kVA}, 24.9 \mathrm{kV}-480 \mathrm{v} \text {, loop } \\ 5 & 1 & 1000 \mathrm{kVA}, 24.9 \mathrm{kV}-480 \mathrm{v} \text {, loop } \\ 6 & 1 & 1000 \mathrm{kVA}, 24.9 \mathrm{kV}-480 \mathrm{v} \text {, loop } \\ 7 & 1 & 1000 \mathrm{kVA}, 24.9 \mathrm{kV}-480 \mathrm{v} \text {, loop } \\ 8 & 1 & 1000 \mathrm{kVA}, 24.9 \mathrm{kV}-480 \mathrm{v} \text {, loop } \\ 9 & 1 & 1000 \mathrm{kVA}, 24.9 \mathrm{kV}-480 \mathrm{v} \text {, loop } \\ 10 & 1 & 1000 \mathrm{kVA}, 24.9 \mathrm{kV}-480 \mathrm{v} \text {, loop } \\ 11 & 1 & 1000 \mathrm{kVA}, 24.9 \mathrm{kV}-480 \mathrm{v} \text {, loop } \\ 12 & 1 & 1000 \mathrm{kVA}, 24.9 \mathrm{kV}-480 \mathrm{v} \text {, loop } \\ 13 & 1 & 1000 \mathrm{kVA}, 24.9 \mathrm{kV}-480 \mathrm{v} \text {, loop } \\ 14 & 1 & 1000 \mathrm{kVA}, 24.9 \mathrm{kV}-480 \mathrm{v} \text {, loop } \\ 15 & 1 & 1000 \mathrm{kVA}, 24.9 \mathrm{kV}-480 \mathrm{v} \text {, loop } \\ 16 & 1 & 1000 \mathrm{kVA}, 24.9 \mathrm{kV}-480 \mathrm{v} \text {, loop } \\ 17 & 1 & 1000 \mathrm{kVA}, 24.9 \mathrm{kV}-480 \mathrm{v} \text {, loop } \\ 18 & 1 & 1000 \mathrm{kVA}, 24.9 \mathrm{kV}-480 \mathrm{v} \text {, loop } \\ 19 & 1 & 1000 \mathrm{kVA}, 24.9 \mathrm{kV}-480 \mathrm{v} \text {, loop } \\ 20 & 1 & 1000 \mathrm{kVA}, 24.9 \mathrm{kV}-480 \mathrm{v} \text {, loop } \\ 21 & 1 & 1000 \mathrm{kVA}, 24.9 \mathrm{kV}-480 \mathrm{v} \text {, loop } \\ 22 & 2 & 1000 \mathrm{kVA}, 24.9 \mathrm{kV}-480 \mathrm{v} \text {, end w/arresters } \\ 23 & 2 & 1000 \mathrm{kVA}, 24.9 \mathrm{kV}-480 \mathrm{v} \text {, end w/arresters } \\ 24 & 1 & 1000 \mathrm{kVA}, 24.9 \mathrm{kV}-480 \mathrm{v} \text {, loop } \\ 25 & 1 & 1000 \mathrm{kVA}, 24.9 \mathrm{kV}-480 \mathrm{v} \text {, loop } \\ 26 & 1 & 1000 \mathrm{kVA}, 24.9 \mathrm{kV}-480 \mathrm{v} \text {, loop } \\ 27 & 1 & 1000 \mathrm{kVA}, 24.9 \mathrm{kV}-480 \mathrm{v} \text {, loop } \\ 28 & 1 & 1000 \mathrm{kVA}, 24.9 \mathrm{kV}-480 \mathrm{v} \text {, loop } \\ 29 & 1 & 1000 \mathrm{kVA}, 24.9 \mathrm{kV}-480 \mathrm{v} \text {, loop } \\ 30 & 1 & 1000 \mathrm{kVA}, 24.9 \mathrm{kV}-480 \mathrm{v} \text {, loop } \\ 31 & 1 & 1000 \mathrm{kVA}, 24.9 \mathrm{kV}-480 \mathrm{v} \text {, loop } \\ 32 & 1 & 1000 \mathrm{kVA}, 24.9 \mathrm{kV}-480 \mathrm{v} \text {, loop } \\ 33 & 1 & 1000 \mathrm{kVA}, 24.9 \mathrm{kV}-480 \mathrm{v} \text {, loop } \\ 34 & 1 & 1000 \mathrm{kVA}, 24.9 \mathrm{kV}-480 \mathrm{v} \text {, loop } \\ 35 & 1 & 1000 \mathrm{kVA}, 24.9 \mathrm{kV}-480 \mathrm{v} \text {, loop } \\ 36 & 1 & 1000 \mathrm{kVA}, 24.9 \mathrm{kV}-480 \mathrm{v} \text {, loop } \\ 37 & 1 & 1000 \mathrm{kVA}, 24.9 \mathrm{kV}-480 \mathrm{v} \text {, loop } \\ 38 & 1 & 1000 \mathrm{kVA}, 24.9 \mathrm{kV}-480 \mathrm{v} \text {, loop } \\ 39 & 1 & 1000 \mathrm{kVA}, 24.9 \mathrm{kV}-480 \mathrm{v} \text {, loop } \\ 41 & 1 & 1000 \mathrm{kVA}, 24.9 \mathrm{kV}-480 \mathrm{v} \text {, loop } \\ & 1 & 1000 \mathrm{kVA}, 24.9 \mathrm{kV}-480 \mathrm{v} \text {, loop } \\ & & \end{array}$

$\begin{array}{cc}\text { Rating } & \text { Cost } \\ 1000 & 17,400 \\ 1000 & 18,100 \\ \begin{array}{c}\text { Rating } \\ \text { kVA }\end{array} & \begin{array}{c}\text { Xfmr } \\ \text { Costs }\end{array}\end{array}$

$1000 \quad 18,100$

$1000 \quad 17,400$

$1000 \quad 17,400$

$1000 \quad 17,400$

$1000 \quad 17,400$

$1000 \quad 17,400$

$1000 \quad 17,400$

$1000 \quad 17,400$

$1000 \quad 17,400$

$1000 \quad 17,400$

$1000 \quad 17,400$

$1000 \quad 17,400$

$1000 \quad 17,400$

$1000 \quad 17,400$

$1000 \quad 17,400$

$1000 \quad 17,400$

$1000 \quad 17,400$

$1000 \quad 17,400$

$1000 \quad 17,400$

$1000 \quad 17,400$

$1000 \quad 17,400$

$1000 \quad 18,100$

$1000 \quad 18,100$

$1000 \quad 17,400$

$1000 \quad 17,400$

$1000 \quad 17,400$

$1000 \quad 17,400$

$1000 \quad 17,400$

$1000 \quad 17,400$

$1000 \quad 17,400$

$1000 \quad 17,400$

$1000 \quad 17,400$

$1000 \quad 17,400$

$1000 \quad 17,400$

$1000 \quad 17,400$

$1000 \quad 17,400$

$1000 \quad 17,400$

$1000 \quad 17,400$

$1000 \quad 17,400$

$1000 \quad 17,400$

$1000 \quad 17,400$ 
Table E3.1B

750 kW - 25 kV Plan Padmount Transformer Cost Detail

\section{Description \\ $11000 \mathrm{kVA}, 24.9 \mathrm{kV}-480 \mathrm{v}$, loop \\ $21000 \mathrm{kVA}, 24.9 \mathrm{kV}-480 \mathrm{v}$, end w/arresters}

\section{Xfmr}

Id

\begin{tabular}{ccl}
42 & 1 & 1000 kVA, 24.9 kV - 480v, loop \\
43 & 1 & $1000 \mathrm{kVA}, 24.9 \mathrm{kV}-480 \mathrm{v}$, loop \\
44 & 1 & $1000 \mathrm{kVA}, 24.9 \mathrm{kV}-480 \mathrm{v}$, loop \\
45 & 2 & $1000 \mathrm{kVA}, 24.9 \mathrm{kV}-480 \mathrm{v}$, end w/arresters \\
46 & 2 & $1000 \mathrm{kVA}, 24.9 \mathrm{kV}-480 \mathrm{v}$, end w/arresters \\
47 & 1 & $1000 \mathrm{kVA}, 24.9 \mathrm{kV}-480 \mathrm{v}$, loop \\
48 & 1 & $1000 \mathrm{kVA}, 24.9 \mathrm{kV}-480 \mathrm{v}$, loop \\
49 & 1 & $1000 \mathrm{kVA}, 24.9 \mathrm{kV}-480 \mathrm{v}$, loop \\
50 & 1 & $1000 \mathrm{kVA}, 24.9 \mathrm{kV}-480 \mathrm{v}$, loop \\
51 & 1 & $1000 \mathrm{kVA}, 24.9 \mathrm{kV}-480 \mathrm{v}$, loop \\
52 & 1 & $1000 \mathrm{kVA}, 24.9 \mathrm{kV}-480 \mathrm{v}$, loop \\
53 & 1 & $1000 \mathrm{kVA}, 24.9 \mathrm{kV}-480 \mathrm{v}$, loop \\
54 & 1 & $1000 \mathrm{kVA}, 24.9 \mathrm{kV}-480 \mathrm{v}$, loop \\
55 & 1 & $1000 \mathrm{kVA}, 24.9 \mathrm{kV}-480 \mathrm{v}$, loop \\
56 & 1 & $1000 \mathrm{kVA}, 24.9 \mathrm{kV}-480 \mathrm{v}$, loop \\
57 & 1 & $1000 \mathrm{kVA}, 24.9 \mathrm{kV}-480 \mathrm{v}$, loop \\
58 & 1 & $1000 \mathrm{kVA}, 24.9 \mathrm{kV}-480 \mathrm{v}$, loop \\
59 & 1 & $1000 \mathrm{kVA}, 24.9 \mathrm{kV}-480 \mathrm{v}$, loop \\
60 & 1 & $1000 \mathrm{kVA}, 24.9 \mathrm{kV}-480 \mathrm{v}$, loop \\
61 & 1 & $1000 \mathrm{kVA}, 24.9 \mathrm{kV}-480 \mathrm{v}$, loop \\
62 & 1 & $1000 \mathrm{kVA}, 24.9 \mathrm{kV}-480 \mathrm{v}$, loop \\
63 & 1 & $1000 \mathrm{kVA}, 24.9 \mathrm{kV}-480 \mathrm{v}$, loop \\
64 & 1 & $1000 \mathrm{kVA}, 24.9 \mathrm{kV}-480 \mathrm{v}$, loop \\
65 & 1 & $1000 \mathrm{kVA}, 24.9 \mathrm{kV}-480 \mathrm{v}$, loop \\
66 & 2 & $1000 \mathrm{kVA}, 24.9 \mathrm{kV}-480 \mathrm{v}$, end w/arresters \\
\hline Count & 66 &
\end{tabular}

\section{Rating Cost \\ $1000 \quad 17,400$ \\ $1000 \quad 18,100$}

\section{Rating Xfmr kVA Costs}

\section{Description}

\begin{tabular}{rr}
1000 & 17,400 \\
1000 & 17,400 \\
1000 & 17,400 \\
1000 & 18,100 \\
1000 & 18,100 \\
1000 & 17,400 \\
1000 & 17,400 \\
1000 & 17,400 \\
1000 & 17,400 \\
1000 & 17,400 \\
1000 & 17,400 \\
1000 & 17,400 \\
1000 & 17,400 \\
1000 & 17,400 \\
1000 & 17,400 \\
1000 & 17,400 \\
1000 & 17,400 \\
1000 & 17,400 \\
1000 & 17,400 \\
1000 & 17,400 \\
1000 & 17,400 \\
1000 & 17,400 \\
1000 & 17,400 \\
1000 & 17,400 \\
1000 & 18,100 \\
\hline Total & $1,152,600$ \\
Rounded & $\mathbf{1 , 1 5 3 , 0 0 0}$
\end{tabular}


Table E3.2A

2500 kW - 25 kV Plan Padmount Transformer Cost Detail

$\begin{array}{cccc}\text { Description } & \text { Rating } & \text { Cost } \\ 1 & 3000 \mathrm{kVA}, 24.9 \mathrm{kV}-690 \mathrm{v} \text {, loop } & 3000 & 33,600 \\ & 3000 \mathrm{kVA}, 24.9 \mathrm{kV}-690 \mathrm{v} \text {, end w/arresters } & 3000 & 34,800 \\ & & \\ & \text { Description } & \text { Rating } & \text { Xfmr } \\ & \text { kVA } & \text { Costs }\end{array}$

\begin{tabular}{cclll}
1 & 2 & $3000 \mathrm{kVA}, 24.9 \mathrm{kV}-690 \mathrm{v}$, end w/arresters & 3000 & 34,800 \\
2 & 1 & $3000 \mathrm{kVA}, 24.9 \mathrm{kV}-690 \mathrm{v}$, loop & 3000 & 33,600 \\
3 & 1 & $3000 \mathrm{kVA}, 24.9 \mathrm{kV}-690 \mathrm{v}$, loop & 3000 & 33,600 \\
4 & 1 & $3000 \mathrm{kVA}, 24.9 \mathrm{kV}-690 \mathrm{v}$, loop & 3000 & 33,600 \\
5 & 1 & $3000 \mathrm{kVA}, 24.9 \mathrm{kV}-690 \mathrm{v}$, loop & 3000 & 33,600 \\
6 & 1 & $3000 \mathrm{kVA}, 24.9 \mathrm{kV}-690 \mathrm{v}$, loop & 3000 & 33,600 \\
7 & 1 & $3000 \mathrm{kVA}, 24.9 \mathrm{kV}-690 \mathrm{v}$, loop & 3000 & 33,600 \\
8 & 1 & $3000 \mathrm{kVA}, 24.9 \mathrm{kV}-690 \mathrm{v}$, loop & 3000 & 33,600 \\
9 & 1 & $3000 \mathrm{kVA}, 24.9 \mathrm{kV}-690 \mathrm{v}$, loop & 3000 & 33,600 \\
10 & 1 & $3000 \mathrm{kVA}, 24.9 \mathrm{kV}-690 \mathrm{v}$, loop & 3000 & 33,600 \\
11 & 2 & $3000 \mathrm{kVA}, 24.9 \mathrm{kV}-690 \mathrm{v}$, end w/arresters & 3000 & 34,800 \\
12 & 1 & $3000 \mathrm{kVA}, 24.9 \mathrm{kV}-690 \mathrm{v}$, loop & 3000 & 33,600 \\
13 & 1 & $3000 \mathrm{kVA}, 24.9 \mathrm{kV}-690 \mathrm{v}$, loop & 3000 & 33,600 \\
14 & 1 & $3000 \mathrm{kVA}, 24.9 \mathrm{kV}-690 \mathrm{v}$, loop & 3000 & 33,600 \\
15 & 1 & $3000 \mathrm{kVA}, 24.9 \mathrm{kV}-690 \mathrm{v}$, loop & 3000 & 33,600 \\
16 & 1 & $3000 \mathrm{kVA}, 24.9 \mathrm{kV}-690 \mathrm{v}$, loop & 3000 & 33,600 \\
17 & 1 & $3000 \mathrm{kVA}, 24.9 \mathrm{kV}-690 \mathrm{v}$, loop & 3000 & 33,600 \\
18 & 1 & $3000 \mathrm{kVA}, 24.9 \mathrm{kV}-690 \mathrm{v}$, loop & 3000 & 33,600 \\
19 & 1 & $3000 \mathrm{kVA}, 24.9 \mathrm{kV}-690 \mathrm{v}$, loop & 3000 & 33,600 \\
20 & 2 & $3000 \mathrm{kVA}, 24.9 \mathrm{kV}-690 \mathrm{v}$, end w/arresters & 3000 & 34,800 \\
\hline Count & 20 & & Total & 675,600 \\
& & & Rounded & 676,000
\end{tabular}


Table E3.2B

2500 kW - 35 kV Plan Padmount Transformer Cost Detail

$\begin{array}{cccc}\text { Description } & \text { Rating } & \text { Cost } \\ 1 & 3000 \mathrm{kVA}, 34.5 \mathrm{kV}-690 \mathrm{v} \text {, loop } & 3000 & 35,300 \\ 2 & 3000 \mathrm{kVA}, 34.5 \mathrm{kV}-690 \mathrm{v} \text {, end w/arresters } & 3000 & 36,500 \\ & & \text { Rating } & \text { Xfmr } \\ & \text { Description } & \text { kVA } & \text { Costs }\end{array}$

\begin{tabular}{ccllr}
1 & 2 & $3000 \mathrm{kVA}, 34.5 \mathrm{kV}-690 \mathrm{v}$, end w/arresters & 3000 & 36,500 \\
2 & 1 & $3000 \mathrm{kVA}, 34.5 \mathrm{kV}-690 \mathrm{v}$, loop & 3000 & 35,300 \\
3 & 1 & $3000 \mathrm{kVA}, 34.5 \mathrm{kV}-690 \mathrm{v}$, loop & 3000 & 35,300 \\
4 & 1 & $3000 \mathrm{kVA}, 34.5 \mathrm{kV}-690 \mathrm{v}$, loop & 3000 & 35,300 \\
5 & 1 & $3000 \mathrm{kVA}, 34.5 \mathrm{kV}-690 \mathrm{v}$, loop & 3000 & 35,300 \\
6 & 1 & $3000 \mathrm{kVA}, 34.5 \mathrm{kV}-690 \mathrm{v}$, loop & 3000 & 35,300 \\
7 & 1 & $3000 \mathrm{kVA}, 34.5 \mathrm{kV}-690 \mathrm{v}$, loop & 3000 & 35,300 \\
8 & 1 & $3000 \mathrm{kVA}, 34.5 \mathrm{kV}-690 \mathrm{v}$, loop & 3000 & 35,300 \\
9 & 1 & $3000 \mathrm{kVA}, 34.5 \mathrm{kV}-690 \mathrm{v}$, loop & 3000 & 35,300 \\
10 & 1 & $3000 \mathrm{kVA}, 34.5 \mathrm{kV}-690 \mathrm{v}$, loop & 3000 & 35,300 \\
11 & 2 & $3000 \mathrm{kVA}, 34.5 \mathrm{kV}-690 \mathrm{v}$, end w/arresters & 3000 & 36,500 \\
12 & 1 & $3000 \mathrm{kVA}, 34.5 \mathrm{kV}-690 \mathrm{v}$, loop & 3000 & 35,300 \\
13 & 1 & $3000 \mathrm{kVA}, 34.5 \mathrm{kV}-690 \mathrm{v}$, loop & 3000 & 35,300 \\
14 & 1 & $3000 \mathrm{kVA}, 34.5 \mathrm{kV}-690 \mathrm{v}$, loop & 3000 & 35,300 \\
15 & 1 & $3000 \mathrm{kVA}, 34.5 \mathrm{kV}-690 \mathrm{v}$, loop & 3000 & 35,300 \\
16 & 1 & $3000 \mathrm{kVA}, 34.5 \mathrm{kV}-690 \mathrm{v}$, loop & 3000 & 35,300 \\
17 & 1 & $3000 \mathrm{kVA}, 34.5 \mathrm{kV}-690 \mathrm{v}$, loop & 3000 & 35,300 \\
18 & 1 & $3000 \mathrm{kVA}, 34.5 \mathrm{kV}-690 \mathrm{v}$, loop & 3000 & 35,300 \\
19 & 1 & $3000 \mathrm{kVA}, 34.5 \mathrm{kV}-690 \mathrm{v}$, loop & 3000 & 35,300 \\
20 & 2 & $3000 \mathrm{kVA}, 34.5 \mathrm{kV}-690 \mathrm{v}$, end w/arresters & 3000 & 36,500 \\
\hline Count & 20 & & Total & 709,600 \\
& & & Rounded & 710,000
\end{tabular}

E3 - 6 
Table E3.3A

5000 kW - 25 kV Plan Padmount Transformer Cost Detail

$\begin{array}{cccc} & \text { Description } & \text { Rating } & \text { Cost } \\ 1 & 7500 \mathrm{kVA}, 24.9 \mathrm{kV}-2400 \mathrm{v} \text {, loop } & 7500 & 117,300 \\ 2 & 7500 \mathrm{kVA}, 24.9 \mathrm{kV}-2400 \mathrm{v} \text {, end w/arresters } & 7500 & 118,600\end{array}$

\section{Xfmr}

Id

\begin{tabular}{ccl}
1 & 2 & 7500 kVA, 24.9 kV - 2400v, end w/arresters \\
2 & 1 & 7500 kVA, 24.9 kV - 2400v, loop \\
3 & 1 & $7500 \mathrm{kVA}, 24.9 \mathrm{kV}-2400 \mathrm{v}$, loop \\
4 & 1 & $7500 \mathrm{kVA}, 24.9 \mathrm{kV}-2400 \mathrm{v}$, loop \\
5 & 1 & $7500 \mathrm{kVA}, 24.9 \mathrm{kV}-2400 \mathrm{v}$, loop \\
6 & 1 & $7500 \mathrm{kVA}, 24.9 \mathrm{kV}-2400 \mathrm{v}$, loop \\
7 & 2 & $7500 \mathrm{kVA}, 24.9 \mathrm{kV}-2400 \mathrm{v}$, end w/arresters \\
8 & 1 & $7500 \mathrm{kVA}, 24.9 \mathrm{kV}-2400 \mathrm{v}$, loop \\
9 & 1 & $7500 \mathrm{kVA}, 24.9 \mathrm{kV}-2400 \mathrm{v}$, loop \\
10 & 2 & 7500 kVA, 24.9 kV - 2400v, end w/arresters \\
\cline { 1 - 1 } Count & 10 &
\end{tabular}

\section{Description}


Table E3.3B

5000 kW - 35 kV Plan Padmount Transformer Cost Detail

$\begin{array}{cccc} & \text { Description } & \text { Rating } & \text { Cost } \\ 1 & 7500 \mathrm{kVA}, 34.5 \mathrm{kV}-2400 \mathrm{v} \text {, loop } & 7500 & 118,900 \\ 2 & 7500 \mathrm{kVA}, 34.5 \mathrm{kV}-2400 \mathrm{v} \text {, end w/arresters } & 7500 & 121,100\end{array}$

\section{Xfmr}

Id

\begin{tabular}{ccl}
1 & 2 & $7500 \mathrm{kVA}, 34.5 \mathrm{kV}-2400 \mathrm{v}$, end w/arresters \\
2 & 1 & 7500 kVA, 34.5 kV - 2400v, loop \\
3 & 1 & $7500 \mathrm{kVA}, 34.5 \mathrm{kV}-2400 \mathrm{v}$, loop \\
4 & 1 & $7500 \mathrm{kVA}, 34.5 \mathrm{kV}-2400 \mathrm{v}$, loop \\
5 & 1 & $7500 \mathrm{kVA}, 34.5 \mathrm{kV}-2400 \mathrm{v}$, loop \\
6 & 1 & $7500 \mathrm{kVA}, 34.5 \mathrm{kV}-2400 \mathrm{v}$, loop \\
7 & 2 & $7500 \mathrm{kVA}, 34.5 \mathrm{kV}-2400 \mathrm{v}$, end w/arresters \\
8 & 1 & $7500 \mathrm{kVA}, 34.5 \mathrm{kV}-2400 \mathrm{v}$, loop \\
9 & 1 & $7500 \mathrm{kVA}, 34.5 \mathrm{kV}-2400 \mathrm{v}$, loop \\
10 & 2 & 7500 kVA, 34.5 kV - 2400v, end w/arresters \\
\cline { 1 - 1 } Count & 10 &
\end{tabular}

\section{Description}


Table E3.4A

10000 kW - 25 kV Plan Padmount Transformer Cost Detail

\begin{tabular}{llcc} 
& \multicolumn{1}{c}{ Description } & Rating & Cost \\
1 & 10/12.5 MVA, 24.9 kV -4160V, loop & $10 / 12.5 / 14$ & 161,700 \\
2 & $10 / 12.5 \mathrm{MVA}, 24.9 \mathrm{kV}-4160 \mathrm{v}$, end w/arresters & $10 / 12.5 / 14$ & 165,300
\end{tabular}

\section{Xfmr}

Id

\begin{tabular}{ccc}
1 & 2 & $10 / 12.5 \mathrm{MVA}, 24.9 \mathrm{kV}-4160 \mathrm{v}$, end w/arresters \\
2 & 1 & $10 / 12.5 \mathrm{MVA}, 24.9 \mathrm{kV}-4160 \mathrm{~V}$, loop \\
3 & 2 & $10 / 12.5 \mathrm{MVA}, 24.9 \mathrm{kV}-4160 \mathrm{v}$, end w/arresters \\
4 & 1 & $10 / 12.5 \mathrm{MVA}, 24.9 \mathrm{kV}-4160 \mathrm{~V}$, loop \\
5 & 2 & $10 / 12.5 \mathrm{MVA}, 24.9 \mathrm{kV}-4160 \mathrm{v}$, end w/arresters \\
\cline { 1 - 2 } Count & 5 &
\end{tabular}
Description

$\begin{array}{cc}\text { Rating } & \text { Xfmr } \\ \text { kVA } & \text { Costs }\end{array}$

$10 / 12.5 / 14 \quad 165,300$

$10 / 12.5 / 14 \quad 161,700$

$10 / 12.5 / 14 \quad 165,300$

$10 / 12.5 / 14 \quad 161,700$

$10 / 12.5 / 14 \quad 165,300$

Total 819,300

Rounded $\quad 819,000$

Table E3.4B

10000 kW - 35 kV Plan Padmount Transformer Cost Detail

$\begin{array}{cccc} & \text { Description } & \text { Rating } & \text { Cost } \\ 1 & \text { 10/12.5 MVA, 34.5 kV -4160V, loop } & 10 / 12.5 / 14 & 171,800 \\ 2 & 10 / 12.5 \mathrm{MVA}, 34.5 \mathrm{kV}-4160 \mathrm{v} \text {, end w/arresters } & 10 / 12.5 / 14 & 175,400 \\ & & \text { Rating } & \text { Xfmr } \\ & & \text { kVA } & \text { Costs }\end{array}$

Xfmr

Id

\begin{tabular}{cc}
1 & 2 \\
2 & 1 \\
3 & 2 \\
4 & 1 \\
5 & 2 \\
\hline Count & 5
\end{tabular}

10/12.5 MVA, 34.5 kV - 4160v, end w/arresters 10/12.5 MVA, 34.5 kV -4160V, loop 10/12.5 MVA, $34.5 \mathrm{kV}-4160 \mathrm{v}$, end w/arresters 10/12.5 MVA, 34.5 kV -4160V, loop 10/12.5 MVA, 34.5 kV - 4160v, end w/arresters

\begin{tabular}{ll}
$10 / 12.5 / 14$ & 175,400 \\
$10 / 12.5 / 14$ & 171,800 \\
$10 / 12.5 / 14$ & 175,400 \\
$10 / 12.5 / 14$ & 171,800 \\
$10 / 12.5 / 14$ & 175,400 \\
\hline Total & 869,800 \\
Rounded & $\mathbf{8 7 0 , 0 0 0}$
\end{tabular}


Table E4.1A

750 kW - 15 kV Plan Underground Cable Cost Detail

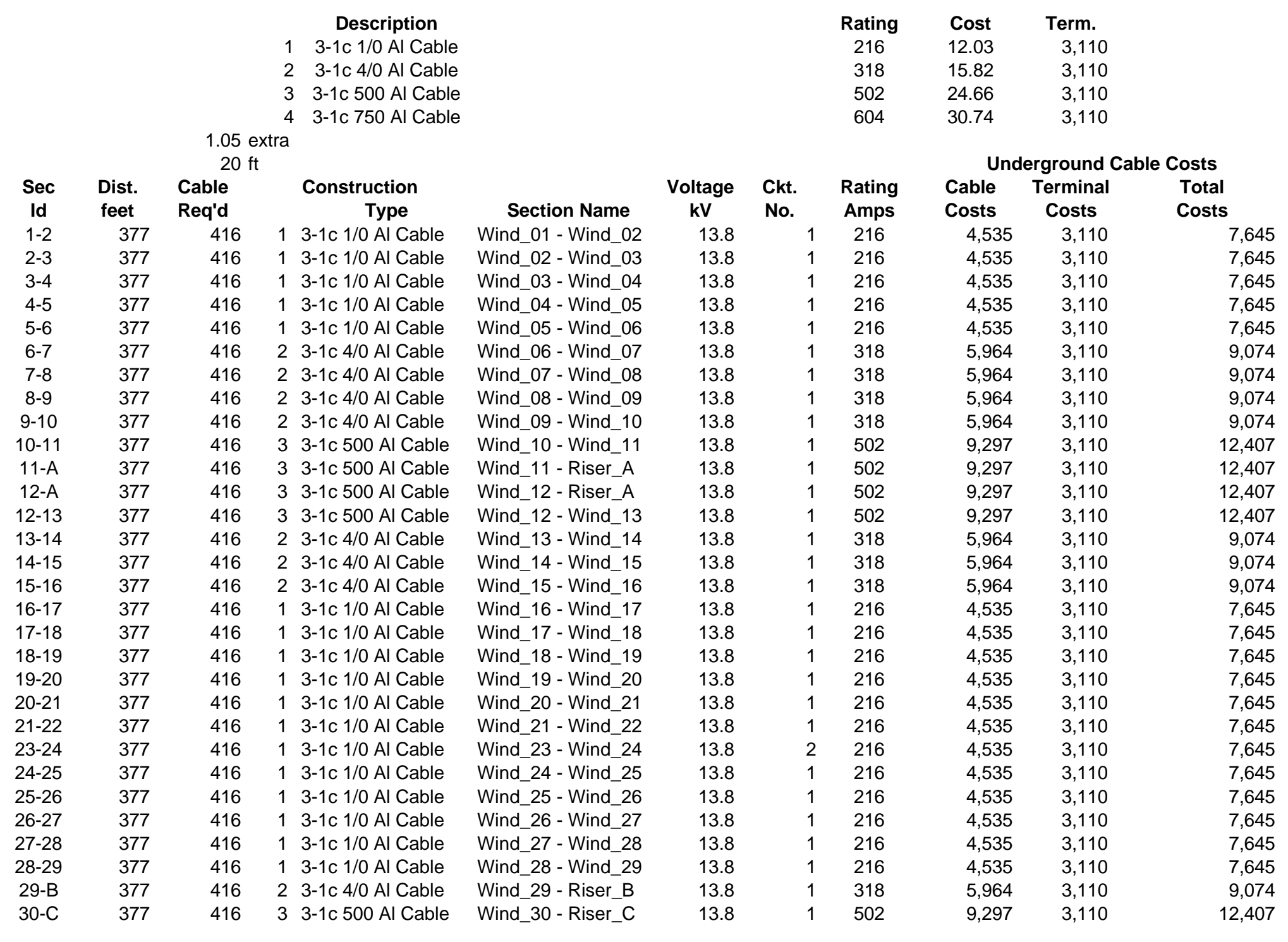


Table E4.1A

750 kW - 15 kV Plan Underground Cable Cost Detail

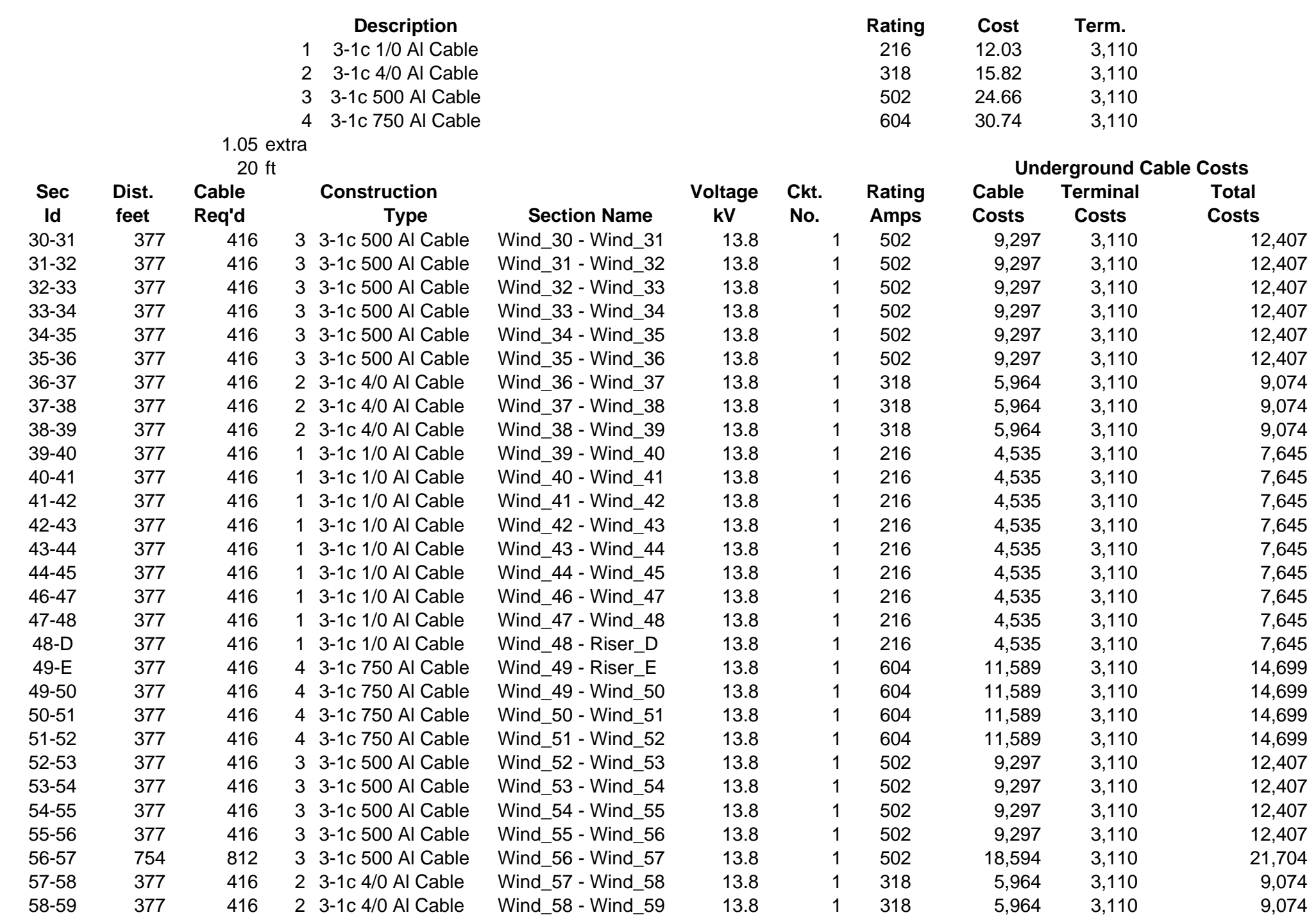


Table E4.1A

750 kW - 15 kV Plan Underground Cable Cost Detail

\begin{tabular}{|c|c|c|c|c|c|}
\hline & & & $\begin{array}{l}1 \\
2 \\
3 \\
4\end{array}$ & $\begin{array}{c}\text { Description } \\
\text { 3-1c 1/0 Al Cable } \\
\text { 3-1c 4/0 Al Cable } \\
\text { 3-1c } 500 \text { Al Cable } \\
\text { 3-1c } 750 \text { Al Cable }\end{array}$ & \\
\hline & & $\begin{array}{r}1.05 \mathrm{ex} \\
20 \mathrm{ft}\end{array}$ & & & \\
\hline $\begin{array}{l}\text { Sec } \\
\text { Id }\end{array}$ & $\begin{array}{l}\text { Dist. } \\
\text { feet }\end{array}$ & $\begin{array}{l}\text { Cable } \\
\text { Req'd }\end{array}$ & & $\begin{array}{c}\text { Construction } \\
\text { Type }\end{array}$ & Section Name \\
\hline $59-60$ & 377 & 416 & 2 & 3-1c 4/0 Al Cable & Wind_59 - Wind_60 \\
\hline $60-61$ & 377 & 416 & 1 & 3-1c 1/0 Al Cable & Wind_60 - Wind_61 \\
\hline $61-62$ & 377 & 416 & 1 & 3-1c 1/0 Al Cable & Wind_61 - Wind_62 \\
\hline $62-63$ & 377 & 416 & 1 & 3-1c 1/0 Al Cable & Wind_62 - Wind_63 \\
\hline $63-64$ & 754 & 812 & 1 & 3-1c 1/0 Al Cable & Wind_63 - Wind_64 \\
\hline $64-65$ & 377 & 416 & 1 & 3-1c 1/0 Al Cable & Wind_64 - Wind_65 \\
\hline $65-66$ & 377 & 416 & 1 & 3-1c 1/0 Al Cable & Wind_65 - Wind_66 \\
\hline Totals & 25,636 & 28,280 & & & \\
\hline $\begin{array}{l}\text { Rounded } \\
\text { Miles }\end{array}$ & 4.86 & 300 & & & \\
\hline
\end{tabular}

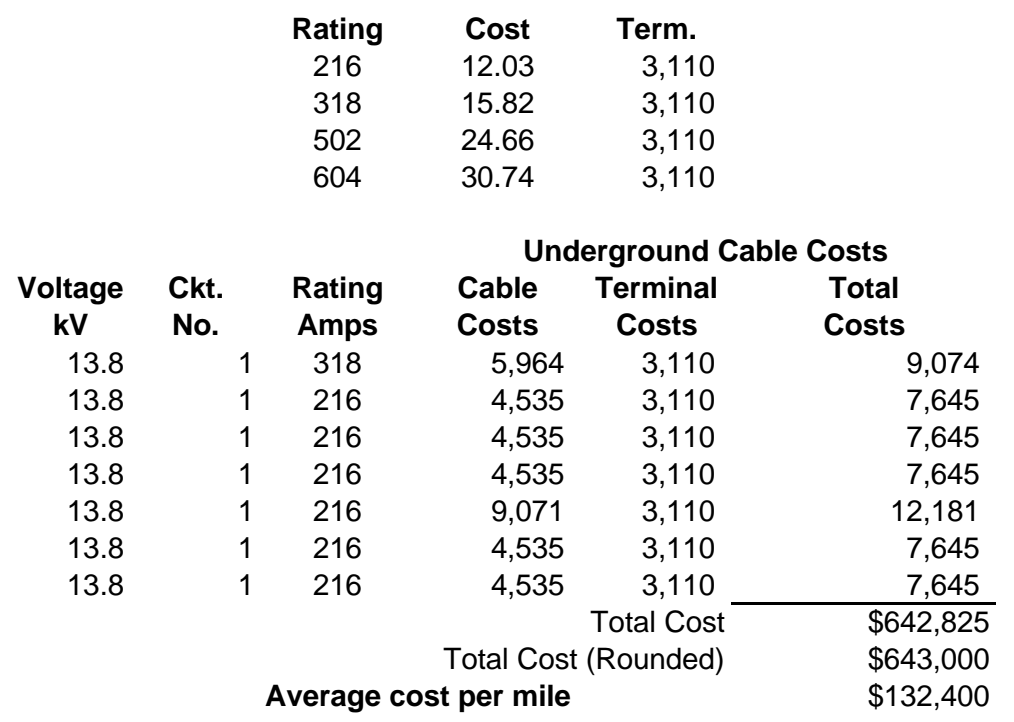


Table E4.1B

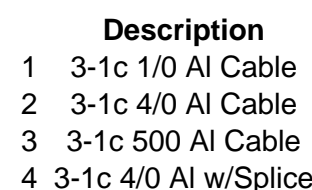

1.05 extra

$20 \mathrm{ft}$

\begin{tabular}{|c|c|}
\hline $\begin{array}{l}\text { Sec } \\
\text { Id }\end{array}$ & $\begin{array}{l}\text { Dist. } \\
\text { feet }\end{array}$ \\
\hline $1-2$ & 377 \\
\hline $2-3$ & 377 \\
\hline $3-4$ & 377 \\
\hline 4-5 & 377 \\
\hline $5-6$ & 377 \\
\hline $6-7$ & 377 \\
\hline 7-8 & 377 \\
\hline $8-9$ & 377 \\
\hline $9-10$ & 377 \\
\hline $10-11$ & 377 \\
\hline $11-A$ & 377 \\
\hline $12-A$ & 377 \\
\hline $12-13$ & 377 \\
\hline $13-14$ & 377 \\
\hline $14-15$ & 377 \\
\hline $15-16$ & 377 \\
\hline $16-17$ & 377 \\
\hline $17-18$ & 377 \\
\hline $18-19$ & 377 \\
\hline $19-20$ & 377 \\
\hline $20-21$ & 377 \\
\hline $21-22$ & 377 \\
\hline $23-24$ & 377 \\
\hline $24-25$ & 377 \\
\hline $25-26$ & 377 \\
\hline $26-27$ & 377 \\
\hline $27-28$ & 377 \\
\hline $28-29$ & 377 \\
\hline $29-B$ & 377 \\
\hline $30-C$ & 377 \\
\hline $30-31$ & 377 \\
\hline
\end{tabular}

q'd

3-1c $1 / 0$ Al Cable

1 3-1c 1/0 Al Cable

$416 \quad 13-1 \mathrm{c} 1 / 0 \mathrm{Al}$ Cable

$416 \quad 13-1 \mathrm{c} 1 / 0 \mathrm{Al}$ Cable

$416 \quad 1$ 3-1c 1/0 Al Cable

$416 \quad 1$ 3-1c 1/0 Al Cable

$416 \quad 13-1 \mathrm{c} 1 / 0$ Al Cable

$416 \quad 13-1 \mathrm{c} 1 / 0 \mathrm{Al}$ Cable

$416 \quad 1$ 3-1c 1/0 Al Cable

$416 \quad 13-1 \mathrm{c} 1 / 0$ Al Cable

$416 \quad 13-1 \mathrm{c} 1 / 0 \mathrm{Al}$ Cable

$416 \quad 1$ 3-1c $1 / 0$ Al Cable

$416 \quad 13-1$ c $1 / 0$ Al Cable

$416 \quad 1$ 3-1c 1/0 Al Cable

$416 \quad 1$ 3-1c 1/0 Al Cable

$416 \quad 13-1 \mathrm{c} 1 / 0$ Al Cable

$416 \quad 1$ 3-1c 1/0 Al Cable

$416 \quad 1 \quad 3-1 \mathrm{c} 1 / 0 \mathrm{Al}$ Cable

$416 \quad 1$ 3-1c 1/0 Al Cable

$416 \quad 1 \quad 3-1 \mathrm{c} 1 / 0 \mathrm{Al}$ Cable

$416 \quad 1$ 3-1c $1 / 0$ Al Cable

$416 \quad 1$ 3-1c $1 / 0$ Al Cable

$416 \quad 1$ 3-1c 1/0 Al Cable

$416 \quad 1$ 3-1c 1/0 Al Cable

$416 \quad 1$ 3-1c $1 / 0$ Al Cable

$416 \quad 1$ 3-1c 1/0 Al Cable

$416 \quad 1$ 3-1c $1 / 0$ Al Cable

$416 \quad 13-1 \mathrm{c} 1 / 0 \mathrm{Al}$ Cable

4162 3-1c 4/0 Al Cable

4162 3-1c 4/0 Al Cable
$416 \quad 13-1 \mathrm{c} 1 / 0$ Al Cable
750kW - 25 kV Plan Underground Cable Cost Detail

e

$\begin{array}{ccc}\text { Rating } & \text { Cost } & \text { Term. } \\ 216 & 13.98 & 3,535 \\ 318 & 17.70 & 3,535 \\ 502 & 27.42 & 3,535 \\ 318 & 17.70 & 4,750\end{array}$

Section Name
Wind_01 - Wind_02

Wind_02 - Wind_03

Wind 03 - Wind 04

Wind_04 - Wind_05

Wind 05 - Wind 06

Wind 06 - Wind 07

Wind_07 - Wind_08

Wind 08 - Wind 09

Wind 09 - Wind 10

Wind_10 - Wind_11

Wind 11 - Riser A

Wind 12 - Riser A

Wind_12 - Wind_13

Wind 13 - Wind 14

Wind 14 - Wind 15

Wind_15 - Wind_16

Wind_16 - Wind_17

Wind 17 - Wind 18

Wind_18 - Wind_19

Wind_19 - Wind_20

Wind 20 - Wind 21

Wind 21 - Wind 22

Wind_23 - Wind_24

Wind 24 - Wind 25

Wind 25 - Wind 26

Wind_26 - Wind_27

Wind 27 - Wind 28

Wind 28 - Wind 29

Wind_29 - Riser_B

Wind 30 - Riser $C$

Wind_30 - Wind_31

Voltage
kV
24.9
24.9
24.9
24.9
24.9
24.9
24.9
24.9
24.9
24.9
24.9
24.9
24.9
24.9
24.9
24.9
24.9
24.9
24.9
24.9
24.9
24.9
24.9
24.9
24.9
24.9
24.9
24.9
24.9
24.9
24.9

Underground Cable Costs

Cable Terminal Total

Costs Costs Costs

$\begin{array}{rrr}5,270 & 3,535 & \text { Costs } \\ 5,270 & 3,535 & 8,805\end{array}$

$5,270 \quad 3,535 \quad 8,805$

$\begin{array}{lll}5,270 & 3,535 & 8,805\end{array}$

$5,270 \quad 3,535 \quad 8,805$

$5,270 \quad 3,535 \quad 8,805$

$\begin{array}{lll}5,270 & 3,535 & 8,805\end{array}$

$\begin{array}{lll}5,270 & 3,535 & 8,805\end{array}$

$5,270 \quad 3,535 \quad 8,805$

$\begin{array}{lll}5,270 & 3,535 & 8,805\end{array}$

$\begin{array}{lll}5,270 & 3,535 & 8,805\end{array}$

$\begin{array}{lll}5,270 & 3,535 & 8,805\end{array}$

$\begin{array}{lll}5,270 & 3,535 & 8,805\end{array}$

$5,270 \quad 3,535 \quad 8,805$

$5,270 \quad 3,535 \quad 8,805$

$\begin{array}{lll}5,270 & 3,535 & 8,805\end{array}$

$5,270 \quad 3,535 \quad 8,805$

$5,270 \quad 3,535 \quad 8,805$

$\begin{array}{lll}5,270 & 3,535 & 8,805\end{array}$

$5,270 \quad 3,535 \quad 8,805$

$5,270 \quad 3,535 \quad 8,805$

$5,270 \quad 3,535 \quad 8,805$

$5,270 \quad 3,535 \quad 8,805$

$5,270 \quad 3,535 \quad 8,805$

$5,270 \quad 3,535 \quad 8,805$

$5,270 \quad 3,535 \quad 8,805$

$5,270 \quad 3,535 \quad 8,805$

$5,270 \quad 3,535 \quad 8,805$

$\begin{array}{lll}5,270 & 3,535 & 8,805\end{array}$

$5,270 \quad 3,535 \quad 8,805$

$\begin{array}{lll}6,673 & 3,535 & 10,208\end{array}$

$\begin{array}{lll}6,673 & 3,535 & 10,208\end{array}$ 
Table E4.1B

750kW - 25 kV Plan Underground Cable Cost Detail

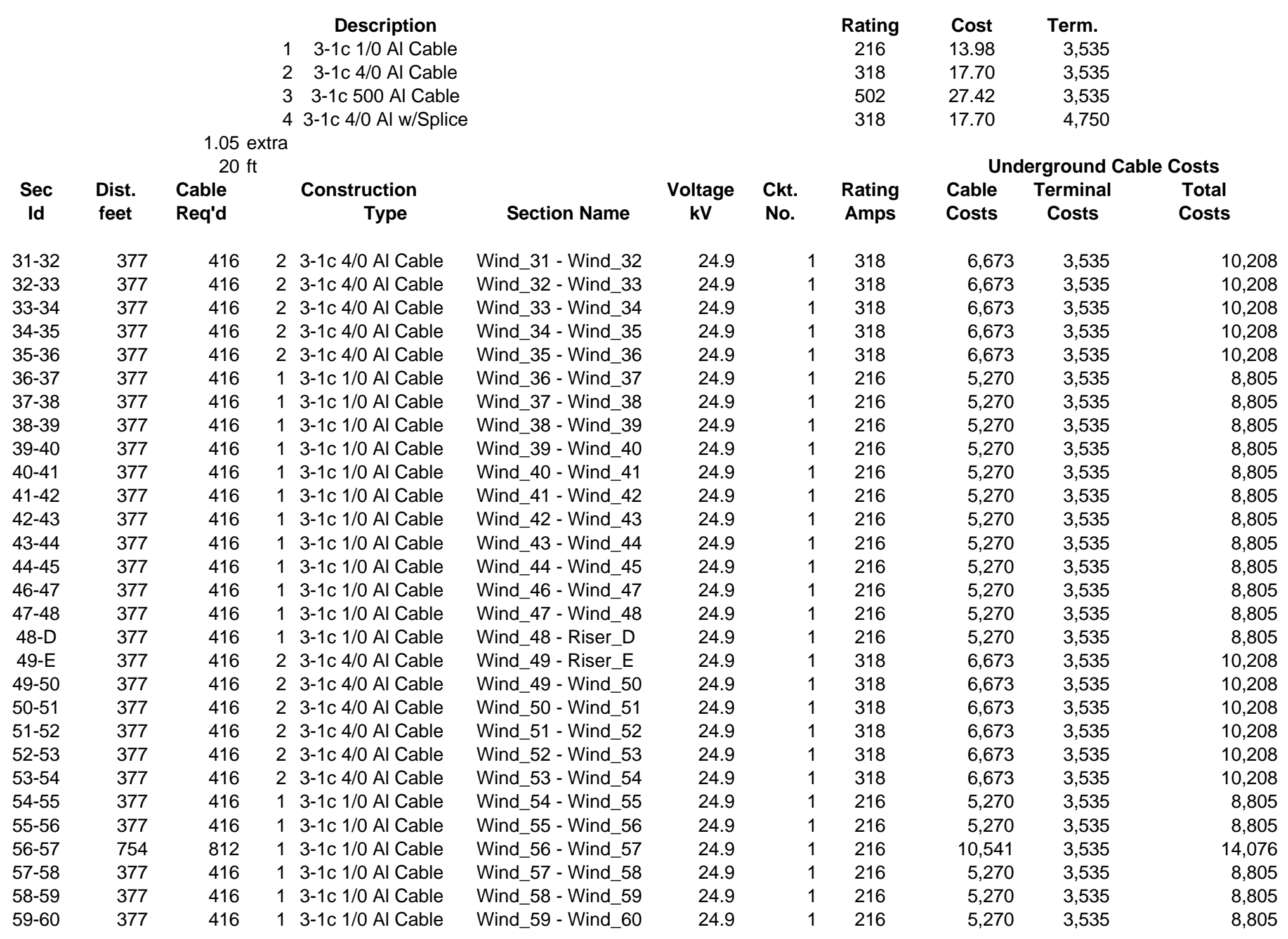


Table E4.1B

750kW - 25 kV Plan Underground Cable Cost Detail

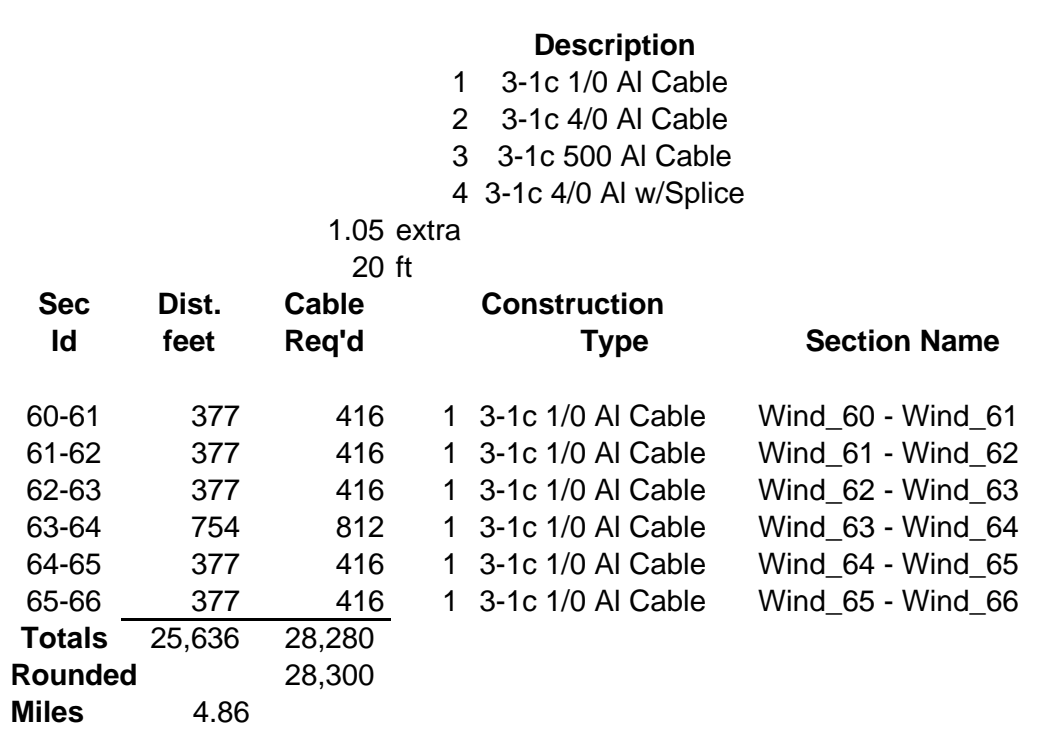

Voltage
kV

24.9
24.9
24.9
24.9
24.9
24.9

$\begin{array}{ccr}\text { Rating } & \text { Cost } & \text { Term. } \\ 216 & 13.98 & 3,535 \\ 318 & 17.70 & 3,535 \\ 502 & 27.42 & 3,535 \\ 318 & 17.70 & 4,750\end{array}$

\begin{tabular}{|c|c|c|c|c|c|}
\hline \multirow{2}{*}{\multicolumn{2}{|c|}{$\begin{array}{ll}\text { Itage } & \text { Ckt. } \\
\text { kV } & \text { No. }\end{array}$}} & \multirow[b]{2}{*}{$\begin{array}{c}\text { Rating } \\
\text { Amps }\end{array}$} & \\
\hline & & & $\begin{array}{l}\text { Cable } \\
\text { Costs }\end{array}$ & $\begin{array}{l}\text { Terminal } \\
\text { Costs }\end{array}$ & $\begin{array}{l}\text { Total } \\
\text { Costs }\end{array}$ \\
\hline 24.9 & 1 & 216 & 5,270 & 3,535 & 8,805 \\
\hline 24.9 & 1 & 216 & 5,270 & 3,535 & 8,805 \\
\hline 24.9 & 1 & 216 & 5,270 & 3,535 & 8,805 \\
\hline 4.9 & 1 & 216 & 10,541 & 3,535 & 14,076 \\
\hline 4.9 & 1 & 216 & 5,270 & 3,535 & 8,805 \\
\hline 4.9 & 1 & 216 & 5,270 & 3,535 & 8,805 \\
\hline & & & & & $\$ 609,933$ \\
\hline & & & Total Cost & (Rounded) & $\$ 610,000$ \\
\hline & & Average & t per mile & & $\$ 125,600$ \\
\hline
\end{tabular}


Table E4.2A

2500kW - 25 kV Plan Underground Cable Cost Detail

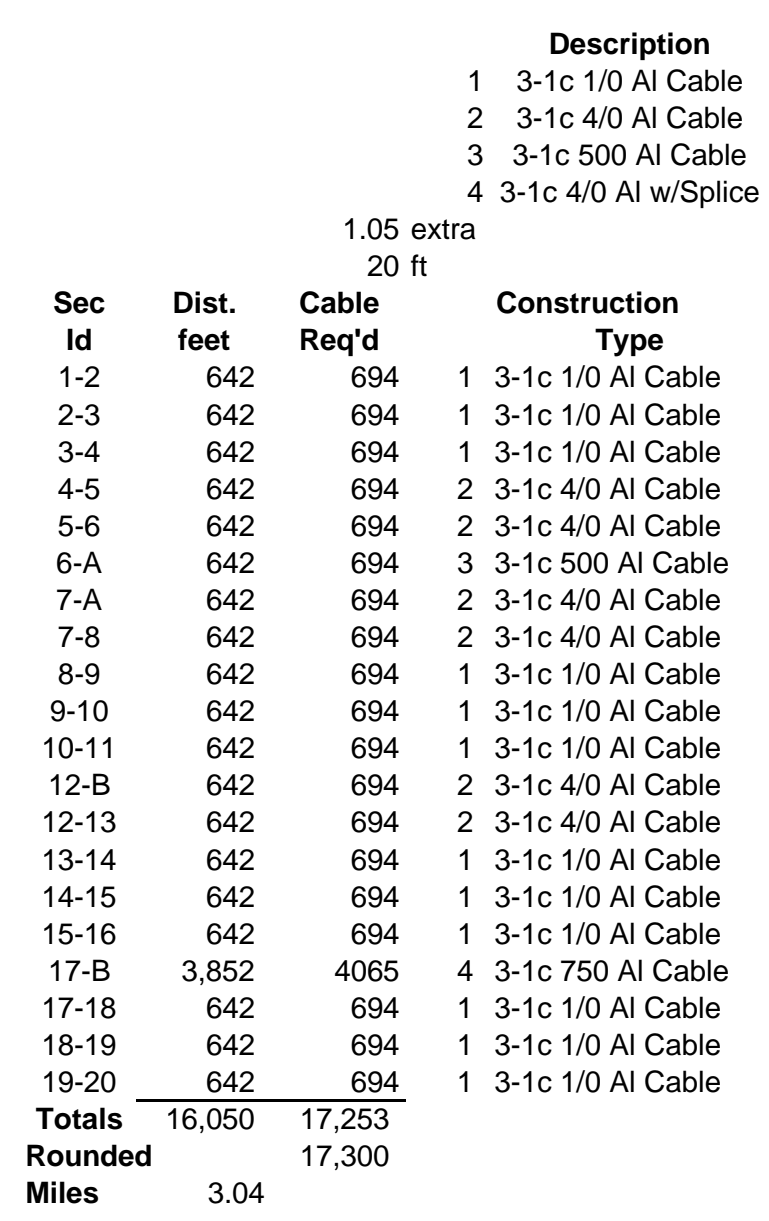

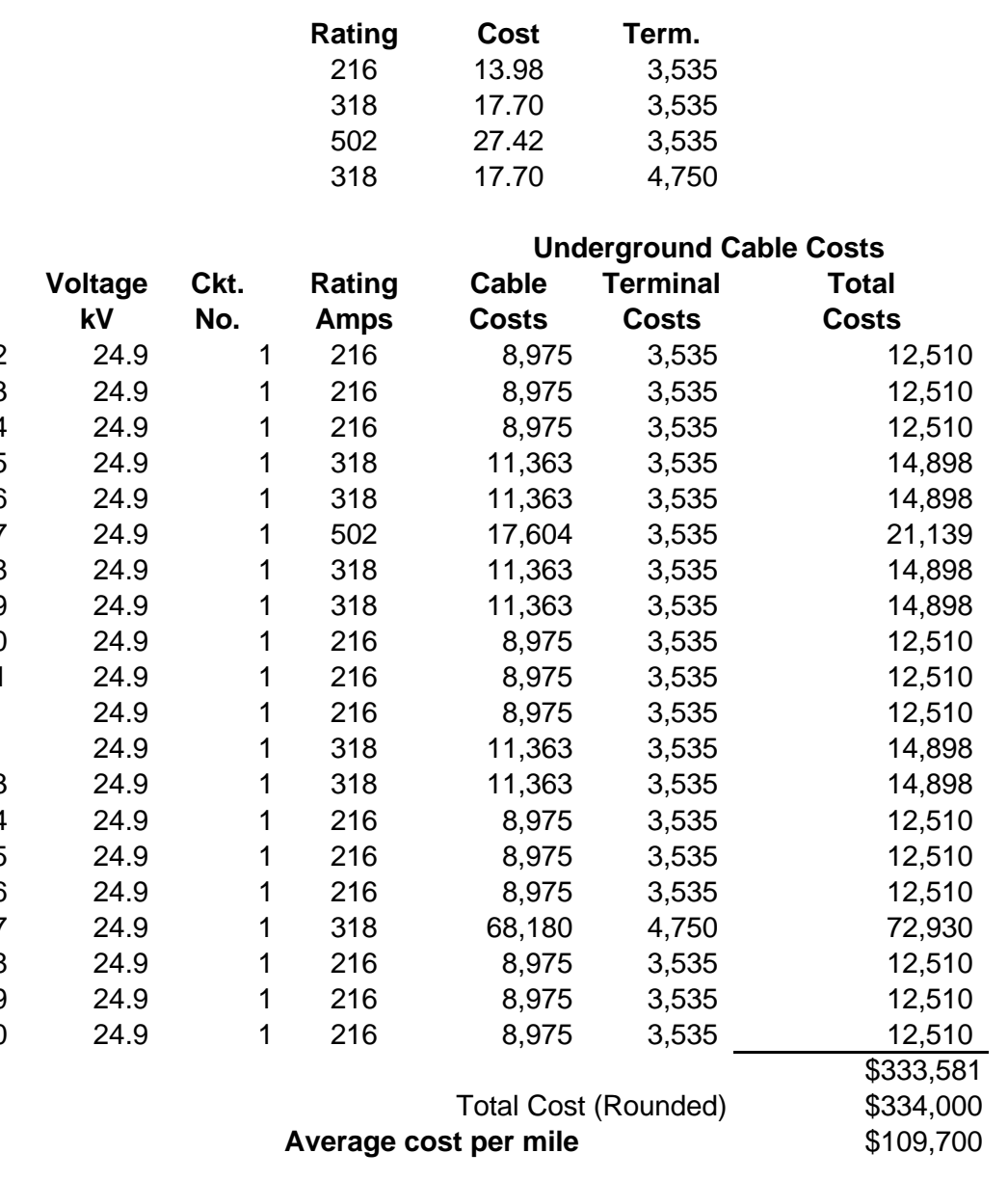


Table E4.2B

2500kW - 35 kV Plan Underground Cable Cost Detail

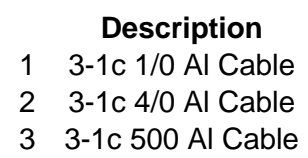

1.05 extra

\begin{tabular}{|c|c|c|c|c|}
\hline \multicolumn{5}{|c|}{1.05 extra } \\
\hline $\begin{array}{l}\text { Sec } \\
\text { Id }\end{array}$ & $\begin{array}{l}\text { Dist. } \\
\text { feet }\end{array}$ & $\begin{array}{l}\text { Cable } \\
\text { Req'd }\end{array}$ & & $\begin{array}{c}\text { Construction } \\
\text { Type }\end{array}$ \\
\hline $1-2$ & 642 & 694 & 1 & 3-1c 1/0 Al Cable \\
\hline $2-3$ & 642 & 694 & 1 & 3-1c 1/0 Al Cable \\
\hline $3-4$ & 642 & 694 & 1 & 3-1c 1/0 Al Cable \\
\hline $4-5$ & 642 & 694 & 1 & 3-1c 1/0 Al Cable \\
\hline $5-6$ & 642 & 694 & 1 & 3-1c 1/0 Al Cable \\
\hline $6-A$ & 642 & 694 & 2 & 3-1c 4/0 Al Cable \\
\hline 7-A & 642 & 694 & 1 & 3-1c 1/0 Al Cable \\
\hline $7-8$ & 642 & 694 & 1 & 3-1c 1/0 Al Cable \\
\hline $8-9$ & 642 & 694 & 1 & 3-1c 1/0 Al Cable \\
\hline $9-10$ & 642 & 694 & 1 & 3-1c 1/0 Al Cable \\
\hline $10-11$ & 642 & 694 & 1 & 3-1c 1/0 Al Cable \\
\hline $12-B$ & 642 & 694 & 3 & 3-1c 500 Al Cable \\
\hline $12-13$ & 642 & 694 & 3 & 3-1c 500 Al Cable \\
\hline $13-14$ & 642 & 694 & 2 & 3-1c 4/0 Al Cable \\
\hline $14-15$ & 642 & 694 & 2 & 3-1c 4/0 Al Cable \\
\hline $15-16$ & 642 & 694 & 1 & 3-1c 1/0 Al Cable \\
\hline $16-17$ & 642 & 694 & 1 & 3-1c 1/0 Al Cable \\
\hline $17-18$ & 642 & 694 & 1 & 3-1c 1/0 Al Cable \\
\hline $18-19$ & 642 & 694 & 1 & 3-1c 1/0 Al Cable \\
\hline $19-20$ & 642 & 694 & 1 & 3-1c 1/0 Al Cable \\
\hline Totals & 12,840 & 13,882 & & \\
\hline Rounded & & 13,900 & & \\
\hline Miles & 2.43 & & & \\
\hline
\end{tabular}

\begin{tabular}{|c|c|c|c|c|c|}
\hline & & $\begin{array}{c}\text { Rating } \\
216 \\
318 \\
502\end{array}$ & $\begin{array}{l}\text { Cost } \\
16.66 \\
22.91 \\
32.39\end{array}$ & $\begin{array}{r}\text { Term. } \\
5,479 \\
5,479 \\
5,479\end{array}$ & \\
\hline & & & \multicolumn{3}{|c|}{ Underground Cable Costs } \\
\hline $\begin{array}{c}\text { Voltage } \\
\text { kV }\end{array}$ & $\begin{array}{l}\text { Ckt. } \\
\text { No. }\end{array}$ & $\begin{array}{c}\text { Rating } \\
\text { Amps }\end{array}$ & $\begin{array}{l}\text { Cable } \\
\text { Costs }\end{array}$ & $\begin{array}{l}\text { Terminal } \\
\text { Costs }\end{array}$ & $\begin{array}{l}\text { Total } \\
\text { Costs }\end{array}$ \\
\hline 13.8 & 1 & 216 & 10,696 & 5,479 & 16,175 \\
\hline 13.8 & 1 & 216 & 10,696 & 5,479 & 16,175 \\
\hline 13.8 & 1 & 216 & 10,696 & 5,479 & 16,175 \\
\hline 13.8 & 1 & 216 & 10,696 & 5,479 & 16,175 \\
\hline 13.8 & 1 & 216 & 10,696 & 5,479 & 16,175 \\
\hline 13.8 & 1 & 318 & 14,708 & 5,479 & 20,187 \\
\hline 13.8 & 1 & 216 & 10,696 & 5,479 & 16,175 \\
\hline 13.8 & 1 & 216 & 10,696 & 5,479 & 16,175 \\
\hline 13.8 & 1 & 216 & 10,696 & 5,479 & 16,175 \\
\hline 13.8 & 1 & 216 & 10,696 & 5,479 & 16,175 \\
\hline 13.8 & 1 & 216 & 10,696 & 5,479 & 16,175 \\
\hline 13.8 & 1 & 502 & 20,794 & 5,479 & 26,273 \\
\hline 13.8 & 1 & 502 & 20,794 & 5,479 & 26,273 \\
\hline 13.8 & 1 & 318 & 14,708 & 5,479 & 20,187 \\
\hline 13.8 & 1 & 318 & 14,708 & 5,479 & 20,187 \\
\hline 13.8 & 1 & 216 & 10,696 & 5,479 & 16,175 \\
\hline 13.8 & 1 & 216 & 10,696 & 5,479 & 16,175 \\
\hline 13.8 & 1 & 216 & 10,696 & 5,479 & 16,175 \\
\hline 13.8 & 1 & 216 & 10,696 & 5,479 & 16,175 \\
\hline \multirow[t]{4}{*}{13.8} & 1 & 216 & 10,696 & 5,479 & 16,175 \\
\hline & & & & & $\$ 355,729$ \\
\hline & & & \multicolumn{2}{|c|}{ Total Cost (Rounded) } & $\$ 356,000$ \\
\hline & & Average & t per mile & & $\$ 146,300$ \\
\hline
\end{tabular}


Table E4.3A

5000kW - 25 kV Plan Underground Cable Cost Detail

\begin{abstract}
Description
1 3-1c 1/0 Al Cable

2 3-1c 4/0 Al Cable

3 3-1c 500 Al Cable
\end{abstract}

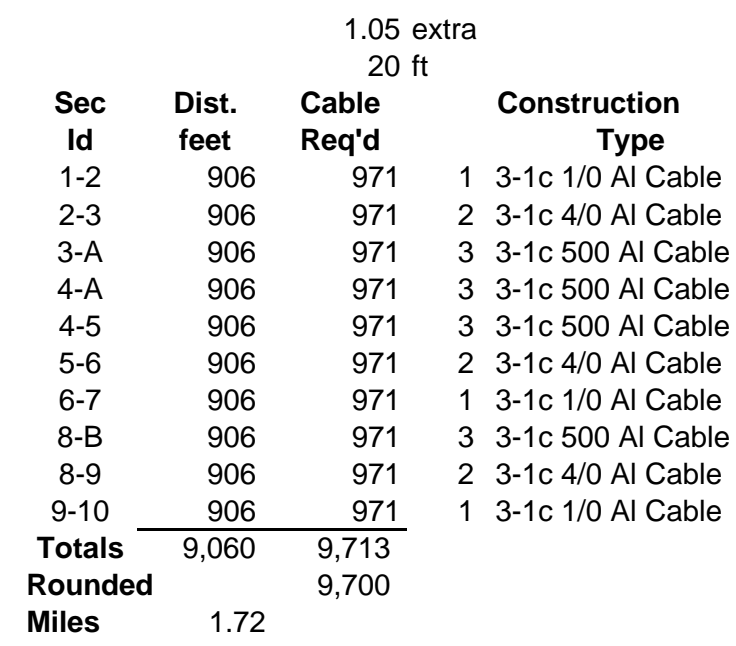

$\begin{array}{ccc}\text { Rating } & \text { Cost } & \text { Term. } \\ 216 & 13.98 & 3,535 \\ 318 & 17.70 & 3,535 \\ 502 & 27.42 & 3,535\end{array}$

Section Name
Wind_01 - Wind_02
Wind_02 - Wind_03
Wind_03 - Wind_04
Wind_04 - Wind_05
Wind_05 - Wind_06
Wind_06 - Wind_07
Wind_07 - Wind_08
Wind_08 - Wind_09
Wind_09 - Wind_10
Wind_10 - Wind_11

\begin{tabular}{cc} 
& \\
& \\
& \\
& \\
& \\
& \\
& Voltage \\
& kV \\
\hline 2 & 13.8 \\
3 & 13.8 \\
\hline 4 & 13.8 \\
\hline 5 & 13.8 \\
6 & 13.8 \\
\hline 7 & 13.8 \\
08 & 13.8 \\
9 & 13.8 \\
10 & 13.8 \\
1 & 13.8 \\
& \\
&
\end{tabular}

\begin{tabular}{ccccc} 
Ckt. & $\begin{array}{c}\text { Rating } \\
\text { No. }\end{array}$ & $\begin{array}{c}\text { Cable } \\
\text { Amps }\end{array}$ & $\begin{array}{c}\text { Costs } \\
\text { Terminal } \\
\text { Costs }\end{array}$ & $\begin{array}{c}\text { Total } \\
\text { Costs }\end{array}$ \\
1 & 216 & 12,666 & 3,535 & 16,201 \\
1 & 318 & 16,036 & 3,535 & 19,571 \\
1 & 502 & 24,843 & 3,535 & 28,378 \\
1 & 502 & 24,843 & 3,535 & 28,378 \\
1 & 502 & 24,843 & 3,535 & 28,378 \\
1 & 318 & 16,036 & 3,535 & 19,571 \\
1 & 216 & 12,666 & 3,535 & 16,201 \\
1 & 502 & 24,843 & 3,535 & 28,378 \\
1 & 318 & 16,036 & 3,535 & 19,571 \\
1 & 216 & 12,666 & 3,535 & 16,201 \\
\hline \multicolumn{5}{c}{ Total Cost (Rounded) } \\
\hline \multicolumn{5}{c}{ Average cost per mile }
\end{tabular}


Table E4.3B

5000kW - 35 kV Plan Underground Cable Cost Detail

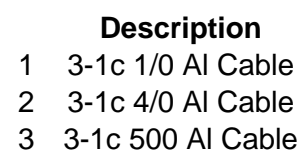

1.05 extra

\begin{tabular}{|c|c|c|c|c|}
\hline \multicolumn{5}{|c|}{1.05 extra } \\
\hline $\begin{array}{l}\text { Sec } \\
\text { Id }\end{array}$ & $\begin{array}{l}\text { Dist. } \\
\text { feet }\end{array}$ & $\begin{array}{l}\text { Cable } \\
\text { Req'd }\end{array}$ & & $\begin{array}{c}\text { Construction } \\
\text { Type }\end{array}$ \\
\hline $1-2$ & 906 & 971 & 1 & 3-1c 1/0 Al Cable \\
\hline $2-3$ & 906 & 971 & 1 & 3-1c 1/0 Al Cable \\
\hline $3-A$ & 906 & 971 & 2 & 3-1c 4/0 Al Cable \\
\hline $4-A$ & 906 & 971 & 3 & 3-1c 500 Al Cable \\
\hline $4-5$ & 906 & 971 & 2 & 3-1c 4/0 Al Cable \\
\hline $5-6$ & 906 & 971 & 1 & 3-1c 1/0 Al Cable \\
\hline $6-7$ & 906 & 971 & 1 & 3-1c 1/0 Al Cable \\
\hline $8-B$ & 906 & 971 & 2 & 3-1c 4/0 Al Cable \\
\hline $8-9$ & 906 & 971 & 1 & 3-1c 1/0 Al Cable \\
\hline $9-10$ & 906 & 971 & 1 & 3-1c 1/0 Al Cable \\
\hline Totals & 9,060 & $\overline{9,713}$ & & \\
\hline Rounded & & 9,700 & & \\
\hline Miles & 1.72 & & & \\
\hline
\end{tabular}

Section Name
Wind_01 - Wind_02
Wind_02 - Wind_03
Wind_03 - Wind_04
Wind_04 - Wind_05
Wind_05 - Wind_06
Wind_06 - Wind_07
Wind_07 - Wind_08
Wind_08 - Wind_09
Wind_09 - Wind_10
Wind_10 - Wind_11

\begin{tabular}{|c|c|c|c|c|c|}
\hline & & $\begin{array}{c}\text { Rating } \\
216 \\
318 \\
502\end{array}$ & $\begin{array}{l}\text { Cost } \\
16.66 \\
22.91 \\
32.39\end{array}$ & $\begin{array}{r}\text { Term. } \\
5,479 \\
5,479 \\
5,479\end{array}$ & \\
\hline & & & \multicolumn{3}{|c|}{ Underground Cable Costs } \\
\hline $\begin{array}{c}\text { Voltage } \\
\text { kV }\end{array}$ & $\begin{array}{l}\text { Ckt. } \\
\text { No. }\end{array}$ & $\begin{array}{l}\text { Rating } \\
\text { Amps }\end{array}$ & $\begin{array}{l}\text { Cable } \\
\text { Costs }\end{array}$ & $\begin{array}{l}\text { Terminal } \\
\text { Costs }\end{array}$ & $\begin{array}{l}\text { Total } \\
\text { Costs }\end{array}$ \\
\hline 13.8 & 1 & 216 & 15,094 & 5,479 & 20,573 \\
\hline 13.8 & 1 & 216 & 15,094 & 5,479 & 20,573 \\
\hline 13.8 & 1 & 318 & 20,756 & 5,479 & 26,235 \\
\hline 13.8 & 1 & 502 & 29,345 & 5,479 & 34,824 \\
\hline 13.8 & 1 & 318 & 20,756 & 5,479 & 26,235 \\
\hline 13.8 & 1 & 216 & 15,094 & 5,479 & 20,573 \\
\hline 13.8 & 1 & 216 & 15,094 & 5,479 & 20,573 \\
\hline 13.8 & 1 & 318 & 20,756 & 5,479 & 26,235 \\
\hline 13.8 & 1 & 216 & 15,094 & 5,479 & 20,573 \\
\hline \multirow[t]{4}{*}{13.8} & 1 & 216 & 15,094 & 5,479 & 20,573 \\
\hline & & & & & $\$ 236,968$ \\
\hline & & & \multicolumn{2}{|c|}{ Total Cost (Rounded) } & $\$ 237,000$ \\
\hline & \multicolumn{4}{|c|}{ Average cost per mile } & $\$ 138,100$ \\
\hline
\end{tabular}


Table E4.4A

10000kW - 25 kV Plan Underground Cable Cost Detail

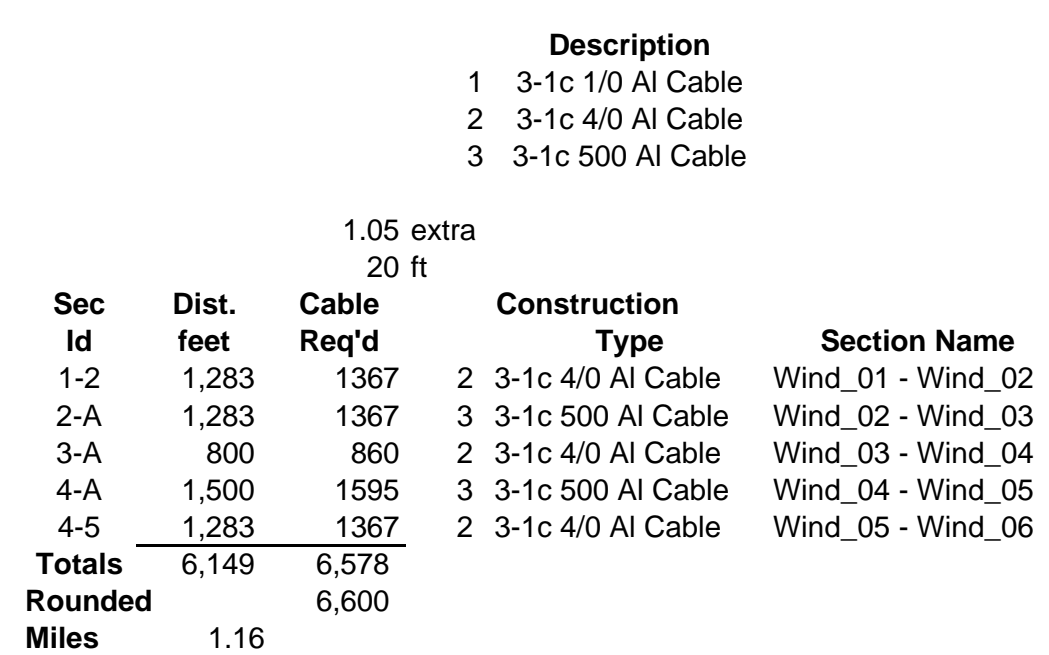

Voltage
kV
13.8
13.8
13.8
13.8
13.8

$\begin{array}{ccc}\text { Rating } & \text { Cost } & \text { Term. } \\ 216 & 13.98 & 3,535 \\ 318 & 17.70 & 3,535 \\ 502 & 27.42 & 3,535\end{array}$

\begin{tabular}{|c|c|c|c|c|}
\hline \multirow[b]{2}{*}{$\begin{array}{l}\text { Ckt. } \\
\text { No. }\end{array}$} & \multirow[b]{2}{*}{$\begin{array}{l}\text { Rating } \\
\text { Amps }\end{array}$} & \multicolumn{3}{|c|}{ Underground Cable Costs } \\
\hline & & $\begin{array}{l}\text { Cable } \\
\text { Costs }\end{array}$ & $\begin{array}{l}\text { Terminal } \\
\text { Costs }\end{array}$ & $\begin{array}{l}\text { Total } \\
\text { Costs }\end{array}$ \\
\hline .8 & 318 & 22,709 & 3,535 & 26,244 \\
\hline & 502 & 35,180 & 3,535 & 38,715 \\
\hline .8 & 318 & 14,160 & 3,535 & 17,695 \\
\hline & 502 & 41,130 & 3,535 & 44,665 \\
\hline & 318 & 22,709 & 3,535 & 26,244 \\
\hline & & & & $\$ 153,563$ \\
\hline & & Total Cos & ounded) & $\$ 154,000$ \\
\hline & Average & st per mile & & $\$ 131,900$ \\
\hline
\end{tabular}


Table E4.4B

10000kW - 35 kV Plan Underground Cable Cost Detail

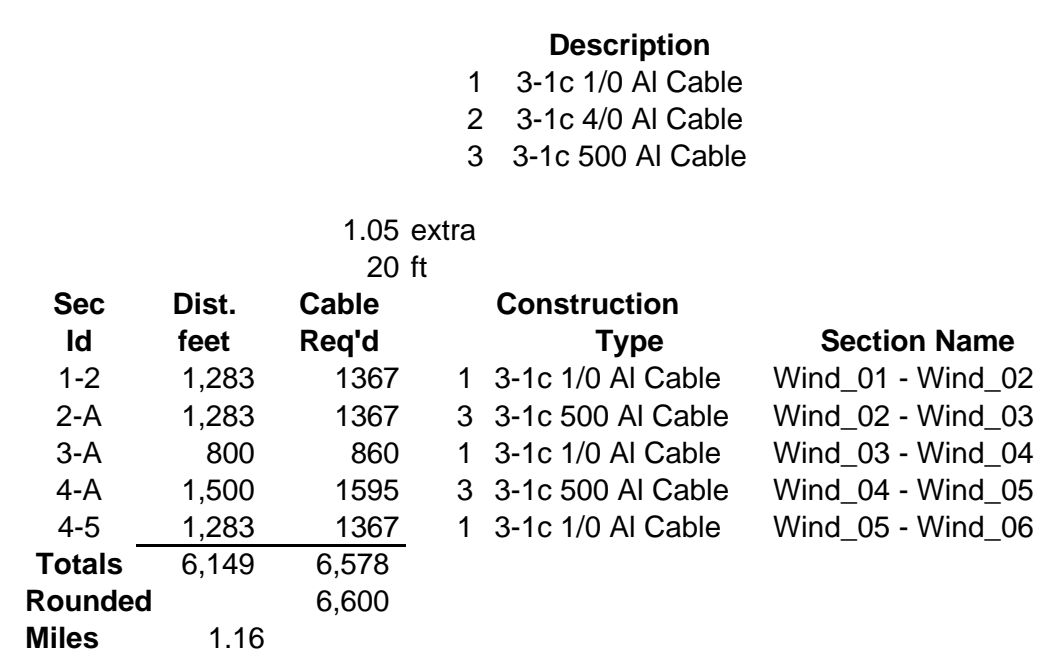

\begin{tabular}{|c|c|c|c|c|c|}
\hline & & $\begin{array}{c}\text { Rating } \\
216 \\
318 \\
502\end{array}$ & $\begin{array}{l}\text { Cost } \\
16.66 \\
22.91 \\
32.39\end{array}$ & $\begin{array}{r}\text { Term. } \\
5,479 \\
5,479 \\
5,479\end{array}$ & \\
\hline & & & \multicolumn{3}{|c|}{ Underground Cable Costs } \\
\hline $\begin{array}{c}\text { Voltage } \\
\text { kV }\end{array}$ & $\begin{array}{l}\text { Ckt. } \\
\text { No. }\end{array}$ & $\begin{array}{l}\text { Rating } \\
\text { Amps }\end{array}$ & $\begin{array}{l}\text { Cable } \\
\text { Costs }\end{array}$ & $\begin{array}{l}\text { Terminal } \\
\text { Costs }\end{array}$ & $\begin{array}{l}\text { Total } \\
\text { Costs }\end{array}$ \\
\hline 13.8 & 1 & 216 & 21,375 & 5,479 & 26,854 \\
\hline 13.8 & 1 & 502 & 41,556 & 5,479 & 47,035 \\
\hline 13.8 & 1 & 216 & 13,328 & 5,479 & 18,807 \\
\hline 13.8 & 1 & 502 & 48,585 & 5,479 & 54,064 \\
\hline \multirow[t]{4}{*}{13.8} & 1 & 216 & 21,375 & 5,479 & 26,854 \\
\hline & & & & & $\$ 173,614$ \\
\hline & & & \multicolumn{2}{|c|}{ Total Cost (Rounded) } & $\$ 174,000$ \\
\hline & & Average & t per mile & & $\$ 149,100$ \\
\hline
\end{tabular}


Table E5.1A

750 kW - 15 kV Plan Recloser and Riser Cost Detail

Description

VWE 15 kV Recloser with 1/0 Al Riser Cable VWE 15 kV Recloser with 4/0 Al Riser Cable VWE 15 kV Recloser with 500 Al Riser Cable VWE 15 kV Recloser with 750 Al Riser Cable VWE 15 kV Recl. W/(1) 750 and (1) 1/0 Riser

\begin{tabular}{cc} 
Riser & Type \\
& \\
Riser A1 & 3 \\
Riser A2 & 3 \\
Riser B & 2 \\
Riser C & 3 \\
Riser E & 5 \\
\hline Count & $\mathbf{5}$
\end{tabular}

VWE 15 kV Recloser with 500 Al Riser Cable VWE 15 kV Recloser with 500 Al Riser Cable VWE 15 kV Recloser with 4/0 Al Riser Cable VWE 15 kV Recloser with 500 Al Riser Cable VWE 15 kV Recl. W/(1) 750 and (1) 1/0 Riser
Description

$\begin{array}{cc}\text { Rating } & \text { Cost } \\ 560 & 24,500 \\ 560 & 24,600 \\ 560 & 24,800 \\ 560 & 25,000 \\ 560 & 26,500\end{array}$

Amps Costs

\begin{tabular}{cr}
560 & 24,800 \\
560 & 24,800 \\
560 & 24,600 \\
560 & 24,800 \\
560 & 26,500 \\
\hline Total & 125,500 \\
Rounded & $\mathbf{1 2 5 , 5 0 0}$
\end{tabular}

\section{Table E5.1B
750 kW - 25 kV Plan Recloser and Riser Cost Detail}

\section{Description}

VWE 25 kV Recloser with 1/0 Riser Cable VWE 25 kV Recloser with 4/0 Riser Cable VWE 25 kV Recl. W/(1) 4/0 and (1) 1/0 Riser

\begin{tabular}{ccc} 
Riser & Type & \multicolumn{1}{c}{ Description } \\
Riser A1 & 1 & VWE 25 kV Recloser with 1/0 Riser Cable \\
Riser A2 & 1 & VWE 25 kV Recloser with 1/0 Riser Cable \\
Riser B & 1 & VWE 25 kV Recloser with 1/0 Riser Cable \\
Riser C & 2 & VWE 25 kV Recloser with 4/0 Riser Cable \\
Riser E & 3 & VWE 25 kV Recl. W/(1) 4/0 and (1) 1/0 Riser \\
\cline { 1 - 1 } Count & $\mathbf{5}$ &
\end{tabular}

\begin{tabular}{cc} 
Rating & Cost \\
560 & 25,500 \\
560 & 25,600 \\
560 & 27,200 \\
& \\
Rating & Recloser \\
Amps & Costs \\
& \\
& \\
560 & 25,500 \\
560 & 25,500 \\
560 & 25,500 \\
560 & 25,600 \\
560 & 27,200 \\
\hline Total & 129,300 \\
Rounded & $\mathbf{1 2 9 , 3 0 0}$
\end{tabular}


Table E5.2A

2500 kW - 25 kV Plan Recloser and Riser Cost Detail

Description

1 VWE 25 kV Recloser with 4/0 Riser Cable

2 VWE 25 kV Recloser with 500 Riser Cable

$\begin{array}{cc}\text { Rating } & \text { Cost } \\ 560 & 25,600 \\ 560 & 25,800\end{array}$

Rating Recloser

Amps Costs

\begin{tabular}{lr}
560 & 25,800 \\
560 & 25,600 \\
560 & 25,600 \\
560 & 25,600 \\
\hline Total & 102,600 \\
Rounded & $\mathbf{1 0 2 , 6 0 0}$
\end{tabular}

Table E5.2B

2500 kW - 35 kV Plan Recloser and Riser Cost Detail

Description

1 VWE 35 kV Recloser with 1/0 Riser Cable

2 VWE 35 kV Recloser with 4/0 Riser Cable

3 VWE 35 kV Recloser with 500 Riser Cable

$\begin{array}{cc}\text { Rating } & \text { Cost } \\ 560 & 27,500 \\ 560 & 27,600 \\ 560 & 27,800\end{array}$

Rating Recloser

Amps Costs

\begin{tabular}{ll}
\multicolumn{1}{c}{560} & 27,600 \\
560 & 27,500 \\
560 & 27,800 \\
\hline Total & 82,900 \\
Rounded & $\mathbf{8 2 , 9 0 0}$
\end{tabular}


Table E5.3A

5000 kW - 25 kV Plan Recloser and Riser Cost Detail

$\begin{array}{cccc} & \text { Description } & \text { Rating } & \text { Cost } \\ 1 & \text { VWE 25 kV Recloser with 4/0 Riser Cable } & 560 & 25,600 \\ 2 & \text { VWE 25 kV Recloser with 500 Riser Cable } & 560 & 25,800 \\ & & & \\ & & \text { Rating } & \text { Recloser } \\ \text { Type } & \text { Description } & \text { Amps } & \text { Costs }\end{array}$

\begin{tabular}{ccccc} 
Riser & Type & \multicolumn{1}{c}{ Description } & $\begin{array}{c}\text { Rating } \\
\text { Amps }\end{array}$ & $\begin{array}{c}\text { Recloser } \\
\text { Costs }\end{array}$ \\
& & & & \\
Riser A1 & 2 & VWE 25 kV Recloser with 500 Riser Cable & 560 & 25,800 \\
Riser A2 & 2 & VWE 25 kV Recloser with 500 Riser Cable & 560 & 25,800 \\
Riser B & 2 & VWE 25 kV Recloser with 500 Riser Cable & 560 & 25,800 \\
\hline Count & $\mathbf{3}$ & & Total & 77,400 \\
& & & Rounded & $\mathbf{7 7 , 4 0 0}$
\end{tabular}

Table E5.3B

5000 kW - 35 kV Plan Recloser and Riser Cost Detail

$\begin{array}{cc} & \text { Description } \\ 1 & \text { VWE } 35 \text { kV Recloser with 1/0 Riser Cable } \\ 2 & \text { VWE 35 kV Recloser with 4/0 Riser Cable } \\ 3 & \text { VWE 35 kV Recloser with 500 Riser Cable }\end{array}$

Riser Type

Riser A1 2 VWE 35 kV Recloser with 4/0 Riser Cable

Riser A2 2 VWE 35 kV Recloser with 4/0 Riser Cable

$\begin{array}{cc}\text { Riser B } & 2 \\ \text { Count } & 3\end{array}$ VWE 35 kV Recloser with 4/0 Riser Cable

$\begin{array}{cc}\text { Rating } & \text { Cost } \\ 560 & 27,500 \\ 560 & 27,600 \\ 560 & 27,800\end{array}$

Rating Recloser

Amps Costs

\begin{tabular}{ll}
560 & 27,600 \\
560 & 27,600 \\
560 & 27,600 \\
\hline Total & 82,800 \\
Rounded & $\mathbf{8 2 , 8 0 0}$
\end{tabular}


Table E5.4A

10000 kW - 25 kV Plan Recloser and Riser Cost Detail

\section{Description \\ 1 VWE 25 kV Recloser with 4/0 Riser Cable \\ 2 VWE 25 kV Recloser with 500 Riser Cable}

$\begin{array}{cc}\text { Rating } & \text { Cost } \\ 560 & 25,600 \\ 560 & 25,800\end{array}$

Rating Recloser Amps Costs

\begin{tabular}{ll}
\multicolumn{1}{c}{560} & 25,800 \\
560 & 25,600 \\
560 & 25,800 \\
\hline Total & 77,200 \\
Rounded & $\mathbf{7 7 , 2 0 0}$
\end{tabular}

Table E5.4B

10000 kW - 35 kV Plan Recloser and Riser Cost Detail

$\begin{array}{cc} & \text { Description } \\ 1 & \text { VWE } 35 \text { kV Recloser with 1/0 Riser Cable } \\ 2 & \text { VWE } 35 \text { kV Recloser with 4/0 Riser Cable } \\ 3 & \text { VWE 35 kV Recloser with 500 Riser Cable }\end{array}$

Riser Type

Description

$\begin{array}{cc}\text { Rating } & \text { Cost } \\ 560 & 27,500 \\ 560 & 27,600 \\ 560 & 27,800 \\ & \\ \text { Rating } & \text { Recloser } \\ \text { Amps } & \text { Costs }\end{array}$

\begin{tabular}{ll}
560 & 27,800 \\
560 & 27,500 \\
560 & 27,800 \\
\hline Total & 83,100 \\
Rounded & $\mathbf{8 3 , 1 0 0}$
\end{tabular}


Table E6.1A

750 kW - 15 kV Plan Overhead Line Cost Detail

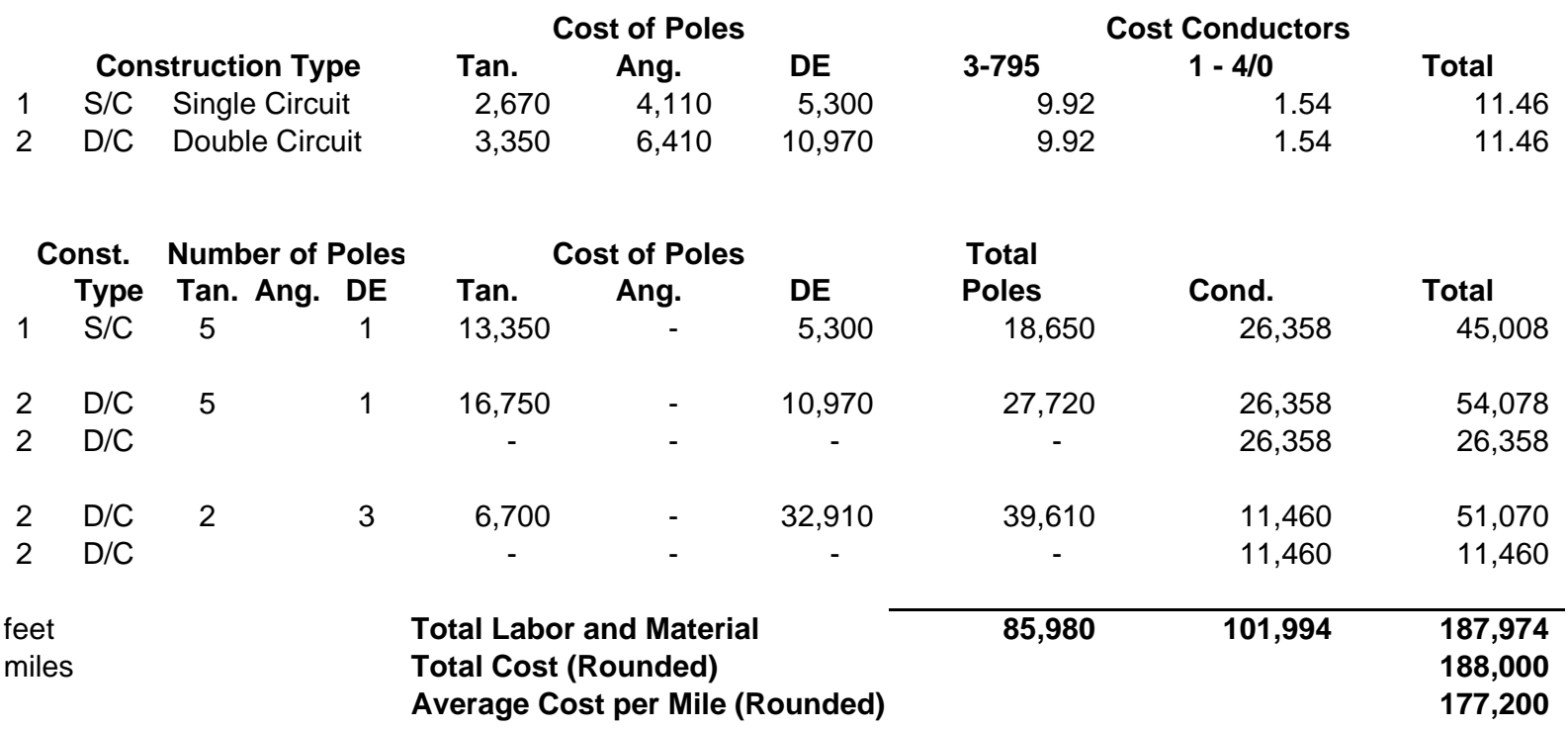


Table E6.1B

750 kW - 25 kV Plan Overhead Line Cost Detail

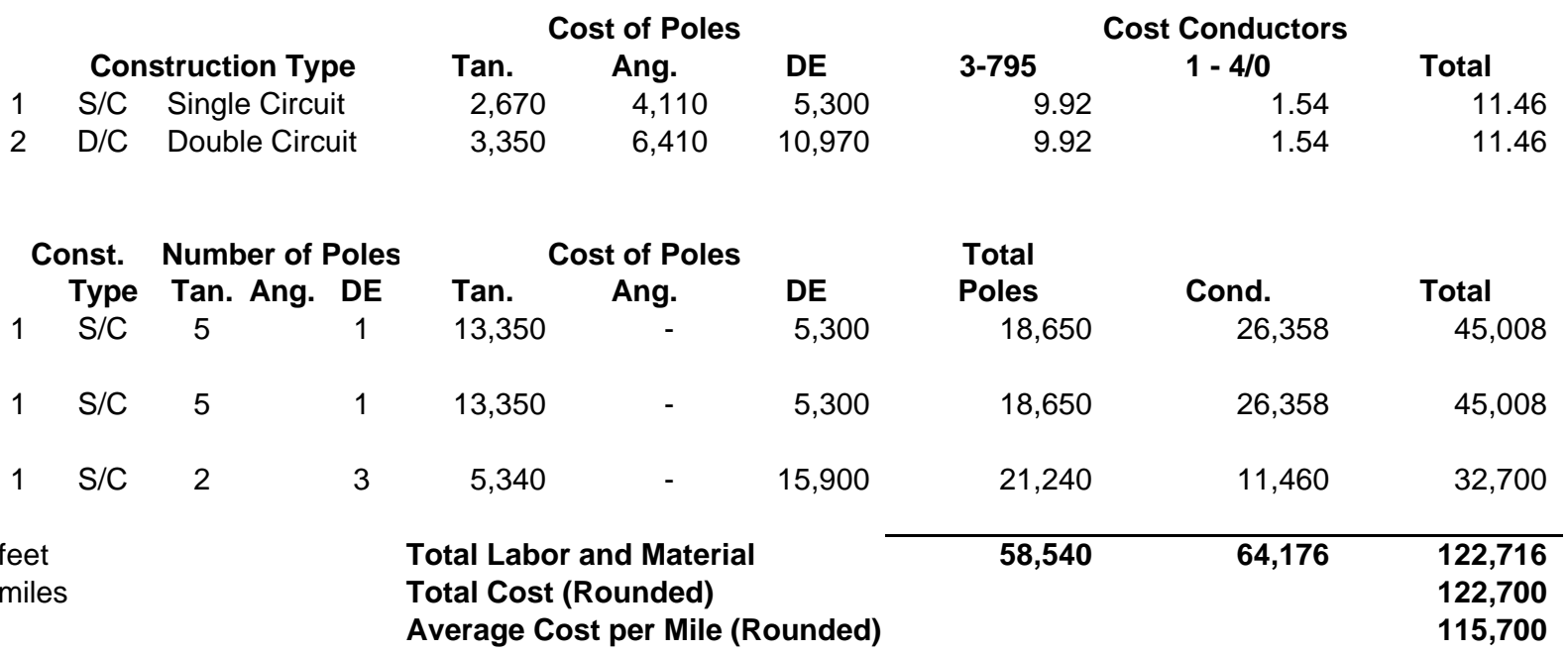


Table E6.2A

2500 kW - 25 kV Plan Overhead Line Cost Detail

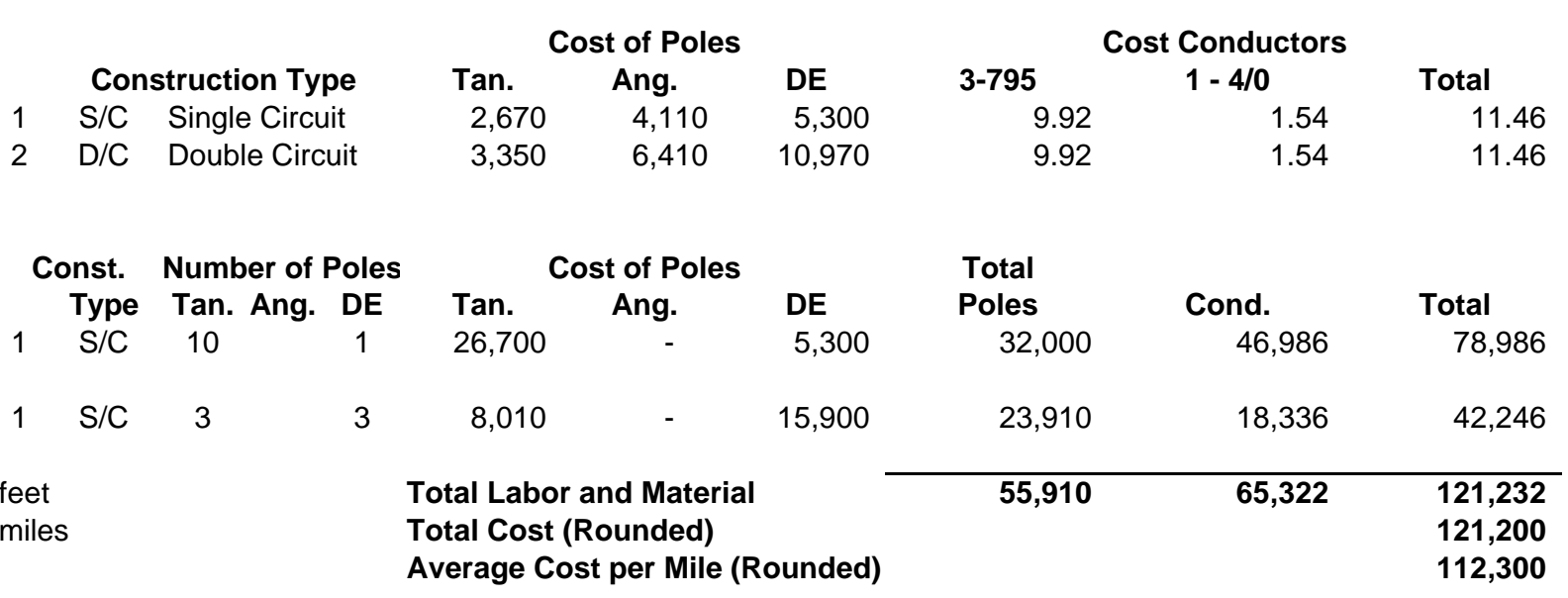


Table E6.2B

2500 kW - 35 kV Plan Overhead Line Cost Detail

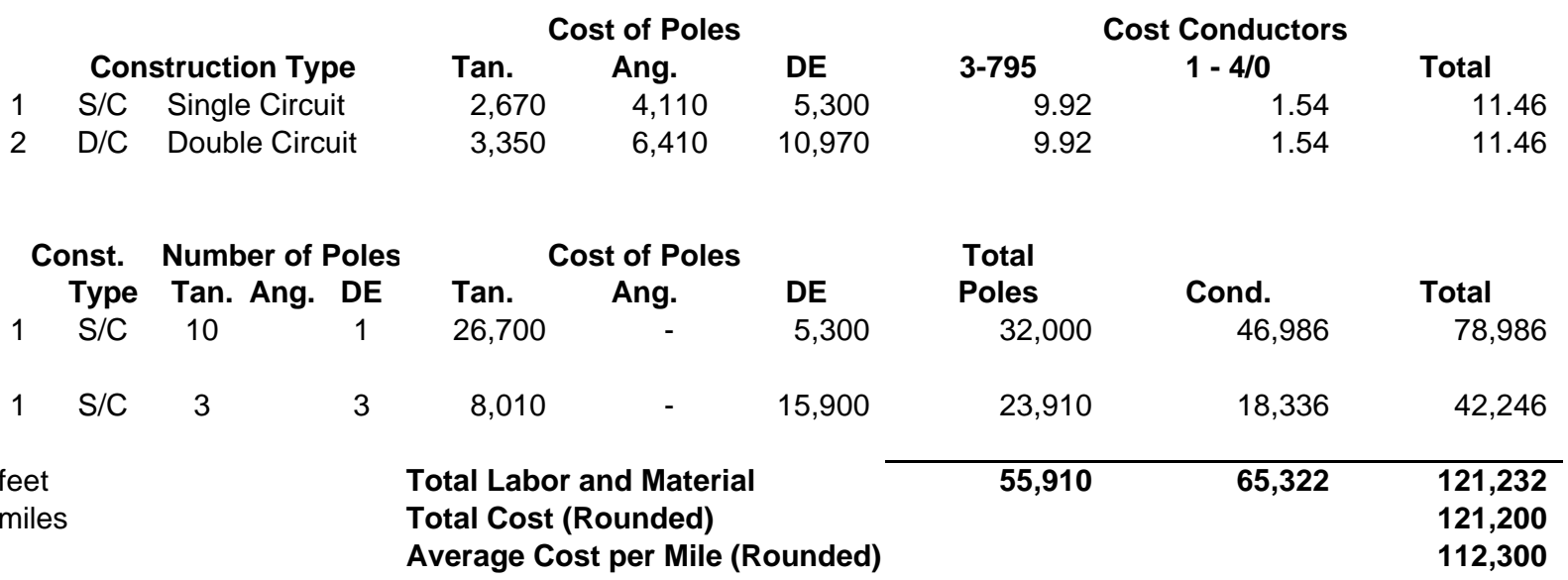


Table E6.3A

5000 kW - 25 kV Plan Overhead Line Cost Detail

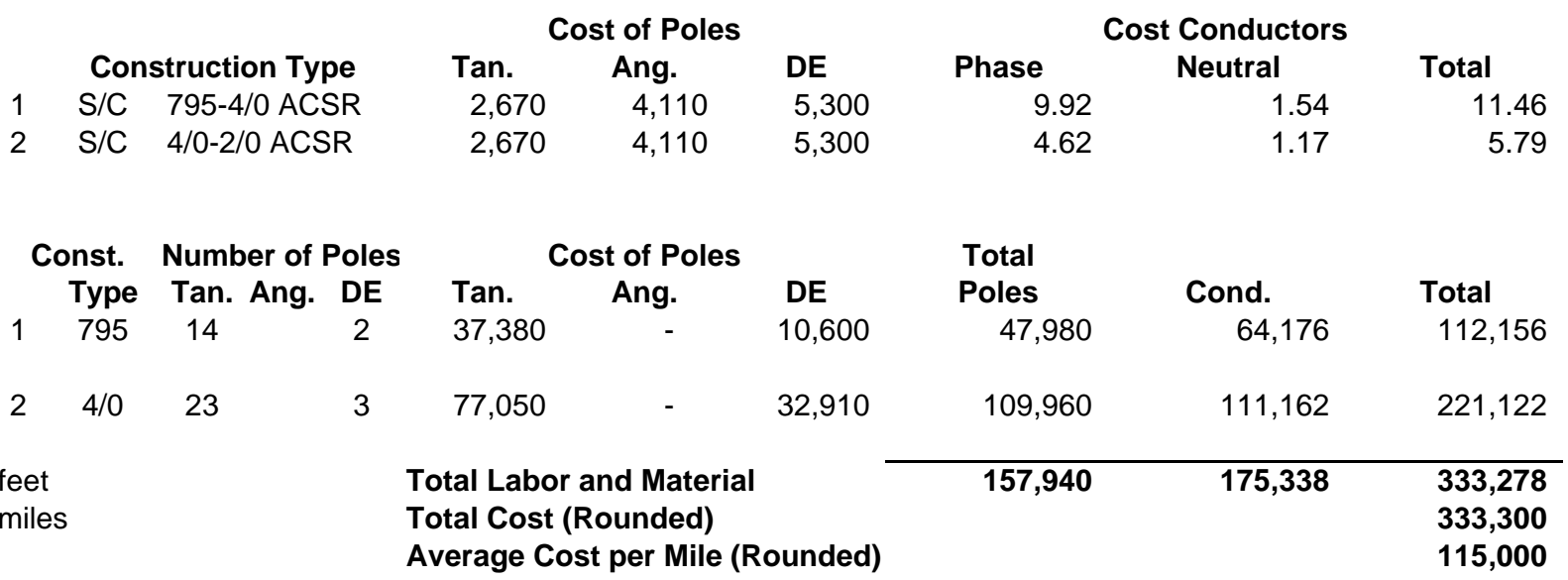


Table E6.3B

5000 kW - 35 kV Plan Overhead Line Cost Detail

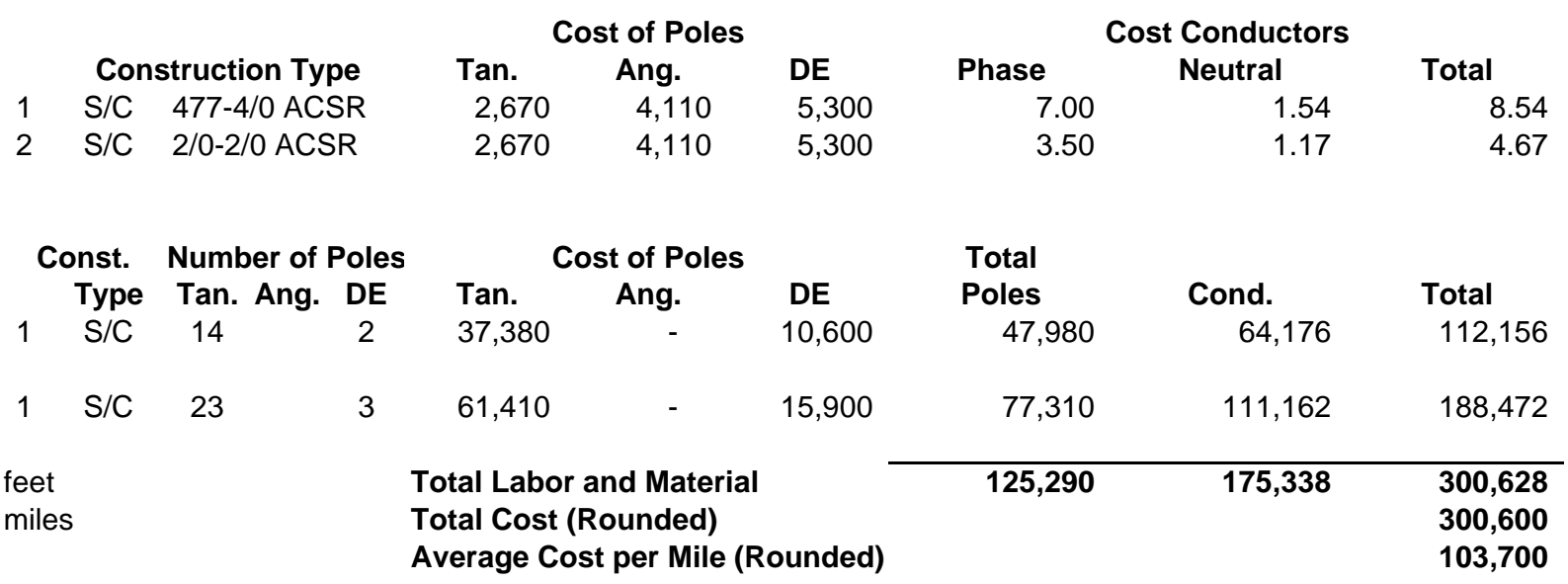


Table E6.4A

10000 kW - 25 kV Plan Overhead Line Cost Detail

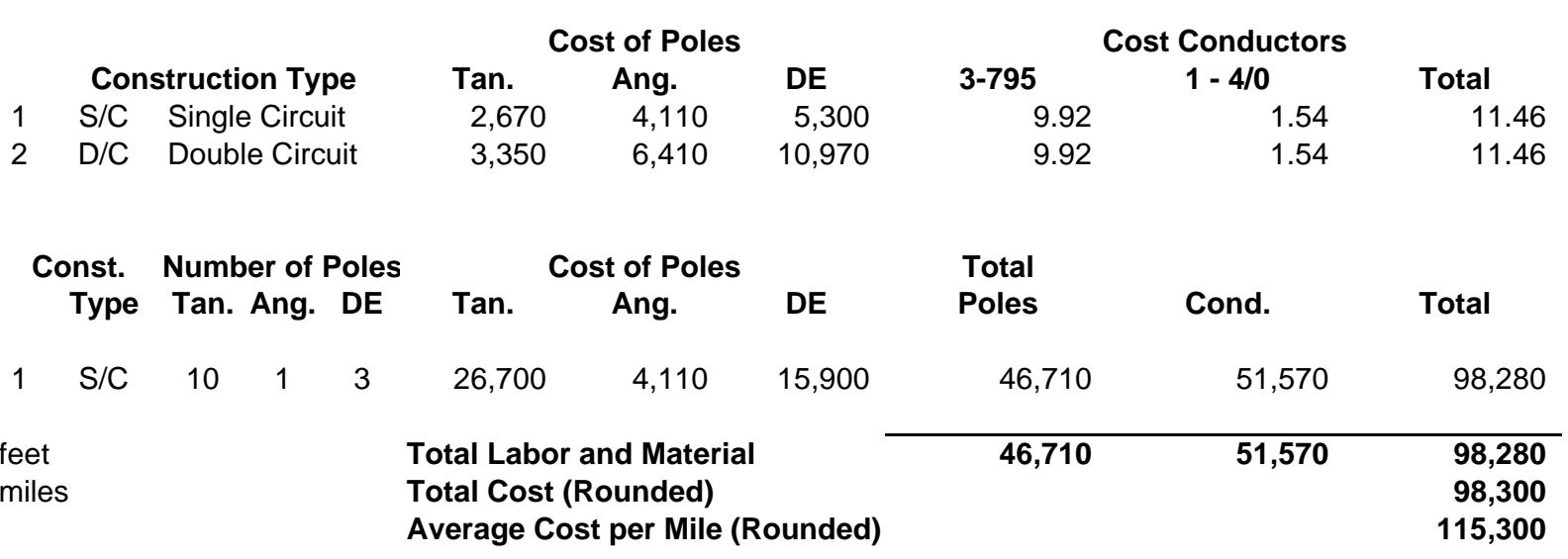


Table E6.4B

10000 kW - 35 kV Plan Overhead Line Cost Detail

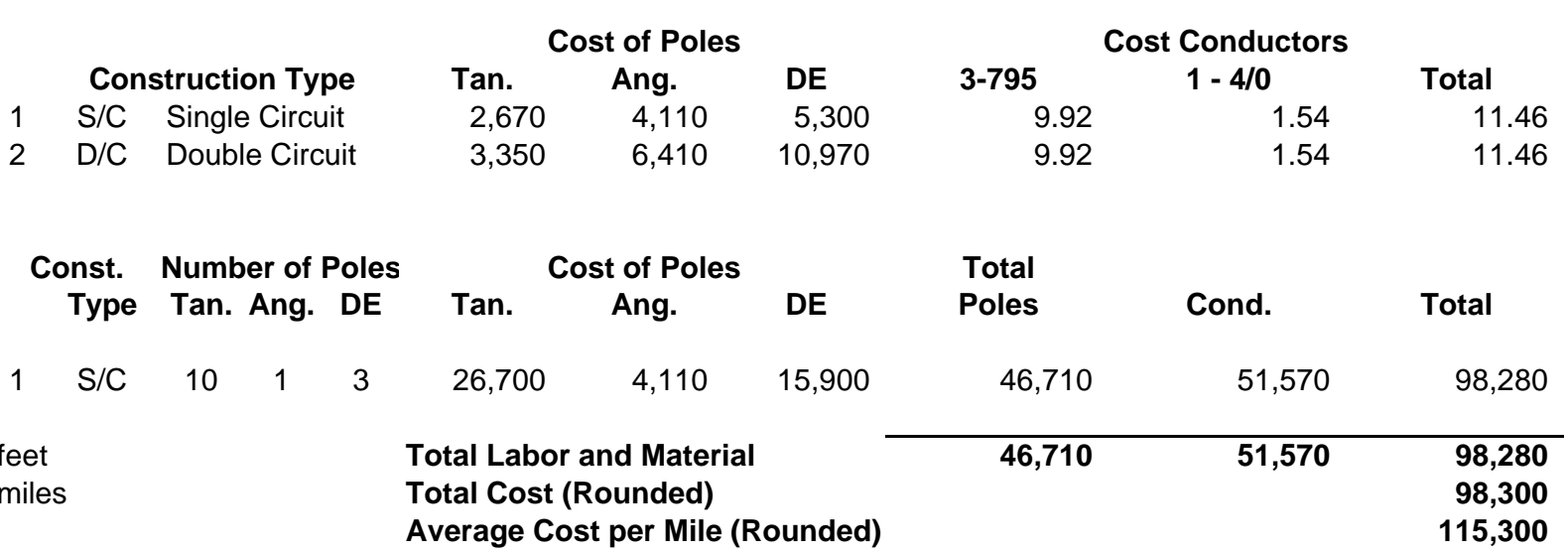


Table E7.1

New 15 kV PDS Substation Installed Adjacent Existing 115kV Line

\begin{tabular}{|c|c|c|c|c|}
\hline Description & Material & $\begin{array}{l}\text { Labor } \\
\text { Equipm }\end{array}$ & Total & $\begin{array}{c}\text { Total } \\
\text { Incl O\&P }\end{array}$ \\
\hline 115 kV Bus \& Foundations & 950,000 & 320,000 & $1,270,000$ & $1,422,400$ \\
\hline 30/40/50 PDS Substation, $115-13.8 \mathrm{kV}$ & 697,000 & 21,200 & 718,200 & 804,384 \\
\hline Foundation \& Oil Containment & 3,900 & 7,800 & 11,700 & 15,210 \\
\hline 13.8 Feeder Connection & 30,000 & 10,000 & 40,000 & 44,800 \\
\hline Power Factor Correction & 100,000 & 20,000 & 120,000 & 134,400 \\
\hline $115 \mathrm{kV}$ Metering & 150,000 & 20,000 & 170,000 & 190,400 \\
\hline $\begin{array}{r}\text { Total Substation } \\
\text { Total Substation (Rounded) }\end{array}$ & $1,930,900$ & 399,000 & $2,329,900$ & $\begin{array}{l}2,611,594 \\
2,612,000\end{array}$ \\
\hline
\end{tabular}

Land ( 3 acres at $\$ 5,000 /$ acre) All Plans

$\$ 15,000$

Table E7.2

New 25 kV PDS Substation Installed Adjacent Existing 115kV Line

\section{Description}

$115 \mathrm{kV}$ Bus \& Foundations

30/40/50 PDS Substation, 115-24.9 kV

Foundation \& Oil Containment

24.9 Feeder Connection

Power Factor Correction

115 kV Metering

Total Substation

Total Substation (Rounded)

\begin{tabular}{|c|c|c|c|}
\hline Material & $\begin{array}{l}\text { Labor } \\
\text { Equipm }\end{array}$ & Total & $\begin{array}{c}\text { Total } \\
\text { Incl O\&P }\end{array}$ \\
\hline 950,000 & 320,000 & $1,270,000$ & $1,422,400$ \\
\hline 642,000 & 21,200 & 663,200 & 742,784 \\
\hline 3,900 & 7,800 & 11,700 & 15,210 \\
\hline 15,000 & 5,000 & 20,000 & 22,400 \\
\hline 100,000 & 20,000 & 120,000 & 134,400 \\
\hline 150,000 & 20,000 & 170,000 & 190,400 \\
\hline $1,860,900$ & 394,000 & $2,254,900$ & $2,527,594$ \\
\hline
\end{tabular}

E7 - 1 
Table E7.3

New 35 kV PDS Substation Installed Adjacent Existing 115kV Line

\section{Description}

$115 \mathrm{kV}$ Bus \& Foundations

30/40/50 PDS Substation, 115-34.5 kV

Foundation \& Oil Containment

34.5 Feeder Connection

Power Factor Correction

$115 \mathrm{kV}$ Metering

Total Substation

Total Substation (Rounded)

\begin{tabular}{|c|c|c|c|}
\hline Material & $\begin{array}{l}\text { Labor } \\
\text { Equipm }\end{array}$ & Total & $\begin{array}{c}\text { Total } \\
\text { Incl O\&P }\end{array}$ \\
\hline 950,000 & 320,000 & $1,270,000$ & $1,422,400$ \\
\hline 662,000 & 21,200 & 683,200 & 765,184 \\
\hline 3,900 & 7,800 & 11,700 & 15,210 \\
\hline 15,000 & 5,000 & 20,000 & 22,400 \\
\hline 246,000 & 33,550 & 279,550 & 313,096 \\
\hline 150,000 & 20,000 & 170,000 & 190,400 \\
\hline \multirow[t]{2}{*}{$2,026,900$} & 407,550 & $2,434,450$ & $2,728,690$ \\
\hline & & & $2,729,000$ \\
\hline
\end{tabular}

E7 - 2 
Table E8.1

20' STONE SERVICE ROAD FOR 750 KW PLAN

\begin{tabular}{|c|c|c|c|c|c|c|}
\hline $\begin{array}{c}\text { Road } \\
\text { Section }\end{array}$ & $\begin{array}{c}\text { Road } \\
\text { Length } \\
\text { (feet) }\end{array}$ & $\begin{array}{l}\text { Road } \\
\text { Length } \\
\text { (miles) }\end{array}$ & $\begin{array}{c}\text { Unit } \\
\text { Cost } \\
\text { (per foot) }\end{array}$ & $\begin{array}{l}\text { Number } \\
\text { Stream } \\
\text { Crossings }\end{array}$ & $\begin{array}{l}\text { Unit } \\
\text { Cost } \\
\text { (Ea.) }\end{array}$ & $\begin{array}{c}\text { Road } \\
\text { Cost }\end{array}$ \\
\hline 1-Riser & 4147 & 0.8 & 50 & & 400 & 207,350 \\
\hline Riser-22 & 4147 & 0.8 & & & & 207,350 \\
\hline 23-Riser & 2639 & 0.5 & & & & 131,950 \\
\hline Riser-45 & 6032 & 1.1 & & \multicolumn{2}{|l|}{2} & 302,400 \\
\hline 46-Riser & 1131 & 0.2 & & & & 56,550 \\
\hline Riser-66 & 7540 & 1.4 & & \multicolumn{2}{|l|}{2} & 377,800 \\
\hline Totals & 25636 & 4.9 & & \multirow{2}{*}{\multicolumn{2}{|c|}{$\begin{array}{c}4 \\
\text { Total Rouno }\end{array}$}} & $1,283,400$ \\
\hline & & & & & & $1,290,000$ \\
\hline
\end{tabular}

Table E8.2

20' STONE SERVICE ROAD FOR 2,500 KW PLAN

\begin{tabular}{|c|c|c|c|c|c|c|}
\hline $\begin{array}{l}\text { Road } \\
\text { Section }\end{array}$ & $\begin{array}{l}\text { Road } \\
\text { Length } \\
\text { (feet) }\end{array}$ & $\begin{array}{l}\text { Road } \\
\text { Length } \\
\text { (miles) }\end{array}$ & $\begin{array}{c}\text { Unit } \\
\text { Cost } \\
\text { (per foot) }\end{array}$ & $\begin{array}{l}\text { Number } \\
\text { Stream } \\
\text { Crossings }\end{array}$ & $\begin{array}{l}\text { Unit } \\
\text { Cost } \\
\text { (Ea.) }\end{array}$ & $\begin{array}{l}\text { Road } \\
\text { Cost }\end{array}$ \\
\hline 1-Riser & 3852 & 0.7 & 50 & & 400 & 192,600 \\
\hline Riser-11 & 3210 & 0.6 & & & & 160,500 \\
\hline Riser-20 & 5778 & 1.1 & & 1 & & 289,300 \\
\hline Totals & 12840 & 2.4 & & 1 & & 642,400 \\
\hline
\end{tabular}

Table E8.3

20' STONE SERVICE ROAD FOR 5,000 KW PLAN

\begin{tabular}{lrrrrrr}
$\begin{array}{c}\text { Road } \\
\text { Section }\end{array}$ & $\begin{array}{c}\text { Road } \\
\text { Length } \\
\text { (feet) }\end{array}$ & $\begin{array}{c}\text { Road } \\
\text { Length } \\
\text { (miles) }\end{array}$ & $\begin{array}{c}\text { Unit } \\
\text { Cost } \\
\text { (per foot) }\end{array}$ & $\begin{array}{c}\text { Number } \\
\text { Stream } \\
\text { Crossings }\end{array}$ & $\begin{array}{c}\text { Unit } \\
\text { Cost } \\
\text { (Ea.) }\end{array}$ & $\begin{array}{c}\text { Road } \\
\text { Cost }\end{array}$ \\
1-Riser & 2718 & 0.5 & 50 & 400 & 135,900 \\
Riser-7 & 3624 & 0.7 & & & & 181,200 \\
Riser-10 & 2718 & 0.5 & & & 135,900 \\
\hline Totals & 9060 & 1.7 & & 0 & 453,000 \\
& & & & Total Rounded & 460,000
\end{tabular}

Table E8.4

20' STONE SERVICE ROAD FOR 10,000 KW PLAN

\begin{tabular}{|c|c|c|c|c|c|c|}
\hline $\begin{array}{c}\text { Road } \\
\text { Section }\end{array}$ & $\begin{array}{l}\text { Road } \\
\text { Length } \\
\text { (feet) }\end{array}$ & $\begin{array}{l}\text { Road } \\
\text { Length } \\
\text { (miles) }\end{array}$ & $\begin{array}{c}\text { Unit } \\
\text { Cost } \\
\text { (per foot) }\end{array}$ & $\begin{array}{c}\text { Number } \\
\text { Stream } \\
\text { Crossings }\end{array}$ & $\begin{array}{l}\text { Unit } \\
\text { Cost } \\
\text { (Ea.) }\end{array}$ & $\begin{array}{l}\text { Road } \\
\text { Cost }\end{array}$ \\
\hline Riser-1 & 2566 & 0.5 & 50 & & 400 & 128,300 \\
\hline Riser-3 & 800 & 0.2 & & & & 40,000 \\
\hline Riser-5 & 2783 & 0.5 & & & & 139,150 \\
\hline \multirow[t]{2}{*}{ Totals } & 6149 & 1.2 & & 0 & & 307,450 \\
\hline & & & & Total Round & & 310,000 \\
\hline
\end{tabular}


Table E8.1a

26' Paved SERVICE ROAD FOR 750 KW PLAN

\begin{tabular}{|c|c|c|c|c|c|c|}
\hline $\begin{array}{l}\text { Road } \\
\text { Section }\end{array}$ & $\begin{array}{c}\text { Road } \\
\text { Length } \\
\text { (feet) }\end{array}$ & $\begin{array}{c}\text { Road } \\
\text { Length } \\
\text { (miles) }\end{array}$ & $\begin{array}{c}\text { Unit } \\
\text { Cost } \\
\text { (per foot) }\end{array}$ & $\begin{array}{c}\text { Number } \\
\text { Stream } \\
\text { Crossings }\end{array}$ & $\begin{array}{l}\text { Unit } \\
\text { Cost } \\
\text { (Ea.) }\end{array}$ & $\begin{array}{c}\text { Road } \\
\text { Cost }\end{array}$ \\
\hline 1-Riser & 4147 & 0.8 & 90 & & 400 & 373,230 \\
\hline Riser-22 & 4147 & 0.8 & & & & 373,230 \\
\hline 23-Riser & 2639 & 0.5 & & & & 237,510 \\
\hline Riser-45 & 6032 & 1.1 & & 2 & & 543,680 \\
\hline 46-Riser & 1131 & 0.2 & & & & 101,790 \\
\hline Riser-66 & 7540 & 1.4 & & 2 & & 679,400 \\
\hline \multirow[t]{2}{*}{ Totals } & 25636 & 4.9 & & 4 & & $2,308,840$ \\
\hline & 25600 & & & Total Rounde & & $2,310,000$ \\
\hline
\end{tabular}

Table E8.2a

26' Paved SERVICE ROAD FOR 2,500 KW PLAN

\begin{tabular}{lrrrrrr}
$\begin{array}{c}\text { Road } \\
\text { Section }\end{array}$ & $\begin{array}{c}\text { Road } \\
\text { Length } \\
\text { (feet) }\end{array}$ & $\begin{array}{c}\text { Road } \\
\text { Length } \\
\text { (miles) }\end{array}$ & $\begin{array}{c}\text { Unit } \\
\text { Cost } \\
\text { (per foot) }\end{array}$ & $\begin{array}{c}\text { Number } \\
\text { Stream } \\
\text { Crossings }\end{array}$ & $\begin{array}{l}\text { Unit } \\
\text { Cost } \\
\text { (Ea.) }\end{array}$ & $\begin{array}{c}\text { Road } \\
\text { Cost }\end{array}$ \\
1-Riser & 3852 & 0.7 & 90 & 400 & 346,680 \\
Riser-11 & 3210 & 0.6 & & & & 288,900 \\
Riser-20 & 5778 & 1.1 & 1 & 1 & 520,420 \\
\hline Totals & 12840 & 2.4 & Total Rounded & $1,156,000$ \\
& 12800 & & & & & $1,156,000$
\end{tabular}

Table E8.3a

32' Paved SERVICE ROAD FOR 5,000 KW PLAN

\begin{tabular}{lrrrrrr}
$\begin{array}{c}\text { Road } \\
\text { Section }\end{array}$ & $\begin{array}{c}\text { Road } \\
\text { Length } \\
\text { (feet) }\end{array}$ & $\begin{array}{c}\text { Road } \\
\text { Length } \\
\text { (miles) }\end{array}$ & $\begin{array}{c}\text { Unit } \\
\text { Cost } \\
\text { (per foot) }\end{array}$ & $\begin{array}{c}\text { Number } \\
\text { Stream } \\
\text { Crossings }\end{array}$ & $\begin{array}{l}\text { Unit } \\
\text { Cost } \\
\text { (Ea.) }\end{array}$ & $\begin{array}{r}\text { Road } \\
\text { Cost }\end{array}$ \\
1-Riser & 2718 & 0.5 & 110 & 400 & 298,980 \\
Riser-7 & 3624 & 0.7 & & & & 398,640 \\
Riser-10 & 2718 & 0.5 & & 0 & 298,980 \\
\hline Totals & 9060 & 1.7 & & Total Rounded & $1,000,000$
\end{tabular}

Table E8.4a

32' Paved SERVICE ROAD FOR 10,000 KW PLAN

\begin{tabular}{lrrrrrr}
$\begin{array}{c}\text { Road } \\
\text { Section }\end{array}$ & $\begin{array}{c}\text { Road } \\
\text { Length } \\
\text { (feet) }\end{array}$ & $\begin{array}{c}\text { Road } \\
\text { Length } \\
\text { (miles) }\end{array}$ & $\begin{array}{c}\text { Unit } \\
\text { Cost } \\
\text { (per foot) }\end{array}$ & $\begin{array}{c}\text { Number } \\
\text { Stream } \\
\text { Crossings }\end{array}$ & $\begin{array}{l}\text { Unit } \\
\text { Cost } \\
\text { (Ea.) }\end{array}$ & $\begin{array}{r}\text { Road } \\
\text { Cost }\end{array}$ \\
Riser-1 & 2566 & 0.5 & 110 & 400 & 282,260 \\
Riser-3 & 800 & 0.2 & & & & 88,000 \\
Riser-5 & 2783 & 0.5 & & 0 & 306,130 \\
\hline Totals & 6149 & 1.2 & Total Rounded & 676,390 \\
& 6150 & & & & 680,000
\end{tabular}


Table E8.1b

30' Paved SERVICE ROAD FOR 750 KW PLAN

\begin{tabular}{|c|c|c|c|c|c|c|}
\hline $\begin{array}{l}\text { Road } \\
\text { Section }\end{array}$ & $\begin{array}{c}\text { Road } \\
\text { Length } \\
\text { (feet) }\end{array}$ & $\begin{array}{l}\text { Road } \\
\text { Length } \\
\text { (miles) }\end{array}$ & $\begin{array}{c}\text { Unit } \\
\text { Cost } \\
\text { (per foot) }\end{array}$ & $\begin{array}{c}\text { Number } \\
\text { Stream } \\
\text { Crossings }\end{array}$ & $\begin{array}{l}\text { Unit } \\
\text { Cost } \\
\text { (Ea.) }\end{array}$ & $\begin{array}{l}\text { Road } \\
\text { Cost }\end{array}$ \\
\hline 1-Riser & 4147 & 0.8 & 110 & & 400 & 456,170 \\
\hline Riser-22 & 4147 & 0.8 & & & & 456,170 \\
\hline 23-Riser & 2639 & 0.5 & & & & 290,290 \\
\hline Riser-45 & 6032 & 1.1 & & 2 & & 664,320 \\
\hline 46-Riser & 1131 & 0.2 & & & & 124,410 \\
\hline Riser-66 & 7540 & 1.4 & & 2 & & 830,200 \\
\hline \multirow[t]{2}{*}{ Totals } & 25636 & 4.9 & & 4 & & $2,821,560$ \\
\hline & & & & Total Roun & & $2,830,000$ \\
\hline
\end{tabular}

Table E8.2b

30' Paved SERVICE ROAD FOR 2,500 KW PLAN

\begin{tabular}{lrrrrrr}
$\begin{array}{c}\text { Road } \\
\text { Section }\end{array}$ & $\begin{array}{c}\text { Road } \\
\text { Length } \\
\text { (feet) }\end{array}$ & $\begin{array}{c}\text { Road } \\
\text { Length } \\
\text { (miles) }\end{array}$ & $\begin{array}{c}\text { Unit } \\
\text { Cost } \\
\text { (per foot) }\end{array}$ & $\begin{array}{c}\text { Number } \\
\text { Stream } \\
\text { Crossings }\end{array}$ & $\begin{array}{l}\text { Unit } \\
\text { Cost } \\
\text { (Ea.) }\end{array}$ & $\begin{array}{c}\text { Road } \\
\text { Cost }\end{array}$ \\
1-Riser & 3852 & 0.7 & 110 & 400 & 423,720 \\
Riser-11 & 3210 & 0.6 & & 1 & & 353,100 \\
Riser-20 & 5778 & 1.1 & & 1 & 635,980 \\
\hline Totals & 12840 & 2.4 & Total Rounded & $1,412,800$ \\
& & & & & & $1,413,000$
\end{tabular}

Table E8.3b

40' Paved SERVICE ROAD FOR 5,000 KW PLAN

\begin{tabular}{lrrrrrr}
$\begin{array}{c}\text { Road } \\
\text { Section }\end{array}$ & $\begin{array}{c}\text { Road } \\
\text { Length } \\
\text { (feet) }\end{array}$ & $\begin{array}{c}\text { Road } \\
\text { Length } \\
\text { (miles) }\end{array}$ & $\begin{array}{c}\text { Unit } \\
\text { Cost } \\
\text { (per foot) }\end{array}$ & $\begin{array}{c}\text { Number } \\
\text { Stream } \\
\text { Crossings }\end{array}$ & $\begin{array}{l}\text { Unit } \\
\text { Cost } \\
\text { (Ea.) }\end{array}$ & $\begin{array}{c}\text { Road } \\
\text { Cost }\end{array}$ \\
1-Riser & 2718 & 0.5 & 140 & 400 & 380,520 \\
Riser-7 & 3624 & 0.7 & & & & 507,360 \\
Riser-10 & 2718 & 0.5 & & 0 & 380,520 \\
\hline Totals & 9060 & 1.7 & Total Rounded & $1,268,400$ \\
& & & & & & $1,270,000$
\end{tabular}

Table E8.4b

40' Paved SERVICE ROAD FOR 10,000 KW PLAN

\begin{tabular}{lrrrrrr}
$\begin{array}{c}\text { Road } \\
\text { Section }\end{array}$ & $\begin{array}{c}\text { Road } \\
\text { Length } \\
\text { (feet) }\end{array}$ & $\begin{array}{c}\text { Road } \\
\text { Length } \\
\text { (miles) }\end{array}$ & $\begin{array}{c}\text { Unit } \\
\text { Cost } \\
\text { (per foot) }\end{array}$ & $\begin{array}{c}\text { Number } \\
\text { Stream } \\
\text { Crossings }\end{array}$ & $\begin{array}{l}\text { Unit } \\
\text { Cost } \\
\text { (Ea.) }\end{array}$ & $\begin{array}{c}\text { Road } \\
\text { Cost }\end{array}$ \\
Riser-1 & 2566 & 0.5 & 140 & 400 & 359,240 \\
Riser-3 & 800 & 0.2 & & & & 112,000 \\
Riser-5 & 2783 & 0.5 & & 0 & 389,620 \\
\hline Totals & 6149 & 1.2 & Total Rounded & 860,860 \\
& & & & & & 870,000
\end{tabular}




\section{Table E9 \\ Maintenance Building All Plans}

\author{
Description \\ 2,400 Square Foot Service Building \\ Water \\ Septic \\ Driveway/Parking \\ Security Fence \\ Gate
}

Land (Acres)

Site Clearing

Site Preparation

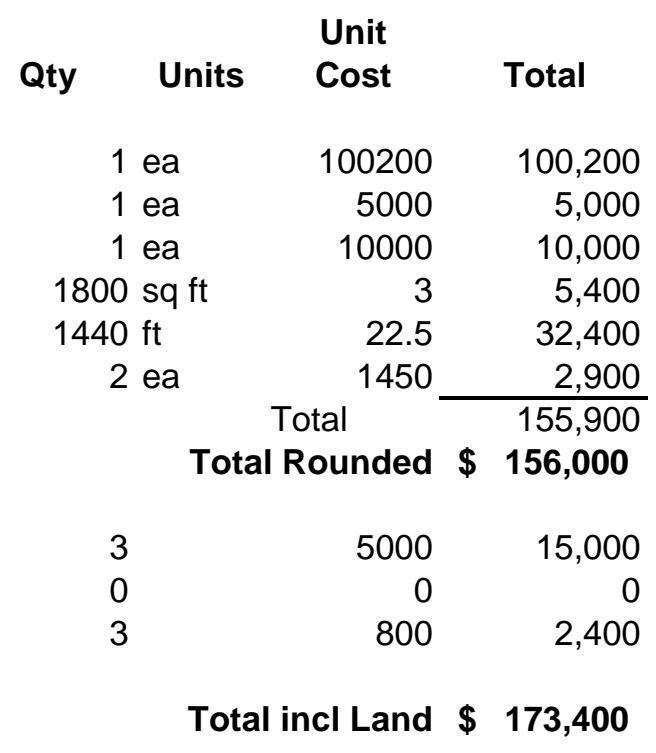




\section{Table E10}

\section{Communications Cost Detail}

Item Description

1 Scada Master Station

2 Scada RTU with Modem

3 Underground Fiber Optic

Installed in Open Utility Trench

4 Overhead Fiber Optic

\begin{tabular}{cr} 
& \multicolumn{1}{c}{ Unit } \\
Unit & Cost \\
Ea. & 66,700 \\
Ea. & 8,050 \\
Ft. & 4.56 \\
Ft. & 7.09
\end{tabular}

Table E10.1

750 kW - Communications Cost Detail

\begin{tabular}{|c|c|c|c|c|}
\hline & Unit & Total & & \\
\hline Qty & Cost & Cost & & \\
\hline 1 & 66,700 & 66,700 & & \\
\hline \multirow[t]{2}{*}{73} & 8,050 & 587,650 & & Rounded \\
\hline & \multicolumn{2}{|c|}{ Subtotal SCADA } & 654,350 & 654,000 \\
\hline \multirow{4}{*}{$\begin{array}{r}28,200 \\
5,600\end{array}$} & 4.56 & 128,592 & & \\
\hline & 7.09 & 39,704 & & \\
\hline & \multirow{2}{*}{\multicolumn{2}{|c|}{$\begin{array}{r}\text { Subtotal Fiber Optics } \\
\text { Total Cost }\end{array}$}} & 168,296 & 168,000 \\
\hline & & & 822,646 & 823,000 \\
\hline
\end{tabular}

Table E10.2

2500 kW - Communications Cost Detail

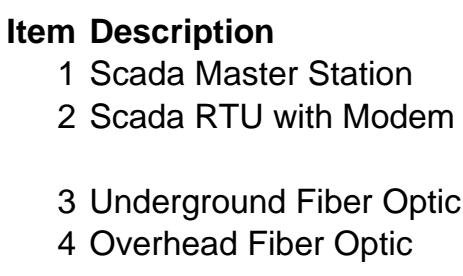

Item Description

1 Scada Master Station

3 Underground Fiber Optic

4 Overhead Fiber Optic

\begin{tabular}{|c|c|c|c|c|}
\hline 0 & Unit & Total & & \\
\hline 1 & 66,700 & 66,700 & & \\
\hline \multirow[t]{2}{*}{25} & 8,050 & 201,250 & & Rounded \\
\hline & \multicolumn{2}{|c|}{ Subtotal SCADA } & 267,950 & 268,000 \\
\hline 13,900 & 4.56 & 63,384 & & \\
\hline \multirow[t]{3}{*}{5,700} & 7.09 & 40,413 & & \\
\hline & \multicolumn{2}{|c|}{ Subtotal Fiber Optics } & 103,797 & 104,000 \\
\hline & \multicolumn{2}{|c|}{ Total Cost } & 371,747 & 372,000 \\
\hline
\end{tabular}


Table E10.3

5000 kW - Communications Cost Detail

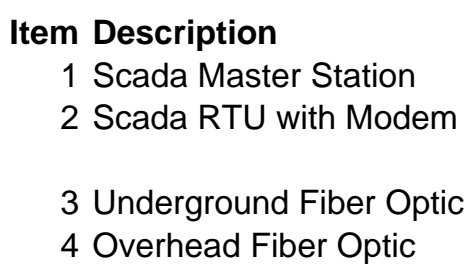

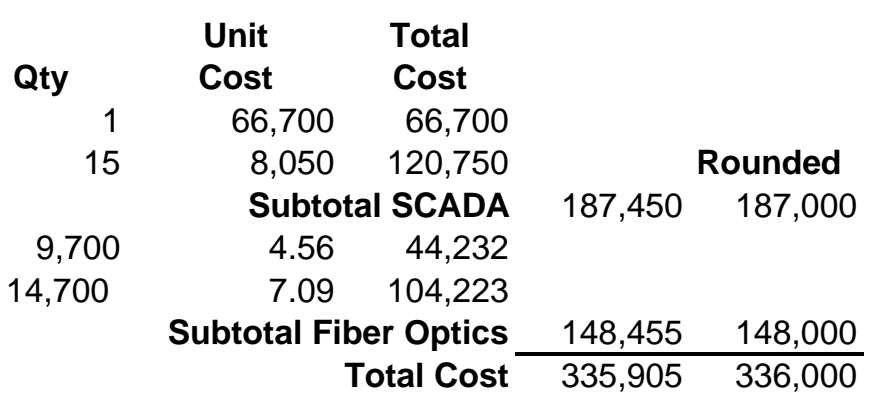

Table E10.4

\section{0 kW - Communications Cost Detail}

\begin{tabular}{|c|c|c|c|c|}
\hline & Unit & Total & & \\
\hline Qty & Cost & Cost & & \\
\hline 1 & 66,700 & 66,700 & & \\
\hline \multirow[t]{2}{*}{10} & 8,050 & 80,500 & & Rounded \\
\hline & \multicolumn{2}{|c|}{ Subtotal SCADA } & 147,200 & 147,000 \\
\hline 6,600 & 4.56 & 30,096 & & \\
\hline \multirow[t]{2}{*}{4,500} & 7.09 & 31,905 & & \\
\hline & \multicolumn{2}{|c|}{$\begin{array}{r}\text { Subtotal Fiber Optics } \\
\text { Total Cost }\end{array}$} & $\frac{62,001}{209201}$ & $\begin{array}{r}62,000 \\
209000\end{array}$ \\
\hline
\end{tabular}


Table E11

\section{Crane Pad Cost Detail}

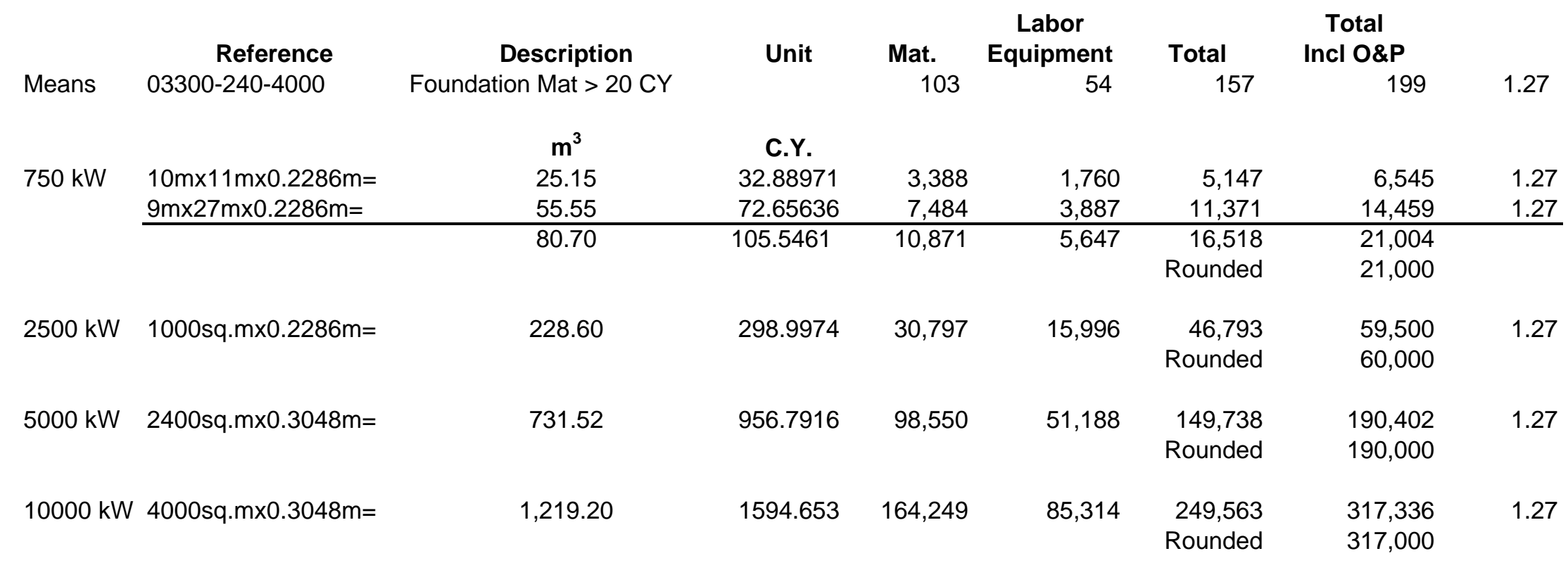




\section{Table E12}

\section{Vendor Quote for the Major Electrical Equipment}

\begin{tabular}{|c|c|c|c|c|c|}
\hline Item & \multicolumn{2}{|c|}{ Description } & Manufacturer & Quote & Uni \\
\hline PDS Substation & & $15 \mathrm{kV}$ & Waukesha & $\$ 697,000$ & Ea. \\
\hline PDS Substation & & $25 \mathrm{kV}$ & Waukesha & $\$ 642,000$ & Ea. \\
\hline PDS Substation & & $35 \mathrm{kV}$ & Waukesha & $\$ 662,000$ & Ea. \\
\hline Power Factor Correction & IntelliVAr & & PQS & $\$ 100,000$ & Ea. \\
\hline Padmount Transformer & $1000 \mathrm{kVA}$ & $15 \mathrm{kV}$ & Cooper & $\$ 11,375$ & Ea. \\
\hline Padmount Transformer & $1000 \mathrm{kVA}$ & $25 \mathrm{kV}$ & Cooper & $\$ 11,400$ & Ea. \\
\hline Padmount Transformer & $3000 \mathrm{kVA}$ & $25 \mathrm{kV}$ & Cooper & $\$ 23,500$ & Ea. \\
\hline Padmount Transformer & $3000 \mathrm{kVA}$ & $35 \mathrm{kV}$ & Cooper & $\$ 25,000$ & Ea. \\
\hline Padmount Transformer & 7500 kVA & $25 \mathrm{kV}$ & Cooper & $\$ 81,800$ & Ea. \\
\hline Padmount Transformer & $7500 \mathrm{kVA}$ & $35 \mathrm{kV}$ & Cooper & $\$ 83,200$ & Ea. \\
\hline Substation Transformer & $10,000 \mathrm{kVA}$ & $25 \mathrm{kV}$ & Cooper & $\$ 90,000$ & Ea. \\
\hline Substation Transformer & $10,000 \mathrm{kVA}$ & $35 \mathrm{kV}$ & Cooper & $\$ 92,000$ & Ea. \\
\hline Switchgear & & $25 \mathrm{kV}$ & Cooper & $\$ 17,620$ & Ea. \\
\hline Switchgear & & $35 \mathrm{kV}$ & Cooper & $\$ 24,380$ & Ea. \\
\hline Recloser & & $15 \mathrm{kV}$ & Cooper & $\$ 15,750$ & Ea. \\
\hline Recloser & & $25 \mathrm{kV}$ & Cooper & $\$ 16,700$ & Ea. \\
\hline Recloser & & $35 \mathrm{kV}$ & Cooper & $\$ 18,400$ & Ea. \\
\hline Underground Cable & $1 / 0$ & $15 \mathrm{kV}$ & Okonite & $\$ 1.118$ & Ft. \\
\hline Underground Cable & $4 / 0$ & $15 \mathrm{kV}$ & Okonite & $\$ 2.003$ & Ft. \\
\hline Underground Cable & 500 & $15 \mathrm{kV}$ & Okonite & $\$ 3.646$ & Ft. \\
\hline Underground Cable & 750 & $15 \mathrm{kV}$ & Okonite & $\$ 4.991$ & Ft. \\
\hline Underground Cable & $1 / 0$ & $25 \mathrm{kV}$ & Okonite & $\$ 1.395$ & Ft. \\
\hline Underground Cable & $4 / 0$ & $25 \mathrm{kV}$ & Okonite & $\$ 1.542$ & Ft. \\
\hline Underground Cable & 500 & $25 \mathrm{kV}$ & Okonite & $\$ 1.932$ & Ft. \\
\hline Underground Cable & $1 / 0$ & $35 \mathrm{kV}$ & Okonite & $\$ 2.134$ & Ft. \\
\hline Underground Cable & $4 / 0$ & $35 \mathrm{kV}$ & Okonite & $\$ 1.812$ & $\mathrm{Ft}$ \\
\hline Underground Cable & 500 & $35 \mathrm{kV}$ & Okonite & $\$ 2.174$ & $\mathrm{Ct}$ \\
\hline Overhead ACSR & 795 & & Southwire & $\$ 1.21$ & the \\
\hline Overhead ACSR & 477 & & Southwire & $\$ 1.38$ & \\
\hline Overhead ACSR & $4 / 0$ & & Southwire & $\$ 1.16$ & 10 \\
\hline Overhead ACSR & $2 / 0$ & & Southwire & $\$ 1.17$ & $\mathrm{lb}$ \\
\hline SCADA Master Station & Model 9200 & & Ilex & $\$ 55,000$ & \\
\hline SCADA RTU & Model 9300 & & Ilex & $\$ 5,000$ & \\
\hline
\end{tabular}




\section{REPORT DOCUMENTATION PAGE}

Form Approved

OMB NO. 0704-0188

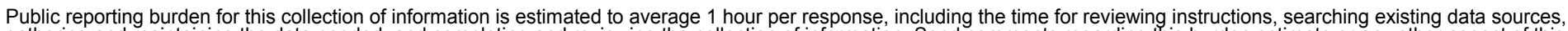

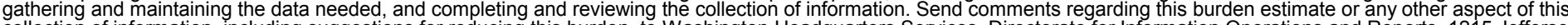

Davis Highway, Suite 1204, Arlington, VA 22202-4302, and to the Office of Management and Budget, Paperwork Reduction Project (0704-0188), Washington, DC 20503.

\begin{tabular}{|l|l|l} 
1. AGENCY USE ONLY (Leave blank) & $\begin{array}{c}\text { 2. REPORT DATE } \\
\text { July } 2001\end{array}$ & $\begin{array}{l}\text { 3. REPORT TYPE AND DATES COVERED } \\
\text { Subcontract report }\end{array}$
\end{tabular}

4. TITLE AND SUBTITLE

WindPACT Turbine Design Scaling Studies: Technical Area 4-Balance-of-Station Cost

5. FUNDING NUMBERS

C: YAM-10-30203-02

6. $\mathrm{AUTHOR}(\mathrm{S})$

D.A. Shafer, K.R. Strawmyer, R.M. Conley, J.H. Guidinger, D.C. Wilkie, T.F. Zellman

TA: WER1-1110

7. PERFORMING ORGANIZATION NAME(S) AND ADDRESS(ES)

Commonwealth Associates Inc.

P.O. Box 1124, Jackson, MI 49204-1124 REPORT NUMBER

9. SPONSORISIMONITORNGAGENCYNAME(S)ANDADDRES(ES)

9. SPONSORING/MONITORING AGENCY NAME(S) AND ADDRESS(ES)

National Renewable Energy Laboratory

1617 Cole Blvd.

Golden, CO 80401-3393

10. SPONSORING/MONITORING AGENCY REPORT NUMBER

NREL/SR-500-29950

11. SUPPLEMENTARY NOTES

NREL Technical Monitor: Alan Laxson

12a. DISTRIBUTION/AVAILABILITY STATEMENT

National Technical Information Service

12b. DISTRIBUTION CODE

U.S. Department of Commerce

5285 Port Royal Road

Springfield, VA 22161

13. ABSTRACT (Maximum 200 words)

DOE's Wind Partnerships for Advanced Component Technologies (WindPACT) program explores the most advanced wind-generating technologies for improving reliability and decreasing energy costs. The first step in the WindPact program is a scaling study to bound the optimum sizes for wind turbines, to define size limits for certain technologies, and to scale new technologies. The program is divided into four projects: Composite Blades for 80-120-meter Rotors; Turbine, Rotor, and Blade Logistics; Self-Erecting Tower and Nacelle Feasibility; and Balance-of-Station Cost. This report discusses balance-of-station costs, which includes the electrical power collector system, wind turbine foundations, communications and controls, meteorological equipment, access roadways, crane pads, and the maintenance building. The report is based on a conceptual 50-megawatt (MW) wind farm site near Mission, South Dakota. Cost comparisons are provided for four sizes of wind turbines: 750 kilowatt (kW), $2.5 \mathrm{MW}, 5.0 \mathrm{MW}$, and 10.0 MW.

14. SUBJECT TERMS

Wind energy; aerodynamics experiment; NASA Ames; wind tunnel

15. NUMBER OF PAGES

16. PRICE CODE

17. SECURITY CLASSIFICATION OF REPORT Unclassified
18. SECURITY CLASSIFICATION OF THIS PAGE Unclassified
19. SECURITY CLASSIFICATION OF ABSTRACT Unclassified
20. LIMITATION OF ABSTRACT

UL 